\title{
Measurement of $Z^{0} \gamma$ Production in $p \bar{p}$ Collisions at $\sqrt{s}=1.96 \mathrm{TeV}$
}

\author{
Helen Sarah Hayward
}

March 2005

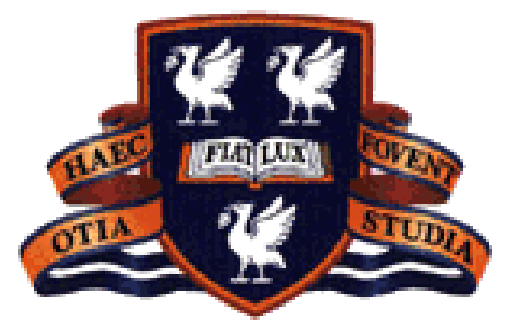

University of Liverpool

Department of Physics

Thesis submitted in accordance with the requirements of the University of Liverpool for the degree of Doctor in Philosophy 


\section{Abstract}

The cross-section of $p \bar{p} \rightarrow Z^{0} \gamma+X$ production at $\sqrt{s}=1.96 \mathrm{TeV}$ using the CDF II detector at the Fermilab Tevatron is measured using $202 \mathrm{pb}^{-1}$ of data from March 2002 to September 2003, collected with high $P_{T}$ lepton triggers. The number of events observed and their kinematic distributions are compared to the NLO Standard Model prediction in both the $Z^{0} \rightarrow e^{+} e^{-}$ and $Z^{0} \rightarrow \mu^{+} \mu^{-}$channels. For a minimum lepton-photon separation of $\Delta R_{l \gamma}>0.7, E_{T}^{\gamma}>7 \mathrm{GeV}$ and a dilepton invariant mass $m(l, l)>40 \mathrm{GeV} / \mathrm{c}^{2}$, the cross-section times branching ratio is measured to be $4.6 \pm 0.5$ (stat) \pm 0.2 (sys) \pm 0.3 (lumi) pb compared to the NLO Standard Model prediction of $4.5 \pm 0.3(\mathrm{th}) \mathrm{pb}$. 


\section{Acknowledgments}

I am immensely grateful to my supervisor, Beate Heinemann for her support, advice and knowledge. Thanks also to Al Goshaw, Mike Kirby and Naho Tanimoto for making me feel extremely welcome as the newcomer into the Vgamma working group at CDF. Their support has been immeasurable. Thanks go to the CDF group at Liverpool, to Tara Shears for helping with everything photon related, to Martin Griffiths for help with the CES and CPR plots, and to everyone for their input. Thanks also to the Photon and EWK groups at CDF for being very patient when listening to the very first talks I gave and providing an invaluable source of information. Many thanks go to Heather Gerberich, Ray Culbertson, Tara Shears for help with the fake rate measurement, I thought I would never get it! Thanks go to Robert Blair, David Dagenhart, Cigdem Issever and Ray again, for being an excellent godparent committee. Thanks Mike Houlden, Barry King and to everyone else who volunteered (or was volunteered!) to proof read this thesis at various stages. Thanks to PPARC for funding me for three years, and giving me the opportunity to work in Chicago for 18 months.

A final thank you to my friends and family for putting up with my stress! Thanks to my brothers, Debbie, Rish, Becky, Josie and Sallie to listening to my moans, and to all the fermi-posse, for making life out in Chicago so fantastic! Special thanks go to Aidan, Dustin, Tamsin, Emily, Ben, Anant, Sinéad, D0Si and Waschke. 


\section{Declaration}

No portion of the author's work described in this thesis has been submitted in support of an application for another degree or qualification in this, or any other, institute of learning. 


\section{Contents}

$\begin{array}{lll}1 & \text { Introduction } & 18\end{array}$

2 Theory 21

$2.1 Z^{0} \gamma$ production in the Standard Model and Beyond . . . . . . 21

2.1.1 Review of Standard Model . . . . . . . . . . . . . . . 22

2.1.2 Electroweak Unification . . . . . . . . . . . . . . . 24

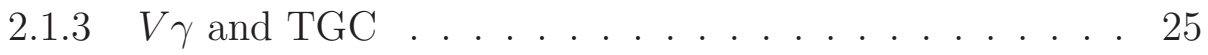

2.1.4 $W^{ \pm} \gamma$ and $Z^{0} \gamma$ production . . . . . . . . . . 26

2.2 Monte Carlo (MC) Simulation . . . . . . . . . . . . . . . . . . 31

2.2.1 Leading-Order Matrix Element Generators . . . . . . . 31

2.2 .2 k-factor . . . . . . . . . . . . . . . . . . 33

2.2.3 Uncertainty of SM Expectation . . . . . . . . . . 37

2.2.4 Cross-section prediction . . . . . . . . . . . . . 39

2.2.5 $Z^{0} \rightarrow l^{+} l^{-}$MC samples . . . . . . . . . . . . 39

3 The CDF Experiment at the Tevatron Collider 40

3.1 A Little History . . . . . . . . . . . . . . . . . . . . . . . 40

3.2 The Fermilab Accelerator Complex . . . . . . . . . . . . . . . 41

3.2.1 Proton Acceleration . . . . . . . . . . . . . . . . 41

3.2.2 Anti-proton Production . . . . . . . . . . . 44

3.3 The CDF Detector . . . . . . . . . . . . . . . . . 45

3.3.1 CDF Co-ordinate System and Related

Nomenclature . . . . . . . . . . . . . . . 45

3.3.2 Tracking Systems . . . . . . . . . . . . . . . . . . . 47

3.3.3 Time of Flight System . . . . . . . . . . . . . . . . 54

3.3.4 Calorimeter Systems . . . . . . . . . . . 55

3.3 .5 Muon Systems . . . . . . . . . . . . . . . 63

3.3.6 Cherenkov Luminosity Counters . . . . . . . . . . . 67

3.4 Trigger and Data Acquisition . . . . . . . . . . 70 
$4 Z^{0}$ boson Selection $\quad 71$

4.1 Event Vertex . . . . . . . . . . . . . . . . . . . 71

$4.2 \quad Z^{0} \rightarrow e^{+} e^{-}$Selection . . . . . . . . . . . . . . . 72

4.2.1 Electron Trigger Sample . . . . . . . . . . . . . 72

4.2.2 Good run list . . . . . . . . . . . . . . . . . 73

4.2 .3 Electron Identification Variables . . . . . . . . . . . 74

4.2.4 N-1 Plots . . . . . . . . . . . . . . . . . . . . 80

4.2.5 Electron Identification Efficiencies . . . . . . . . . . 84

4.2.6 $\mathbf{Z}^{\mathbf{0}} \rightarrow \mathbf{e}^{+} \mathbf{e}^{-}$Selection Efficiencies . . . . . . . . 84

$4.2 .7 Z^{0}$ selection . . . . . . . . . . . . . . 86

$4.3 Z^{0} \rightarrow \mu^{+} \mu^{-}$Selection . . . . . . . . . . . . . . . . . . . 88

4.3.1 Muon Trigger Samples . . . . . . . . . . . . . 88

4.3 .2 Good run list . . . . . . . . . . . . . . . . . . 89

4.3.3 Muon Identification Variables . . . . . . . . . . . . 90

$4.3 .4 \quad \mathrm{~N}-1$ plots . . . . . . . . . . . . . . . . . . . . . 94

4.3.5 Muon Efficiency . . . . . . . . . . . . . . . . . . 99

4.3.6 $\mathbf{Z}_{\text {CMUP }}^{0}$ and $\mathbf{Z}_{\mathbf{C M X}}^{\mathbf{n}}$ Selection Efficiencies . . . . . . . 100

4.3.7 $Z^{0}$ selection . . . . . . . . . . . . . . 103

5 Inclusive $\mathrm{Z}^{0}$ cross-section calculation $\quad 104$

5.1 Calibrations . . . . . . . . . . . . . . . . . . 104

5.2 Inclusive Acceptance and $\mathbf{Z}^{\mathbf{0}}$ Identification Efficiencies . . . . 105

5.3 Background Processes . . . . . . . . . . . . . . . . . . 109

5.3.1 Cosmic Rejection . . . . . . . . . . . . . . . . . 109

5.3.2 $Z^{0}$ Fake Background . . . . . . . . . . . . . . 109

5.4 Inclusive $Z^{0}$ and Cross-Sections . . . . . . . . . . . 111

6 Photon Identification $\quad 115$

6.1 Photon Selection . . . . . . . . . . . . . . . 115

6.1.1 Photon Identification Variables . . . . . . . . 116

$6.1 .2 \mathrm{~N}-1$ plots . . . . . . . . . . . . . . . . . . . . . . . 118

6.2 Photon Identification and

Reconstruction Efficiencies . . . . . . . . . . . . . . 118

6.2.1 Efficiency of Isolation variables . . . . . . . . . . . 122

6.2.2 Efficiency of Shower Shape Identification

Criteria . . . . . . . . . . . . . 125

6.2.3 Rate of Conversions . . . . . . . . . . . . . . . 126

6.2 .4 Efficiency Summary . . . . . . . . . . . . . . 126

$6.3 Z^{0} \gamma$ Acceptance and Identification Efficiencies . . . . . . . . . 127 
7 Estimation of Background Contributions $\quad 129$

7.1 Fake Rate Definition . . . . . . . . . . . . . . . . . . . . 130

7.2 Data Sample . . . . . . . . . . . . . . . . . . . . . . . 132

7.3 The Fake Probability . . . . . . . . . . . . . . . . . . 133

7.4 Contamination of the sample with True Photons . . . . . . . . 134

7.4.1 CES method . . . . . . . . . . . 136

7.4 .2 Iso vs. $\chi^{2}: \ldots \ldots \ldots \ldots . \ldots \ldots$

7.4.3 CPR method . . . . . . . . . . . . . . 141

7.4.4 Combining the three $F_{Q C D}$ Methods . . . . . . . . . . 143

7.5 The True Fake Probability . . . . . . . . . . . . . . . . . . . . 145

7.6 Fragmentation . . . . . . . . . . . . . . . 146

7.6.1 Application of Fake Rate . . . . . . . . . . . 147

7.7 Systematic Studies . . . . . . . . . . . . . . . . 147

7.7.1 $F_{\text {jet }}$ : See section $7.4 .4 \ldots \ldots . \ldots . \ldots 149$

7.7.2 Comparison of 2 nd to 345 th jet . . . . . . . . . . . 149

7.7.3 EM based fake rate . . . . . . . . . . . . 153

7.7.4 Effect of Varying Fragmentation and Smear . . . . . . 154

7.7.5 Effect of different Quark/Gluon mixtures . . . . . . . . 157

7.7.6 Conclusion of Systematic Studies . . . . . . . . . . . 161

7.8 Application of Method: Cross-Check . . . . . . . . . . . . . . 163

7.9 Predicted $Z^{0}+$ jet Background . . . . . . . . . . . . 165

7.10 Other Background Contributions . . . . . . . . . . . 165

8 Systematic Errors 170

9 Results $\quad 176$

9.1 Kinematic Distributions . . . . . . . . . . . . . . . 178

$9.2 Z^{0} \gamma$ Cross Sections . . . . . . . . . . . . . . . . . 178

9.3 Comparison to $W^{ \pm} \gamma \ldots \ldots \ldots \ldots$

$\begin{array}{ll}10 \text { Summary and Outlook } & 187\end{array}$

$\begin{array}{ll}\text { A Appendix } & 188\end{array}$

A.1 Lepton identification efficiencies . . . . . . . . . . . . . 188

A.2 Additional Fake Rate Studies . . . . . . . . . . . . . . . . . . 190

A.2.1 Isolation v. $\chi^{2}$ independence . . . . . . . . . . 190

A.2.2 Boundary choice for CES method . . . . . . . . . . 190

A.3 Other Studies . . . . . . . . . . . . . . . . . . . . . 190

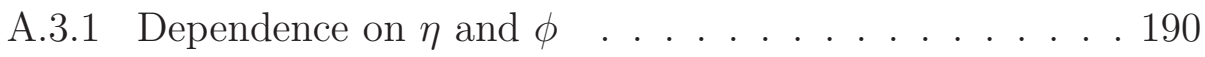




\section{List of Tables}

2.1 Lepton masses, charge and spin [2]. . . . . . . . . . . . . . 22

2.2 Approximate quark masses, charge and spin. . . . . . . . . . 23

2.3 A summary table of the fundamental forces of nature and the properties of their mediating gauge bosons. (*postulated) . . . 23

2.4 The $95 \%$ C.L. intervals obtained combining the results from ALEPH, DELPHI and L3. In each case the two parameters listed are varied while the remaining ones are fixed to the SM values. Both the statistical and systematic uncertainties are included. [6] . . . . . . . . . . . . . . . 30

2.5 The $95 \%$ C.L. intervals obtained combining the results from D0, for a form factor scale $\Lambda=750 \mathrm{GeV}$. In each case, only one coupling is allowed to be non-zero. Both the statistical and systematic uncertainties are included.[16] . . . . . . . 30

2.6 Parameters used in ZGAMMA and WGAMMA . . . . . . . . . 34

2.7 Parton level cuts used for final $Z^{0} \gamma$ samples . . . . . . . . . . 34

2.8 Comparison between ZGAMMA and MADGRAPH. A flat " $k$ "-factor of 1.365 has been applied to all samples (see section 2.2.2). . . 36

2.9 Systematic errors on the $Z^{0} \gamma \mathrm{MC}$ generation . . . . . . . . . . 38

3.1 Luminosities obtained for different data collecting runs at the Tevatron. . . . . . . . . . . . . . 41

3.2 Design parameters of the tracking systems [30] . . . . . . . 52

3.3 Description of the Central Preradiator Chambers [45] . . . . . 59

3.4 The transverse tower segmentation of the upgraded plug calorimeter. [30]. . . . . . . . . . . . . . . . . . . . 62

3.5 Design parameters of the CDF muon detectors. Pion interaction lengths and multiple scattering are computed at a reference angle of $\theta=90^{\circ}$ in $\mathrm{CMU}$ and CMP, and at an angle of $\theta=55^{\circ}$ in the CMX [30]. . . . . . . . . . . . . 65

4.1 ELECTRON_CENTRAL_18 Trigger requirements. . . . . . . . . . 73 
4.2 L3 Trigger Path, Dataset name and integrated luminosity $(\mathcal{L})$ for the electron channels. There is a $6 \%$ systematic error quoted on the luminosity, see section 3.3.6. . . . . . . . . 74

4.3 Table of cuts to select tight and loose central electrons. . . . 79

4.4 Criteria used in the selection of the plug electron. . . . . . . . 79

4.5 A summary of the efficiencies and correction factors used for the $Z_{C C}^{0}$ cross-section calculation. Details about $\epsilon_{\text {tight }}$ and $\epsilon_{\text {loose }}$ are found in [61]. The total correction factor is the product of the terms in the right column. . . . . . . . . . 87

4.6 A summary of the efficiencies and correction factors used for the $Z_{C P}^{0}$ cross-section calculation. Details about $\epsilon_{p l u g}$ are found in [64]. The total correction factor is the product of the terms in the right column. . . . . . . . . . . . . 87

4.7 CMUP_18 Trigger requirements. . . . . . . . . . . . . . . . . 89

4.8 CMX_18 Trigger requirements. . . . . . . . . . . . . . . . . . . 90

4.9 L3 Trigger Path, Dataset name and integrated luminosity $(\mathcal{L})$ for the muon analysis channels. There is a $6 \%$ systematic error quoted on the luminosity, see section 3.3.6. . . . . . . . . 90

4.10 Table of cuts to select tight and loose muons. No explicit cut on the tight muon $\eta$ is made but due to the requirement of a CMU or CMX stub, this corresponds to cut on detector $|\eta|<1.0 . \ldots \ldots \ldots \ldots$

4.11 A summary of the efficiencies, in data and $\mathrm{MC}$, and correction factors, $\left(\epsilon_{\text {Data }} / \epsilon_{M C}\right)^{l}$, used for the $Z_{C M U P}^{0}$ cross-section calculation. . . . . . . . . . . . . . . . 100

4.12 A summary of the efficiencies, in data and MC, and correction factors, $\left(\epsilon_{\text {Data }} / \epsilon_{M C}\right)^{l}$, used for the $Z_{C M X}^{0}$ cross-section calculation. . . . . . . . . . . . . . . . . 101

4.13 A summary of the efficiency correction used for the $Z_{C M U P / X}^{0}$ cross-section calculation. . . . . . . . . . . . 102

5.1 Run dependent energy calibrations for the east and west plugs. There is a $0.3 \%$ error on the calibrations used, determined from the calibration of the data to the PDG value of the $Z^{0}$ boson mass. . . . . . . . . . . . . . . . . . . 105

5.2 Acceptance $\times$ efficiency for the $Z^{0} \rightarrow e^{+} e^{-}$analyses. . . . . 108

5.3 Acceptance $\times$ efficiency for the $Z^{0} \rightarrow \mu^{+} \mu^{-}$analyses. . . . . 108

5.4 Summary table of values used for inclusive cross-section measurement. The errors on the various quantities are given as a percentage in brackets . . . . . . . . . . . . . 113

5.5 The measured $\sigma\left(Z^{0}\right) \times \mathrm{BR}\left(\mathrm{l}^{+} \mathrm{l}^{-}\right) \ldots \ldots \ldots . . \ldots . \ldots 114$ 
6.1 The list of photon selection cuts, with a brief note to categorise the cut to whether it is a geometry, isolation or shower shape requirement. . . . . . . . . . . . . . . . . . 119

6.2 $W^{ \pm}$selection cuts for random cone studies. $M_{T}(e, \nu)$ is the transverse mass calculated from the vectors associated with the electron and missing transverse energy, $\mathbb{E}_{T}: M_{T}\left(e, \mathbb{E}_{T}\right)=$ $\sqrt{2 p_{T}^{e} \mathbb{E}_{T}\left(1-\cos \phi_{e} \Phi_{T}\right)} \ldots \ldots \ldots \ldots$

6.3 Correction factors used to scale the MC in data [74]. The calorimeter isolation depends on $E_{T}^{\gamma} \cdot f\left(E_{T}\right)=0.89+0.0175 \times$ $E_{T}-0.00104 \times E_{T}^{2}+2 \times 10^{-5} \times E_{T}^{3}$ for $E_{T}<20 \mathrm{GeV}$, and $f\left(E_{T}\right)=0.98$ for $E_{T}>20 \mathrm{GeV} \ldots \ldots \ldots \ldots . \ldots . \ldots 124$

6.4 The summary of the correction factor to scale the $Z \gamma$ MC. $f\left(E_{T}\right)=0.89+0.0175 \times E_{T}-0.00104 \times E_{T}^{2}+2 \times 10^{-5} \times E_{T}^{3}$ for $E_{T}<20 \mathrm{GeV}$, and $f\left(E_{T}\right)=0.98$ for $E_{T}>20 \mathrm{GeV}$. . . . 125

6.5 Acceptance $\times$ Efficiency for $Z^{0} \gamma \rightarrow e^{+} e^{-} \gamma$ and $Z^{0} \gamma \rightarrow \mu^{+} \mu^{-} \gamma 128$

7.1 Cut values to define the regions used for the Iso vs. $\chi^{2}$ method

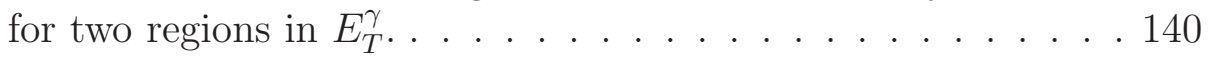

7.2 The number of $Z^{0}+$ jet background events in the different $Z^{0} \gamma$ channels. . . . . . . . . . . . . . . . 165

7.3 The number of same sign events found for the background calculation of equation 7.25. The errors shown are statistical errors only in the MC prediction. . . . . . . . . . . . . 168

7.4 The loose $Z^{0} \rightarrow e^{+} e^{-}$selection used for estimating the fake $Z_{C C}^{0}$ plus $\gamma$ contribution. . . . . . . . . . . . . . 168

$8.1 Z^{0} \gamma$ selection for acceptance systematic . . . . . . . . . . . . 173

8.2 A summary of all systematic errors considered for the $Z^{0} \gamma$ cross-section and their correlations between the $Z_{C C}^{0}, Z_{C P}^{0}$, $Z_{C M U P}^{0}$ and $Z_{C M X}^{0}$ channels. An "x" indicates the uncertainty is applied to that channel and thus correlated to all the other channels with the same systematic. If a different value is applied to the $Z_{C P}^{0} \gamma$, due to only one lepton in the central region of the detector, its value is indicated. . . . . . . . . . . . 174

9.1 Numbers of events in data and those expected for $Z^{0} \gamma \rightarrow$ $e^{+} e^{-} \gamma$ and $Z^{0} \gamma \rightarrow \mu^{+} \mu^{-} \gamma \ldots \ldots \ldots \ldots$. . . . . . . . . 177

9.2 Numbers of events in data and those expected for $Z^{0} \gamma \rightarrow$ $l^{+} l^{-} \gamma$, after combining the separate channels. . . . . . . . 177

9.3 Lepton $E_{T}$ and $\mathbb{E}_{T}$ cuts used for the different $W^{ \pm} \gamma$ channels. . 186 
A.1 N-1 efficiencies for the central electron identification variables. [61] . . . . . . . . . . . . . . . . . . . . 188

A.2 N-1 efficiencies for the plug electron identification variables. (excluding PHOENIX track requirement [64] . . . . . . . . . 189

A.3 N-1 efficiencies for CMUP muon identification variables[58] . . 189

A.4 N-1 efficiencies for CMX muon identification variables[58]. The CMX stub efficiency assumes the $\rho$ cut has already been made. 189

A.5 The variance of $F_{Q C D}$ on the choice of boundaries for method 7.4.1: $\mathrm{IsoC}=($ Iso4-2) $/(20-\mathrm{Et}) \ldots \ldots . \ldots . . \ldots 191$ 


\section{List of Figures}

2.1 Tree-level diagrams for $Z^{0} \gamma$ and $W^{ \pm} \gamma$ production . . . . . . . 27

2.2 The generated invariant mass distributions for the leading order $Z^{0} \gamma$ processes produced from MC simulation. . . . . . . . 27

2.3 Trilinear $Z V \gamma$ vertex in $q \bar{q} \ldots \ldots . \ldots . \ldots . . \ldots 28$

2.4 Virtual and soft gluon radiation, QCD corrections to the initial state radiation $Z^{0} \gamma$ processes. Not shown are the diagrams obtained by interchanging the $Z^{0}$ and the $\gamma \cdot[11] \ldots 35$

2.5 The k-factor correction for $Z^{0} \gamma$. This function is applied to events with $m(l, l)>86 \mathrm{GeV} / c^{2}$. The inclusive $Z^{0} \mathrm{NLO}$ correction of 1.36 (shown as straight line), is applied to the inner bremstrahlung events. . . . . . . . . . . . 37

3.1 The Fermilab accelerator complex. . . . . . . . . . . . . . 42

3.2 Cross-section view of CDF in the r-z view (see section 3.3.1). . 46

3.3 The CDF coordinate system. . . . . . . . . . . . 47

3.4 The cross-section of SVXII and ISL tracking systems. . . . . . 49

3.5 Schematic diagram of the arrangement of the 8 axial and stereo superlayers comprising the Central Outer Tracker. . . . . . . . 50

3.6 An example of a COT drift cell in Superlayer 2 . . . . . . . 51

3.7 Tracking efficiencies of the different tracking algorithms. . . . 55

3.8 Schematic of a typical central electromagnetic calorimeter wedge. 58

3.9 Schematic, head on view, of a CPR cell. Shown are the ground (g) and signal (s) wires. . . . . . . . . . . . . 60

3.10 Cross-section view of top half of plug calorimeter . . . . . . 61

3.11 Schematic diagram of one of the eight PES sectors showing the direction of the strips in the $U$ and $V$ layers. . . . . . . . 63

3.12 Location of the central muon detectors in azimuth $\phi$ and pseudorapidity $\eta$. On the east side, there is a gap in coverage in the CMX of $30^{\circ}$ in azimuth, due to the location of the cryogenic utilities servicing the solenoid. 
3.13 A CMU module in the $r-\phi$ plane with 4 layers of drift chambers. The drift times $t_{1}$ and $t_{2}$ are used to calculate muon momentum for triggering. Shown as a black circles, are the sense wires connected to the readout. . . . . . . . . 66

3.14 Side view of the CDF detector showing the placement of the CMX detectors. . . . . . . . . . . . . . . . . . . 68

3.15 The relative production cross-sections for different processes at CDF. . . . . . . . . . . . . . . .

4.1 N-1 distributions for central electron variables used for the selection of the events, plotted for data (dots) and MC (solid histogram). The arrow shows graphically the selection cut. The MC simulation is normalised to the luminosity of the data. 82

$4.2 \mathrm{~N}-1$ distributions for central electron variables used for the selection of the events, plotted for data (dots) and MC (solid histogram). The arrow shows graphically the selection cut. The MC simulation is normalised to the luminosity of the data. 83

$4.3 \mathrm{~N}-1$ distributions for plug electron variables used for the selection of the events, plotted for data (dots) and MC (solid histogram). The arrow shows graphically the selection cut. The MC simulation is normalised to the luminosity of the data. 85

4.4 The exit radius $(\rho)$ of particles exiting the COT can be calculated from the $\theta$ and $z_{0}$ of the track. . . . . . . . 93

4.5 Illustration of the muon $z$ fiducial distance. The $z(x)$-fiducial distance is defined to be the distance between the extrapolated COT track and the edge of the muon chamber, in the direction parallel (perpendicular) to the drift wire. The convention is used whereby tracks which extrapolate to the outside (inside) of the chamber have fiducial distances greater (less) than 0. . . 93

4.6 N-1 distributions for muon identification variables used for the selection of the events, plotted for data (dots) and MC (solid histogram). The arrow shows graphically the selection cut. The MC simulation is normalised to the luminosity of the data. 97

4.7 N-1 distributions for muon identification variables used for the selection of the events, plotted for data (dots) and MC (solid histogram). The arrow shows graphically the selection cut. The MC simulation is normalised to the luminosity of the data. 98

5.1 Comparison of the invariant mass of $Z_{C C}^{0}$ candidate events in the data to $Z^{0} \rightarrow e^{+} e^{-}$MC . . . . . . . . . . . . . . . . 106 
5.2 Comparison of the invariant mass of $Z_{C P}^{0}$ candidate events in the data to $Z^{0} \rightarrow e^{+} e^{-}$MC . . . . . . . . . . . . . . . 106

5.3 Comparison of the invariant mass of $Z_{C M U P}^{0}$ candidate events in the data to $Z^{0} \rightarrow \mu^{+} \mu^{-}$MC . . . . . . . . . . . . 106

5.4 Comparison of the invariant mass of $Z_{C M X}^{0}$ candidate events in the data to $Z^{0} \rightarrow \mu^{+} \mu^{-}$MC . . . . . . . . . . . . . 106

5.5 The invariant mass of $Z_{C C}^{0}$ candidate events as a function of the integrated luminosity. . . . . . . . . . . . . 107

5.6 The invariant mass of $Z_{C P}^{0}$ candidate events as a function of the integrated luminosity of the $Z_{C C}^{0}$ sample. . . . . . . . 107

5.7 The invariant mass of $Z_{C M U P}^{0}$ candidate events as a function of the integrated luminosity of the $Z_{C C}^{0}$ sample. . . . . . . 107

5.8 The invariant mass of $Z_{C M X}^{0}$ candidate events as a function of the integrated luminosity of the $Z_{C C}^{0}$ sample. . . . . . . 107

5.9 Fake Rate for a jet to fake a central tight electron. Shown is a fit to the data. . . . . . . . . . . . . . . . 111

5.10 Fake Rate for a jet to fake a plug electron for the east (top plot) and west plugs (bottom plot). Shown are the fits to the data. . . . . . . . . . . . . . . . . 112

6.1 N-1 distributions for photon identification variables used for the selection of the events, plotted for data (dots) and MC (solid histogram). The arrow shows graphically the selection cut, where appropriate. The MC simulation is scaled to the luminosity of the data. . . . . . . . . . . . . . . . 120

6.2 N-1 distributions for photon identification variables used for the selection of the events, plotted for $e^{+} e^{-} \gamma$ and $\mu^{+} \mu^{-} \gamma$ data (dots) and MC (solid histogram). The arrow shows graphically the selection cut, where appropriate. The MC simulation is scaled to the luminosity of the data. . . . . . . . . . . . . . 121

6.3 The isolation efficiency is plotted versus the assumed photon energy in $W^{ \pm} \rightarrow e^{ \pm} \nu$ data and Monte Carlo events. The data is for any number of vertices, while the Monte Carlo has only one vertex.

7.1 The $E_{T}^{\text {jet }}$ distribution $\mathrm{d} N / \mathrm{d} E_{T}^{\text {jet }}$ for jets in the $Z^{0}$ sample (black points), the 2 nd jet in the jet samples (blue squares) and the 345th jet in the jet samples (red open triangles). . . . . . . . 131

7.2 The $\Delta R$ distribution between the prompt $\gamma$ candidate and any "jet' in ZGAMMA MC. . . . . . . . . . . . . . . . . . . 133 
$7.3 P_{\text {raw }}$ versus jet $E_{T}$ comparing $J E T \_20$ (open points) and JET_50, JET_70 and JET_100 data (full points). . . . . . . . . . 135

7.4 The signal $\left(\epsilon_{\gamma}\right)$ and background $\left(\epsilon_{b}\right)$ templates for the CES method . . . . . . . . . . . . . . . 139

7.5 I so4 $/ E_{T}$ (left) and (Iso4 -2$) /\left(20-E_{T}^{\gamma}\right)$ versus $\chi^{2}$. Illustrated are the four regions $\mathrm{A}, \mathrm{B}, \mathrm{C}$ and $\mathrm{D}$ used for determining the QCD background fraction $F_{\text {jet }}($ see table 7.1$) \ldots . . . . .140$

7.6 The conversion probabilities for signal prompt photons $\left(P_{c o n v}^{\gamma}\right)$, and background mesons $\left(P_{c o n v}^{Q C D}\right)$ as a function of $E_{T}$. . . . . 143

7.7 $F_{j e t}$ as a function of $E_{T}^{\text {jet }}$, as measured using the three independent methods for the 345th jet sample. "Comb" denotes the combination of the three methods as discussed in the text. The data are compared to the final fit (solid line) of the average of the "Iso-CES" and "CES" for $E_{T}^{\gamma}<40 \mathrm{GeV}$ and the $\mathrm{CPR}$ for $E_{T}^{\gamma}>40 \mathrm{GeV}$. The upper and lower errors on the fit are also shown (dashed lines). . . . . . . . . . . . . . . 144

7.8 Shown is the ratio of the $F_{\text {jet }}$ determinations (closed point: Iso vs CES, open points: CES, triangles: CPR) to the final value used and the systematic error (shaded ares) and the statistical errors of the individual methods. . . . . . . . . . . . . . . 145

7.9 The corrected "true" fake rate $P_{\text {jet } \rightarrow \gamma}\left(E_{T}^{\text {jet }}\right)$ for the 345 th jet sample. The result is shown on a linear (left) and logarithmic scale (right). . . . . . . . . . . . . . . . . 146

7.10 The distribution of $Z=E_{T}^{\gamma} / E_{T}^{j e t}$ in the jet triggered data samples. Shown is a Gaussian fit to the distribution. . . . . . 147

7.11 Dependence of a) the mean value and b) the resolution of $Z^{0}$ on $E_{T}^{j e t}$. The dashed lines indicates the average values of the full sample. . . . . . . . . . . . . . . . . . . . 148

7.12 Feynman diagrams for photon production. a) and b) show the prompt photon production diagrams and c) a final state radiation example diagram. . . . . . . . . . . . . . 150

7.13 Fractional contribution of quark and gluon jets for the 2 nd jet in the PYTHIA jet MC. . . . . . . . . . . . . . . . . . 151

$7.14 P_{\text {raw }}\left(E_{T}^{\text {jet }}\right)$ for 2 nd jet sample versus $E_{T}^{\text {jet }} \ldots \ldots \ldots \ldots 1$

$7.15 F_{\text {jet }}$ for 2 nd jet sample versus $E_{T}^{\text {jet }} \ldots \ldots \ldots \ldots$. . . . . . 152

$7.16 P_{\text {jet } \rightarrow \gamma}\left(E_{T}^{\text {jet }}\right)$ for 2 nd jet sample versus $E_{T}^{\text {jet }}$. Shown for comparison is the function for $P_{\text {jet } \rightarrow \gamma}\left(E_{T}^{\text {jet }}\right)$ calculated in the 345 th jet sample. . . . . . . . . . . . . . . . . 152

$7.17 P_{\text {raw }}\left(E_{T}^{\text {jet }}\right)$ for EM objects versus $E_{T}^{E M} \ldots \ldots \ldots \ldots \ldots$

$7.18 F_{E M}$ for EM objects versus $E_{T}^{E M} \ldots \ldots \ldots \ldots \ldots$

$7.19 P_{\text {jet } \rightarrow \gamma}\left(E_{T}^{\text {jet }}\right)$ for EM objects versus $E_{T}^{E M} \ldots \ldots \ldots \ldots$ 
7.20 The QCD predicted background for the $W^{ \pm} \gamma$ (left) and $Z^{0} \gamma$ (right) samples using the jet based fake rate method (closed points) and the EM fake rate (open points). Also shown is the EM fake rate prediction after subtracting the expected contaminations from $W \gamma$ and $Z^{0} \gamma$ signal (histogram). . . . 156

$7.21 P_{T}$ distribution of highest $P_{T} \pi^{0}$ in gluon and quark jets in PYTHIA jet MC. Although there are more gluon jets present, their $P_{T}$ spectrum is much softer. . . . . . . . . . . . . 158

7.22 Fake rate for quark and gluon jets for jets containing a hard $\pi^{0}$ or $\eta$ in HERWIG jet MC. . . . . . . . . . . . . . . . . . . . 159

7.23 Fractional contribution of quark and gluon jets in the PYTHIA dijet MC (left) and the ALPGEN $Z+1$ jet MC. . . . . . . . . . 159

$7.24 P_{\text {jet } \rightarrow \gamma}^{j}\left(E_{T}^{\text {jet }}\right)$ as measured in PYTHIA and HERWIG MC samples. For Comparison purposes, $P_{\text {jet } \rightarrow \gamma}^{j}\left(E_{T}^{\text {jet }}\right)$ measured from the 345th data sample is also shown. The MC samples have approximately a 40\% statistical error. . . . . . . . . . . 161

7.25 Fractional systematic error on the fake rate due to: a) the method for statistical precision on $F_{j e t}$, b) the fake rate determined from 2 nd jet sample, c) the fake rate determined from using EMobjects as the denominator, d) the fake rate determined from varying the fragmentation. . . . . . . . . . 162

7.26 Figure a shows the fractional systematic error on the fake rate due to the quark/gluon content of jet sample. In Figure b, the quadratic sum of the five components shown in figures 7.25 and 7.26a is shown. . . . . . . . . . . . . 163

7.27 The observed fake photon spectrum compared to the fake rate prediction for the JET_20 (a), JET_50 (b), JET_70 (c) and JET_100 (d) datasets. . . . . . . . . . . . . . . . . 164

7.28 The observed fake photon spectrum compared to the fake rate prediction for the JET_20 (a), JET_50 (b), JET_70 (c) and JET_100 (d) datasets, on a log scale. . . . . . . . . . 166

8.1 The two $E / P$ distributions of electrons from the decay of a $J / \Psi$, in data and $\mathrm{MC}$, agree to within $1 \%$. . . . . . . 172

9.1 Photon $E_{T}$ spectrum for CC(top-left), CP(top right), $\mu \mu$ (bottom left) and combined $Z^{0} \gamma$ (bottom right) channels . . . . . . . . 179

9.2 Photon $E_{T}$ spectrum, on a log scale, for CC(top-left), CP(top right), $\mu \mu$ (bottom left) and combined $Z^{0} \gamma$ (bottom right) channels . . . . . . . . . . . . . . . . . . . 180 
9.3 $\Delta R_{l \gamma}$ distribution between the photon and closest lepton for $\mathrm{CC}$ (top-left), $\mathrm{CP}$ (top right), $\mu \mu$ (bottom left) and combined $Z^{0} \gamma($ bottom right) channels . . . . . . . . . . . . . . 181

9.4 The dilepton invariant mass distribution for CC(top-left), $\mathrm{CP}$ (top right), $\mu \mu$ (bottom left) and combined $Z^{0} \gamma$ (bottom right) channels . . . . . . . . . . . . . . . . . . . . . . 182

9.5 The three-body invariant mass distribution for CC(top-left), $\mathrm{CP}$ (top right), $\mu \mu$ (bottom left) and combined $Z^{0} \gamma$ (bottom right) channels . . . . . . . . . . . . . . . . . 183

9.6 Invariant mass of the two leptons and the photon versus the di-lepton invariant mass for $Z^{0} \gamma$ candidates in the electron (circle) and muon (triangle) channels. The black dots are the expectations from the $Z^{0} \gamma$ MC . . . . . . . . . . . . 184

A.1 CES $\chi^{2}$ distribution for fake photon candidates in HERWIG dijet MC simulation. Candidates which pass and fail the Iso4 cut are plotted separately. . . . . . . . . . . . . 190

A.2 The uncorrected fake rate as a function of $\eta$ and $\phi \ldots . . . .191$ 


\section{Chapter 1}

\section{Introduction}

In order to further our understanding of the fundamental constituents and forces of nature, it sometimes seems ironic that we need some of the largest experimental apparatus in the world. Studying the output of highly energetic collisions is one of our most useful tools to understand not only how the universe works today, but gives insight into the universe in its early life, just after the big bang.

One of the great advances in particle physics in the last 40 years is our understanding of the Weak interaction. In the period 1961-67, the ElectroWeak (EWK) theory[3, 4, 5] was developed, by Sheldon Glashow, Steven Weinberg and Abdus Salam. This theory unifies the electromagnetic and weak forces, into two aspects of the same interaction. It predicted the existence of four particles: two charged $\left(W^{ \pm}\right)$, one neutral $\left(Z^{0}\right)$ of large mass and one neutral of 0 mass $(\gamma)$. The great experimental advances followed soon after. In the late 1960's a bubble chamber, known as Gargamelle, was built at the Saclay Laboratory in France. In early 1970's neutral current (i.e. $Z^{0}$ exchange) neutrino scattering was observed, but it took another 10 years before the $W^{ \pm}$ 
and $Z^{0}$ bosons were discovered at the UA1 experiment. The LEP experiment at CERN carried out precision measurements on both the mass and width of the $Z^{0}$ boson, making it one of the most accurately measured and well understood particles of the Standard Model (SM).

The EWK theory gives us a prediction of how the $W^{ \pm}, Z^{0}$ and $\gamma$ bosons interact with each other. The first evidence of self-coupling was observed at the LEP experiment for the $W W \gamma$ vertex[6]. Careful examination of the self-couplings of these bosons is a good test of the EWK theory and any deviations of these couplings from those predicted would provide indications of new physics.

The CDF experiment at Fermilab gives an opportunity to explore interactions at higher centre-of-mass energies in proton-antiproton collisions. CDF and its sister experiment, D0, have already placed world leading measurements on many aspects of the SM. For example, the discovery of the top quark and precision measurements of its mass and the mass of the $W^{ \pm}$.

In this thesis a measurement of the $Z^{0} \gamma$ cross-section is made for the kinematic range $\Delta R_{l \gamma}>0.7, E_{T}^{\gamma}>7 \mathrm{GeV}$ and $\mathrm{m}(\mathrm{l}, \mathrm{l})>40 \mathrm{GeV} / \mathrm{c}^{2}$. using 202 $\mathrm{pb}^{-1}$ of data collected from $p \bar{p}$ collisions at the Collider Detector at Fermilab (CDF). The results obtained in this thesis have been submitted to Physics Review Letters [1].

I have developed a jet-photon fake rate determination method, which is available for and has been used by other analyses at CDF. I worked on implementing the calorimeter seeded tracking algorithm into the standard CDF software.

A theoretical overview of EWK physics is given in chapter 2. A descrip- 
tion of the $Z^{0} \gamma$ process and triple gauge boson coupling is also given. The influence of possible contributions from beyond the Standard Model is briefly described. In chapter 3, the Tevatron and the CDF detector, the experimental apparatus used for this measurement are described. The selection of $Z^{0} \gamma$ events, is described in chapters 4 and 6 , with chapter 4 giving a description of the $Z^{0}$ selection. The additional requirement of the $\gamma$ is given in chapter 6. The background sources are discussed in chapter 7, with the major contribution estimated using a jet-photon fake-rate technique. The systematic errors for this analysis are discussed and summarised in chapter 8. The results are finally presented in chapter 9 , and the kinematic distributions and the cross-sections presented with the next-to-leading-order Standard Model prediction. 


\section{Chapter 2}

\section{Theory}

A brief review of the Standard Model and a description of the part played by $Z^{0} \gamma$ production and the triple gauge couplings, $Z Z \gamma$ and $Z \gamma \gamma$, is given in section 2.1. Section 2.2 discusses the Monte Carlo simulation used to provide next-to-leading order (NLO) prediction of $Z^{0} \gamma$ production for this analysis.

\section{1 $Z^{0} \gamma$ production in the Standard Model and Beyond}

The Standard Model (SM) makes precise predictions for the self couplings of gauge bosons. These self-interactions are described by the triple gauge boson couplings $W W V, Z \gamma V$ and $Z Z V\left(Z \equiv Z^{0}, W \equiv W^{ \pm}\right.$and $\left.V=\gamma, Z\right)$. The measurement of vector boson production such as $Z^{0} \gamma$ production, provides a sensitive direct test of the trilinear couplings, and any deviations from the $\mathrm{SM}$ values would be an indication of new physics. 


\begin{tabular}{|l|c|c|c|}
\hline lepton & mass $\left(\mathrm{MeV} / c^{2}\right)$ & charge & spin \\
\hline electron $\left(e^{-}\right)$ & 0.511 & -1 & $1 / 2$ \\
electron neutrino $\left(\nu_{e}\right)$ & $<3 \times 10^{-6}$ & 0 & $1 / 2$ \\
\hline muon $\left(\mu^{-}\right)$ & 105.66 & -1 & $1 / 2$ \\
muon neutrino $\left(\nu_{\mu}\right)$ & $<0.19$ & 0 & $1 / 2$ \\
\hline tau $\left(\tau^{-}\right)$ & 1777 & -1 & $1 / 2$ \\
tau neutrino $\left(\nu_{\tau}\right)$ & $<18.2 \times 10^{-6}$ & 0 & $1 / 2$ \\
\hline
\end{tabular}

Table 2.1: Lepton masses, charge and spin [2].

\subsubsection{Review of Standard Model}

In the SM, all matter in the universe is made up of point-like particles called quarks and leptons. Each particle has a partner "anti-particle" which has the same mass but opposite quantum numbers. The SM includes description of three out of the four fundamental forces of nature: electromagnetism, the strong and the weak force. The fourth, gravity is many orders of magnitude weaker than the others for the energies and distances available in the laboratory and can be safely ignored.

The fundamental particles of the SM can be placed into three families: leptons, quarks and gauge bosons. There are three families of leptons and three of quarks. Each lepton family consists of one charged particle $\left(l^{ \pm}\right)$and a neutral $\left(\nu_{l}\right)$ particle with negligable mass called a neutrino. The properties of the lepton families are sumarised in table 2.1.

The three families of quarks are summarised in table 2.2. In addition to electromagnetic charge, quarks also carry a colour charge, associated with the strong force. A proton contains three valence quarks and a sea of virtual 


\begin{tabular}{|l|c|c|c|c|}
\hline Quark (q) & Quantum number & Rest Mass $\left(\mathrm{GeV} / c^{2}\right)$ & charge & spin \\
\hline up (u) & - & 0 & $2 / 3$ & $1 / 2$ \\
down (d) & - & 0 & $-1 / 3$ & $1 / 2$ \\
\hline strange (s) & $\mathrm{S}=-1$ & 0.50 & $-1 / 3$ & $1 / 2$ \\
charm (c) & $\mathrm{C}=+1$ & 1.6 & $+2 / 3$ & $1 / 2$ \\
\hline bottom (b) & $\mathrm{B}=-1$ & 4.6 & $-1 / 3$ & $1 / 2$ \\
top (t) & $\mathrm{T}=+1$ & 178 & $+2 / 3$ & $1 / 2$ \\
\hline
\end{tabular}

Table 2.2: Approximate quark masses, charge and spin.

\begin{tabular}{|l|c|c|c|c|}
\hline Force & Gauge Boson & Rest Mass $\left(\mathrm{GeV} / c^{2}\right)$ & charge & spin \\
\hline Electromagnetism & photon $(\gamma)$ & 0 & 0 & 1 \\
Strong & gluon $(\mathrm{g})$ & 0 & 0 & 1 \\
Weak & $W^{ \pm}, Z^{0}$ & $80.4,91.0$ & $\pm 1,0$ & 1 \\
Gravity & graviton* & 0 & 0 & 2 \\
\hline
\end{tabular}

Table 2.3: A summary table of the fundamental forces of nature and the properties of their mediating gauge bosons. (*postulated)

$q \bar{q}$ pairs and gluons.

Quarks and leptons, like all charged particles, have a corresponding antiparticle with the same mass but opposite charge.

Each of the four fundamental forces of nature is mediated by a particle known as a "gauge boson". The electromagnetism force is mediated by the photon, the strong force by the gluon, the weak force by the $Z^{0}$ and $W^{ \pm}$ bosons and gravity by the postulated graviton. Some properties of the gauge bosons are summarised in table 2.3.

The last piece of the Standard Model, which is as of yet undiscovered, 
is the Higgs boson which is believed to be responsible for giving the other particles of the SM their mass through interactions with the Higgs. This particle is predicted to have spin 0 but its mass is only loosely constrained $[7,8,9]$.

\subsubsection{Electroweak Unification}

According to the theory of Glashow, Weinberg and Salam $[3,4,5]$ the electromagnetism and weak forces are two manifestations of a single electroweak force with a single coupling given by the electric charge e. They made the prediction that this symmetry would only be apparent at large momentum transfers $\left(Q^{2} \approx M_{W}^{2}\right)$. At lower energies the symmetry would be broken. Of the four mediating vector bosons involved, one (the photon) would be massless, and the others $\left(W^{ \pm}, Z^{0}\right)$ would be massive. One immediate result of this is that weak interactions are much weaker and shorter distance compared to the electromagnetic force.

In the electroweak theory, the four gauge fields are arranged in a triplet of 'weak isospin' $I$ and a singlet of 'weak hypercharge' Y. They are: $W_{\mu}^{i}$ $(i=1,2,3)$, belonging to the $I=1$ triplet of the group $S U(2)_{L}$, and $B_{\mu}$, an isoscalar $I=0$ belonging to the $U(1)_{Y}{ }^{1}$. group of weak hypercharge. The weak hypercharge is related to the electric charge $(\mathrm{Q})$ and the weak isospin by the Gell-Mann-Nishijima relation: $Q=I_{3}+Y / 2\left(I_{3}\right.$ is the 3 -projection of I).

The electroweak bosons are combinations of the $W_{\mu}^{i}$ and $B_{\mu}$ fields. The

\footnotetext{
${ }^{1}$ Where $L$ and $Y$ denotes that only left handed particles transform under the $S U(2)$ weak isospin symmetry group and both right and left handed particles transform under the $\mathrm{U}(1)$ weak hypercharge group respectively.
} 
$W_{\mu}^{ \pm}$charged particles are directly related to the $W_{\mu}^{1}$ and $W_{\mu}^{2}$ fields. The neutral particles $A_{\mu}$ (the photon) and $Z_{\mu}$ (the $Z^{0}$ ) are taken as combinations of $W_{\mu}^{3}$ and $B_{\mu}$ fields (where $\theta_{w}$ is the weak mixing angle of the $\mathrm{SM}$ ):

$$
\begin{aligned}
& Z_{\mu}=\cos \theta_{w} W_{\mu}^{3}-\sin \theta_{w} B_{\mu} \\
& A_{\mu}=\sin \theta_{w} W_{\mu}^{3}+\cos \theta_{w} B_{\mu}
\end{aligned}
$$

\subsection{3 $V \gamma$ and TGC}

The Lagrangian of the EWK model is the sum of four parts, the lagrangians for the gauge fields $\left(\mathcal{L}_{W}\right)$, the fermion fields $\left(\mathcal{L}_{f}\right)$, the scalar fields $\left(\mathcal{L}_{s}\right)$ and finally the lagrangian for the coupling between the fermions and the scalar fields $\left(\mathcal{L}_{f-s}\right)$ :

$$
\mathcal{L}=\mathcal{L}_{W}+\mathcal{L}_{f}+\mathcal{L}_{s}+\mathcal{L}_{f-s}
$$

The description of the trilinear and quadralinear gauge couplings of the gauge boson fields are contained within the gauge-boson part of the Lagrangian.

$$
\mathcal{L}_{W}=-\frac{1}{4} W_{i \mu \nu} W_{i}^{\mu \nu}-\frac{1}{4} B_{\mu \nu} B \mu \nu
$$

where: $W_{i \mu \nu}=\partial_{\mu} W_{i \nu}-\partial_{\nu} W_{i \mu}-g f_{i j k} W_{j \mu} W_{k \nu}$ and $B_{\mu \nu}=\partial_{\mu} B_{\nu}-\partial_{\nu} B_{\mu}$. Where $f_{i j k}$ are the structure constants of the weak isospin group.

The $W^{ \pm}$bosons in the SM carry electric charge $(q= \pm e)$ and weak isospin $\left(I^{W}= \pm 1\right)$. The $\gamma$ and $Z^{0}$ boson are neutral $\left(q=0, I^{W}=0\right)$ and are their own anti-particles. It is evident from this part of the Lagrangian that at the tree level, the only allowed self-interactions between the gauge-fields are the interactions between the charged and neutral bosons. This means that 
the only non-zero trilinear gauge couplings allowed in the SM are $W W \gamma$ and $W W Z$.

If only the possible trilinear vertices between the neutral gauge-bosons: $Z Z Z, Z Z \gamma, Z \gamma \gamma$ and $\gamma \gamma \gamma$ are considered, it is found that the vertex $\gamma \gamma \gamma$ is forbidden due to electromagnetic gauge invariance and $Z Z Z$ is not within the reach of the energies and luminosities available at the Tevatron. Therefore the only neutral triple gauge boson couplings, that can be investigated at the Tevatron are $Z Z \gamma$ and $Z \gamma \gamma$.

\subsection{4 $W^{ \pm} \gamma$ and $Z^{0} \gamma$ production}

The electroweak leading order (LO) or tree-level diagrams for $Z^{0} \gamma$ and $W^{ \pm} \gamma$ production $^{2}$ in $p \bar{p}$ collisions are shown in Figure 2.1. These processes occur via initial state radiation, final state radiation, and for $W \gamma$ production, via the $W W \gamma$ vertex in the s-channel diagram.

For the $Z^{0} \gamma$ process occurring via initial state radiation, the dilepton invariant mass $(m(l, l))$ distribution will be peaked around the $Z^{0}$-pole. For the final state radiation, the three body invariant mass $(m(l, l, \gamma))$ distribution will be peaked about the $Z^{0}$-pole. It can not be determined exactly which diagram produces a particular event, but if the dilepton invariant mass distribution is plotted for all events, as shown in Figure 2.2, the initial state radiation can be seen to populate the region above $86 \mathrm{GeV} / c^{2}$, and the final state radiation below this value.

As mentioned in section 2.1.3, the $Z Z \gamma$ and $Z \gamma \gamma$ vertices vanish at tree

\footnotetext{
${ }^{2}$ Strictly speaking the $Z^{0} \gamma$ production includes the Drell-Yan continuum, $\gamma^{*} \gamma$, but for brevity's sake this thesis will just refer to $Z^{0} \gamma$.
} 

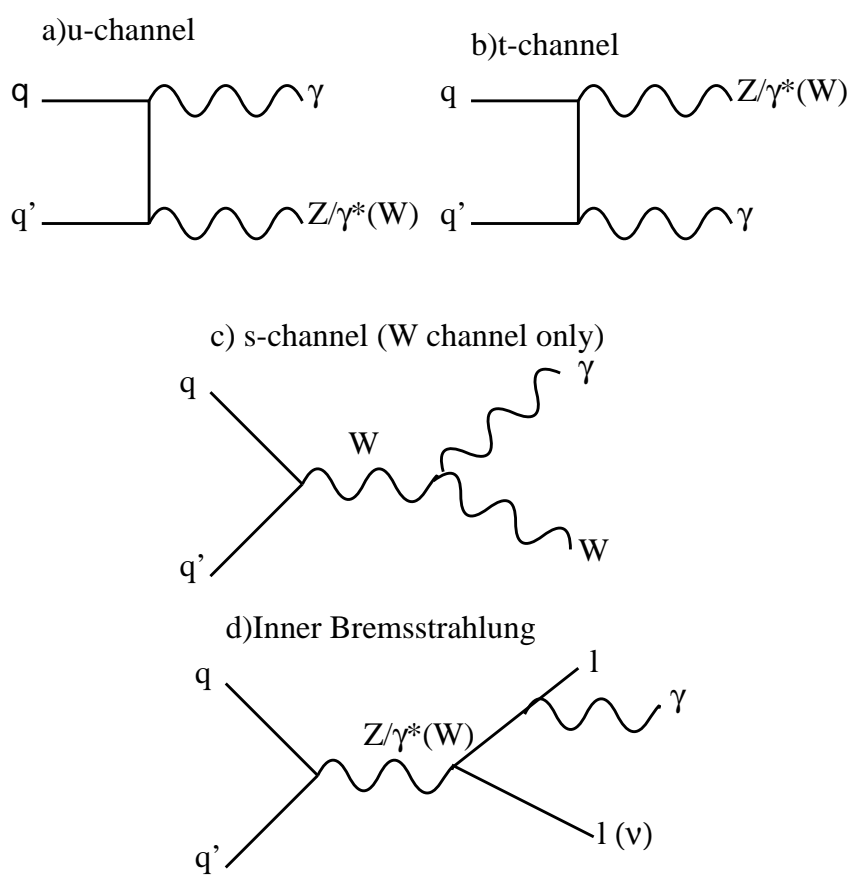

Figure 2.1: Tree-level diagrams for $Z^{0} \gamma$ and $W^{ \pm} \gamma$ production

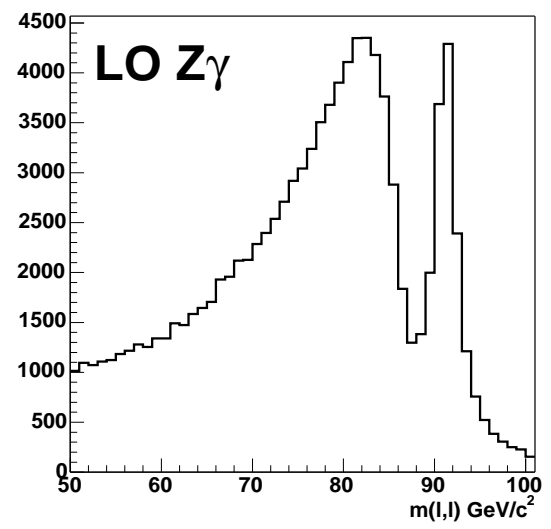

Figure 2.2: The generated invariant mass distributions for the leading order $Z^{0} \gamma$ processes produced from MC simulation. 
level in the Standard Model. However, in the presence of physics beyond that which is described in the SM, these vertices may be non-zero. An example of this would be if the $Z^{0}$ boson were in fact a composite particle, i,e, it had internal structure, or if excited $Z^{*}$ bosons existed which would decay into a $Z^{0}$ and a photon. Assuming electromagnetic gauge invariance and Lorentz invariance, a $Z V \gamma$ vertex (where $V=Z$ or $\gamma$ ) can be most generally described by four free parameters $h_{1}^{V}, h_{2}^{V}, h_{3}^{V}, h_{4}^{V}[10]$.

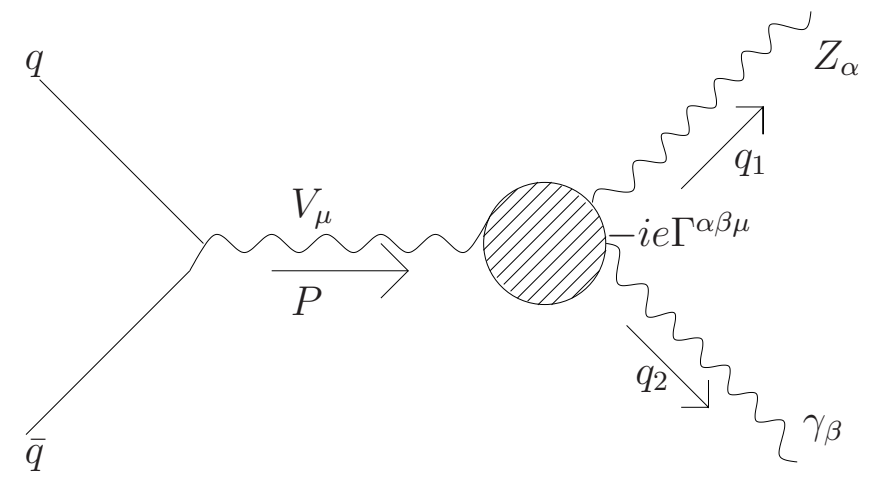

Figure 2.3: Trilinear $Z V \gamma$ vertex in $q \bar{q}$

Using the four-momentum notations from Figure 2.3, the vertex function for the $Z Z \gamma$ vertex as derived in [10] has the following form:

$$
\begin{aligned}
\Gamma_{\alpha \beta \mu}^{Z \gamma Z} & =\frac{P^{2}-q_{1}^{2}}{m_{Z}^{2}}\left(h_{1}^{Z}\left(q_{2}^{\mu} g^{\alpha \beta}-q_{2}^{\alpha} g^{\mu \beta}\right)\right. \\
& +\frac{h_{2}^{Z}}{m_{Z}^{2}} P^{\alpha}\left(\left(P \cdot q_{2}\right) g^{\mu \beta}-q_{2}^{\mu} P^{\beta}\right) \\
& \left.+h_{3}^{Z} \epsilon^{\mu \alpha \beta \rho} P_{\rho} q_{2 \sigma}+\frac{h_{4}^{Z}}{m_{Z}^{2}} P^{\alpha} \epsilon^{\mu \beta \rho \sigma} P_{\rho} q_{2 \sigma}\right)
\end{aligned}
$$

where $\epsilon^{\mu \alpha \beta \rho}$ is a totally antisymmetric tensor, and $m_{Z}$ is the mass of the $\mathrm{Z}$ 
boson. Similarly, the $Z \gamma \gamma$ vertex is given by:

$$
\begin{aligned}
\Gamma_{\alpha \beta \mu}^{Z \gamma \gamma} & =\frac{P^{2}}{m_{Z}^{2}}\left(h_{1}^{\gamma}\left(q_{2}^{\mu} g^{\alpha \beta}-q_{2}^{\alpha} g^{\mu \beta}\right)\right. \\
& +\frac{h_{2}^{\gamma}}{m_{Z}^{2}} P^{\alpha}\left(\left(P \cdot q_{2}\right) g^{\mu \beta}-q_{2}^{\mu} P^{\beta}\right) \\
& \left.+h_{3}^{\gamma} \epsilon^{\mu \alpha \beta \rho} P_{\rho} q_{2 \sigma}+\frac{h_{4}^{\gamma}}{m_{Z}^{2}} P^{\alpha} \epsilon^{\mu \beta \rho \sigma} P_{\rho} q_{2 \sigma}\right)
\end{aligned}
$$

Terms proportional to $P^{\mu}$ and $q_{1}^{\alpha}$ are omitted from equations since they do not contribute to the cross-section. Combinations of the $h_{3}^{V}\left(h_{1}^{V}\right)$ and $h_{4}^{V}\left(h_{2}^{V}\right)$ correspond to the electric (magnetic) dipole and magnetic (electric) quadrupole transition moment of the $\mathrm{Z}$ boson[15]. $Z Z \gamma$ and $Z \gamma \gamma$ couplings are restricted to their SM values of zero at asymptotically high energies by tree-level unitarity requirement. Generalised dipole form factors $\left(h_{i}^{V}=\right.$ $\left.h_{i}^{V}\left(m_{Z}^{2}, 0, \hat{s}\right)\right)$ are used to prevent unitarity violation:

$$
h_{i}^{V}\left(m_{Z}^{2}, 0, \hat{s}\right)=\frac{h_{i 0}^{V}}{\left(1+\hat{s} / \Lambda^{2}\right)^{n}} .
$$

This choice of form factor allows $h_{i}^{V}\left(m_{Z}^{2}, 0, \hat{s}\right)$ to tend to zero when $q_{1}^{2}, q_{2}^{2}$ or $P^{2}$ gets large. All the parameters, $h_{i}^{V}$, are C-odd dimensionless functions of $q_{1}^{2}, q_{2}^{2}$ and $P^{2}$. The parameters $h_{1}^{V}$ and $h_{2}^{V}$ are $P$-even, where as $h_{3}^{V}$ and $h_{4}^{V}$ are $P$-odd. This means that $h_{1}^{V}$ and $h_{2}^{V}$ are $C P$ violating terms where as $h_{3}^{V}$ and $h_{4}^{V}$ are $C P$ conserving.

Currently, the best limits on the $Z Z \gamma$ and $Z \gamma \gamma$ anomalous couplings are placed by the LEP experiments. Table 2.4 shows the limits from a twoparameter analysis [6]. Table 2.5 shows the current limits from a singleparameter analysis at D0, quoted for a form factor scale of $\Lambda=750 \mathrm{GeV}$.

Any non-zero value of the $h_{i}^{V}$ terms, would result in the inclusion of an s-channel diagram into the cross-section calculation for $Z^{0} \gamma$ production. This 


\begin{tabular}{|c|c|c|c|c|}
\hline Parameter & \multicolumn{2}{|c|}{$95 \%$ C.L. } & \multicolumn{2}{|c|}{ Correlations } \\
\hline$h_{1}^{\gamma}$ & {$[-0.16$} & $+0.05]$ & 1.00 & +0.79 \\
\hline$h_{2}^{\gamma}$ & {$[-0.11$} & $+0.02]$ & +0.79 & 1.00 \\
\hline$h_{3}^{\gamma}$ & {$[-0.08$} & $+0.14]$ & 1.00 & +0.97 \\
\hline$h_{4}^{\gamma}$ & {$[-0.04$} & $+0.11]$ & +0.97 & 1.00 \\
\hline$h_{1}^{Z}$ & {$[-0.35$} & $+0.28]$ & 1.00 & +0.79 \\
\hline$h_{2}^{Z}$ & {$[-0.21$} & $+0.17]$ & +0.79 & 1.00 \\
\hline$h_{3}^{Z}$ & {$[-0.37$} & $+0.29]$ & 1.00 & +0.76 \\
\hline$h_{4}^{Z}$ & {$[-0.19$} & $+0.21]$ & +0.76 & 1.00 \\
\hline
\end{tabular}

Table 2.4: The $95 \%$ C.L. intervals obtained combining the results from ALEPH, DELPHI and L3. In each case the two parameters listed are varied while the remaining ones are fixed to the SM values. Both the statistical and systematic uncertainties are included.[6]

\begin{tabular}{|lc|}
\hline Parameter & $95 \%$ C.L. \\
\hline$\left|h_{3}^{\gamma}\right|$ & $<0.37$ \\
$\left|h_{4}^{\gamma}\right|$ & $<0.05$ \\
$\left|h_{3}^{Z}\right|$ & $<0.36$ \\
$\left|h_{4}^{Z}\right|$ & $<0.05$ \\
\hline
\end{tabular}

Table 2.5: The $95 \%$ C.L. intervals obtained combining the results from D0, for a form factor scale $\Lambda=750 \mathrm{GeV}$. In each case, only one coupling is allowed to be non-zero. Both the statistical and systematic uncertainties are included.[16] 
would result in an increase in the cross-section above values predicted by the Standard Model.

\subsection{Monte Carlo (MC) Simulation}

A prediction of the number of $Z^{0} \gamma$ events expected at CDF is obtained using Monte Carlo simulation. A leading order (LO) matrix generator, ZGAMMA[21], is coupled to PYTHIA[81] to provide simulation of gluon radiation, hadronisation and the underlying event. After this, the events can be passed through a simulation of the CDF detector. In order to obtain a Next to Leading Order prediction (NLO), a correction factor known as a " $k$-factor" is applied to the LO simulation.

A description of the leading-order matrix element generator used is given in section 2.2.1. The " $k$-factor" used to obtain the NLO prediction is described in section 2.2.2. A summary of the uncertainties of the SM Expectation are given in section 2.2.3 and the NLO cross-section is given in section 2.2.4. A brief description of other MC samples used in this analysis is given in section 2.2.5.

\subsubsection{Leading-Order Matrix Element Generators}

The Feynman diagrams contributing to the $Z^{0} \gamma$ and $W^{ \pm} \gamma$ processes at the tree level are shown in Figure 2.1. Figures 2.1.a and 2.1.b show photon radiation from the incoming quarks in a process known as initial-state radiation. Diagram 2.1.c shows the triple gauge coupling vertex. This process is not allowed in the Standard Model in the $Z^{0} \gamma$ channel. Finally, Figure 2.1.d 
shows final state radiation (or inner bremsstrahlung) from the lepton. All of these diagrams must be included in any calculation that preserves gauge invariance.

The Monte Carlo matrix element generator ZGAMMA is provided by U. Baur and E. L. Berger [21]. The event generation uses a Monte Carlo integration program called VEGAS [17] and the tree-level calculation is performed using electroweak helicity amplitudes for $Z^{0} \gamma$ production and radiative $Z^{0}$ boson decays, as shown in figure 2.1.d. All interference terms are included.

Within the $Z^{0} \gamma$ calculation, there are divergences and singularities that must be avoided. The singularity which exists when the photon is emitted collinearly to a lepton is avoided in the ZGAMMA calculation by imposing a minimum lepton-photon angular separation $\Delta R_{l \gamma}$ :

$$
\Delta R_{l \gamma}=\left[\left(\Delta \phi_{l \gamma}\right)^{2}+\left(\Delta \eta_{l \gamma}\right)^{2}\right]^{1 / 2}
$$

where $\Delta \eta_{l \gamma}\left(\Delta \phi_{l \gamma}\right)$ is the difference in pseudo-rapidity (azimuthal angle) between the lepton and photon. These terms are explained in detail in section 3.3.1.

Infrared divergences are avoided, when generating the MC samples, by imposing a minimum cut on the transverse energy of the photon, $E_{T}^{\gamma}$, for the generated photons. Finally, a divergence exists as the invariant mass of the dilepton pair goes to zero (i.e. $m(l, l) \rightarrow 0)$. $Z^{0} \gamma$ events are generated using the ZGAMMA generator with kinematic cuts of $E_{T}^{\gamma}>5 \mathrm{GeV}, \Delta R_{l \gamma}>0.2$ and $m(l, l)>20$.

The ZGAMMA event generator does not include any QCD corrections to the $Z^{0}$ production. In order to remedy this, the matrix element generators are interfaced to PYTHIA (version 6.203) to provide initial state gluon showers and 
hadronisation as well as a simulation of the underlying event. An advantage of the ZGAMMA program, is that it includes mechanisms for the introduction of anomalous coupling terms.

The input parameters used are given in table 2.6 and are those recommended by the author [14]. A summary of all kinematic cuts on the generated particles used for the final MC $Z^{0} \gamma$ samples is given in table 2.7.

To provide confidence in the performance of the ZGAMMA event generator, comparisons were made with another event generator, MADGRAPH[19]. Table 2.8 shows how the predicted $Z^{0} \gamma$ cross-section compares for the ZGAMMA and MADGRAPH generators for different phase space regions. The equivalent program for $W \gamma$ production, WGAMMA[21], has already been found to have excellent agreement with the COMPHEP[18] event generator[12].

\subsection{2 k-factor}

Higher order QCD corrections shown in Figure 2.4, of the order- $\alpha_{s}$, increase

the cross-section of $Z^{0} \gamma$ production. To take these into account, a NLO correction factor known as a " $k$ " is determined. This $k$-factor is the ratio of the cross-section at NLO to that at LO, i.e.:

$$
k=\frac{\sigma_{N L O}}{\sigma_{L O}} .
$$

In order to obtain $k$, the NLO ZGAMMA program [22] is used to calculate $Z^{0} \gamma$ production at the Tevatron.

Unlike the LO ZGAMMA program, the NLO program only contains the initial state radiation channels. It does not contain any final state radiation diagrams. As discussed in section 2.1.4, a dilepton invariant mass cut of $86 \mathrm{GeV} / c^{2}$ can be used to distinguish the processes $p \bar{p} \rightarrow Z^{0} \rightarrow l^{+} l^{-} \gamma$ and 


\begin{tabular}{|l|c|}
\hline $\begin{array}{l}\text { Beam type } \\
\sqrt{s}[\mathrm{TeV}]\end{array}$ & 1.96 \\
\hline $\mathrm{PDF}$ & CTEQ5L \\
$Q_{f}^{2}$ & parton collision $\hat{s}$ \\
$\alpha_{s}\left(m_{Z}\right)$ & 0.127 \\
\hline$m_{W}[\mathrm{GeV}]$ & $80.41,79.97$ \\
$m_{Z}[\mathrm{GeV}]$ & $91.695,91.1884$ \\
$G_{F}[\mathrm{GeV}-2]$ & $1.6639 \times 10^{-5}$ \\
$\sin ^{2} \theta_{w}$ & 0.231 \\
$\alpha_{e m}$ & $1 / 127.51$ \\
$\Gamma_{W}[\mathrm{GeV}]$ & 2.103 \\
$\Gamma_{Z}[\mathrm{GeV}]$ & 2.514 \\
\hline
\end{tabular}

Table 2.6: Parameters used in ZGAMMA and WGAMMA

\begin{tabular}{|l|c|}
\hline$\left|\eta_{\gamma}\right|$ & $<10.0$ \\
$\left|\eta_{l e p}\right|$ & $<10.0$ \\
$\left|\eta_{\gamma}\right|$ & $<10.0$ \\
$E_{T}^{\gamma}$ & $>5.0 \mathrm{GeV}$ \\
$E_{T}^{l e p}$ & $>0.0 \mathrm{GeV}$ \\
$\Delta R_{l \gamma}$ & $>0.2$ \\
$m(l, l)$ & $>20.0 \mathrm{GeV} / c^{2}$ \\
$m(l, l, \gamma)$ & $>20.0 \mathrm{GeV} / c^{2}$ \\
\hline
\end{tabular}

Table 2.7: Parton level cuts used for final $Z^{0} \gamma$ samples 

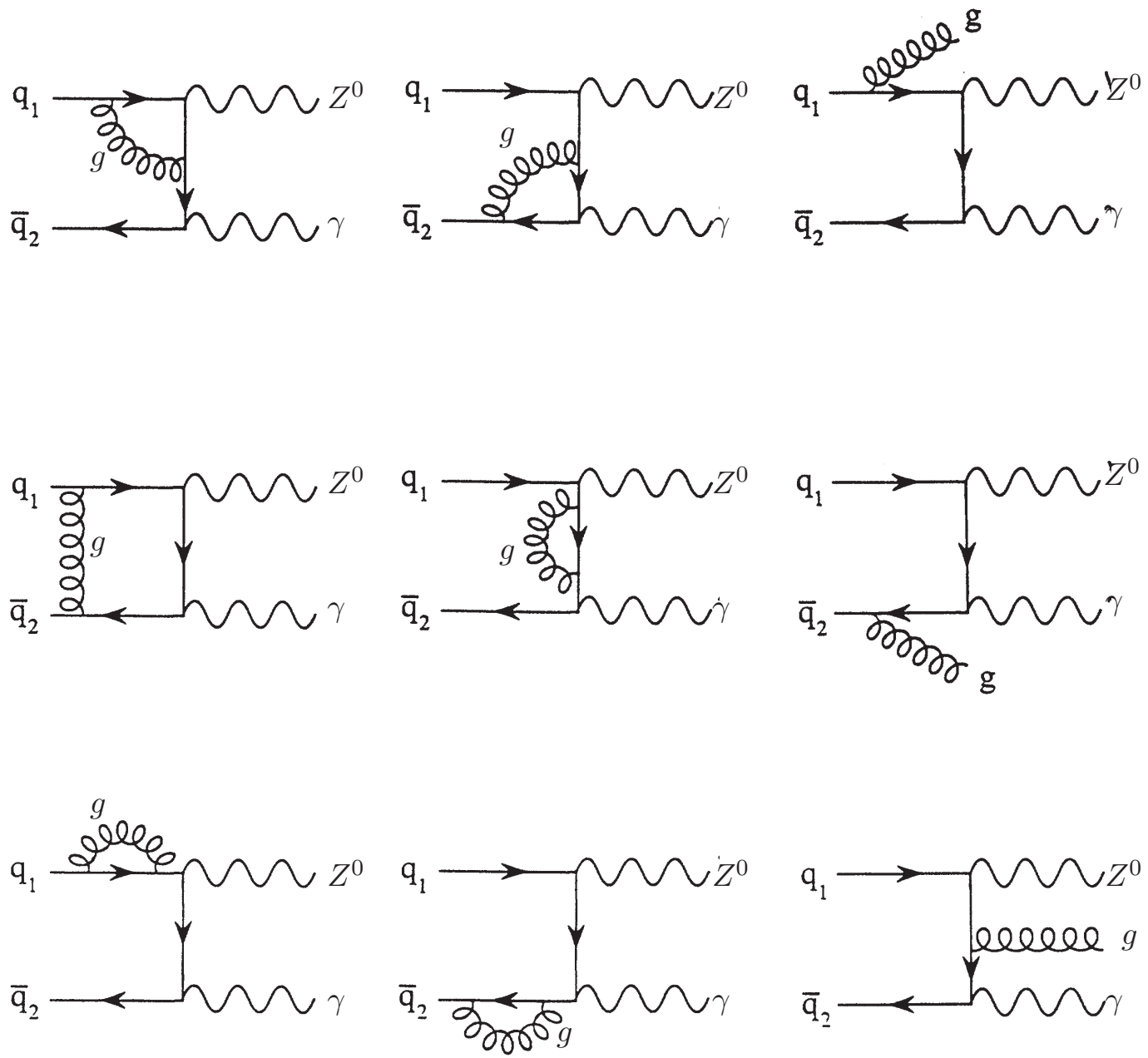

Figure 2.4: Virtual and soft gluon radiation, QCD corrections to the initial state radiation $Z^{0} \gamma$ processes. Not shown are the diagrams obtained by interchanging the $Z^{0}$ and the $\gamma \cdot[11]$ 


\begin{tabular}{|l|c|c|}
\hline & \multicolumn{2}{|c|}{$\sigma \times B R(\mathrm{pb})$} \\
& ZGAMMA & MADGRAPH \\
\hline$m(l l)>20 \mathrm{GeV} / c^{2}, E_{T}^{\gamma}>7 \mathrm{GeV}, \Delta R_{l \gamma}>0.7$ & $5.33 \pm 0.02$ & $5.32 \pm 0.01$ \\
$m(l l)>20 \mathrm{GeV} / c^{2}, E_{T}^{\gamma}>5 \mathrm{GeV}, \Delta R_{l \gamma}>0.2$ & $11.55 \pm 0.04$ & $11.44 \pm 0.03$ \\
$m(l l)>20 \mathrm{GeV} / c^{2}, E_{T}^{\gamma}>25 \mathrm{GeV}, \Delta R_{l \gamma}>0.7$ & $0.89 \pm 0.01$ & $0.90 \pm 0.01$ \\
$m(l l)>40 \mathrm{GeV} / c^{2}, E_{T}^{\gamma}>7 \mathrm{GeV}, \Delta R_{l \gamma}>0.7$ & $4.47 \pm 0.01$ & $4.46 \pm 0.01$ \\
\hline
\end{tabular}

Table 2.8: Comparison between ZGAMMA and MADGRAPH. A flat "k"-factor of 1.365 has been applied to all samples (see section 2.2.2).

$p \bar{p} \rightarrow Z^{0} \gamma \rightarrow l^{+} l^{-} \gamma$. Different corrections need to be applied to these two subsets.

- $p \bar{p} \rightarrow Z^{0} \rightarrow l^{+} l^{-} \gamma$

Events with $m(l, l)<86 \mathrm{GeV} / c^{2}$ are weighted with the inclusive $Z^{0}$ NLO correction of $1.36[13]$.

- $p \bar{p} \rightarrow Z^{0} \gamma \rightarrow l^{+} l^{-} \gamma$

Events with $m(l, l)>86 \mathrm{GeV} / c^{2}$ are weighted with an $E_{T}^{\gamma}$ dependent $k$-factor correction, calculated by taking the ratio of the NLO crosssection to that of the corresponding LO cross-section ${ }^{3}$. The Born crosssection, calculated by the NLO program, was made with CTEQ5L[23] using LO PDFs, while the NLO calculation was made using NLO PDFs with CTEQ5M[23].

\footnotetext{
${ }^{3}$ For this comparison, it is of critical importance to use appropriate PDFs for a meaningful correction. Ie, LO PDFs must be used for LO calculations and NLO PDFs must be used for NLO calculations. The program calculates the cross-section for the $\mathrm{u}$ and $\mathrm{t}$ processes at both LO and NLO.
} 
Figure 2.5 shows the ratio of the two cross-sections for $Z^{0} \gamma$ versus $E_{T}^{\gamma}$. The k-factor is calculated by the fit of this plot:

$$
k\left(Z^{0} \gamma, E_{T}^{\gamma}\right)=1.46+0.00073 \cdot E_{T}^{\gamma}-0.125 \cdot e^{-0.062 \cdot E_{T}^{\gamma}}
$$

The average $k$-factor correction integrated over all generated events with $E_{T}^{\gamma}>7 \mathrm{GeV}, \Delta R_{l \gamma}>0.7$ and $m(l, l)>40 \mathrm{GeV}$ is 1.365 for $Z^{0} \gamma$.

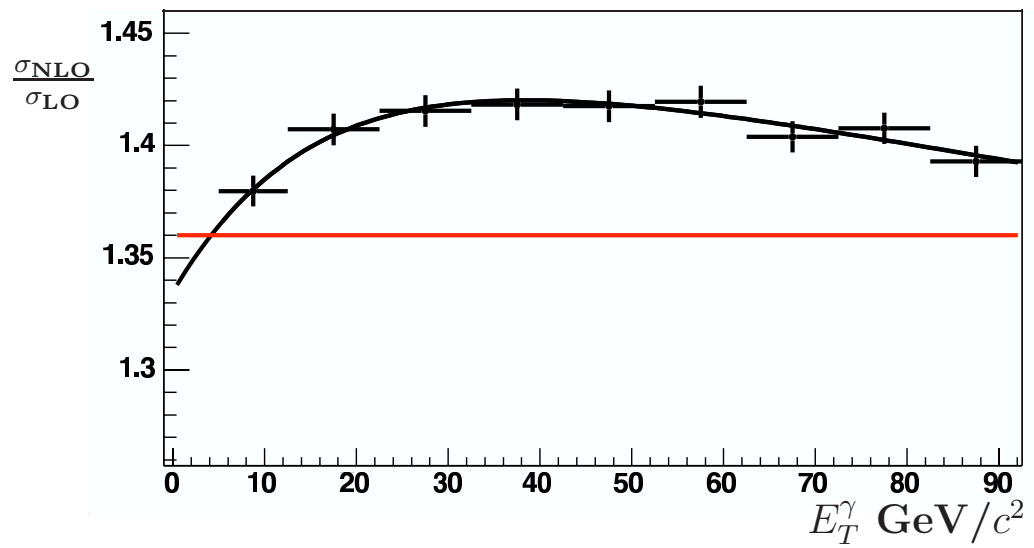

Figure 2.5: The k-factor correction for $Z^{0} \gamma$. This function is applied to events with $m(l, l)>86 \mathrm{GeV} / c^{2}$. The inclusive $Z^{0}$ NLO correction of 1.36 (shown as straight line), is applied to the inner bremstrahlung events.

\subsubsection{Uncertainty of SM Expectation}

Limitations in the theoretical precision of the calculation, result in an uncertainty on the cross-section prediction. The effect of these errors on the cross-section is summarised in table 2.9. 


\begin{tabular}{|l|c|}
\hline & $\delta \sigma$ \\
\hline Factorisation Scale & $2 \%$ \\
Unweight & 0 \\
PDF & $6 \%$ \\
K-Factor & $3 \%$ \\
\hline Total & $7 \%$ \\
\hline
\end{tabular}

Table 2.9: Systematic errors on the $Z^{0} \gamma$ MC generation

\section{Factorisation Scale}

The factorisation scale is the minimum $Q^{2}$ value calculated for photon emission in the ZGAMMA and WGAMMA programs. This value will affect the maximum $Q^{2}$ value for post generation Pythia fragmentation. The default factorisation scale was $\hat{s}$, the square of the collision energy of the event. The cross-section and acceptance were measured using four other values, $2 \hat{s}, 3 / 2 \hat{s}, 2 / 3 \hat{s}$ and $1 / 2 \hat{s}$. The greatest variation in the cross-section from the default value of $Q^{2}$ was $2 \%$.

\section{Parton Density Function choice}

Protons and anti-protons are composite particles. Therefore, any interactions between them must be described using parton density functions (PDF). The PDF describes the energy distributions of the valence quarks, gluons and sea quarks inside the proton/anti-proton.

The PDF chosen for use with ZGAMMA was the CTEQ5L PDF[23]. In order to determine the systematic error from this choice, the LO cross-section is compared to the corresponding predictions calculated from the MRST 72 
- 76 PDFs [24]. The MRST cross-sections range between 1.604 and 1.625 $\mathrm{pb}^{-1}$ whereas the cross-section using CTEQ5L is $1.72 \mathrm{pb}^{-1}$. The difference between the two was taken to be the systematic error, of $6 \%$.

\section{K-factor}

The calculated k-factor only takes into account $O\left(\alpha_{s}\right)$ corrections. To take into account higher order corrections, the $Q$ scale in the NLO calculation was varied by factors of 2 and 1/2. A 3\% variation in the cross-section calculation was observed, and taken to be a systematic error.

\subsubsection{Cross-section prediction}

Using the NLO correction derived in section 2.2.2, the calculated cross-section for $Z^{0} \gamma$ production in the kinematic range $m(l l)>40 \mathrm{GeV} / c^{2}, E_{T}^{\gamma}>7$ $\mathrm{GeV}, \Delta R_{l \gamma}>0.7$ is $4.5 \pm 0.3 \mathrm{pb}$.

\subsection{5 $\quad Z^{0} \rightarrow l^{+} l^{-}$MC samples}

Leading order $Z^{0} \rightarrow e^{+} e^{-}$and $Z^{0} \rightarrow \mu^{+} \mu^{-}$PYTHIA MC samples [81] are used in this analysis (see sections 4 and 5). These samples are generated and reconstructed using the same detector simulation as the ZGAMMA sample. These samples will be used in sections 4 and 5, for a measurement of the inclusive $Z^{0}$ production cross-section, used as a cross check in this analysis. 


\section{Chapter 3}

\section{The CDF Experiment at the Tevatron Collider}

The Tevatron is currently the world's highest energy particle accelerator. It accelerates protons and antiprotons to energies of $0.98 \mathrm{TeV}$, which collide at two points. Two general purpose detectors are situated at these two collision points, the Collider Detector at Fermilab (CDF) and D0.

\subsection{A Little History}

The first collisions were produced in October 1985, and since then, the Tevatron and both detectors have evolved with increasing energy and luminosity to yield datasets summarised in table 3.1.

CDF collected $109 \mathrm{pb}^{-1}$, at a centre-of-mass energy of $1.8 \mathrm{TeV}$ in the period from 1992 to 1996 ("Run I"). Instantaneous luminosites in excess of $2 \times 10^{31} \mathrm{~cm}^{-2} \mathrm{~s}^{-1}$ were obtained. In 2001, "Run II" started. The Tevatron was upgraded to increase both the centre-of-mass energy, from 1.8 to $1.96 \mathrm{TeV}$, 


$$
\begin{array}{ll}
25 \mathrm{nb}^{-1} & \text { in } 1987 \\
4.5 \mathrm{pb}^{-1} & \text { in 1988-1989 (Run 0) } \\
19 \mathrm{pb}^{-1} & \text { in 1992-1993 (Run Ia) } \\
90 \mathrm{pb}^{-1} & \text { in 1994-1996 (Run Ib) } \\
400 \mathrm{pb}^{-1} & \text { in 2002-09/2004 (Run II so far) }
\end{array}
$$

Table 3.1: Luminosities obtained for different data collecting runs at the Tevatron.

and the instantaneous luminosity up to $2 \times 10^{32} \mathrm{~cm}^{-2} \mathrm{~s}^{-1}$. This increase in luminosity required upgrades in both detectors. At the current time (October 2004), the record instantaneous peak luminosity obtained for Run II is $1 \times$ $10^{32} \mathrm{~cm}^{-2} \mathrm{~s}^{-1}$ and a $\mathrm{L}=680 \mathrm{pb}^{-1}$ has been delivered.

The Fermilab accelerator complex is discussed in section 3.2 and details of the CDF detector are given in section 3.3.

\subsection{The Fermilab Accelerator Complex}

The Fermilab accelerator complex is shown schematically in Figure 3.1. The Tevatron is a circular proton synchrotron $1 \mathrm{~km}$ in radius, producing protonantiproton collisions with a centre-of-mass energy of $\sqrt{s}=1.96 \mathrm{TeV}$.

\subsubsection{Proton Acceleration}

The first step in the proton acceleration process begins with a pulsed ion source that converts hydrogen gas into $\mathrm{H}^{-}$ions. These ions are then injected into a Cockroft-Walton electrostatic accelerator where a series of voltage gaps 


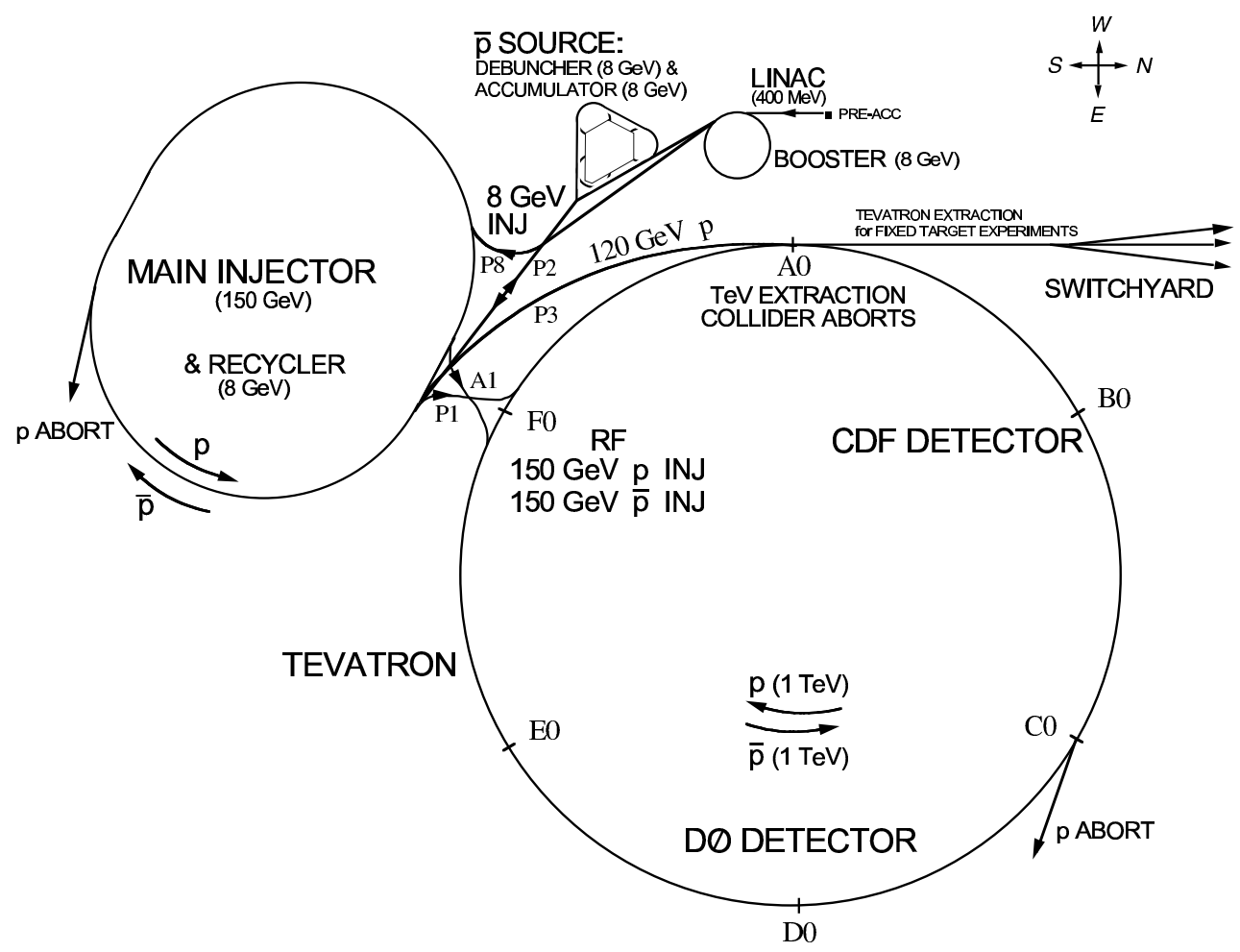

Figure 3.1: The Fermilab accelerator complex. 
accelerate them to an energy of $750 \mathrm{keV}$. The ions are then injected into a $145 \mathrm{~m}$ linear accelerator (the Linac) and accelerated to $400 \mathrm{MeV}$, by boosting them through a series of oscillating electric fields. Due to the nature of this oscillating field, the particles travelling through the Linac are arranged in pulses. A typical pulse contains 6 trillion particles[25].

After passing through carbon foil to remove the orbital electrons, the resulting protons are passed into the Booster ring. This ring is the first of six synchrotrons which act in a chain at Fermilab, culminating in the Tevatron. The Booster is $475 \mathrm{~m}$ in circumference and accelerates the protons from 400 $\mathrm{MeV}$ to $8 \mathrm{GeV}$ in a period of 0.033 seconds. The synchrotron uses powerful magnets to force the beam to travel in a circular orbit. The magnetic field must be increased as the protons are accelerated in order to maintain the correct orbit. Quadrupole magnets are used to focus the beam.

The Booster provides a number of separate regions of stable acceleration known as buckets. The protons are collected in these buckets to form bunches. The Booster transfers between six and eight bunches into the Main Injector at a time.

The Main Injector, two miles in circumference, serves a dual purpose. It accelerates the beam to $120 \mathrm{GeV}$ for fixed target experiments and to 150 $\mathrm{GeV}$ for injection into the Tevatron [26]. The six to eight bunches from the booster are combined into a single bunch consisting of approximately $6 \times 10^{13}$ protons. 


\subsubsection{Anti-proton Production}

Anti-protons are produced by focusing $120 \mathrm{GeV}$ protons, from the Main Injector, on to a nickel target every 1.5 seconds. Many different particles are produced with a wide range of angles and momenta. For every million protons which are collided only about 20 anti-protons are produced. They are collected and focused by a cylindrical lithium lens, $15 \mathrm{~cm}$ long and $2 \mathrm{~cm}$ in diameter. Pulsed dipole magnets act as a charge-mass spectrometer to select negatively charged anti-protons of approximately $8 \mathrm{GeV}$ in energy[27].

The $120 \mathrm{GeV}$ protons that arrive on the nickel target are bunched. This means that the anti-protons, although fewer in number, will also be bunched. They will also have a spread of energies. In order to reduce the spread of energies and to increase the spread in time, they are injected into a Debuncher ring. A process known as "stochastic cooling" [27] is used to reduce the transverse beam size and range of energies. The anti-protons are then stored in the Accumulator[27]. This device can store successive stacks of antiprotons for up-to several days or until they are required by the Main Injector for injection into the Tevatron.

When a stack of a suitable size is obtained in the Accumulator, the antiprotons are injected in to the Tevatron to be accelerated to $980 \mathrm{GeV}$. The protons and anti-protons, being of opposite charge, are accelerated along the beam pipe in opposite directions, held in two different orbits. Thirty-six bunches of protons and antiprotons, spaced by $396 \mathrm{~ns}$, are brought into collisions once the required energy is reached. The Tevatron is a super-conducting synchrotron, with about 1000 super-conducting magnets. These magnets must be cooled to $4^{\circ} \mathrm{K}$ in order to provide a magnetic field of $4.2 \mathrm{~T}$. After 
the two beams of particles have been accelerated to $0.98 \mathrm{TeV}$, quadrupole magnets installed at CDF and D0 reduce the transverse dimensions of the beams, and direct them towards the two collision points.

\subsection{The CDF Detector}

The CDF detector [30] is a general purpose solenoidal detector which combines precision particle tracking, calorimetry and muon detection. It has been designed to measure the position and momentum of charged and neutral particles over a large range of the solid angle. With this design it can be used to study a broad range of final states in $p \bar{p}$ collisions. The detector is symmetric around the beam axis in design. A cross-section of one half of the detector is shown in Figure 3.2.

\subsubsection{CDF Co-ordinate System and Related Nomenclature}

Each proton and anti-proton contains three valence quarks and a sea of softer quarks and gluons. A typical collision is actually an interaction of one of the valence quarks from the proton and one from the antiproton. It is impossible to know exactly what fraction of the incoming proton's momentum, the colliding quark (or anti-quark) has. This means that the centre of mass of the interaction may not occur in the lab frame. Two variables, which are invariant under Lorentz boosts along the beamline, are frequently used at hadron colliders. These are the $P_{T}$, the component of a momentum transverse to the beamline, and rapidity $(y)$. Rapidity is defined as $y=\frac{1}{2} \log \frac{E+P_{L}}{E-P_{L}}$ or 


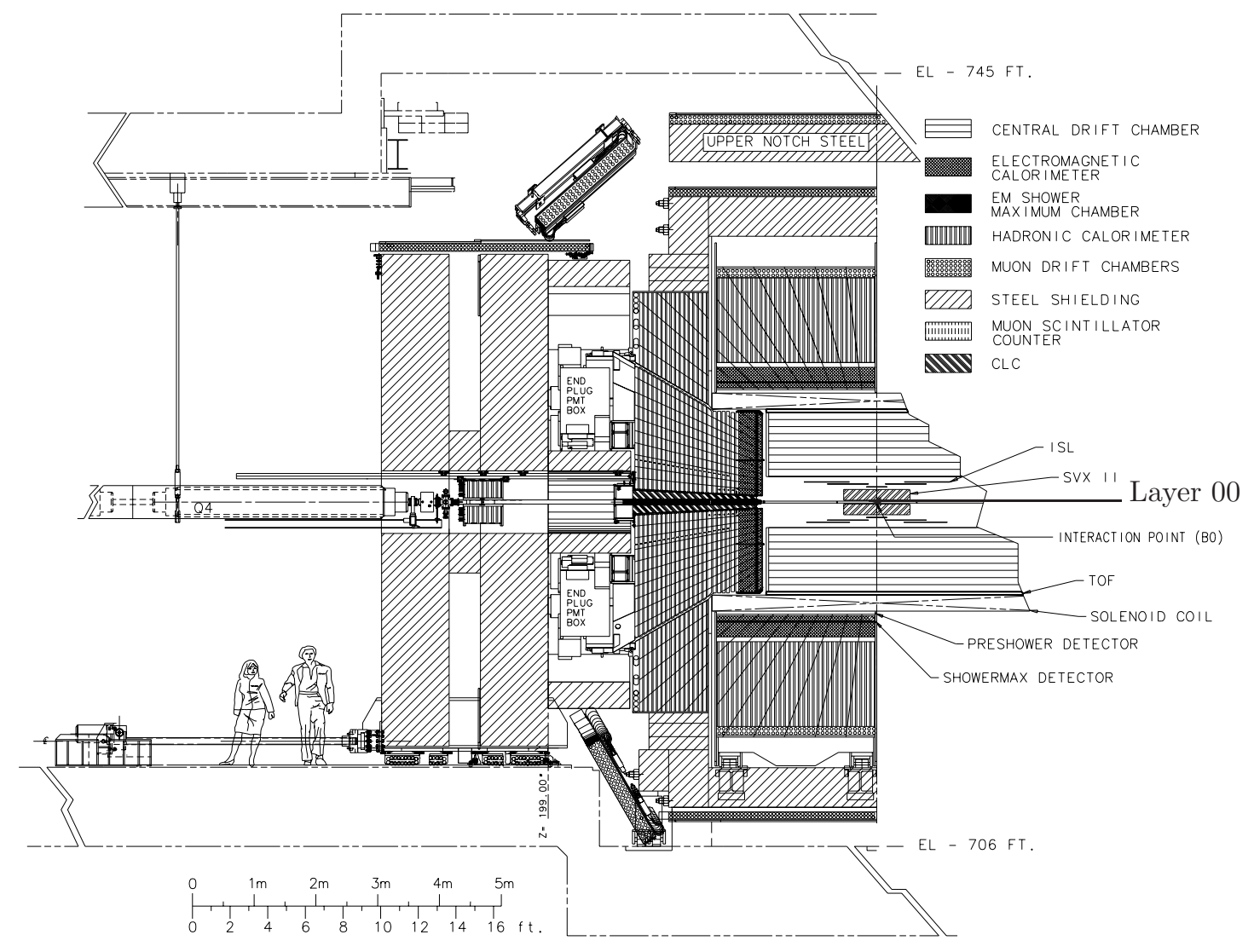

Figure 3.2: Cross-section view of CDF in the r-z view (see section 3.3.1). 
$\tanh (y)=\frac{P_{L}}{E}$, where $P_{L}$ is the longitudinal momentum along the beamline and $E$ is the energy.

A cylindrical coordinate system $(r, \phi, z)$ is used at CDF. The origin is taken to be the nominal interaction point at the centre of the detector shown in Figure 3.3. The axis of symmetry (z-axis) points in the direction of the proton beam and the azimuthal angle, $\phi$, is measured from the plane of the Tevatron. Spherical coordinates $(r, \phi, \theta)$ are also used. The polar angle, $\theta$ is measured from the proton direction. Another variable frequently used is the pseudo-rapidity, $\eta=-\ln \left(\tan \left(\frac{\theta}{2}\right)\right)$.

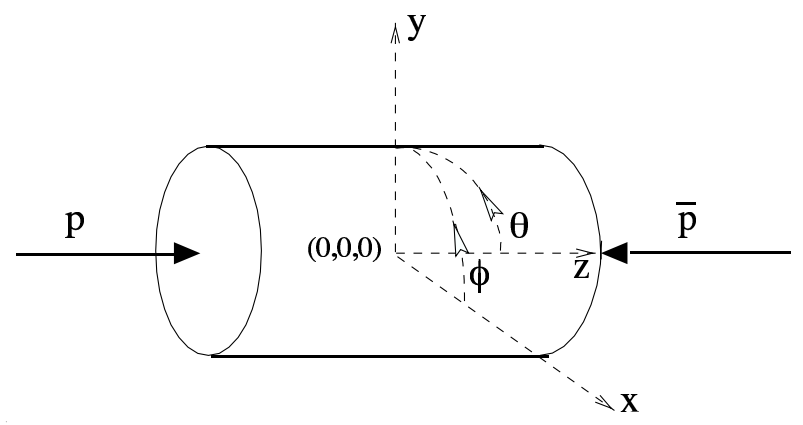

Figure 3.3: The CDF coordinate system.

\subsubsection{Tracking Systems}

The tracking detectors at CDF provide a measurement of both the position and momentum of charged particles produced in the interaction. A solenoid, $3 \mathrm{~m}$ in diameter and $5 \mathrm{~m}$ in length, provides a 1.4 Tesla magnetic field parallel to the beamline. Any charged particle travelling through the tracking systems will experience a Lorentz force, causing them to travel in a curved path, the curvature of which is determined by their momentum. 
The tracking system consists of silicon detectors and a central drift chamber. These are now described in detail. A summary of the design parameters are given in table 3.2 [34].

\section{Silicon Tracking Systems}

The CDF silicon tracking system is the sub-detector closest to the interaction region. The silicon tracking system consists of three subdetectors which provide tracking for the region $|\eta| \leq 2$.

The three detectors in ascending order of radius from the beamline are Layer 00 (L00) at a radius of $1.35 \mathrm{~cm}$, the Silicon Vertex Detector (SVX II) and the Intermediate Silicon Layers (ISL) detector. Layer 00 consists of single-sided, silicon strip detectors, details of which can be found in [31].

The Silicon Vertex Detector detector [32][33] consists of three identical cylindrical barrels with a total length of $96 \mathrm{~cm}$. Each barrel has 5 layers of double-sided silicon microstrip detectors between radii of 2.4 and $10.7 \mathrm{~cm}$. These layers are arranged into twelve wedges in $\phi$, as shown in Figure 3.4.

The Intermediate Silicon Layers (ISL) detector [32][33] is a large silicon tracker located between the SVX II and the COT. It consists of one central layer $(|\eta| \leq 1)$ at a radius of $23 \mathrm{~cm}$, and two forward-backward $(1 \leq|\eta| \leq 2)$ layers at radii of $20 \mathrm{~cm}$ and $29 \mathrm{~cm}$. A summary of the design parameters of both the SVX II and the ISL are given in table 3.2.

\section{Central Outer Tracker: COT}

The tracking at large radii in the central region, $|\eta| \leq 1.0$, is achieved by a large open cell drift chamber known as the Central Outer Tracker (COT)[35]. 


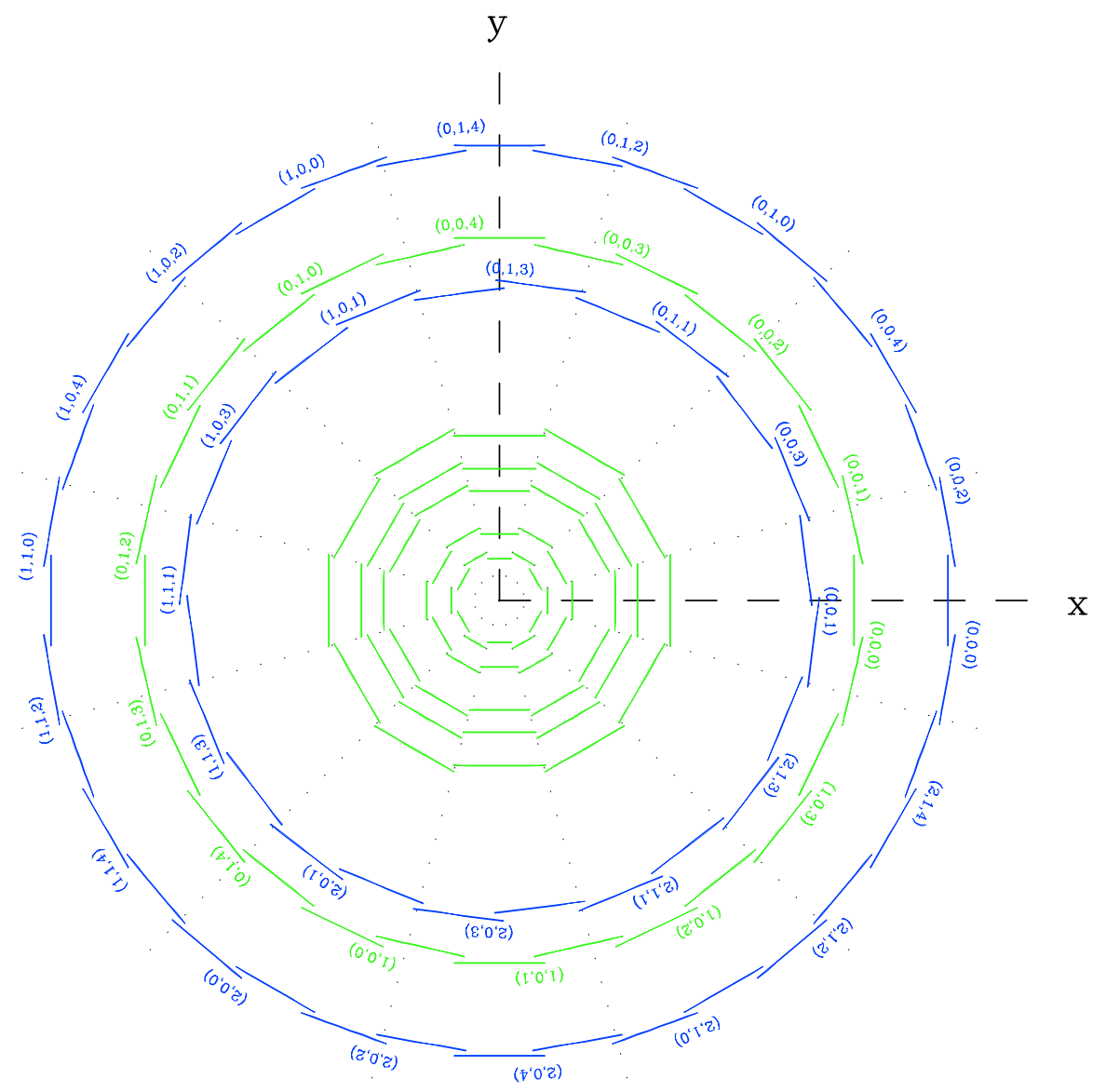

Figure 3.4: The cross-section of SVXII and ISL tracking systems. 
It is positioned outside the silicon tracking detectors and inside the solenoid. The COT has 30,240 sense wires which run along its length (in z), between two end plates $310 \mathrm{~cm}$ apart. Approximately half these wires run along the $\mathrm{z}$ direction (axial) and the other half (stereo) are aligned at an angle of $2^{\circ}$ with respect to the axial wires. The wires are arranged in 8 so-called superlayers, each containing 12 wire planes, as shown in Figure 3.5.

The COT has been designed to limit drift times to less than 100 ns. To achieve this small drift cells and a fast gas mixture (50:50 Ar-Et) are used.

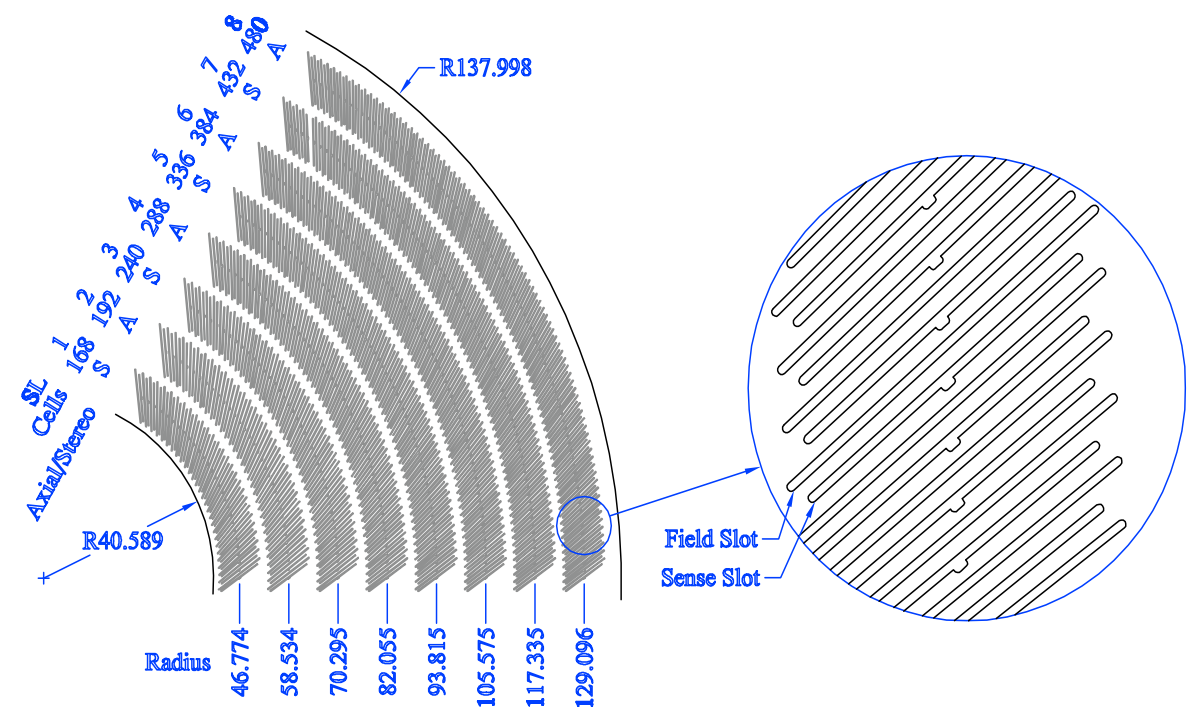

Figure 3.5: Schematic diagram of the arrangement of the 8 axial and stereo superlayers comprising the Central Outer Tracker.

The basic drift cell consists of two gold-on-mylar cathode planes, separated by $1.76 \mathrm{~cm}$, that form the walls of the drift cell. Down the middle is a line of alternating sense and potential wires every $3.8 \mathrm{~mm}$, as shown in Figure 3.6. 
+ Potential wires

- Sense wires

$\times$ Shaper wires

- Bare Mylar

— Gold on Mylar (Field Panel)

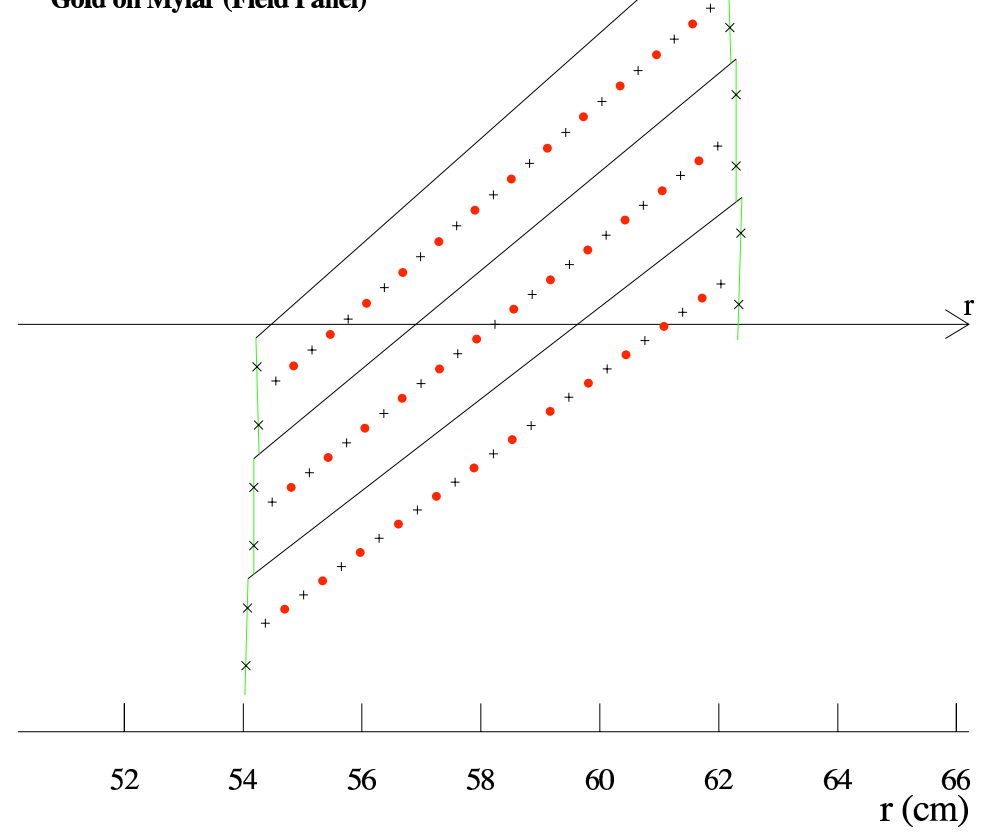

Figure 3.6: An example of a COT drift cell in Superlayer 2 


\begin{tabular}{|c|c|}
\hline SVX II & \\
\hline Radial coverage & 2.4 to $10.7 \mathrm{~cm}$, staggered quadrants \\
\hline Number of layers & 5 \\
\hline Readout coordinates & $r-\phi$ on one side of all layers \\
\hline Stereo side & $\mathrm{r}-\mathrm{z}, \mathrm{r}-\mathrm{z}, \mathrm{r}-\mathrm{uv}, \mathrm{r}-\mathrm{z}, \mathrm{r}-\mathrm{uv}\left(\mathrm{uv} \equiv 1.2^{\circ}\right.$ stereo $)$ \\
\hline Resolution per measurement & $12 \mu \mathrm{m}$ (axial) \\
\hline Total length & $96.0 \mathrm{~cm}$ \\
\hline Rapidity coverage & $|\eta| \leq 2.0$ \\
\hline Number of channels & 405,504 \\
\hline Material thickness & $3.5 \% X_{0}$ \\
\hline \multicolumn{2}{|l|}{ ISL } \\
\hline Radial coverage & 20 to $28 \mathrm{~cm}$ \\
\hline Number of layers & one for $|\eta| \leq 1 ;$ two for $1<|\eta|<2$ \\
\hline Resolution per measurement & $16 \mu \mathrm{m}$ (stereo) \\
\hline Total length & $174 \mathrm{~cm}$ \\
\hline Rapidity coverage & $|\eta| \leq 1.9$ \\
\hline Number of channels & 268,800 \\
\hline Material thickness & $2 \% X_{0}$ \\
\hline \multicolumn{2}{|l|}{$\mathrm{COT}$} \\
\hline Radial coverage & 44 to $132 \mathrm{~cm}$ \\
\hline Number of superlayers & 8 \\
\hline Number of wires per superlayer & 12 \\
\hline Maximum drift distance & $0.88 \mathrm{~cm}$ \\
\hline Resolution per measurement & $180 \mu \mathrm{m}$ \\
\hline Rapidity coverage & $|\eta| \leq 1.0$ \\
\hline Number of channels & 30,420 \\
\hline Material thickness & $1.3 \% X_{0}$ \\
\hline
\end{tabular}

Table 3.2: Design parameters of the tracking systems [30] 


\section{Tracking Algorithmss}

The tracking detectors are used together to provide a resolution of $30 \mu \mathrm{m}$ in both the $z$ and $r-\phi$ view, and a momentum resolution of $\sigma_{P_{T}} / P_{T}^{2}$ of $0.08 \%$ at $20 \mathrm{GeV}[30]$. Algorithms are used to reconstruct particle tracks from the hits in the tracking systems. There are four main tracking algorithms used at $\mathrm{CDF}$.

1. COT Pattern Recognition Algorithm [36]

Hits in individual axial and stereo superlayers are grouped together to find segments of tracks containing 3 or more hits in consecutive wires. Next $r-\phi$ tracks are found by linking together segments in the four axial superlayers. Segments or hits in the stereo layers can then be added to form 3D reconstructed tracks.

2. OI

In the central region, an "outside-in" (OI) tracking algorithm is used. COT tracks are extrapolated into the silicon detectors, adding hits progressively. Hits within $\pm 4 \sigma$ of the extrapolated track position, at each silicon layer are added to form new track candidates. The candidate with the greatest number of silicon hits is ultimately chosen [38]. For the range $|\eta|<1.1$, the efficiency of the OI algorithm in 99.6 $\pm 0.4 \%[40]$, falling rapidly to 0 beyond $|\eta|>1.2$ for isolated particles.

3. Standalone Silicon Tracking

This algorithm is not used for this analysis. An "inside-out" (IO) tracking algorithm is used [39].

4. PHOENIX Tracking 
In the plug region, an alternative tracking algorithm know as PHOENIX tracking exists to provide tracking for electrons [41, 42]. This algorithm uses the energy and position measurements from the plug calorimeter to provide a seed for the silicon tracking system. The efficiency of the PHOENIX algorithm is about $90 \%$ at $|\eta| \sim 1.2$, falling to $40 \%$ at $|\eta| \sim 2.6$ due to the eta coverage of the silicon.

In order to create the seed tracks, three pieces of information are required. Two position measurements are obtained from the assumed vertex of the electron, given by the primary vertex of the event, and the second from the most energetic shower cluster ${ }^{1}$. The third piece of information required is the curvature, given by the momentum of the electron. The absolute value of this can be given by the total $E_{T}$ of the energy cluster. As the charge is unknown, two seed tracks are created, one for each charge hypothesis. The OI tracking algorithm is then applied to both hypotheses. In the case that tracks are found for both charge hypotheses, a $\chi^{2}$ parameter describing the quality of the fit to the silicon hits can be used to discriminate, if required by the analysis.

Figure 3.7 shows the tracking efficiency of the OI, silicon stand-alone and the PHOENIX tracking algorithm.

\subsubsection{Time of Flight System}

The Time of Flight detector (TOF) [87] measures the time taken by a particle to travel from the interaction point to the detector, and has a particle timing

\footnotetext{
${ }^{1}$ See section 3.3 .4
} 


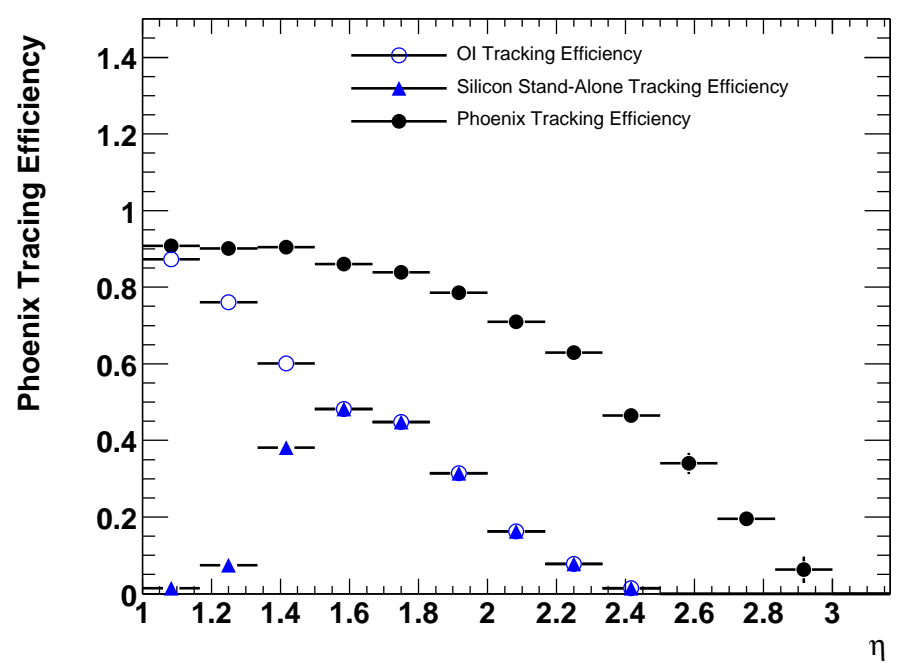

Figure 3.7: Tracking efficiencies of the different tracking algorithms.

resolution of 100 ps. This information can be used to help tag cosmic events. The TOF is situated between the COT and the solenoid. It consists of 216 scintillator bars with dimensions $4 \times 4 \times 276 \mathrm{~cm}$. A photomultiplier tube is mounted at each end of the scintillator bars.

\subsubsection{Calorimeter Systems}

The calorimeters are segmented into $15^{\circ}$ in the azimuth and 0.1 in pseudorapidity, to form projective towers pointing back to the nominal interaction point [30]. Each tower consists of an inner lead-scintillator electromagnetic (EM) section, backed up by a steel-scintillator central hadron calorimeter on the outside. They are separated into the central $(|\eta|<1.1)$ and plug $(1.1<|\eta|<3.6)$ regions. The region $0.77<\eta<1.0,75^{\circ}<\phi<90^{\circ}$ is uninstrumented to allow for cryogenic utilities servicing the solenoid. The central calorimeter systems have been retained from Run I, but the plug 
calorimeters are new detectors for Run II.

\section{Central Electromagnetic Calorimeter (CEM):}

Any high energy electron or photon, passing through the electromagnetic calorimeters, will undergo pair production $\left(\gamma \rightarrow e^{+} e^{-}\right)$and bremsstrahlung $\left(e^{ \pm} \rightarrow \gamma e^{ \pm}\right)$producing subsequent electrons and photons to continue these processes. In this manner an electromagnetic shower is produced. The point at which the electromagnetic shower consists of the largest amount of particles is known as the shower maximum. At this point the average energy per particle becomes low enough as to prevent further multiplication. After the shower maximum, the shower decays slowly through ionization losses for the electrons and positrons or by Compton scattering for the photons. The calorimeters measure the energy deposited by these showers, and hence the energy of the incident particle.

Showers from electrons and photons are typically contained within the CEM. Hadrons passing through the electromagnetic calorimeter can also produce a shower but most of their shower will develop in the hadronic calorimeter.

The CEM [43][30] provides coverage for $|\eta| \leq 1.1$, with an energy resolution of $\frac{13.7 \%}{\sqrt{E}} \oplus 1.5 \%$. The CEM consists of two physically separate halves ("East" and "West") 24 wedges in azimuth. Each CEM wedge consists of alternating layers of plastic scintillator, as a sampling medium, and a lead absorber. There are 31 layers of the scintillator, each $5 \mathrm{~mm}$ thick and 30 layers of the lead, 3mm thick. A typical CEM tower is shown schematically in Figure 3.8. The central calorimeter towers are 18 radiation lengths deep, 
and the active elements of the scintillator tiles are read out by wavelength shifting fibres that direct the light to photomultiplier tubes (PMT).

To provide more accurate information on the position of the electromagnetic shower inside the calorimeter, the Central Electromagnetic Shower (CES) [43] detector is embedded inside the CEM at the shower maximum, at a depth of approximately 6 radiation lengths. The CES detector is a proportional strip and wire chamber situated at a radius of $184 \mathrm{~cm}$ from the beamline. In the azimuth direction, cathode strips are used to provide the $\mathrm{z}$ position and in the $\phi$ direction, anode wires are used. These wires can effectively measure the transverse shower profile to distinguish between a single shower from a prompt photon and two showers from a decay of a neutral meson to two photons, e.g. $\pi^{0} \rightarrow \gamma \gamma$, with a position resolution of $2 \mathrm{~mm}$ at $50 \mathrm{GeV}$.

In order to help particle identification, specifically between electromagnetic and hadronic showers the central preradiator detector $(\mathrm{CPR})$ is mounted on the front of the calorimeter wedges, at a radius of $168 \mathrm{~cm}$ from the beamline, and uses the solenoid and tracking detectors as a radiator. It uses proportional chambers to sample the early development of the shower to measure conversions in the coil, helping to distinguish prompt photons and electrons from photons originating from $\pi^{0}$ decays and electrons from conversions. A prompt photon has a $60 \%$ probability of converting, while the conversion probability of at least one photon from $\pi^{0} \rightarrow \gamma \gamma$ is about $80 \%$ (see section 7.4.3 for details).

Each CPR chamber is $116 \mathrm{~cm}$ long, $37.3 \mathrm{~cm}$ wide and $2.85 \mathrm{~cm}$ deep. The chambers have $2.22 \mathrm{~cm}$ cells segmented in $r-\phi$. The chambers have 33 


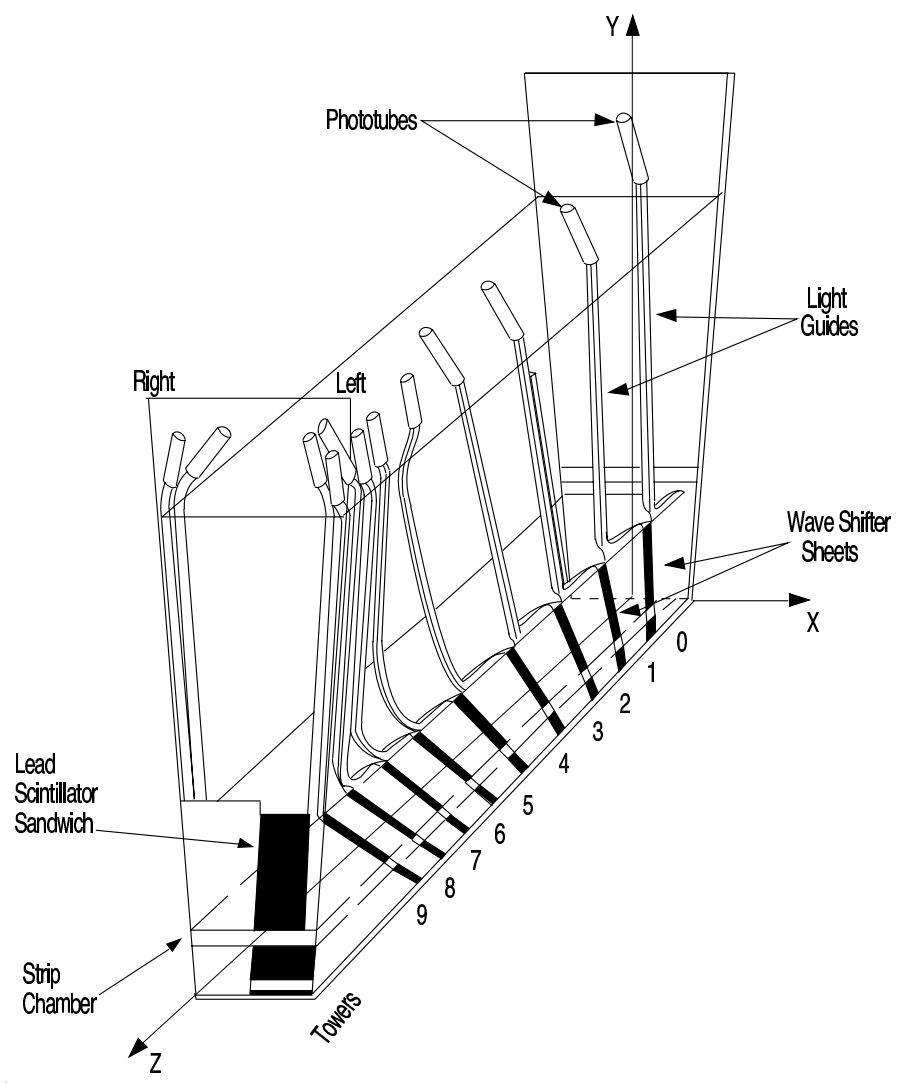

Figure 3.8: Schematic of a typical central electromagnetic calorimeter wedge. 
ground wires alternating with 32 sense wires, which are kept at $1790 \mathrm{~V}$. The wires are sandwiched between Cu-clad FR4 and 1/4" Hexcell as shown in Figure 3.9[45]. Further details are given in table 3.3.

\begin{tabular}{|l|c|}
\hline Total Depth & 1.125 in. \\
Total Width & 14.6875 in. \\
Total Length & 45.75 in. \\
Number of Grounded Field Wires/Chamber & 33 \\
Number of Sense Wires/Chamber & 32 \\
Wire Spacing & $7 / 32 \mathrm{in}$. \\
Cell Size with Ganging & $28 / 32 \mathrm{in}$. \\
Number of Readout Channels/Wedge & 32 \\
Chamber Gain from Fe 55 & $32 \mathrm{~K}$ \\
Source Capacitance & $960 \mathrm{pF}$ \\
\hline
\end{tabular}

Table 3.3: Description of the Central Preradiator Chambers [45]

\section{Central and Endwall Hadronic Calorimeter (CHA and WHA):}

A hadron shower is produced when an incident hadron undergoes an inelastic collision, producing secondary hadrons which can interact in a similar manner. Although hadron showers may start showering in the electromagnetic calorimeters, they will only be fully absorbed in the hadronic calorimeters due to their denser composition. Each wedge of the CEM is backed by a steel-scintillator central hadron calorimeter (CHA), which allows easy calculation of the ratio of electromagnetic to hadronic energy for each tower. This ratio is important in identification of electrons and photons. Each CHA 


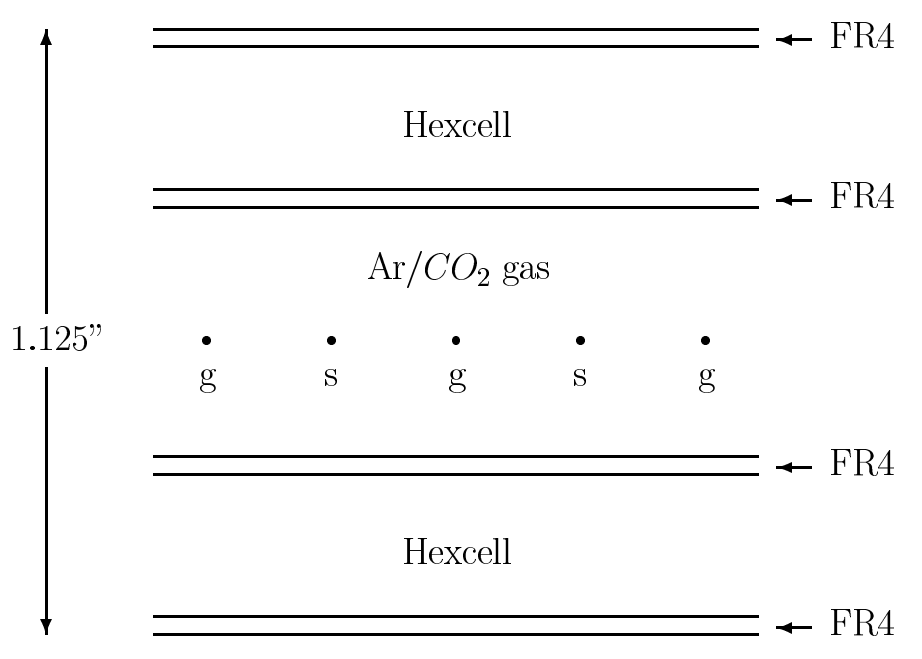

Figure 3.9: Schematic, head on view, of a CPR cell. Shown are the ground (g) and signal (s) wires.

wedge consists of 32 layers of a $5 \mathrm{~cm}$ thick steel between layers of a plastic scintillator, $1 \mathrm{~cm}$ thick. The energy resolution of the $\mathrm{CHA}$ and WHA are $50 \% / \sqrt{E_{T}} \oplus 3 \%$ and $75 \% / \sqrt{E_{T}} \oplus 4 \%$ respectively.

\section{Plug Region}

The plug calorimeter has been added for Run II. It provides coverage for the region $1.1<|\eta|<3.6$ and has a similar construction to the central calorimeter system. A cross-section of the top half of one plug is shown in Figure 3.10. Like the central calorimeter system, it consists of towers divided into an inner electromagnetic section (PEM) followed by an outer hadronic section (PHA), with scintillator tiles read out by wavelength shifting fibres which carry the signal to PMTs. The segmentation of the PEM and PHA towers is identical, and given in table 3.4. The PEM has 23 layers of lead, 
$4.5 \mathrm{~mm}$ thick, and 23 layers of $4 \mathrm{~mm}$ thick scintillator, corresponding to a thickness of 21 radiation lengths at normal incidence. The PHA has 23 layers of iron and scintillator, which are $2.5 \mathrm{~cm}$ and $6 \mathrm{~mm}$ thick respectively.

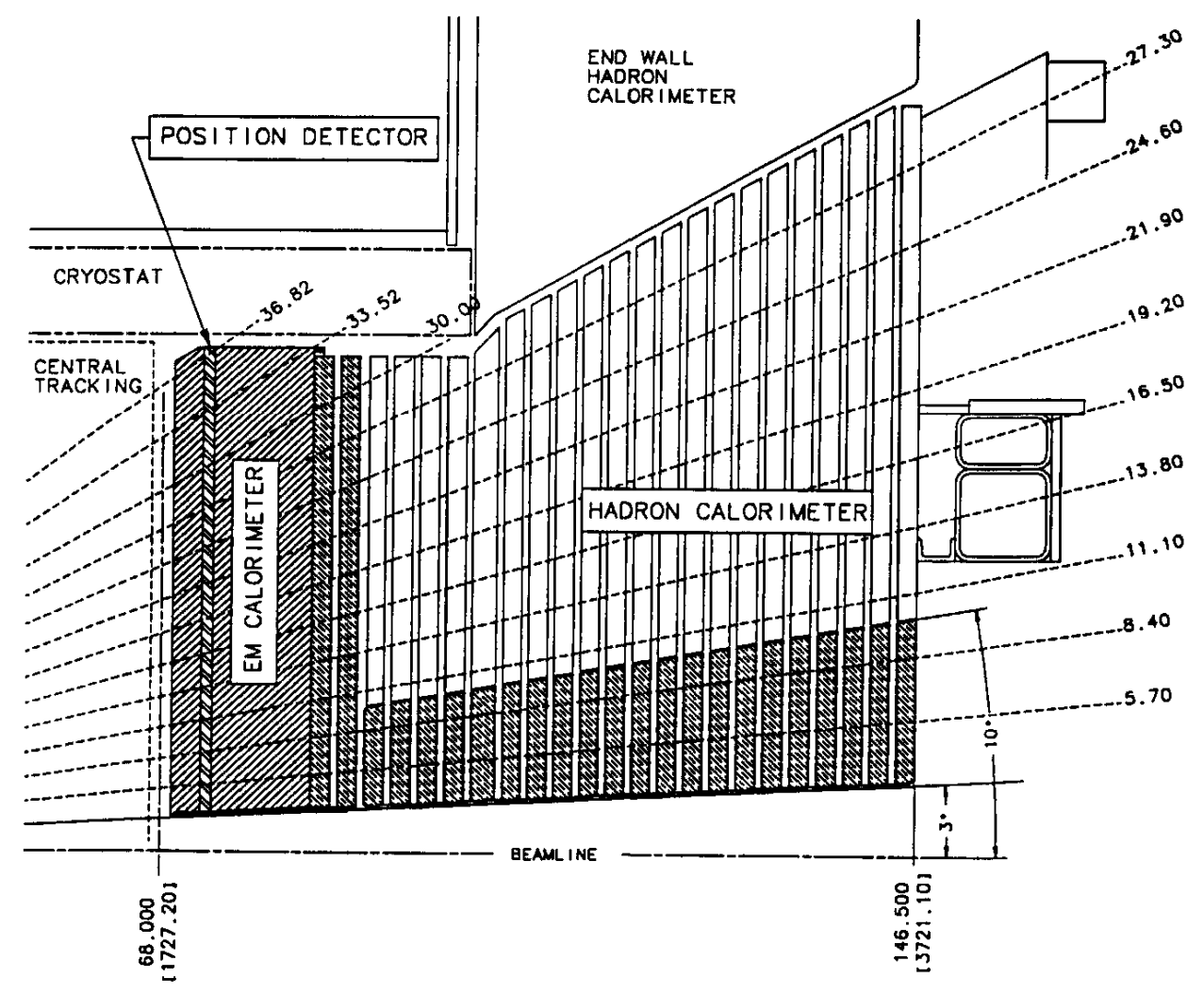

Figure 3.10: Cross-section view of top half of plug calorimeter

The Plug Electromagnetic Shower (PES) [30] detector is embedded inside the PEM at the shower maximum, at a depth of approximately 6 radiation lengths, out of a total of 23.2. The PES is divided into eight $45^{\circ}$ sectors, each covering the region from the beam pipe at $11 \mathrm{~cm}$ to an outer radius of $130 \mathrm{~cm}$. Each sector consists of two layers (called $\mathrm{U}$ and $\mathrm{V}$ ) of $5 \mathrm{~mm}$ pitch scintillator 
strips as shown in Figure 3.11. The strips in the two layers are aligned at $+22.5^{\circ}$ and $-22.5^{\circ}$ with respect to the radial direction from the beamline. Together the two layers provide two-dimensional position measurement. The PES has a position resolution of $(38 / E+0.4) \mathrm{mm}$ for the energy range 10 $-180 \mathrm{GeV}[44]$.

\begin{tabular}{|c|c|c|c|c|}
\hline \hline Tower & Tile ID & $\Delta \eta$ & $\Delta \theta$ & $\Delta \phi$ \\
\hline 10 & EM Only & $1.10-1.20$ & $33-37^{\circ}$ & $7.5^{\circ}$ \\
11 & 17,18 & $1.20-1.32$ & $30-33^{\circ}$ & $7.5^{\circ}$ \\
12 & 15,16 & $1.32-1.41$ & $27-30^{\circ}$ & $7.5^{\circ}$ \\
13 & 13,14 & $1.41-1.52$ & $25-27^{\circ}$ & $7.5^{\circ}$ \\
14 & 11,12 & $1.52-1.64$ & $22-25^{\circ}$ & $7.5^{\circ}$ \\
15 & 9,10 & $1.64-1.78$ & $19-22^{\circ}$ & $7.5^{\circ}$ \\
16 & 7,8 & $1.78-1.93$ & $16-19^{\circ}$ & $7.5^{\circ}$ \\
17 & 5,6 & $1.93-2.11$ & $14-16^{\circ}$ & $7.5^{\circ}$ \\
18 & 4 & $2.11-2.33$ & $11-14^{\circ}$ & $15^{\circ}$ \\
19 & 3 & $2.33-2.61$ & $8-11^{\circ}$ & $15^{\circ}$ \\
20 & 2 & $2.61-3.00$ & $6-8^{\circ}$ & $15^{\circ}$ \\
21 & 1 & $3.00-3.64$ & $3-6^{\circ}$ & $15^{\circ}$ \\
\hline
\end{tabular}

Table 3.4: The transverse tower segmentation of the upgraded plug calorimeter. [30]. 


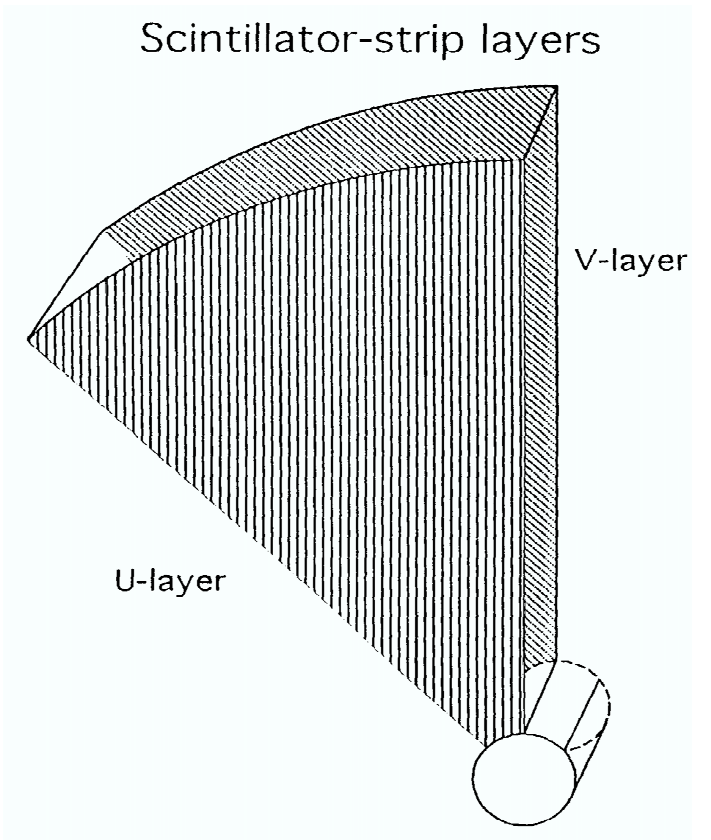

Figure 3.11: Schematic diagram of one of the eight PES sectors showing the direction of the strips in the $\mathrm{U}$ and $\mathrm{V}$ layers.

\subsubsection{Muon Systems}

The muon chambers are located outside the calorimetry. The central muon system is made up of three individual muon chambers, each of which consists of four layers of rectangular drift tubes with single wires. The three chambers are the central muon chamber (CMU)[30], the central muon chamber upgrade $(\mathrm{CMP})[46]$ and the central muon extension (CMX)[47]. These are described later. The CMU and the CMP cover $84 \%$ and $63 \%$ of solid angle for $|\eta|<0.6$ respectively, with an overlap region of $53 \%$ of solid angle. The CMX covers $71 \%$ of solid angle from $0.6<|\eta|<1.0$. The design parameters of each system are given in table 3.5. The coverage in $\phi$ and $\eta$ is shown in Figure 3.12. A fourth muon chamber, the IMU, provides coverage in the forward region but will not be discussed, as it is not used for this analysis. The nomenclature used for a track reconstructed in the muon chambers is a "muon stub". 


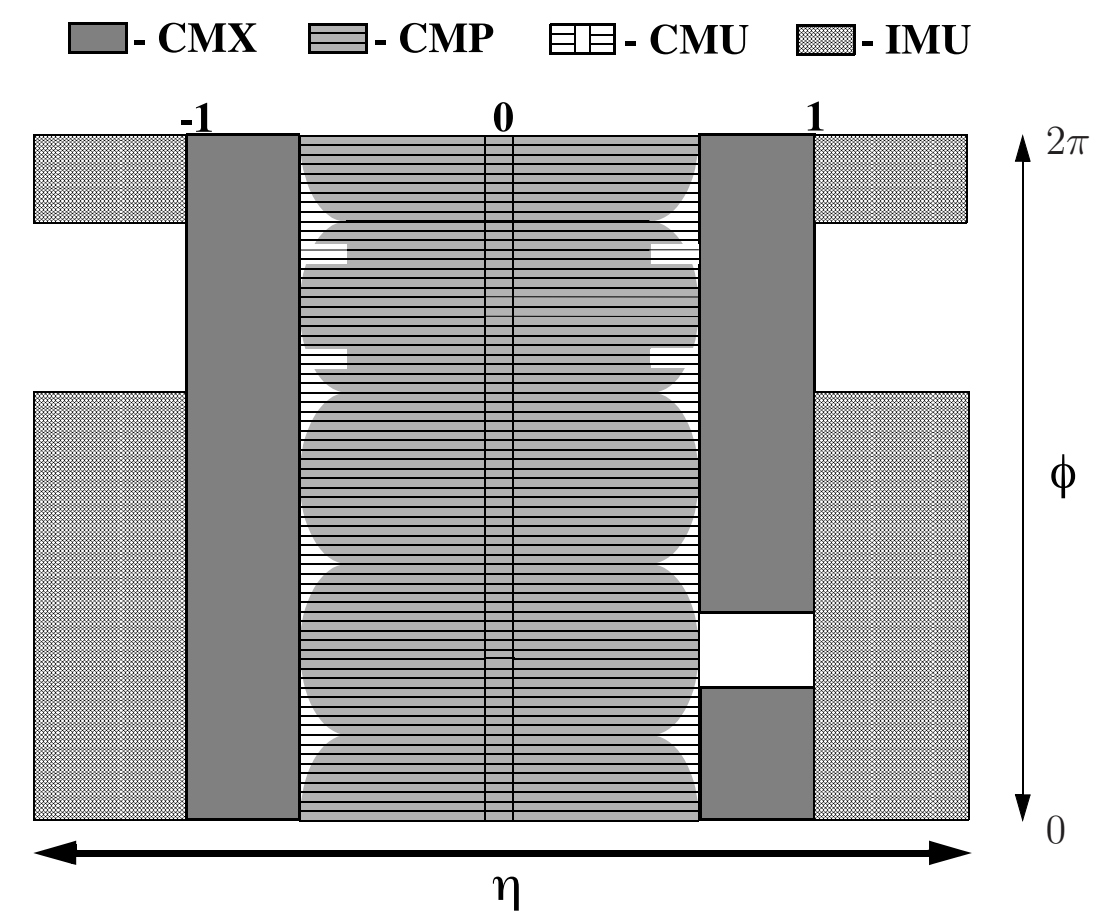

Figure 3.12: Location of the central muon detectors in azimuth $\phi$ and pseudorapidity $\eta$. On the east side, there is a gap in coverage in the CMX of $30^{\circ}$ in azimuth, due to the location of the cryogenic utilities servicing the solenoid. 


\section{Central muon chamber(CMU)}

The CMU is unchanged from Run I. It is located behind the towers of the CHA and divided into wedges covering $12.6^{\circ}$ in azimuth for $|\eta|<0.6$. Only muons with a $p_{T}>1.4 \mathrm{GeV} / c$ reach the CMU. Each wedge has three towers, each comprised of four layers of four drift tubes. The second and fourth layers are offset by $2 \mathrm{~mm}$ from the first and third as shown in Figure 3.13. A $50 \mu \mathrm{m}$ diameter stainless steel resistive sense wire is located in the centre of each cell. The wires in the cells in the first and third (second and fourth)

\begin{tabular}{|l|c|c|c|}
\hline \hline & CMU & CMP & CMX \\
\hline Pseudo-rapidity coverage & $|\eta| \leq 0.6$ & $|\eta| \leq 0.6$ & $0.6 \leq|\eta| \leq 1.0$ \\
Drift tube cross-section & $2.68 \times 6.35 \mathrm{~cm}$ & $2.5 \times 15 \mathrm{~cm}$ & $2.5 \times 15 \mathrm{~cm}$ \\
Drift tube length & $226 \mathrm{~cm}$ & $640 \mathrm{~cm}$ & $180 \mathrm{~cm}$ \\
Max drift time & $800 \mathrm{~ns}$ & $1.4 \mu \mathrm{s}$ & $1.4 \mu \mathrm{s}$ \\
Total drift tubes & 2304 & 1076 & 2208 \\
Scintillator counter thickness & & $2.5 \mathrm{~cm}$ & $1.5 \mathrm{~cm}$ \\
Scintillator counter width & & $30 \mathrm{~cm}$ & $30-40 \mathrm{~cm}$ \\
Scintillator counter length & & $320 \mathrm{~cm}$ & $180 \mathrm{~cm}$ \\
Total counters & & 269 & 324 \\
Pion interaction lenghts & 5.5 & 7.8 & 6.2 \\
Minimum detectable muon $P_{T}$ & $1.4 \mathrm{GeV} / \mathrm{c}$ & $2.2 \mathrm{GeV} / \mathrm{c}$ & $1.4 \mathrm{GeV} / \mathrm{c}$ \\
Multiple scattering resolution & $12 \mathrm{~cm} / p(\mathrm{GeV} / p)$ & $15 \mathrm{~cm} / p$ & $13 \mathrm{~cm} / p$ \\
\hline
\end{tabular}

Table 3.5: Design parameters of the CDF muon detectors. Pion interaction lengths and multiple scattering are computed at a reference angle of $\theta=90^{\circ}$ in CMU and CMP, and at an angle of $\theta=55^{\circ}$ in the CMX [30]. 
layers are connected in the readout. Each wire pair is instrumented with a time-to-digital converter (TDC) to measure the $\phi$-position of the muon and an analogue-to-digital converter (ADC) on each end to measure $z$ position via charge division. The position resolution of the detector is $250 \mu \mathrm{m}$ in the drift direction $(r-\phi)$ and $1.2 \mathrm{~mm}$ in the sense wire direction $(z)$.

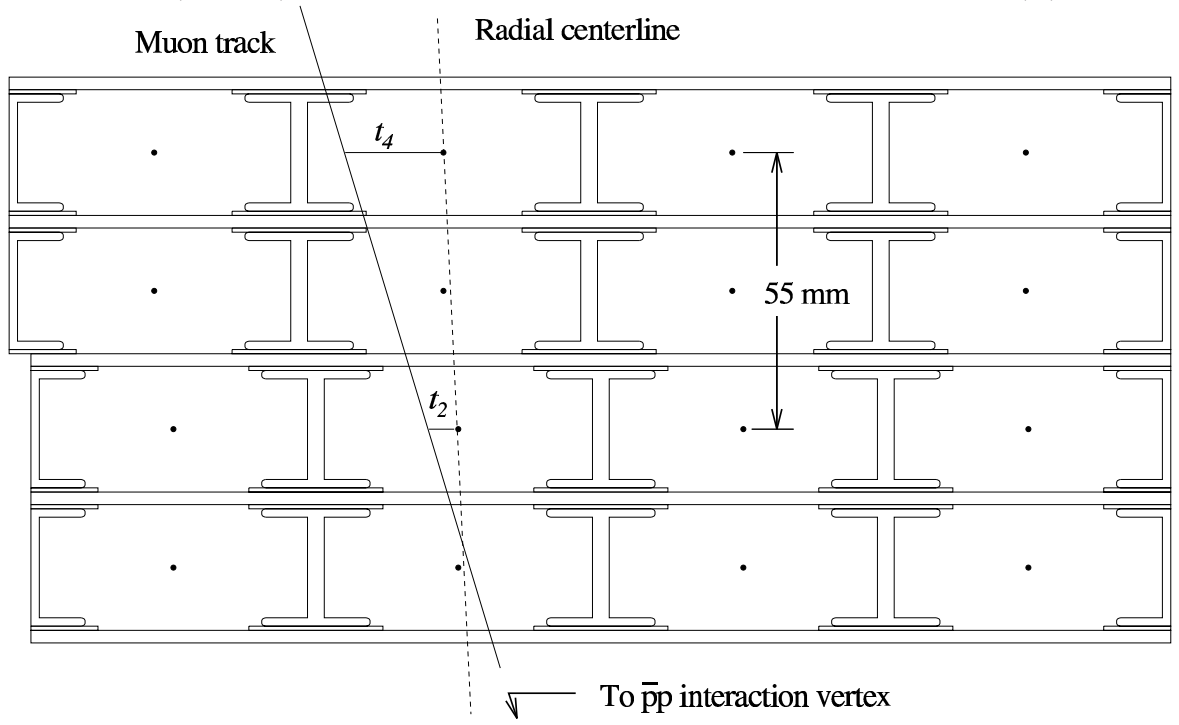

Figure 3.13: A CMU module in the $r-\phi$ plane with 4 layers of drift chambers. The drift times $t_{1}$ and $t_{2}$ are used to calculate muon momentum for triggering. Shown as a black circles, are the sense wires connected to the readout.

\section{Central muon upgrade(CMP)}

Approximately $0.5 \%$ of high energy hadrons produced, will pass through the CMU creating an irreducible fake muon background. In order to reduce this effect, an additional muon chamber (CMP) is installed behind $60 \mathrm{~cm}$ of steel. The CMP consists of a four-sided box placed on the outside of the CDF detector. Muons of a $p_{T}>2.5 \mathrm{GeV} / \mathrm{c}$ can reach the CMP. The rectangular 
form of the CMP detector means that its pseudo-rapidity varies in azimuth, as shown in Figure 3.12 .

\section{Central muon extension(CMX)}

The CMX extends the $\eta$ coverage to \pm 1.0 . It consists of two $120^{\circ}$ arches located at each end of the central detector, as shown in Figure 3.14. The uninstrumented regions have been filled by the insertion of a $30^{\circ}$ "keystone" at the top, and two $90^{\circ}$ "miniskirts" for the lower gaps. There is a gap in the coverage on the east side due to cryogenic utilities servicing the solenoid as shown in Figure 3.12, known as the chimney. The mini-skirt or keystone regions are defined as CMX wedges 5-6, and 15-20.

\subsubsection{Cherenkov Luminosity Counters}

The rate of inelastic scatter of $p \bar{p}$ interactions can be used to determine the luminosity $(\mathcal{L})$. A gas Cherenkov Luminosity Counter (CLC)[49] measures the number of interactions per beam crossing to determine the luminosity of the Tevatron. There are two CLCs positioned between the beam-pipe and the plug calorimeters, covering the region $3.7<|\eta|<4$.7. Each CLC consists of 48 thin, conical gas-filled Cherenkov counters. They are arranged in three concentric circles, each consisting of 16 counters.

The luminosity of a $p \bar{p}$ collider can be estimated using the equation:

$$
\mathcal{L}=\frac{f \times \mu}{\sigma}
$$

where $f$ is the frequency of bunch crossing, $\mu$ is the average number of interactions per beam crossing, given by the CLC hit rate (about 5-6), and $\sigma$ is the inelastic cross-section of $p \bar{p}$ scattering. The average of the inelastic 


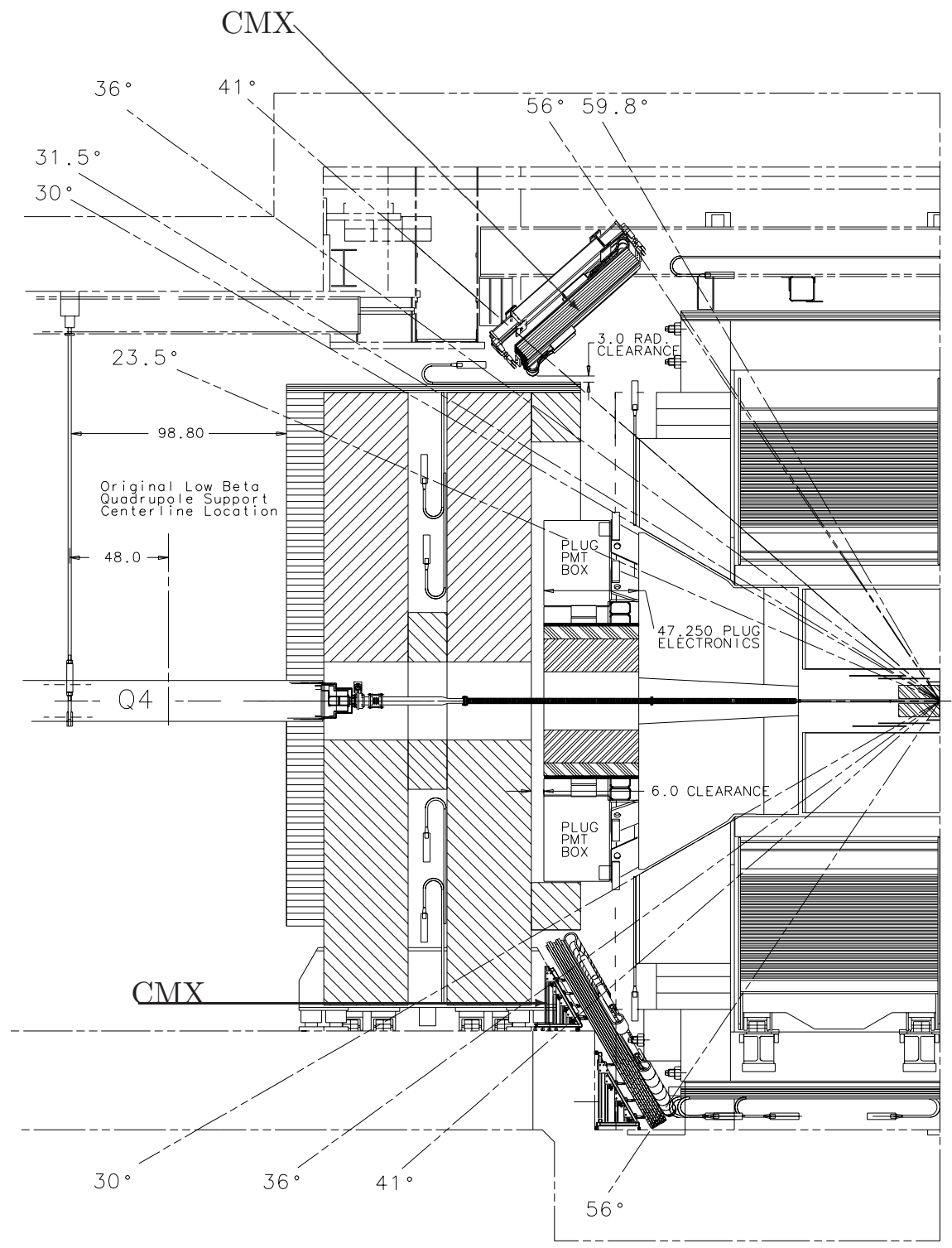

Figure 3.14: Side view of the CDF detector showing the placement of the CMX detectors. 
cross-sections as measured by CDF Run 1 and the E811[50] experiments is $60.7 \pm 2.3 \mathrm{mb}[48]$.

A total systematic uncertainty of $6 \%$ is quoted for all luminosity measurements. This includes a $4.4 \%$ contribution from the acceptance and operation of the luminosity monitor and $4.0 \%$ from the theoretical uncertainty on the calculation of the total $p \bar{p}$ cross-section[48].

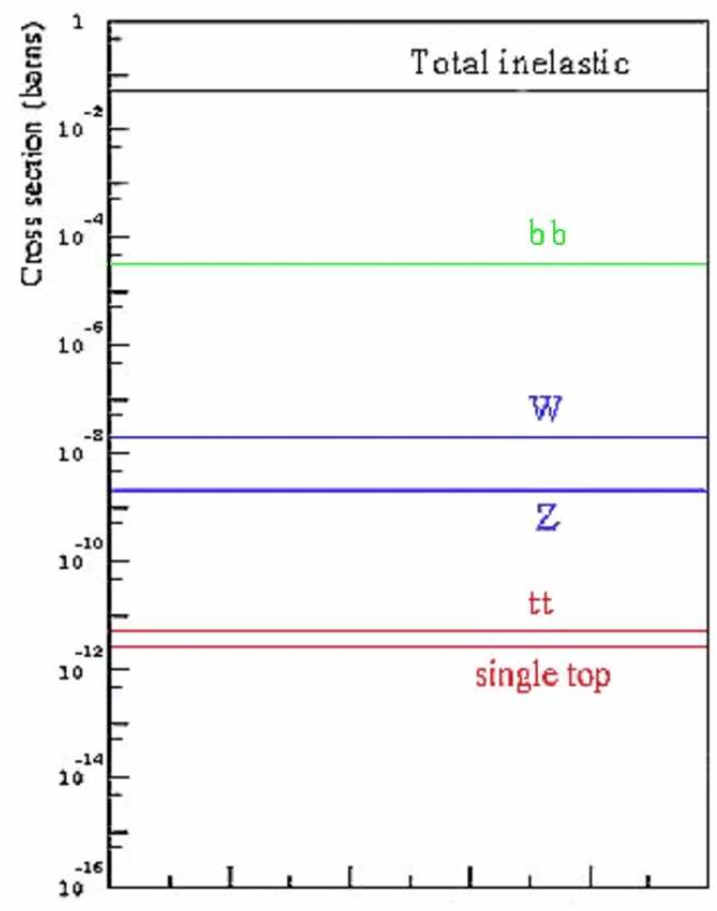

Figure 3.15: The relative production cross-sections for different processes at CDF. 


\subsection{Trigger and Data Acquisition}

Many interesting physics processes have cross sections which are many orders of magnitude smaller than the total inelastic cross section, as shown in figure 3.15. These events must be triggered because the collision rate at the Tevatron is much higher than the rate at which data can be recorded. CDF has a three tier trigger system. Each level reduces the rate of data, to a level which is sufficient to allow processing at the subsequent level with minimal dead-time.

The Level 1 hardware trigger looks for physics objects such as clusters in the EM-cal or track segments in muon chambers. This trigger makes a decision within $4 \mu \mathrm{s}$. This trigger reduces the event rate from $7.6 \mathrm{MHz}$ to 50 KHz. Accepted events are then passed to the Level 2 hardware. The Level 2 system uses jet clustering as well as improved momentum resolution for tracks, finer angular matching between muon stubs and central tracks and data from the central shower-max detector (CES) for improved identification of leptons and photons. The Level 3 trigger fully reconstructs the events in a processor farm using full detector information. Data which passes one of the specified trigger paths are reconstructed using the latest calibrations on a computer cluster and written to tape. The Level 3 trigger takes 300 events per second from the Level 2 Trigger, and writes to tape at a rate of $\leq 50 \mathrm{~Hz}$. 


\section{Chapter 4}

\section{$Z^{0}$ boson Selection}

The philosophy of this analysis is to select a $Z^{0}$ decaying to $e^{+} e^{-}$or $\mu^{+} \mu^{-}$, and then to look for a photon in addition. In this chapter, the event selection for the inclusive $Z^{0}$ sample is detailed. A requirement on the primary vertex of the event is given in section 4.1. The selection of $Z^{0} \rightarrow e^{+} e^{-}$and $Z^{0} \rightarrow$ $\mu^{+} \mu^{-}$candidates is given in sections 4.2 and 4.3 , respectively.

\subsection{Event Vertex}

The primary vertex is given by, $z_{0}$, the distance in $z$ from the nominal interaction point $(z=0)$ to the $z$ value given by where the track of the tight lepton, crosses the beamline. To ensure the tracks are contained within the fiducial region of the tracking systems, the primary vertex is required to be within $60 \mathrm{~cm}$ of the nominal interaction region. The efficiency of this cut, $\epsilon_{z 0}$, has been measured elsewhere to be $\epsilon_{z 0}=95 \pm 0.4 \%$ [59] in data.

In the $\mathrm{MC}$ samples, only events with a true vertex $<60 \mathrm{~cm}$ are used. I.e. this cut is $100 \%$ efficient in MC. 


\section{2 $\quad Z^{0} \rightarrow e^{+} e^{-}$Selection}

In this analysis, an "electron" will be used to refer to both $e^{+}$and $e^{-}$. Three categories of electrons will be used, selected using different selection criteria. These will be refered to as "tight", "loose" and "plug" electrons. The triggering of the high $P_{T}$ electron dataset is described in section 4.2.1, with a description of the data quality requirements given in section 4.2.2. The variables used for electron identification are detailed in section 4.2.3, a detailed summary of the selection used for the different categories of electrons are given in tables 4.3 and 4.4. The cuts are shown graphically in section 4.2.4, with the distributions for the different identification variables, and their efficiencies in section 4.2.5. The total $Z^{0}$ selection efficiency is described in section 4.2.7.

\subsubsection{Electron Trigger Sample}

The $Z^{0} \rightarrow e^{+} e^{-}$measurements require a central electron to pass the high transverse momentum $\left(P_{T}\right)$ electron ELECTRON_CENTRAL_18 trigger[51]. As discussed in section 3.4, a three tier trigger system is used.

- At Level 1, an online tracking algorithm known as the "eXtremely Fast Tracker" or XFT[52], is used to construct tracks with hits in a number of layers $\left(N_{\text {layers }}^{X F T}\right)$ in the COT and measure the transverse momentum $\left(P_{T}^{X F T}\right)$. The transverse energy $\left(E_{T}\right)$ and the ratio of the energy deposits in the hadronic and electromagnetic calorimeters $\left(E_{\text {had }} / E_{\text {em }}\right)$ is measured on a per tower basis.

- At Level 2, a clustering algorithm is used to construct energy clusters 
associated with electrons [55]

- At Level 3, electromagnetic energy clusters[60] (referred to as an EMobjects) are made using a seed tower and one or two adjacent towers.

The requirements of the level 1,2 and 3 trigger requirement comprising the ELECTRON_CENTRAL_18 trigger are summarised in Table 4.1. This trigger has an efficiency of $96.3 \pm 1 \%$ [57] for central electrons with $E_{T}>20 \mathrm{GeV}$.

\begin{tabular}{|c|c|}
\hline Trigger Level & Requirement \\
\hline 1 & $\begin{array}{c}\text { A single tower with } E_{T} \geq 8 \mathrm{GeV} \\
\text { A single tower with } E_{\text {had }} / E_{e m} \leq 0.125 \\
\text { An XFT track pointing to tower with } P_{T}^{X F T} \geq 8.34 \mathrm{GeV} / c \\
\text { An XFT track pointing to tower with } N_{\text {layers }}^{X F T} \geq 3\end{array}$ \\
\hline 2 & $\begin{array}{c}\text { A central energy cluster with } E_{T} \geq 16 \mathrm{GeV} \\
\text { A central energy cluster with } E_{h a d} / E_{e m} \leq 0.125 \\
\text { An XFT track pointing to seed tower with } P_{T}^{X F T} \geq 8.34 \mathrm{GeV} / c\end{array}$ \\
\hline 3 & $\begin{array}{c}\text { A central EMobject with } E_{T} \geq 18 \mathrm{GeV} \\
\text { A central EMobject with } E_{\text {had }} / E_{e m} \leq 0.125 \\
\qquad N_{E M o b j e c t} \geq 1 \\
\text { Central Track } P_{T} \geq 9 \mathrm{GeV} / c\end{array}$ \\
\hline
\end{tabular}

Table 4.1: ELECTRON_CENTRAL_18 Trigger requirements.

\subsubsection{Good run list}

Requirements are placed on the subdetector and trigger operational status to ensure optimal data quality. "Good" status are required for the COT, calorimeter and showermax systems[53]. For the $Z_{C P}^{0}$ analysis, the silicon 
system is also required to have good status, due to the use of PHOENIX tracking.

The triggers, datasets and luminosities for the individual analysis channels are listed in Table 4.2, after the good run requirements have been applied.

\begin{tabular}{|l|r|r|}
\hline Sample & L3 Trigger & $\mathcal{L}\left(\mathrm{pb}^{-1}\right)$ \\
\hline$Z^{0} \gamma \rightarrow e e$ CC & ELECTRON_CENTRAL_18 & $202 \pm 12$ \\
$Z^{0} \gamma \rightarrow e e$ CP & ELECTRON_CENTRAL_18 & $168 \pm 10$ \\
\hline
\end{tabular}

Table 4.2: L3 Trigger Path, Dataset name and integrated luminosity $(\mathcal{L})$ for the electron channels. There is a $6 \%$ systematic error quoted on the luminosity, see section 3.3.6.

\subsubsection{Electron Identification Variables}

A large number of identification variables is used to select electron candidates:

- Track transverse momentum

$P_{T}$ is the transverse momentum of the track associated with the electron. For the central(plug) electron, a COT(PHOENIX) tracking algorithm is used as described in section 3.3.2. Momentum resolution is improved by constraining the track to originate from the beam line. A $P_{T}$ of at least $10 \mathrm{GeV} / c$ is required.

- To ensure a well measured COT track for a central electron, seven hits, in at least three of the axial $(A x)$ and stereo $(S t)$ superlayers 
$(S L)$ of the COT, are required. The number of axial(stereo) superlayers which meet this requirement is referred to as $N_{A x}\left(N_{S t}\right)$.

- At least three hits are required in the silicon detectors for a PHOENIX track.

- Fiducial region requirement

Central (plug) electrons must lie within $|\eta|<1.1(1.2 \leq|\eta| \leq 2.6)$. The electron position inside a CEM tower is measured using the $\phi(z)$ position of the shower as measured by the CES detector, $X_{C E S}\left(Z_{C E S}\right)$. The following three requirements are made to ensure the electron is reconstructed in an active, well instrumented region of the detector.

1. $\left|X_{C E S}\right|<21 \mathrm{~cm}$ where $X_{C E S}$ is measured from the centre of the tower.

2. $9<\left|Z_{C E S}\right|<230 \mathrm{~cm}$.

3. The region $0.77<\eta<1.0,75^{\circ}<\phi<90^{\circ}$ and $\left|Z_{C E S}\right|>193 \mathrm{~cm}$ is excluded ${ }^{1}$.

- Electromagnetic energy clusters, $E$ and $E_{T}$

An electromagnetic energy cluster[60], or EMobject, is made of a seed tower and one or two adjacent towers.

The total amount of energy for a cluster in the electromagnetic calorimeter $(E)$ is calculated by adding the electromagnetic $\left(E_{e m}\right)$ and hadronic ( $\left.E_{\text {had }}\right)$ energy components. It is used to define $E_{T}$, the component of energy transverse to the beamline, given by $E \times \sin \theta$. The minimum $E_{T}$ requirement for the seed (adjacent) tower is $3 \mathrm{GeV}(100 \mathrm{MeV})$. A

\footnotetext{
${ }^{1}$ Uninstrumented region to allow cryogenic servicing to the solenoid.
} 
successful central (plug) electron candidate must have $E_{T}>25(20)$ $\mathrm{GeV}$.

- Ratio of hadronic/electromagnetic energy

An energetic electron produced at the centre of the detector will travel through the tracking systems before depositing most of its energy in the electromagnetic calorimeter with only a small amount of leakage into the hadronic calorimeter. $E_{\text {had }} / E_{\text {em }}$ must be less than $0.055+$ $0.00045 \times E$.

- Calorimeter Isolation

Background, such as jets, can be suppressed by placing isolation requirements on the electron candidate. A cone of $\Delta R=\sqrt{\Delta \phi^{2}+\Delta \eta^{2}}<$ 0.4 , is built around the electron's energy cluster seed position, as determined by the CES detector. A limit can then be placed on the $E_{T}$ sum in the cone (Iso4), excluding the energy cluster of the electron itself. The requirement is made that $I s o 4 / E_{T}<0.1$.

- Consistency with electromagnetic shower deposition

$$
-L_{s h r}
$$

The "lateral shower sharing variable", $L_{s h r}$, is a measure of consistency between the observed distribution of the energy deposits to that expected from a genuine electromagnetic shower. $L_{s h r}$ is defined as[56]:

$$
L_{s h r}=0.14 \frac{\sum_{i}\left(M_{i}-P_{i}\right)}{\sqrt{\left(0.14 \sqrt{E_{e m}}\right)^{2}+\sum_{i}\left(\Delta P_{i}\right)^{2}}}
$$


where the sums are over the towers $(i)$ in the cluster adjacent to the seed tower, $M_{i}$ is the measured energy deposit, $P_{i}$ is the predicted energy deposit calculated using a parametrisation from test-beam data, $E_{\text {em }}$ is the total electromagnetic energy in the cluster and $\Delta P_{i}$ is an estimate of the uncertainty on $P_{i}$. The error on the energy measurement is $0.14 \sqrt{E_{e m}}$. Electron candidates have $L_{s h r}<0.2$.

$-\operatorname{PEM} 3 \times 3 \chi^{2}$

The PEM $3 \times 3 \chi^{2}$ variable compares the energy distribution for nine towers, arranged in a $3 \times 3$ square around the seed tower, to energy distributions from test beam electrons, for the plug calorimeter. The PEM $3 \times 3 \chi^{2}$ value is required to be less than 10.

- CES and PES Cluster profile

$-\chi_{\text {strip }}^{2}$

The $\chi^{2}$ comparison of the CES shower profile in the $r-z$ view with the same profile extracted from test beam electrons is used to distinguish between electromagnetic showers from prompt photon and electrons and those from jets (where typically $\pi^{0} \rightarrow \gamma \gamma$ ). Details of this parameter can be found in section 7.4.1. The corresponding $r-\phi$ variable, $\chi_{w i r e}^{2}$, is not used for electron identification as it is sensitive to bremstrahlung radiation. The $\chi^{2}$ value is required to be less than 10 for clusters in the CES.

- PES 5by9, U and V

The "5by9" ratio can be used to give a brief description of the 
shape of the cluster. A five strip window is placed around a PES cluster in the $\mathrm{U}$ or $\mathrm{V}$ wires (see figure 3.11). A corresponding nine strip window consists of these five strips plus two more strips on each side. The ratio of the energy recorded in the 5 strips, to that recorded in the 9 strips can then be used to give a description of the cluster profile. The PES 5by9 ratio is required to be less than 0.65 for both the $\mathrm{u}$ and $\mathrm{v}$ strips (if $E_{T}<100 \mathrm{GeV}$ ).

- $Q \cdot \Delta x$ and $\Delta z$

The distance between the extrapolated track and the best matching CES cluster in the $r-\phi$ and $r-z$ planes are $\Delta x$ and $\Delta z$ respectively. The cut on $\Delta x$ is multiplied by the charge. $Q$, of the track. An asymmetric cut is applied to account for bremsstrahlung radiation which distorts the CES cluster in a charge-dependent direction. An electron candidate must have $-3.0<q \cdot \Delta X<1.5 \mathrm{~cm}$ and $|\Delta Z|<3 \mathrm{~cm}$.

- $E / P$

An electron travelling through the tracking volume of the detector may radiate a photon. This photon will generally enter in the same tower as the electron, not affecting the measured $E$ of the electron. However, this radiation will reduce the measured $P_{T}$ of the electron causing a high tail in the distribution of $E / P$. For the tight central electron the ratio of $E / P$ where $P$ is the momentum of the electron as measured by the tracking systems must be less than 2 .

The tight, loose and plug electron cuts are summarised in tables 4.3 and 4.4 . 


\begin{tabular}{|c|c|}
\hline \hline Tight Central Electron & Loose Central Electron \\
\hline$E_{T}>25 \mathrm{GeV}$ & $E_{T}>25 \mathrm{GeV}$ \\
$|\eta|<1.1$ & $|\eta|<1.1$ \\
$P_{T}>10 \mathrm{GeV}$ & $P_{T}>10 \mathrm{GeV}$ \\
$N_{A x} \mathrm{SL} \geq 3, N_{S t} \mathrm{SL} \geq 3$, with $\geq 7$ hits & $N_{A x} \mathrm{SL} \geq 3, N_{S t} \mathrm{SL} \geq 3$, with $\geq 7 \mathrm{hits}$ \\
$E_{h a d} / E_{\text {em }}<0.055+0.00045 \cdot E$ & $E_{h a d} / E_{\text {em }}<0.055+0.00045 \cdot E$ \\
$E / P<2|| P_{T}>50 \mathrm{GeV}$ & \\
$I s o 4 / E_{T}<0.1$ & $I s o 4 / E_{T}<0.1$ \\
$\chi_{\mathrm{Strip}}^{2}<10$ & \\
$-3.0<q \cdot \Delta X<1.5 \mathrm{~cm}$ & \\
$|\Delta Z|<3 \mathrm{~cm}$ & \\
$L_{\text {shr }}<0.2$ & \\
$\left|X_{C E S}\right|<21.0 \mathrm{~cm}$ & \\
$9.0<\left|Z_{C E S}\right|<230.0 \mathrm{~cm}$ & \\
\hline \hline
\end{tabular}

Table 4.3: Table of cuts to select tight and loose central electrons.

\begin{tabular}{|l|l|}
\hline Variable & Requirement \\
\hline$E_{T}$ & $>20 \mathrm{GeV}$ \\
Fiducial & $1.2<|\eta|<2.8$ \\
$\mathrm{Had} / \mathrm{Em}$ & $<0.05$ for $E \leq 100 \mathrm{GeV}$ \\
& $<0.05+0.026 \cdot \ln (E / 100)$ for $E>100 \mathrm{GeV}$ \\
$I$ so4 $/ E_{T}$ & $<0.1$ \\
PES $5 \times 9$ u and v & $>0.65$ for $E_{T}<100 \mathrm{GeV}$ \\
PEM3x3 $\chi^{2}$ & $<10$ \\
PEM Fit Towers & $>0$ \\
Phoenix 2D Track & yes \\
NSvx Hits & $>=3$ \\
\hline
\end{tabular}

Table 4.4: Criteria used in the selection of the plug electron. 


\subsubsection{N-1 Plots}

The effectiveness of the electron selection cuts, described in section ??, is best demonstrated in "N-1" plots. After selecting events with one tight central electron, all but one of the $N$ tight cuts are applied to the second electron, ensuring a pure sample of electrons ${ }^{2}$. The distribution of the $N^{t h}$ variable is then plotted for the second electron. Comparisons made between the high $P_{T}$ electron data and the $Z^{0} \rightarrow e^{+} e^{-}$Monte Carlo simulation (described in section 2.2.5) are shown in Figures 4.1 and 4.2 for the central electron candidates.

In Figure 4.1, both the $E_{T}$ and $P_{T}$ distributions peak at about $40 \mathrm{GeV}$, just under half the mass of the $Z^{0}$ boson. Correspondingly, the $E / P$ distribution shows a very narrow peak at 1, with a tail to higher energies, modelled correctly by the simulation, due to bremsstrahlung radiation. Both the $E_{\text {had }} / E_{\text {em }}$ and $I s o 4 / E_{T}$ distributions are peaked at zero, demonstrating the electrons are very isolated and do deposit almost all their energy in the EM calorimeter.

In Figure 4.2, the asymmetric form of the $\Delta x \times Q$ variable can be seen compared to the gaussian form of the $\Delta z$ distribution. The $\chi^{2}$ distribution peaks at almost 0 for both data and MC. The MC simulation agrees well with the data for all distributions with the exception of the $L_{s h r}$ variable. The disagreement in the central bin of the $L_{s h r}$ distribution is due to a problem with the simulation which has been fixed in later versions of the simulation. This discrepancy causes no problem for this measurement since the cut is in the tail end of the distribution.

\footnotetext{
${ }^{2}$ Throughout this chapter "electron" refers to both electrons and positrons
} 
Figure 4.3 show the N-1 distributions for the plug electron candidates. Here one tight central electron is selected, and all but one of the $N$ plug electron cuts is applied to the second electron. The plug $E_{T}$ distribution shows that the cut of $20 \mathrm{GeV}$, removes background at low $E_{T}$ which are not consistent with electrons. Again both calorimeter isolation and $E_{\text {had }} / E_{\text {em }}$ distributions are peaked close to zero. The calorimeter isolation distribution peaks slightly lower in $\mathrm{MC}$ than data. However the cut value is located at 0.1 , away from the peak values at 0.02 and $0.03 \mathrm{GeV}$ for the $\mathrm{MC}$ and data respectively. The $E_{\text {had }} / E_{\text {em }}$ distribution shows good agreement between data and MC. The Pes 5by9 variable is poorly modelled by simulation. However, the cut is placed at a value of 0.65 , far away from the peak of 0.78 and 0.9 for data and MC respectively. The MC simulation predicts smaller PEM 3by3 $\chi^{2}$ values than is found in data. 

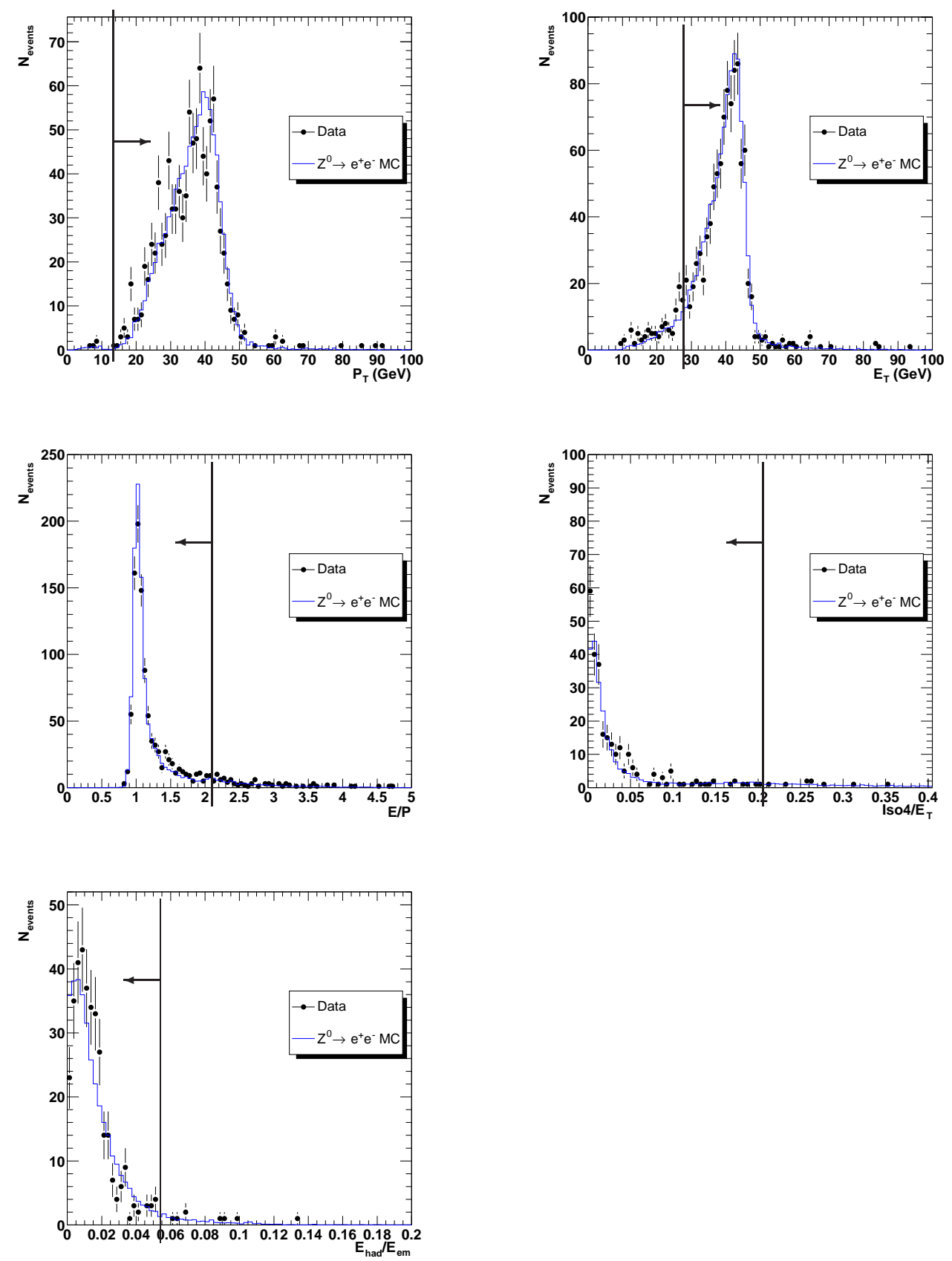

Figure 4.1: N-1 distributions for central electron variables used for the selection of the events, plotted for data (dots) and MC (solid histogram). The arrow shows graphically the selection cut. The MC simulation is normalised to the luminosity of the data. 

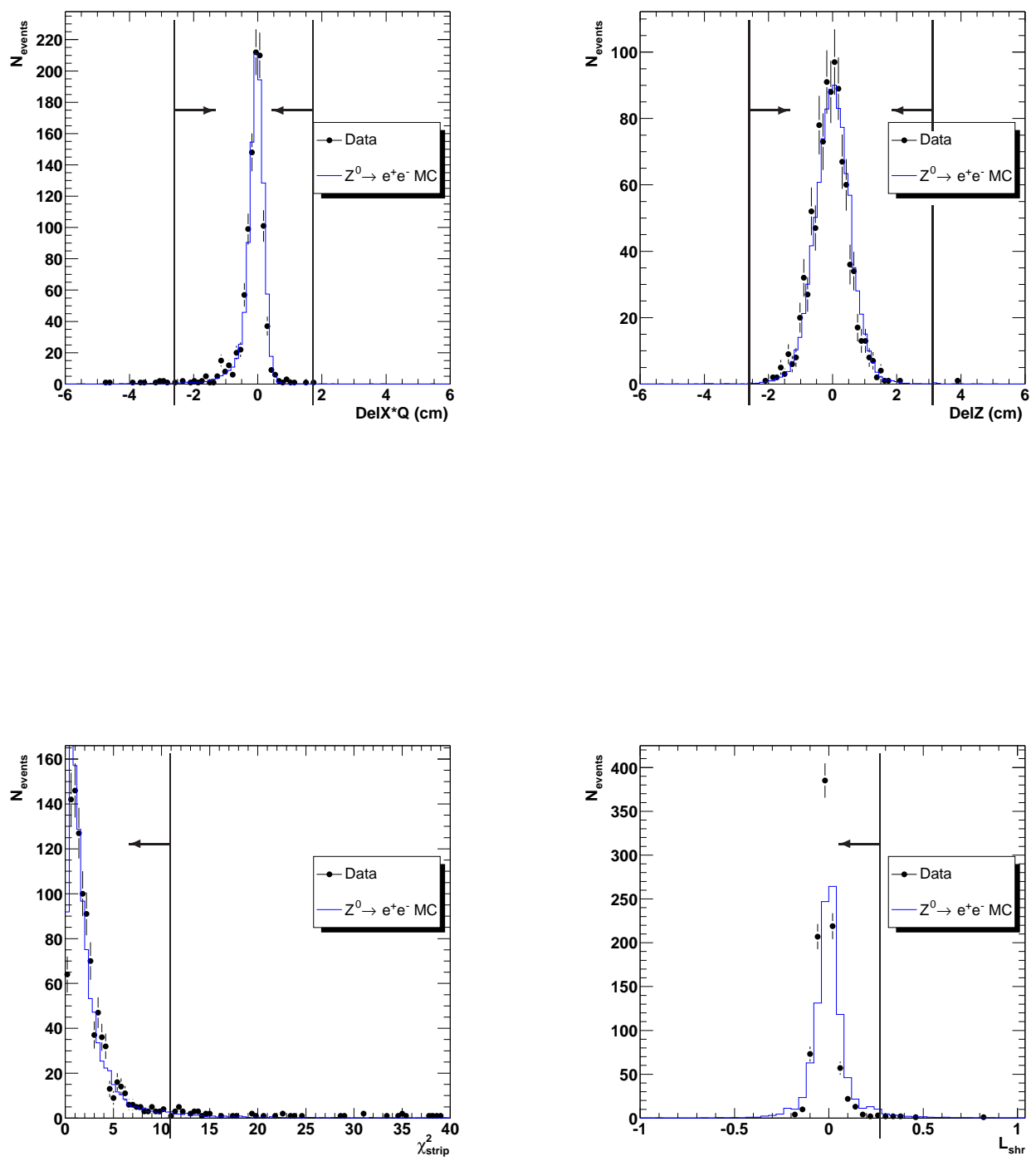

Figure 4.2: N-1 distributions for central electron variables used for the selection of the events, plotted for data (dots) and MC (solid histogram). The arrow shows graphically the selection cut. The MC simulation is normalised to the luminosity of the data. 


\subsubsection{Electron Identification Efficiencies}

The efficiency of photon and lepton selection is determined from Monte Carlo simulation. However, the simulation is imperfect. The following correction factors, $\frac{\epsilon_{D A T A}}{\epsilon_{M C}}$ are used to scale the MC to better represent the efficiency measured in data. A break down of the individual cut efficiencies is shown in Appendix A.1.

- The efficiency of the $z_{0}$ requirement $\left(\epsilon_{v t x}\right)$ is $95 \% \pm 0.4 \%\left(\epsilon_{z 0}\right)$, measured in data[59]. Only events with $\left|z_{0}\right|<60 \mathrm{~cm}$ are considered in Monte Carlo.

- The trigger efficiency $\left(\epsilon_{t r g}^{e}\right)$ is $96.3 \%$. (see section 4.2 .1 ).

- The $\frac{\epsilon_{D A T A}}{\epsilon_{M C}}$ ratio of the central electron selection efficiencies has been measured to be $96.4 \pm 1 \%$ for tight electron candidates $\left(\epsilon_{\text {tight }}\right)$, and $97.8 \pm$ $1 \%$ for loose electron candidates $\left(\epsilon_{\text {loose }}\right)[61]$.

- the $\frac{\epsilon_{D A T A}}{\epsilon_{M C}}$ ratio of the plug electron selection efficiencies $\left(\epsilon_{\text {plug }}\right)$ has been measured to be $94.2 \pm 1 \%$ [64]. The ratio of the efficiencies of the Phoenix track requirement is $98.6 \pm 0.6 \%$ [65]. Therefore the correction factor for plug electron ID is $0.929 \pm 0.013$.

- There is excellent agreement between the data and MC COT tracking efficiencies $\left(\epsilon_{t r k}\right)$. The correction factor is $1.000 \pm 0.004[62]$.

\subsection{6 $\quad \mathrm{Z}^{0} \rightarrow \mathrm{e}^{+} \mathrm{e}^{-}$Selection Efficiencies}

$Z^{0} \rightarrow e^{+} e^{-}$events are selected by requiring one "tight" electron, which passes the tight central electron cuts detailed in Table 4.3, and one "loose" electron. 

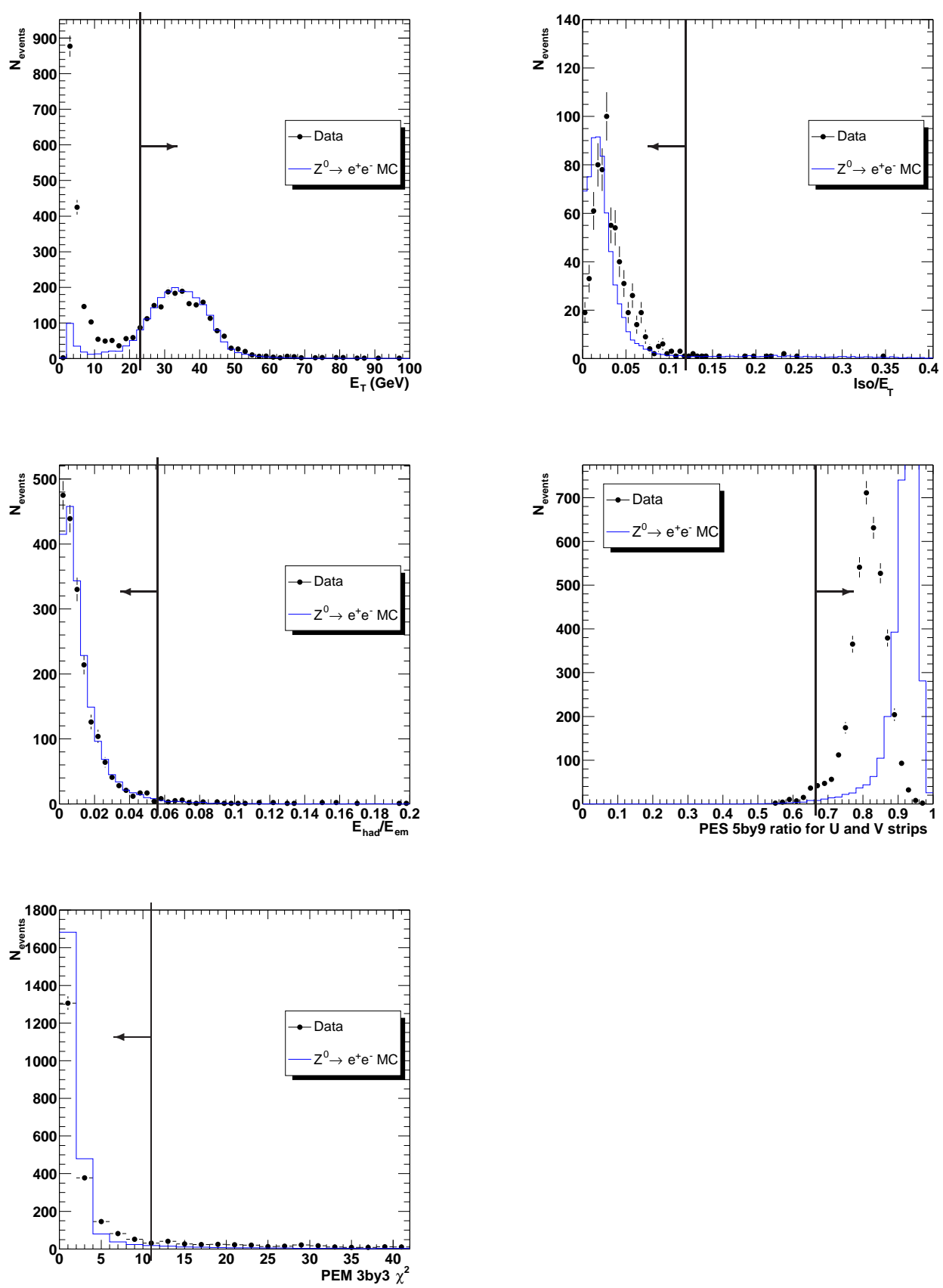

Figure 4.3: N-1 distributions for plug electron variables used for the selection of the events, plotted for data (dots) and MC (solid histogram). The arrow shows graphically the selection cut. The MC simulation is normalised to the luminosity of the data. 
The loose electron can either be a central electron passing the loose electron criteria of Table 4.3, or a plug electron passing the cuts in Table 4.2.3. Events with a loose central (plug) electron are referred to as $Z_{C C}^{0}\left(Z_{C P}^{0}\right)$ events.

For $Z_{C C}^{0}$ candidate events, either electron can be the trigger electron. The trigger efficiency for at least one of the $Z_{C C}^{0}$ electrons to trigger $\left(\epsilon_{t r g}^{Z}\right)$ is given by:

$$
\begin{aligned}
\epsilon_{t r g}^{Z} & =\epsilon_{t r g}^{e}+\left(1-\epsilon_{t r g}^{e}\right) \epsilon_{t r g}^{e} \\
& =\epsilon_{t r g}^{e}\left(2-\epsilon_{t r g}^{e}\right)
\end{aligned}
$$

In order for a $Z_{C C}^{0}$ to be reconstructed, at least one electron must be reconstructed as a tight electron and the other as a loose central electron. The total efficiency for a $Z_{C C}^{0}$ to be reconstructed is therefore:

$$
\begin{aligned}
\epsilon_{i d}^{Z} & =\epsilon_{\text {tight }}^{e} \cdot \epsilon_{\text {loose }}^{e}+\epsilon_{\text {loose }}^{e} \cdot \epsilon_{\text {tight }}^{e}-\left(\epsilon_{\text {tight }}^{e}\right)^{2} \\
& =\epsilon_{\text {tight }}^{e}\left(2 \epsilon_{\text {loose }}^{e}-\epsilon_{\text {tight }}^{e}\right) .
\end{aligned}
$$

Table 4.5 shows the calculation of the factor $\epsilon_{D a t a} / \epsilon_{M C}$ for the $Z_{C C}^{0}$ channel. A $Z_{C P}^{0}$ candidate consists of a central tight electron and a plug electron. These electrons are in mutually exclusive $\eta$ ranges and only the central electron can be the trigger electron. The total correction factor is therefore just the product of the individual terms, as summarised in Table 4.5.

\subsection{7 $\quad Z^{0}$ selection}

Both $Z_{C C}^{0}$ and $Z_{C P}^{0}$ selections require that the invariant mass of the electron pair must be in the range $40<m(l, l)<130 \mathrm{GeV}$. This mass range ensures that the the lepton pair are consistent with the decay of a $Z^{0}$ boson. In addition, the lower cut reduces the Drell-Yan contribution. 


\begin{tabular}{|l|c|c|c|c|}
\hline Efficiency & $\epsilon_{\text {Data }}$ & $\epsilon_{M C}$ & $\left(\epsilon_{\text {Data }} / \epsilon_{M C}\right)^{l}$ & $\left(\epsilon_{\text {Data }} / \epsilon_{M C}\right)^{Z}$ \\
& $(\%)$ & $(\%)$ & $(\%)$ & $(\%)$ \\
\hline$\epsilon_{\text {trg }}$ & $96.3 \pm 1.0$ & - & $96.3 \pm 1.0$ & $\epsilon_{\text {trg }}^{Z}=99.9 \pm 0.1$ \\
$\epsilon_{v t x}$ & $95.0 \pm 0.4$ & - & $95.0 \pm 1.0$ & $95.0 \pm 0.4$ \\
$\epsilon_{\text {tight }}$ & $82.5 \pm 0.5$ & $85.6 \pm 0.2$ & $96.4 \pm 1$ & \} \\
$\epsilon_{\text {loose }}$ & $94.1 \pm 0.3$ & $96.2 \pm 0.08$ & $97.8 \pm 1$ & $\epsilon_{\text {id }}^{Z}=95.6 \pm 0.6$ \\
\hline \multicolumn{2}{|l}{ Total Correction Factor } & $\frac{\epsilon_{\text {Data }}}{\epsilon_{M C}}=90.7 \pm 1.2 \%$ \\
\hline
\end{tabular}

Table 4.5: A summary of the efficiencies and correction factors used for the $Z_{C C}^{0}$ cross-section calculation. Details about $\epsilon_{\text {tight }}$ and $\epsilon_{\text {loose }}$ are found in [61].

The total correction factor is the product of the terms in the right column.

\begin{tabular}{|l|c|c|c|c|}
\hline Efficiency & $\begin{array}{c}\epsilon_{\text {Data }} \\
(\%)\end{array}$ & $\begin{array}{c}\epsilon_{M C} \\
(\%)\end{array}$ & $\begin{array}{c}\left(\epsilon_{\text {Data }} / \epsilon_{M C}\right)^{l} \\
(\%)\end{array}$ & $\begin{array}{c}\left(\epsilon_{\text {Data }} / \epsilon_{M C}\right)^{Z} \\
(\%)\end{array}$ \\
\hline$\epsilon_{\text {trg }}$ & $96.3 \pm 1.0 \%$ & - & $96.3 \pm 1.0 \%$ & $96.3 \pm 1.0$ \\
$\epsilon_{v t x}$ & $95 \pm 1.0 \%$ & - & $95 \pm 1.0 \%$ & $95.0 \pm 1.0$ \\
$\epsilon_{\text {tight }}$ & $82.5 \pm 0.5$ & $85.6 \pm 0.2$ & $96.4 \pm 1.0 \%$ & $96.4 \pm 0.5$ \\
$\epsilon_{\text {plug }}$ & $85.9 \pm 0.8$ & $91.2 \pm 0.2$ & $94.2 \pm 1.0 \%$ & $94.2 \pm 0.8$ \\
\hline$\epsilon_{\text {phoenix }}$ & N/A & N/A & $98.6 \pm 0.6$ & $98.6 \pm 0.6$ \\
\hline \multicolumn{2}{|l}{ Total Correction Factor } & & $\epsilon_{\text {Data }} / \epsilon_{M C}=81.9 \pm 1.7$ \\
\hline
\end{tabular}

Table 4.6: A summary of the efficiencies and correction factors used for the $Z_{C P}^{0}$ cross-section calculation. Details about $\epsilon_{p l u g}$ are found in [64]. The total correction factor is the product of the terms in the right column. 
For the $Z_{C C}^{0}$ candidates, it is required that the electrons are of opposite charge. This requirement is not made for the $Z_{C P}^{0}$ channel due to the two charge track hypothesis of the PHOENIX tracking algorithm, described in section 3.3.2. Due to this tracking technique, there is a non-negligible fraction of candidate plug electrons where the wrong sign track is assigned. This issue is addressed in section 5.3.2.

\section{3 $\quad Z^{0} \rightarrow \mu^{+} \mu^{-}$Selection}

The triggering of the high $P_{T}$ muon dataset is described in section 4.3.1, with a description of the data quality requirements given in section 4.3.2. The parameters used for identification are detailed in section 4.3.3, a detailed summary of the selection used for the different categories of muons is given in Table 4.10. The cuts are shown graphically in section 4.3.4, with the distributions for the different identification variables, and their efficiencies in section 4.3.5.

\subsubsection{Muon Trigger Samples}

The $Z^{0} \rightarrow \mu^{+} \mu^{-}$events are triggered by either the CMUP_18 or the CMX_18 inclusive high transverse momentum $P_{T}$ muon triggers, and referred to as $Z_{C M U P}^{0}$ and $Z_{C M X}^{0}$ events respectively.

- The level 1 trigger looks for spatial coincidences of CMU and CMP stubs with extrapolated XFT tracks.

- At level 2, stricter cuts are placed on $P_{T}^{X F T}$. 
- At level 3: the matching between XFT and muon stubs is tightened. $\triangle X_{C M U / C M P / C M X}$ is the difference in $x$ between the position of the $(\mathrm{CMU} / \mathrm{CMP} / \mathrm{CMX})$ stub and the track extrapolation to the stub, in centimetres,

The requirements of the CMUP_18 and CMX_18 triggers are summarised in tables 4.7 and 4.8. Their efficiencies are $88.7 \pm 0.9 \%$ [58] and $95.4 \pm 1.0$ $\%$ [58] respectively. Requirements are placed on the presence of a stub, the number of layers (in the muon chamber) having a hit and the $P_{T}$ of the XFT track.

\begin{tabular}{|c|c|}
\hline Trigger Level & Requirement \\
\hline 1 & $P_{T}^{C M P}>3 \mathrm{GeV} / c,>2$ layers \\
& $P_{T}^{C M U}>6 \mathrm{GeV} / c$ \\
& $P_{T}^{X F T}>4 \mathrm{GeV} / c$ \\
\hline 2 & $P_{T}^{X F T}>8 \mathrm{GeV} / c$ \\
\hline 3 & $P_{T}^{C O T}>18 \mathrm{GeV} / c$ \\
& $\Delta X_{C M P}<20 \mathrm{~cm}, \Delta X_{C M U}<10 \mathrm{~cm}$ \\
\hline
\end{tabular}

Table 4.7: CMUP_18 Trigger requirements.

\subsubsection{Good run list}

For the $Z^{0} \rightarrow \mu^{+} \mu^{-}$channel, good results from the monitoring of the CMU, CMX and CMP muon systems are required, in addition to "good" status requirements for the COT, calorimeter and showermax systems[53]. The CMX trigger was not operational in the beginning of the data taking, resulting in a reduced luminosity for the CMX dataset. The triggers, datasets and 


\begin{tabular}{|c|c|}
\hline Trigger Level & Requirement \\
\hline 1 & $\begin{array}{c}P_{T}^{C M X}>6 \mathrm{GeV} / c \\
P_{T}^{X F T}>8 \mathrm{GeV} / c\end{array}$ \\
\hline 2 & N $/ \mathrm{A}$ \\
\hline 3 & $\begin{array}{l}P_{T}^{C O T}>18 \mathrm{GeV} / c \\
\Delta X_{C M X}<10 \mathrm{~cm}\end{array}$ \\
\hline
\end{tabular}

Table 4.8: CMX_18 Trigger requirements.

luminosities for the individual analysis channels are listed in Table 4.9, after the good run requirements have been applied.

\begin{tabular}{|l|r|r|}
\hline Sample & L3 Trigger & $\mathcal{L}\left(\mathrm{pb}^{-1}\right)$ \\
\hline$Z^{0} \gamma \rightarrow \mu \mu$ CMUP & CMUP_18 & $192 \pm 12$ \\
$Z^{0} \gamma \rightarrow \mu \mu$ CMX & CMX_18 & $175 \pm 11$ \\
\hline
\end{tabular}

Table 4.9: L3 Trigger Path, Dataset name and integrated luminosity $(\mathcal{L})$ for the muon analysis channels. There is a $6 \%$ systematic error quoted on the luminosity, see section 3.3.6.

\subsubsection{Muon Identification Variables}

A muon is identified by a track matched to hits in the muon chambers, and with little associated energy in the calorimeters. The variables used for the muon identification are given below:

- $P_{T}$

Unlike electrons, muons do not deposit all their energy in the calorimeters. The $P_{T}$ of the track of the muon is used instead, with a constraint 
requiring the track to originate from the beamline. Muons are considered if they have $P_{T}>20 \mathrm{GeV} / \mathrm{c}$. To ensure a well measured track for the muon, seven hits, in at least three of the axial $(A x)$ and Stereo $(S t)$ superlayers $(S L)$ of the COT, are required.

\section{- $d_{0}$}

The impact parameter of the muons track is required to be less than $0.02(0.2) \mathrm{cm}$ for tracks with(without) SVX hits. This is to ensure the tracks originate from the interaction point, reducing cosmic ray contamination.

- $z_{0}$

The $z_{0}$ from the second muon is required to be within $4 \mathrm{~cm}$ of that of the tight muon. This is to ensure a well measured track originating from the same vertex as the first muon.

- $\rho$ cut

The radius of the point at which a particle exits the COT (exit radius), $\rho$, can be derived from the $\eta$ and $z_{0}$ of the track, and the length of the COT of $z_{C O T}=155 \mathrm{~cm}$ (shown schematically in figure 4.4$)$ :

$$
\begin{aligned}
\rho & =\frac{\operatorname{sign}(\eta) \cdot z_{C O T}-z_{0}}{\tan (\lambda)} \\
\text { where }: \lambda & =\frac{\pi}{2}-\theta \\
\text { and }: \theta & =2 \cdot \tan ^{-1}\left(e^{-\eta}\right)
\end{aligned}
$$

A cut of $\rho>140 \mathrm{~cm}$ ensures that the track traverses all 4 superlayers. This cut is required due to the limited XFT trigger acceptance at low $\rho$ due to the requirement of hits on all 4 axial superlayers. 
- Iso4

Muon isolation cuts are very effective in removing background, such as soft $\mu$ 's produced in jets. A cone of size $\Delta R=\sqrt{(\Delta \phi)^{2}+(\Delta \eta)^{2}}=0.4$ is placed about the beam constrained muon track. A limit can then be placed on the $P_{T}$ sum in the cone (Iso4), excluding the energy cluster of the muon itself. A successful muon candidate must have Iso4 $/ P_{T}<0.1$.

- Fiducial region requirement For tight CMUP(CMX) muons, the track is required to be fiducial to the $\mathrm{CMUP}(\mathrm{CMX})$ detector, i.e. when a track is extrapolated from the tracking system through the muon chambers, it must pass through all 4 chambers of the muon detector, enabling a stub to be reconstructed. Limits are placed on the fiducial distances $x$ and $z$, as defined in Figure 4.5. A track is defined as fiducial in the CMUP if:

- $x$-fiducial distance from the $\mathrm{CMU}<0 \mathrm{~cm}$

- $z$-fiducial distance from the $\mathrm{CMU}<-3 \mathrm{~cm}$

- $x$-fiducial distance from the CMP $<0 \mathrm{~cm}$

- $z$-fiducial distance from the CMP $<-3 \mathrm{~cm}$

A track is defined as fiducial in the CMX if:

- $x$-fiducial distance from the CMX $<0 \mathrm{~cm}$

- $z$-fiducial distance from the CMX $<-3 \mathrm{~cm}$

Muon candidates are rejected which have stubs reconstructed from hits in either the miniskirt or keystone regions of the detector. These sec- 


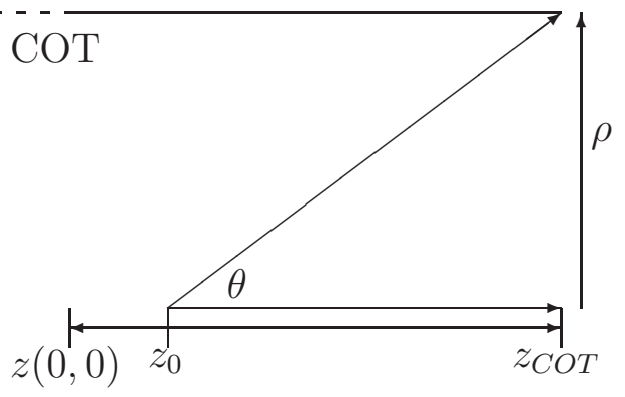

Figure 4.4: The exit radius ( $\rho$ ) of particles exiting the COT can be calculated from the $\theta$ and $z_{0}$ of the track.

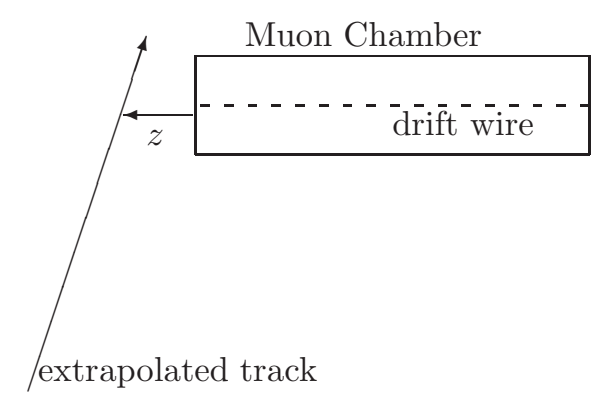

Figure 4.5: Illustration of the muon $z$ fiducial distance. The $z(x)$-fiducial distance is defined to be the distance between the extrapolated COT track and the edge of the muon chamber, in the direction parallel (perpendicular) to the drift wire. The convention is used whereby tracks which extrapolate to the outside (inside) of the chamber have fiducial distances greater (less) than 0 . 
tions of the detector, as described in section 3.3.5, were not fully operational for the entire data sample.

- stub-track matching: $\triangle X_{C M U / C M P / C M X}$

The difference in $x$ between the position of the (CMU/CMP/CMX) stub and the track extrapolation to the stub, in centimetres. If a muon has a CMUP stub, then it is required that $\left|\Delta X_{C M U}\right|<3 \mathrm{~cm}$ and $\left|\Delta X_{C M P}\right|<5 \mathrm{~cm}$. Muons with CMX stubs must have $\left|\Delta X_{C M X}\right|<$ $6.2 \mathrm{~cm}$.

- $E_{\text {had }}, E_{\text {em }}$

Muons, as minimum ionising particles, do not deposit much energy in either the hadronic or electromagnetic calorimeters. Limits can then be placed on the amount of energy observed to distinguish muons from other particles. Candidates must have $E_{e m}<2+\max (0,0.0115 *(p-$ 100)) $\mathrm{GeV}$ and $E_{\text {had }}<6+\max (0,0.028 *(p-100)) \mathrm{GeV}$, where $p$ is the total momentum of the $\mu$ candidate. The form of these functions result in the cut being effectively relaxed at $p>100$. This is to allow for the fact that higher momentum $\mu$ 's deposit more energy in the calorimeter. This parametrisation has been optimized from data from CDF run I[66]

\subsubsection{N-1 plots}

N-1 plots are made for the muon candidates. After one tight CMUP or CMX muon is selected for an event, N-1 track requirements are applied to the second muon. The distribution for the Nth variable is plotted in Figures 4.6 and 4.7 . 


\begin{tabular}{|c|c|c|c|}
\hline & Tight CMUP Muon & Tight CMX Muon & Loose Muon \\
\hline $\begin{array}{l}\text { Fiducial and } \\
\text { Kinematic Cuts: }\end{array}$ & \multicolumn{3}{|c|}{$P_{T}>20 \mathrm{GeV}$} \\
\hline $\begin{array}{l}\text { Muon Track Cuts: } \\
\text { (trkid) }\end{array}$ & \multicolumn{3}{|c|}{$\begin{array}{c}N_{A x} \mathrm{SL} \geq 3, N_{S t} \mathrm{SL} \geq 3, \text { with } \geq 7 \text { hits } \\
\left|d_{0}^{\text {corr }}\right|<0.02(0.2) \mathrm{cm} \text { for SVX }(\mathrm{COT}) \text { tracks } \\
E_{\text {em }}<2+\max (0,0.0115 *(p-100)) \mathrm{GeV} \\
E_{\text {had }}<6+\max (0,0.028 *(p-100)) \mathrm{GeV}\end{array}$} \\
\hline $\begin{array}{l}\text { Isolation Cuts: } \\
(\text { iso) }\end{array}$ & \multicolumn{3}{|c|}{$I s o 4 / P_{T}<0.1$} \\
\hline $\begin{array}{l}\text { Muon Stub } \\
\text { Cuts: } \\
(\text { rec })\end{array}$ & $\begin{array}{l}\text { CMU and CMP stub } \\
\qquad \begin{array}{r}\left|\Delta X_{C M U}\right|<3 \mathrm{~cm} \\
\left|\Delta X_{C M P}\right|<5 \mathrm{~cm} \\
\text { x-fiducial dista } \\
\text { z-fiducial distar } \\
\rho_{C O T}>\end{array}\end{array}$ & $\begin{array}{l}\left|\Delta X_{C M X}\right|<6.2 \mathrm{~cm} \\
\text { ace }<0.0 \mathrm{~cm} \\
40<<-3.0 \mathrm{~cm} \\
40 \mathrm{~cm}\end{array}$ & \\
\hline
\end{tabular}

Table 4.10: Table of cuts to select tight and loose muons. No explicit cut on the tight muon $\eta$ is made but due to the requirement of a CMU or CMX stub, this corresponds to cut on detector $|\eta|<1.0$. 
In Figure 4.6, the $P_{T}$ cut of $20 \mathrm{GeV} / c$ effectively removes a background of low $P_{T}$ muon candidates, such as soft $\mu$ 's produced in jets. Above the minimum $P_{T}$ cut, the $\mathrm{MC}$ gives a good description of the $P_{T}$ candidates from the decay of the $Z^{0}$ boson. The distribution of the fractional track isolation, Iso4 $/ P_{T}$ is similar in data and simulation. The amount of energy deposited in the electromagnetic (hadronic) calorimeter is plotted on two different scales to show the peak at $\sim 0.3(\sim 2) \mathrm{GeV}$, typical of a minimal ionisation particle with a small tail to high energies. The electromagnetic energy distribution predicted by the simulation is in good agreement with that found in data. The minimal ionisation peak in the hadronic calorimeter is however predicted to be at a higher energy $(2.2 \mathrm{GeV})$ in $\mathrm{MC}$ than in data $(1.8 \mathrm{GeV})$. This is due a problem with the $\mu$ response in the simulation which has been fixed in the latest software release. Due to the position of the cut, this slight discrepancy does not affect this analysis.

In Figure 4.7, the impact parameter, d0, is plotted for tracks with and without hits in the SVX detector. The MC simulation gives good description of these distributions. As expected, the distribution is narrower for those tracks with SVX hits, than those without. Distributions for the matching between the tracks and the CMU, CMP and CMX stubs, show larger "shoulders" in the distributions for data than that for MC, with a possible secondary peak in the $\Delta X_{C M X}$ distribution, due to miscalibration and misalignment issues which have since been fixed in the latest software releases. 

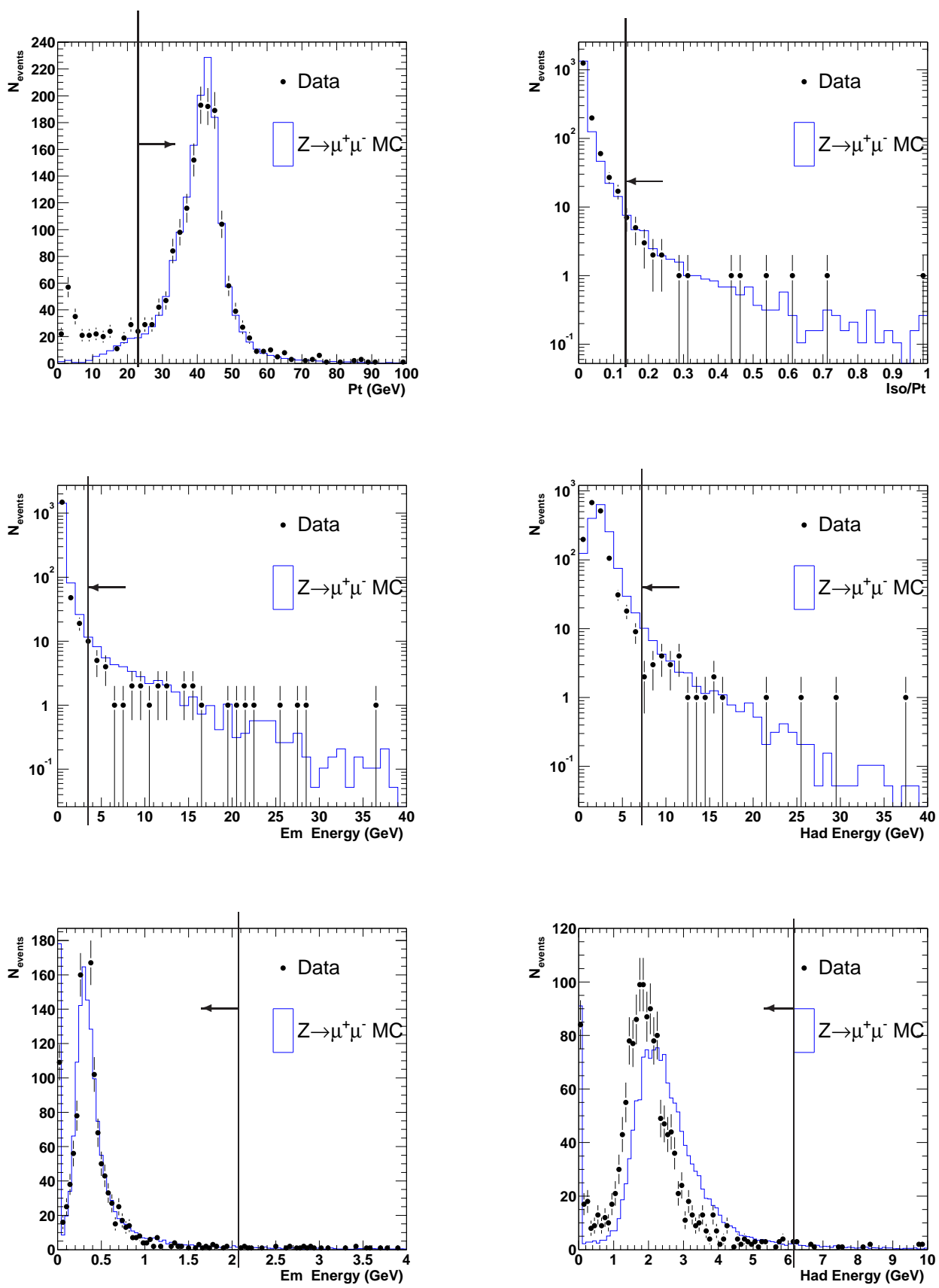

Figure 4.6: N-1 distributions for muon identification variables used for the selection of the events, plotted for data (dots) and MC (solid histogram). The arrow shows graphically the selection cut. The MC simulation is normalised to the luminosity of the data. 

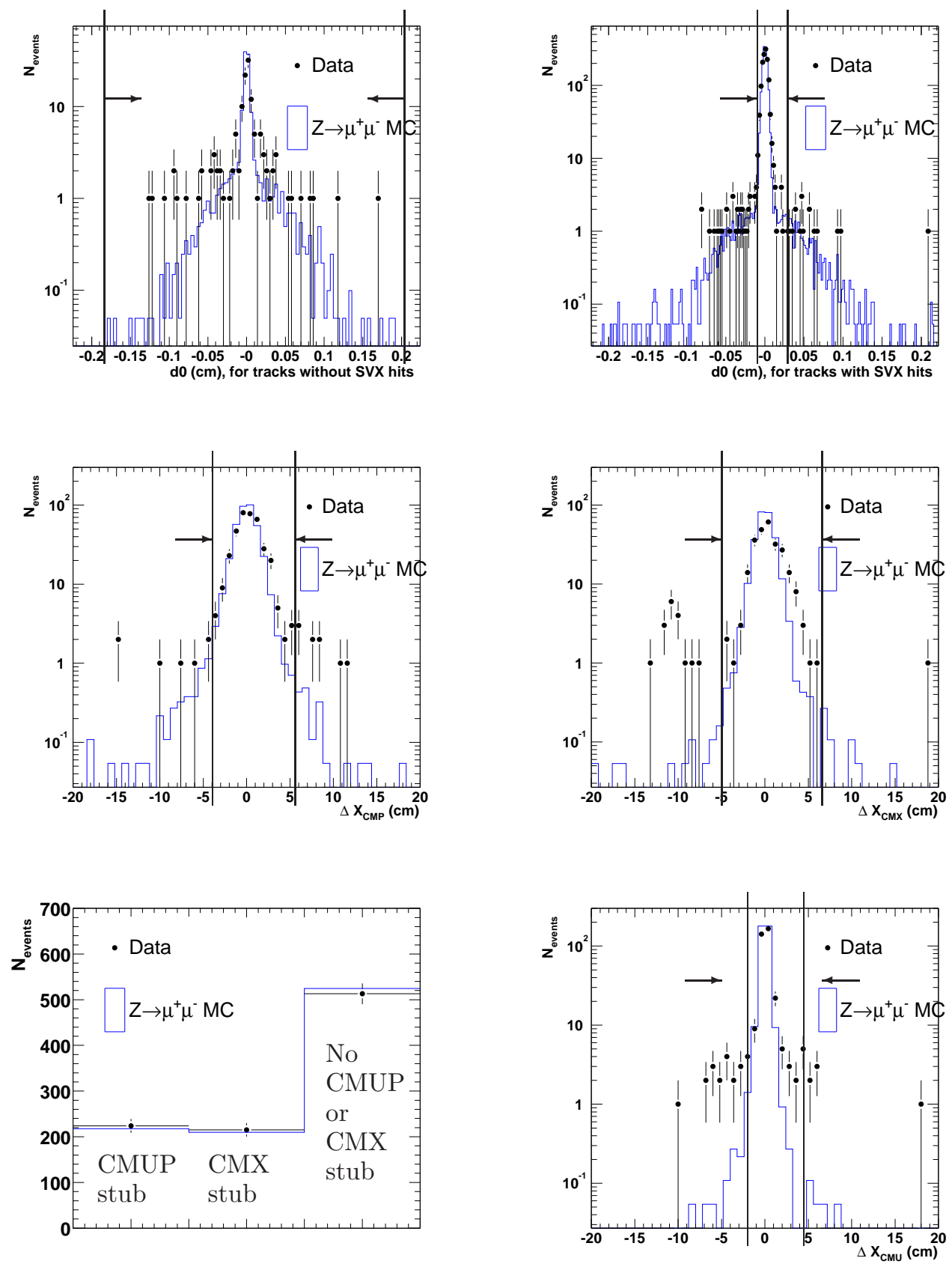

Figure 4.7: N-1 distributions for muon identification variables used for the selection of the events, plotted for data (dots) and MC (solid histogram). The arrow shows graphically the selection cut. The MC simulation is normalised to the luminosity of the data. 


\subsubsection{Muon Efficiency}

Muon selection efficiency is determined from Monte Carlo simulation. Correction factors have been calculated to allow for imperfect simulation, in an analogous fashion to section 4.2.5. The correction factors for the CMUP and CMX muons are summarised in tables 4.11 and 4.12 respectively. A break down of the individual cut efficiencies is shown in Appendix A.1.

- The efficiency of the $z_{0}$ requirement is $95 \% \pm 0.4 \%\left(\epsilon_{z 0}\right)$, measured in data[59]. Only events with $\left|z_{0}\right|<60 \mathrm{~cm}$ are generated in Monte Carlo.

- The trigger efficiencies $\left(\epsilon_{\text {trg }}\right)$ are 0.887 and 0.954 respectively for CMUP and CMX muons respectively (see section 4.3.1).

- There is good agreement between the data and MC COT tracking efficiencies $\left(\epsilon_{t r k}\right)$. The correction factor is $1.000 \pm 0.004[62]$.

- The efficiency for a muon to pass the identification criteria in Table 4.10 is separated into three terms.

- The efficiency correction for a muon in the CMUP+track (CMX+track) sample to pass the track quality and calorimeter cuts $\left(\epsilon_{\text {trkid }}\right)$, is $99.3 \pm 0.5 \%(101.8 \pm 0.7 \%)[58,62]$.

- The ratio of muon isolation requirement $\left(\epsilon_{i s o}\right)$ between data and $\mathrm{MC}$ is $1.002 \pm 0.003$ for the CMUP and $0.999 \pm 0.004$ for the CMX muons[58, 62].

- The efficiency for a muon track to be linked to a reconstructed CMUP (CMX) muon stub, $\epsilon_{\text {stubid }}$, is $89.0 \pm 0.8 \%(98.8 \pm 0.4 \%)$ $[58,62]$. 
- The efficiency for a real $Z \rightarrow \mu \mu$ not to be tagged as a cosmic event $\left(\epsilon_{\text {cos }}\right)$ is $0.9994 \pm 0.006$ [62], see section 5.3.1.

\begin{tabular}{|l|c|c|c|}
\hline Efficiency & $\epsilon_{\text {Data }}$ & $\epsilon_{M C}$ & $\left(\epsilon_{\text {Data }} / \epsilon_{M C}\right)^{l}$ \\
$(\%)$ & $(\%)$ & $(\%)$ \\
\hline$\epsilon_{\text {trg }}$ & $88.7 \pm 1.0$ & - & $88.7 \pm 0.9$ \\
$\epsilon_{z 0}$ & $95.0 \pm 0.4$ & - & $95.0 \pm 0.4$ \\
$\epsilon_{\text {trk }}$ & $99.6 \pm 0.4$ & $99.6 \pm 0.2$ & $100.0 \pm 0.4$ \\
$\epsilon_{\text {trkid }}$ & $92.5 \pm 0.5$ & $93.2 \pm 0.2$ & $99.3 \pm 0.5$ \\
$\epsilon_{\text {iso }}$ & $98.0 \pm 0.3$ & $97.9 \pm 0.1$ & $100.2 \pm 0.3$ \\
$\epsilon_{\text {stubid }}$ & $85.2 \pm 0.8$ & $95.7 \pm 0.1$ & $89.0 \pm 0.8$ \\
$\epsilon_{\text {cos }}$ & $99.94 \pm 0.6$ & - & $99.94 \pm 0.06$ \\
\hline
\end{tabular}

Table 4.11: A summary of the efficiencies, in data and MC, and correction factors, $\left(\epsilon_{\text {Data }} / \epsilon_{M C}\right)^{l}$, used for the $Z_{C M U P}^{0}$ cross-section calculation.

\subsection{6 $\quad \mathrm{Z}_{\mathrm{CMUP}}^{0}$ and $\mathrm{Z}_{\mathrm{CMX}}^{0}$ Selection Efficiencies}

A $Z_{C M U P}^{0}\left(Z_{C M X}^{0}\right)$ candidate consists of a tight CMUP(CMX) muon and a loose muon candidate. These are not mutually exclusive samples since the loose muon in the $Z_{C M U P}^{0}$ sample could pass the tight CMX muon selection. A $Z_{C M U P / X}^{0}$ candidate is defined to be a either a $Z_{C M U P}^{0}$ or a $Z_{C M X}^{0}$ candidate. From $Z^{0} \rightarrow \mu^{+} \mu^{-}$MC, $67.8 \pm 0.4 \%$ of muons which points towards an active region of the detector, point to the CMUP, and $32.2 \pm 0.4 \%$ point to the CMX. Using these fractions, the efficiencies for CMUP/X muons can be calculated:

$$
\epsilon^{C M U P / X}=0.678 \epsilon^{C M U P}+0.322 \epsilon^{C M X} .
$$




\begin{tabular}{|l|c|c|c|}
\hline Efficiency & $\epsilon_{\text {Data }}$ & $\epsilon_{M C}$ & $\left(\epsilon_{\text {Data }} / \epsilon_{M C}\right)^{l}$ \\
& $(\%)$ & $(\%)$ & $(\%)$ \\
\hline$\epsilon_{\text {trg }}$ & $95.4 \pm 1$ & - & $95.4 \pm 1.0$ \\
$\epsilon_{z 0}$ & $95.0 \pm 1.0$ & - & $95.0 \pm 1.0$ \\
$\epsilon_{\text {trk }}$ & $99.6 \pm 0.4$ & $99.6 \pm 0.2$ & $100 \pm 0.4$ \\
$\epsilon_{\text {trkid }}$ & $92.4 \pm 0.7$ & $90.8 \pm 0.3$ & $101.8 \pm 0.7$ \\
$\epsilon_{\text {iso }}$ & $97.6 \pm 0.4$ & $97.7 \pm 0.1$ & $99.9 \pm 0.4$ \\
$\epsilon_{\text {stubid }}$ & $98.6 \pm 0.3$ & $99.7 \pm 0.1$ & $98.8 \pm 0.4$ \\
$\epsilon_{\text {cos }}$ & $99.94 \pm 0.6$ & - & $99.94 \pm 0.06$ \\
\hline
\end{tabular}

Table 4.12: A summary of the efficiencies, in data and MC, and correction factors, $\left(\epsilon_{\text {Data }} / \epsilon_{M C}\right)^{l}$, used for the $Z_{C M X}^{0}$ cross-section calculation.

The efficiencies associated with CMUP/X muons are given in table 4.13.

When these efficiency corrections of section 4.3.5 are applied, consideration must be taken of the fact that each $\mathrm{Z}$ boson candidate has two tracks. Complications arise in the muon channel for the fraction of events where both muon tracks point towards the active regions of the detector. For these events, either muon can satisfy the stub criteria. The form of this correction factor can be simplified [62] by assuming that the trigger muon is also the "tight muon" which passes the stub requirement. This assumption is correct to better than $0.1 \%[67]^{3}$.

$$
\begin{aligned}
\epsilon_{\text {tot }}= & \epsilon_{z 0} \times \epsilon_{\text {cos }} \times \epsilon_{\text {trk }}^{2} \times \epsilon_{\text {iso }}^{2} \times \\
& \epsilon_{\text {stubid }} \times \epsilon_{\text {trg }} \times \epsilon_{\text {trkid }} \times\left(1+f_{\text {dd }} \times\left(1-\epsilon_{\text {stubid }} \times \epsilon_{\text {trg }} \times \epsilon_{\text {trkid }}\right)\right)
\end{aligned}
$$

\footnotetext{
${ }^{3}$ This value was determined from data, from a different software release which allowed the "identity" of the trigger muon to be known. This was not the case for the software release used in this analysis.
} 


\begin{tabular}{|l|c|}
\hline Efficiency & $\begin{array}{c}\left(\epsilon_{\text {Data }} / \epsilon_{M C}\right)^{l} \\
(\%)\end{array}$ \\
\hline$\epsilon_{\text {trg }}$ & $90.1 \pm 1.0$ \\
$\epsilon_{z 0}$ & $95.0 \pm 1.0$ \\
$\epsilon_{\text {trk }}$ & $100.0 \pm 0.4$ \\
$\epsilon_{\text {trkid }}$ & $100.1 \pm 0.6$ \\
$\epsilon_{\text {iso }}$ & $100.1 \pm 0.4$ \\
$\epsilon_{\text {stubid }}$ & $92.2 \pm 0.7$ \\
$\epsilon_{\text {cos }}$ & $99.94 \pm 0.06$ \\
\hline
\end{tabular}

Table 4.13: A summary of the efficiency correction used for the $Z_{C M U P / X}^{0}$ cross-section calculation.

The total muon efficiency correction is given by equation 4.7. The efficiency for a real $Z \rightarrow \mu \mu$ not to be tagged as a cosmic event $\left(\epsilon_{c o s}\right)$ and the requirement on the z-vertex $\left(\epsilon_{z 0}\right)$ enter linearly as they are applied on a per event basis. The track reconstrution $\left(\epsilon_{t r k}\right)$ and the isolation efficiency $\left(\epsilon_{i s o}\right)$ are applied to both muon tracks and hence these terms appear squared in the total efficiency calculation. The muon stub id $\epsilon_{\text {stubid }}$ and trigger efficiencies $\epsilon_{t r g}$ appear linearly but an extra correction factor is required to account for the fraction of events $f_{d d}$ where both muons point at the active regions of the muon detectors. The track id efficiency has been measured in two different $\eta$ ranges with CMUP and CMX muons. The loose muon has the same $\eta$ range as a CMUP/X muon and hence takes the same value of $\epsilon_{\text {trkid }}$ of $100.1 \pm 0.6 \%$. Therefore to simplify the total muon efficiency correction, $\epsilon_{\text {trkid }}$ is only applied to the tight muon. It is entered linearly with the correction factor $f_{d d}$. 
The fraction $f_{d d}$ is determined from Monte Carlo. This fraction for $Z_{C M U P}^{0}, Z_{C M X}^{0}$ and $Z_{C M U P / X}^{0}$ (the combined $Z_{C M U P}^{0}$ and $\left.Z_{C M X}^{0}\right)$ samples are $0.255 \pm 0.002,0.118 \pm 0.002$ and $0.389 \pm 0.002$ respectively. The resultant total efficiencies, $\epsilon_{t o t}$, calculated by equation 4.7 are $78.7 \pm 1.8,91.3 \pm 1.8$ and $84.7 \pm 2.0$.

\subsection{7 $\quad Z^{0}$ selection}

$Z^{0} \rightarrow \mu^{+} \mu^{-}$events are selected by requiring one "tight" muon, which passes all the muon identification cuts, and one "loose" muon, which passes all the cuts except the stub requirements. The muon identification cuts are detailed in Table 4.10. The two muons are required to be of opposite charge. For the muon channel, a muon is categorised as a CMUP muon if a muon stub is found in both the CMU and CMP detectors. A muon stub found in the CMX detector is referred to as a CMX muon. Events are categorised as CMUP + track $\left(Z_{C M U P}^{0}\right)$ and CMX + track $\left(Z_{C M X}^{0}\right)$, where stub requirements are made on one muon, and only track requirements are made for the second muon. The invariant mass of both must be in the range $40<m(l, l)<$ $130 \mathrm{GeV}$. This mass range ensures that the lepton pair are consistent with the decay of a $Z^{0}$ boson. In addition, the lower cut reduces the Drell-Yan contribution. 


\section{Chapter 5}

\section{Inclusive $\mathrm{Z}^{0}$ cross-section}

\section{calculation}

In this section the measurement of the inclusive cross-section is described. Section 5.1 describes how the inclusive $Z^{0}$ sample is used as a tool to calibrate the calorimeter energy scale. The acceptancexefficiency calculation is given in section 5.2 and an estimation for the background to the $Z^{0}$ sample is given in section 5.3. The inclusive $Z^{0}$ production, calculated using this sample of events with an invariant mass range between 66 and $116 \mathrm{GeV} / c^{2}$ is compared to published CDF results[69].

\subsection{Calibrations}

In order to calibrate the energy response of the calorimeter, both in data and simulation, the reconstructed $Z^{0}$ mass was compared to the LEP value of $m_{Z}=91.1876 \pm 0.0021 \mathrm{GeV} / \mathrm{c}^{2}[2]$. The energy of the electrons and photons in the MC was scaled by 0.996, to improve the agreement to data. 
The invariant mass peaks of the $Z_{C C}^{0}, Z_{C P}^{0}, Z_{C M U P}^{0}$ and $Z_{C M X}^{0}$ candidates are shown in figures $5.1,5.2,5.3$ and 5.4 respectively.

The east and west plug calorimeters were calibrated separately. It was discovered that due to a reduction in the gain of the photomultiplier tubes of the plug electromagnetic calorimeter[68], the reconstructed $Z_{C P}^{0}$ mass fell as a function of time. In order to correct for this, a time dependent scaling factor was applied. This scaling factor is shown in table 5.1. The resulting invariant mass for the $Z_{C M U P}^{0}, Z_{C M X}^{0}, Z_{C C}^{0}$ and $Z_{C P}^{0}$ channels is shown to be flat as a function of the integrated luminosity of the $Z_{C C}^{0}$ data sample, as seen in figures 5.7, 5.8, 5.5 and 5.6 respectively.

\begin{tabular}{|l|l|l|l|}
\hline & \multicolumn{3}{|c|}{ Integrated Luminosity, $\mathrm{pb}^{-1}$} \\
Plug & $\int \mathcal{L} d t<81$ & $81<\int \mathcal{L} d t<133$ & $133<\int \mathcal{L} d t<202$ \\
\hline East & 1.091 & 1.099 & 1.113 \\
West & 1.052 & 1.087 & 1.118 \\
\hline
\end{tabular}

Table 5.1: Run dependent energy calibrations for the east and west plugs. There is a $0.3 \%$ error on the calibrations used, determined from the calibration of the data to the PDG value of the $Z^{0}$ boson mass.

\subsection{Inclusive Acceptance and $\mathrm{Z}^{0}$ Identifica- tion Efficiencies}

The product of the acceptance and efficiency is calculated in MC. An efficiency correction is applied to adjust the efficiency values up/down to their values in data as explained in chapter 4 . 


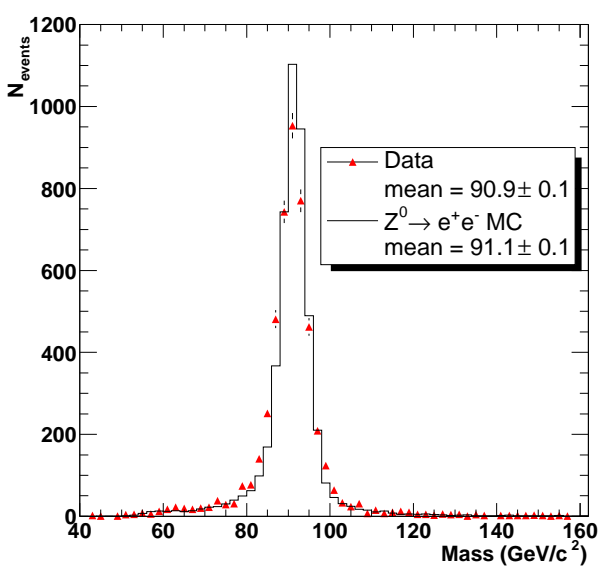

Figure 5.1: Comparison of the invariant mass of $Z_{C C}^{0}$ candidate events in the data to $Z^{0} \rightarrow e^{+} e^{-}$ MC.

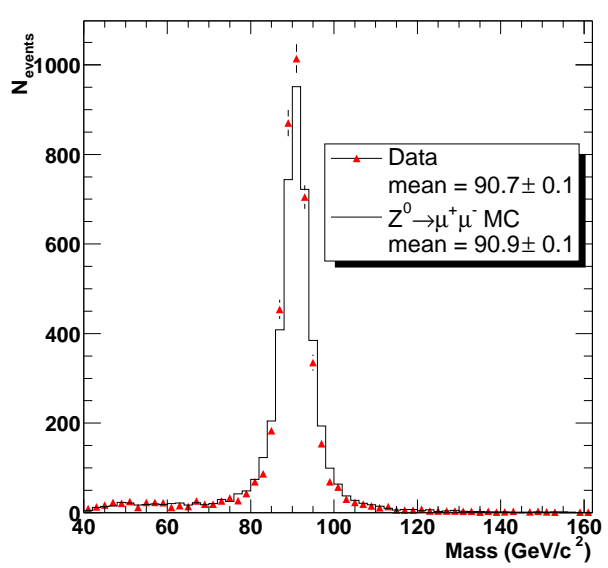

Figure 5.3: Comparison of the invariant mass of $Z_{C M U P}^{0}$ candidate events in the data to $Z^{0} \rightarrow \mu^{+} \mu^{-}$ $\mathrm{MC}$.

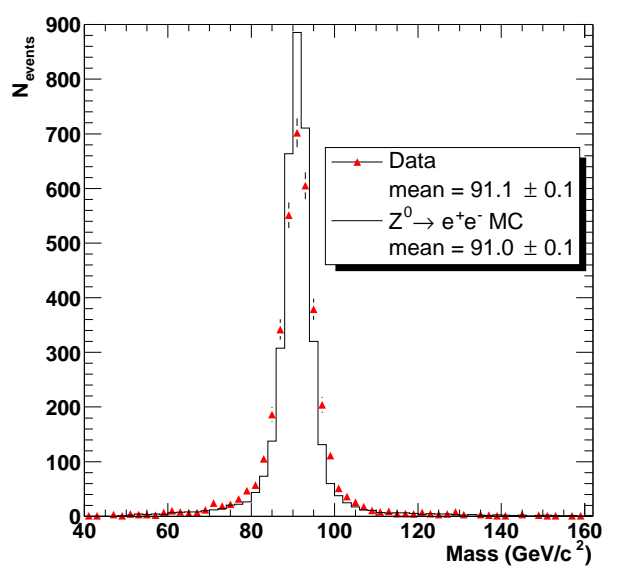

Figure 5.2: Comparison of the invariant mass of $Z_{C P}^{0}$ candidate events in the data to $Z^{0} \rightarrow e^{+} e^{-}$ MC.

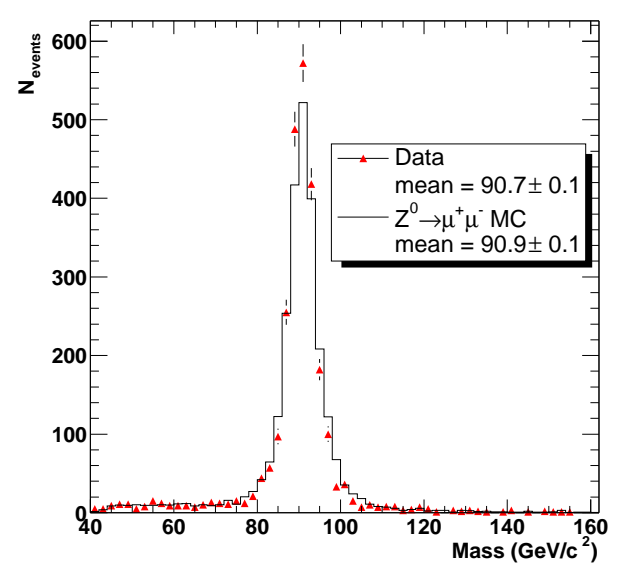

Figure 5.4: Comparison of the invariant mass of $Z_{C M X}^{0}$ candidate events in the data to $Z^{0} \rightarrow \mu^{+} \mu^{-}$ $\mathrm{MC}$. 


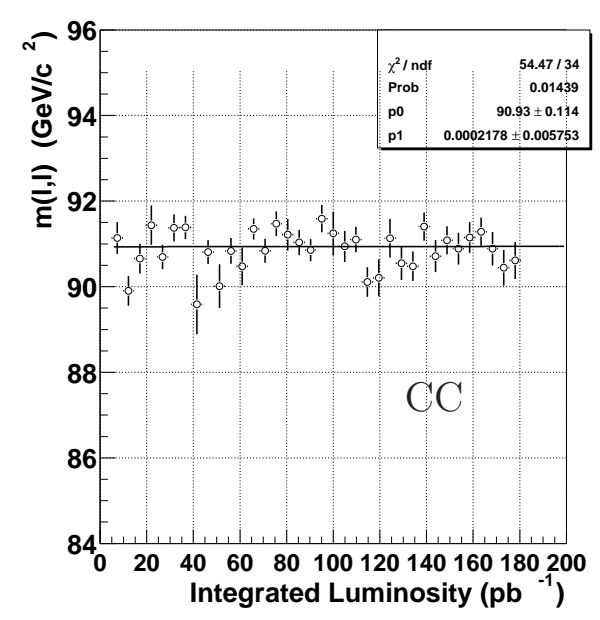

Figure 5.5: The invariant mass of $Z_{C C}^{0}$ candidate events as a function of the integrated luminosity.

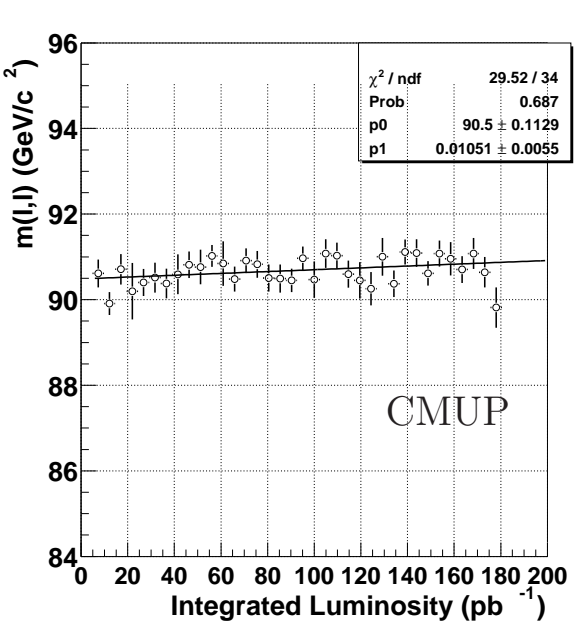

Figure 5.7: The invariant mass of $Z_{C M U P}^{0}$ candidate events as a function of the integrated luminosity of the $Z_{C C}^{0}$ sample.

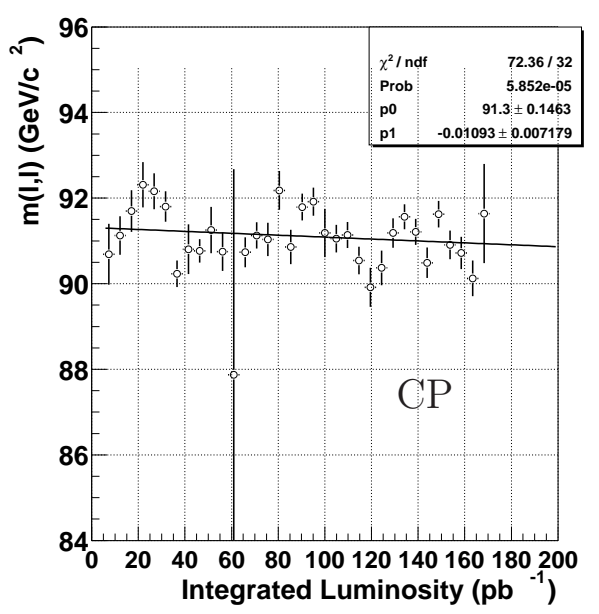

Figure 5.6: The invariant mass of $Z_{C P}^{0}$ candidate events as a function of the integrated luminosity of the $Z_{C C}^{0}$ sample.

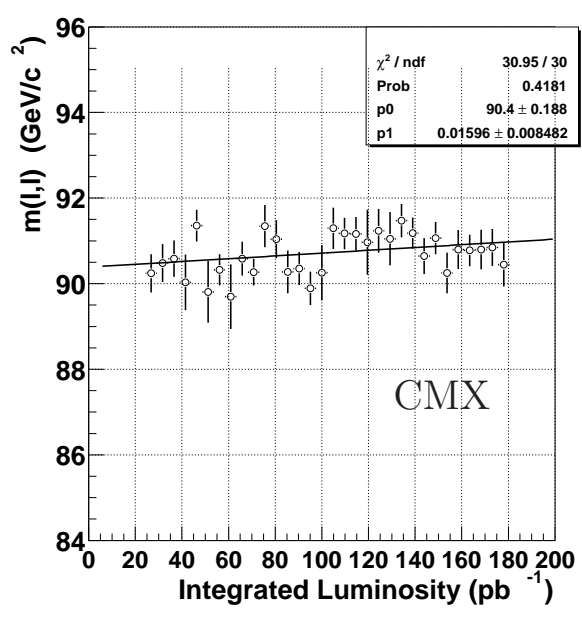

Figure 5.8: The invariant mass of $Z_{C M X}^{0}$ candidate events as a function of the integrated luminosity of the $Z_{C C}^{0}$ sample. 
In order to correctly calculate the acceptance for the cross-section value in this mass range an additional requirement is placed on the denominator of the acceptance calculation. It is required that events constituting the denominator have a generated mass of $66<m^{\text {gen }}(l, l)<116$. This ensures that the acceptance values are based only on the subset of events in this invariant mass range. The acceptance calculation for the lepton selection cuts described in sections 4.2 and 4.3 , for the inclusive $Z^{0}$ analysis, is given in tables 5.2 and 5.3 , where $m^{r e c}(l, l)$ is the reconstructed mass of the dilepton pair.

\begin{tabular}{|l|c|c|}
\hline \multirow{1}{*}{} & \multicolumn{2}{|c|}{$Z^{0} \rightarrow e^{+} e^{-}$} \\
& $\mathrm{CC}$ & $\mathrm{CP}$ \\
& $(\%)$ & $(\%)$ \\
\hline 1 Tight Lepton & $43.3 \pm 0.2$ & $43.3 \pm 0.2$ \\
1 Tight, 1 Loose lepton & $10.91 \pm 0.08$ & $13.25 \pm 0.08$ \\
Opposite Charge & $10.82 \pm 0.08$ & $\mathrm{~N} / \mathrm{A}$ \\
$66<m^{r e c}(l, l)<116 \mathrm{GeV} / \mathrm{c}^{2}$ & $10.28 \pm 0.07$ & $10.4 \pm 0.08$ \\
\hline
\end{tabular}

Table 5.2: Acceptance $\times$ efficiency for the $Z^{0} \rightarrow e^{+} e^{-}$analyses.

\begin{tabular}{|l|c|c|c|}
\hline & \multicolumn{3}{|c|}{$Z^{0} \rightarrow \mu^{+} \mu^{-}$} \\
& CMUP & CMX & CMUP $/ X$ \\
& $(\%)$ & $(\%)$ & $(\%)$ \\
\hline 1 Tight Lepton & $28.4 \pm 0.1$ & $14.3 \pm 0.1$ & $37.7 \pm 0.1$ \\
1 Tight, 1 Loose lepton & $12.02 \pm 0.07$ & $6.37 \pm 0.06$ & $14.7 \pm 0.1$ \\
Opposite Charge & $11.88 \pm 0.07$ & $6.29 \pm 0.06$ & $14.7 \pm 0.1$ \\
$66<M^{\text {rec }}(l, l)<116 \mathrm{GeV} / \mathrm{c}^{2}$ & $11.21 \pm 0.07$ & $5.96 \pm 0.05$ & $14.7 \pm 0.1$ \\
\hline
\end{tabular}

Table 5.3: Acceptance $\times$ efficiency for the $Z^{0} \rightarrow \mu^{+} \mu^{-}$analyses. 


\subsection{Background Processes}

\subsubsection{Cosmic Rejection}

Cosmic ray muons passing through the detector can mimic a $Z^{0} \rightarrow \mu^{+} \mu^{-}$ event. The standard "Cosmic Ray Tagger" algorithm[92] is used to identify these events and remove them from the $Z_{C M U P}^{0}$ and $Z_{C M X}^{0}$ samples:

- Timing information from the hadron calorimeter TDC and from the TOF detector provide some discriminating power. A pair of muons produced at the beamline will reach these detectors at approximately the same time. A cosmic ray travelling downwards, will hit one set of detectors before passing through the COT, and another set afterwards, as the cosmic ray passes out of CDF.

- The back-to-back topology and the correlation with the $r \phi$ impact parameter of the two reconstructed tracks are also used.

Occasionally a genuine $Z^{0} \rightarrow \mu^{+} \mu^{-}$event may be misidentified as a cosmic event and excluded. The resulting efficiency for a $Z^{0}$ to pass the cosmic tagger, $\epsilon_{\text {cos }}$, has been measured [62] to be $\epsilon_{\text {cos }}=99.94 \pm 0.06 \%$.

\subsection{2 $\quad Z^{0}$ Fake Background}

The most significant source of background are QCD events where one or both of the reconstructed leptons originate from jets, which produce both real and fake leptons. In this case, the charge of the reconstructed leptons is expected to be uncorrelated. Therefore the background contribution to our signal, of a pair of oppositely signed leptons, can be estimated from the number of 
events with a pair of leptons which pass the selection criteria as detailed in chapter 4 , but are of the same charge, i.e. "same-sign" events.

This method is employed to estimate the background contribution to the inclusive $Z_{C C}^{0}, Z_{C M U P}^{0}$ and $Z_{C M X}^{0}$ samples.

For the $Z_{C C}^{0}$, same-sign events can be caused by "tridents". This is where one electron from the decay of a $Z^{0}$ boson emits a bremsstrahlung photon, which subsequently convert into an electron positron pair. If such an event occurs, the sign of the electron can be mis-identified. The number of trident events is estimated by counting the number of same-sign events in $Z^{0} \rightarrow e^{+} e^{-}$ Monte-Carlo. Therefore the background in the $Z_{C C}^{0}$ sample is given by the number of same-sign events in data, minus the number of same-sign events in Monte-Carlo, scaled to the same luminosity.

No same-sign events are found for the $Z_{C M U P}^{0}$ and $Z_{C M X}^{0}$ samples. For the $Z_{C C}^{0}$ sample, there are 71 same-sign events in the data sample, and 28.2 events in the $Z^{0} \rightarrow e^{+} e^{-}$Monte-Carlo sample, scaled to the luminosity of the data, giving a background prediction of $43 \pm 4$ events.

A different method must be used for the $Z_{C P}^{0}$ sample, where no requirement is made on the charges of the lepton pair. The rate at which a jet fakes either a tight central electron or plug electron (excluding PHOENIX track requirement) has been developed elsewhere[112, 68], with a systematic error of $30 \%$. These rates are shown in figures 5.9 and 5.10 respectively. If a central jet has $E_{T}>25 \mathrm{GeV}$ and a plug jet has $E_{T}>20 \mathrm{GeV}$, their mass is entered in a dijet mass distribution with weight equal to the product of the fake rates for the two legs using the parameterisations shown in the two figures. One additional factor is required. The plug fake rate applied was 
developed for plug identification criteria which did not include the PHOENIX track requirement.

Using $Z^{0} \rightarrow e^{+} e^{-}$Monte-Carlo, $20 \pm 5 \%$ of plug electrons which passed all the calorimeter based plug identification criteria in table 4.4, also passed the PHOENIX track requirement. Applying the fake rates and the $20 \%$ correction, the background prediction is $11 \pm 4$ events.

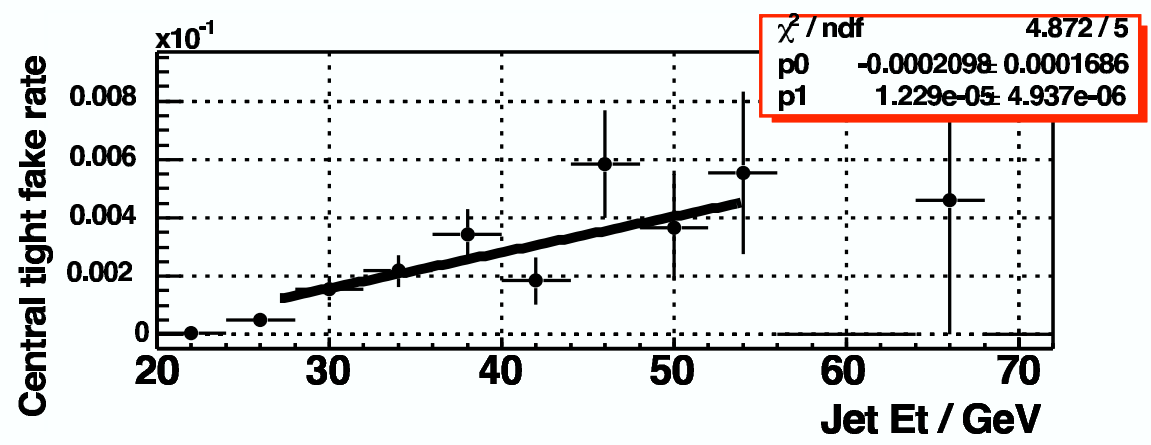

Figure 5.9: Fake Rate for a jet to fake a central tight electron. Shown is a fit to the data.

\subsection{Inclusive $Z^{0}$ and Cross-Sections}

For the $Z^{0} \gamma$ analysis the requirement is made that the invariant mass of the two leptons is between 40 and $130 \mathrm{GeV} / \mathrm{c}^{2}$. This makes the analysis sensitive to radiative Drell-Yan terms. However, in order to compare to published theoretical and experimental results, the inclusive $\mathrm{Z}$ cross section is measured in the mass range $66<m(l, l)<116 \mathrm{GeV} / \mathrm{c}^{2}$. The cross-section 

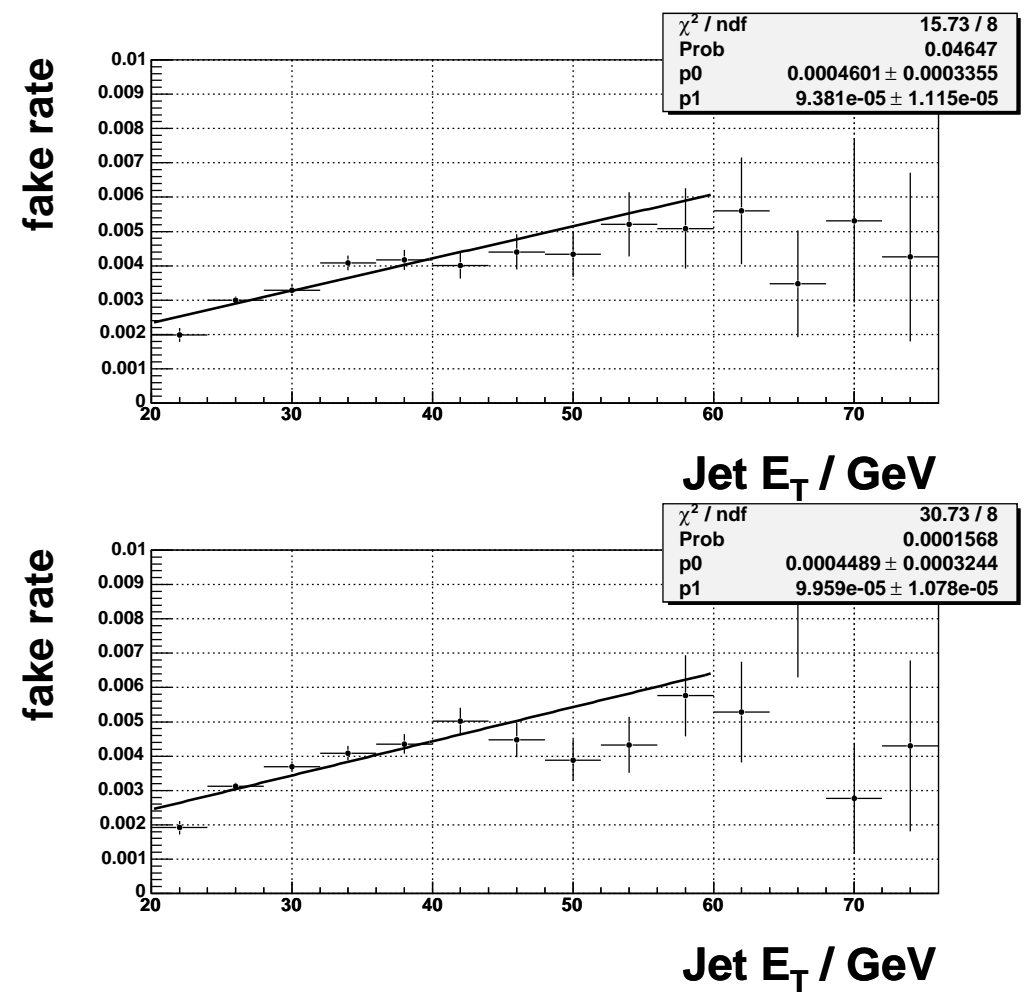

Figure 5.10: Fake Rate for a jet to fake a plug electron for the east (top plot) and west plugs (bottom plot). Shown are the fits to the data. 
is calculated from the following equation:

$$
\sigma=\frac{N_{c a n d}-N_{b k g}}{\left(A \times \epsilon_{M C}\right) \times \frac{\epsilon_{\text {Data }}}{\epsilon_{M C}} \times \epsilon_{t r g} \times \mathcal{L}}
$$

Where $N_{\text {cand }}$ is the number of candidate events, $N_{b k g}$ is the estimated background contribution, $\frac{\epsilon_{\text {Data }}}{\epsilon_{M C}}$ is the ratio of the efficiency of the selection cuts in data to that in Monte Carlo and $\epsilon_{\operatorname{trg}}$ is the efficiency of the event trigger used. The acceptance is denoted as $A$ and $\mathcal{L}$ is the integrated luminosity of the data sample analysed. The values used for the different terms are summarised in table 5.4. Details of the efficiency scale factors and acceptance numbers can be found in sections 4 and 5.2 respectively. The estimation of $N_{b k g}$ is given in section 5.3

In order to correct for the limited luminosity available for the $Z_{C M X}^{0}$ channel for the $Z_{C M U P X}^{0}$ measurement, the CMX detectors were "turned off" for a corresponding proportion of luminosity in the $Z^{0} \rightarrow \mu^{+} \mu^{-} \mathrm{MC}$.

\begin{tabular}{|c|c|c|l|c|c|c|}
\hline & $A \times \epsilon_{M C}$ & $\frac{\epsilon_{\text {Data }}}{\epsilon_{M C}}$ & $\epsilon_{\text {trg }}$ & $\mathcal{L}$ & $N_{\text {cand }}$ & $N_{\text {bkg }}$ \\
\hline$Z_{C C}^{0}$ & $0.103(<1 \%)$ & $0.907(2 \%)$ & $0.999(<1 \%)$ & $202(6 \%)$ & 4631 & $43(10 \%)$ \\
$Z_{C P}^{0}$ & $0.104(<1 \%)$ & $0.851(2 \%)$ & $0.963(<1 \%)$ & $168(6 \%)$ & 3569 & $11(30 \%)$ \\
$Z_{C M U P}^{0}$ & $0.112(<1 \%)$ & $0.787(2 \%)$ & $192(6 \%)$ & 4308 & 0 \\
$Z_{C M X}^{0}$ & $0.0596(<1 \%)$ & $0.913(2 \%)$ & $175(6 \%)$ & 2434 & 0 \\
$Z_{C M U P / X}^{0}$ & $0.147(<1 \%)$ & $0.847(2 \%)$ & $192(6 \%)$ & 6022 & 0 \\
\hline
\end{tabular}

Table 5.4: Summary table of values used for inclusive cross-section measurement. The errors on the various quantities are given as a percentage in brackets

The values in table 5.4 are inserted into equation 5.1 to obtain the crosssection measurements given in table 5.5. These values are in good agreement 


\begin{tabular}{|c|c|c|c|c|}
\hline & $\sigma(p b)$ & Stat & Sys. & Lum \\
\hline$Z_{C C}^{0}$ & 244 & \pm 4 & \pm 9 & \pm 15 \\
$Z_{C P}^{0}$ & 250 & \pm 4 & \pm 9 & \pm 15 \\
$Z_{C M U P}^{0}$ & 255 & \pm 4 & \pm 9 & \pm 15 \\
$Z_{C M X}^{0}$ & 255 & \pm 5 & \pm 9 & \pm 15 \\
\hline$Z^{0} \rightarrow e^{+} e^{-}$ & 246 & \pm 3 & \pm 10 & \pm 15 \\
$Z^{0} \rightarrow \mu^{+} \mu^{-}$ & 252 & \pm 3 & \pm 9 & \pm 15 \\
\hline
\end{tabular}

Table 5.5: The measured $\sigma\left(Z^{0}\right) \times \mathrm{BR}\left(\mathrm{l}^{+} \mathrm{l}^{-}\right)$

with the SM prediction and other analyses from CDF and D0 providing confidence in the lepton selection, $Z^{0}$ selection efficiencies and the analysis. 


\section{Chapter 6}

\section{Photon Identification}

In chapter 4 , the selection for the $Z^{0}$ bosons was described. To select $Z^{0} \gamma$ candidate events, an additional requirement is made that there is a photon with $E_{T}^{\gamma}>7 \mathrm{GeV}$, which is separated from both leptons by $\Delta R_{l \gamma}>0.7$. The photon selection criteria are detailed in section 6.1. The efficiency calculation for the photon selection is given in section 6.2 and the acceptance calculation for the $Z^{0} \gamma$ selection is given in 6.3 .

\subsection{Photon Selection}

The variables used for identifying photons are detailed in section 6.1.1 and a detailed summary of the cuts is given in table 6.1. The distributions for the different identification variables are shown in section 6.1.2. 


\subsubsection{Photon Identification Variables}

Photons and Electrons leave almost identical signals ${ }^{1}$ in the calorimeter systems of CDF. The same clustering algorithm for electromagnetic energy clusters and the same definitions for $E$ and $E_{T}$ are used for photons and electrons, as described in section 4.2.3. The obvious distinguishing feature between photons and electrons is that photons will not leave a track in the tracking chambers. The variables used for photon identification are given below.

- $E_{\text {had }} / E_{\text {em }}$

A photon produced at the centre of the detector will deposit most of its energy in the electromagnetic calorimeter with only a small amount in the hadronic calorimeter. The ratio of the hadronic to electromagnetic energy $\left(E_{\text {had }} / E_{\text {em }}\right)$, is required to be less than $<0.125$.

- $\chi_{\text {strip }}^{2}\left(\chi_{w i r e}^{2}\right)$

The comparison of the CES shower profile in the strip(wire) view, to the same profile extracted from test beam electrons, is used to distinguish between electromagnetic showers from prompt photon and electrons and those from other particles, e.g. $\pi^{0} \rightarrow \gamma \gamma$, where both $\gamma$ s produce an energy cluster. A $\chi^{2}$ is formed between the data and the test beam electrons. The variable is discussed in detail in section 7.4.1. Photon candidates have $\operatorname{CES}\left(\chi_{\text {wire }}^{2}+\chi_{\text {strip }}^{2}\right) / 2<20$.

- Second CES cluster cut

A major source of background to prompt photons are neutral mesons

\footnotetext{
${ }^{1}$ the electromagnetic shower in the calorimeter will start slightly later for photons than for electrons.
} 
which decay into two photons. These should produce two CES clusters compared to the single CES cluster produced by a prompt photon. In order to remove this background, a maximum energy cut is placed on any secondary CES clusters found of $0.14 \times E_{T}$ for $E_{T}<18$, or $2.4+0.01 E_{T}$ for $E_{T}>18 \mathrm{GeV}$.

- Fiducial region requirement

The photon candidates are required to be in the central region of the CDF detector, $|\eta| \leq 1.1$, to ensure they pass through enough of the tracking volume that a track would be detected if it were actually an electron instead. Photon candidates must also be in fiducial volume with respect to the CES detectors, with CES $|x|<21.0 \mathrm{~cm}$ and $9.0<$ CES $|z|<230.0 \mathrm{~cm}$.

- Number of Tracks

The number of tracks $\left(N_{\text {track }}\right)$, reconstructed using the standard OI algortihm, as described in section 3.3.2, leading to the electromagnetic energy cluster, is used to separate the photon candidates from electrons. A maximum of one track is allowed, but only if it is of limited $p_{T}$ : $p_{T}<1+0.005 \cdot E_{T}$

- Iso4

The calorimeter isolation Iso4, is defined in the same way as for electron candidates, as detailed in section 4.2.3. A correction, due to additional energy from multiple interactions [74, 96], is subtracted from the isolation energy and shown in Equation 6.1. For photons with $E_{T}<20\left(E_{T}>20\right)$, it is required that Iso4 $/ E_{T}<0.1$ (Iso4 $<$ 
$\left.2.0+0.02 *\left(E_{T}-20.0\right)\right) \mathrm{GeV}$.

$$
V_{\text {Corr }}=0.28 \cdot\left(N_{\text {vertices }}-1\right)
$$

- TrackIso0.4

The calculation of the track isolation variable contains the sum of all tracks with a $z_{0}$ within $5 \mathrm{~cm}$ of the event vertex and in a cone of 0.4 around the photon candidate. Photon candidates must have a track isolation $<2+0.005 \cdot E_{T} \mathrm{GeV}$. This cut scales as a function of $E_{T}$ to ensure that the efficiency of the cut is flat as a function of the $E_{T}$.

\subsubsection{N-1 plots}

N-1 plots, for the photon selection cuts detailed in table 6.1 are made for the $e^{+} e^{-} \gamma$ and $\mu^{+} \mu^{-} \gamma$ candidates. N-1 photon requirements are applied to the $Z^{0}$ samples discussed in section 4 . The distribution for the Nth variable is plotted in figures 6.1 and 6.2 .

In Figure 6.1, the CES $|x|$ and $|z|$ distributions are plotted. As expected, the $E_{\text {had }} / E_{\text {em }}$ distribution is peaked at zero, similar to that of the electron. The simulation gives a good description of the data.

\subsection{Photon Identification and Reconstruction Efficiencies}

For the electron and muon efficiency corrections, the efficiency for each cut could be calculated in data and $\mathrm{MC}$ using a clean $Z^{0}$ sample. One tight electron(muon) was selected, and the second electron(muon) was used to 


\begin{tabular}{|l|l|c|}
\hline \hline Variable & Cut Value & Type of Cut \\
\hline \hline CES $|x|$ & $<21.0 \mathrm{~cm}$ & geometry \\
CES $|z|$ & $9.0<|z|<230.0 \mathrm{~cm}$ & geometry \\
$|\eta|$ & $<1.1$ & geometry \\
\hline$E_{\text {had }} / E_{\text {em }}$ & $<0.125||<0.055+0.00045 \times E$ & quality/isolation \\
Iso4 & $E_{T}>20:$ & \\
& $I s o 4<2.0+0.02 *\left(E_{T}-20.0\right) \mathrm{GeV}$ & isolation \\
& $E_{T}<20: I s o 4 / E_{T}<0.1 \mathrm{GeV}$ & \\
Number of tracks & $N_{\text {track }} \leq 1$ and $p_{T}<1+0.005 \cdot E_{T}$ & isolation \\
TrackIso0.4 & $<2+0.005 \cdot E_{T} \mathrm{GeV}$ & isolation \\
& $\left(\right.$ using tracks with $\left.\left|z_{0}-z_{\text {lep }}\right|<5 \mathrm{~cm}\right)$ & \\
\hline CES $\left(\chi_{\text {wire }}^{2}+\chi_{\text {strip }}^{2}\right) / 2$ & $<20$ & shape \\
2nd CES $<$ sliding & $<0.14 \cdot E_{T}\left(E_{T}<18\right)$ & shape \\
\hline \hline
\end{tabular}

Table 6.1: The list of photon selection cuts, with a brief note to categorise the cut to whether it is a geometry, isolation or shower shape requirement. 
determine the efficiency. For photons, no clean sample exists which can be used. A variety of different samples are therefore used to measure the efficiency corrections, $\frac{\epsilon_{D A T A}}{\epsilon_{M C}}$, for different aspects of our selection.

Excluding the photon ID cuts associated with geometry, the photon selection criteria can be categorised as either an isolation variable or as a variable
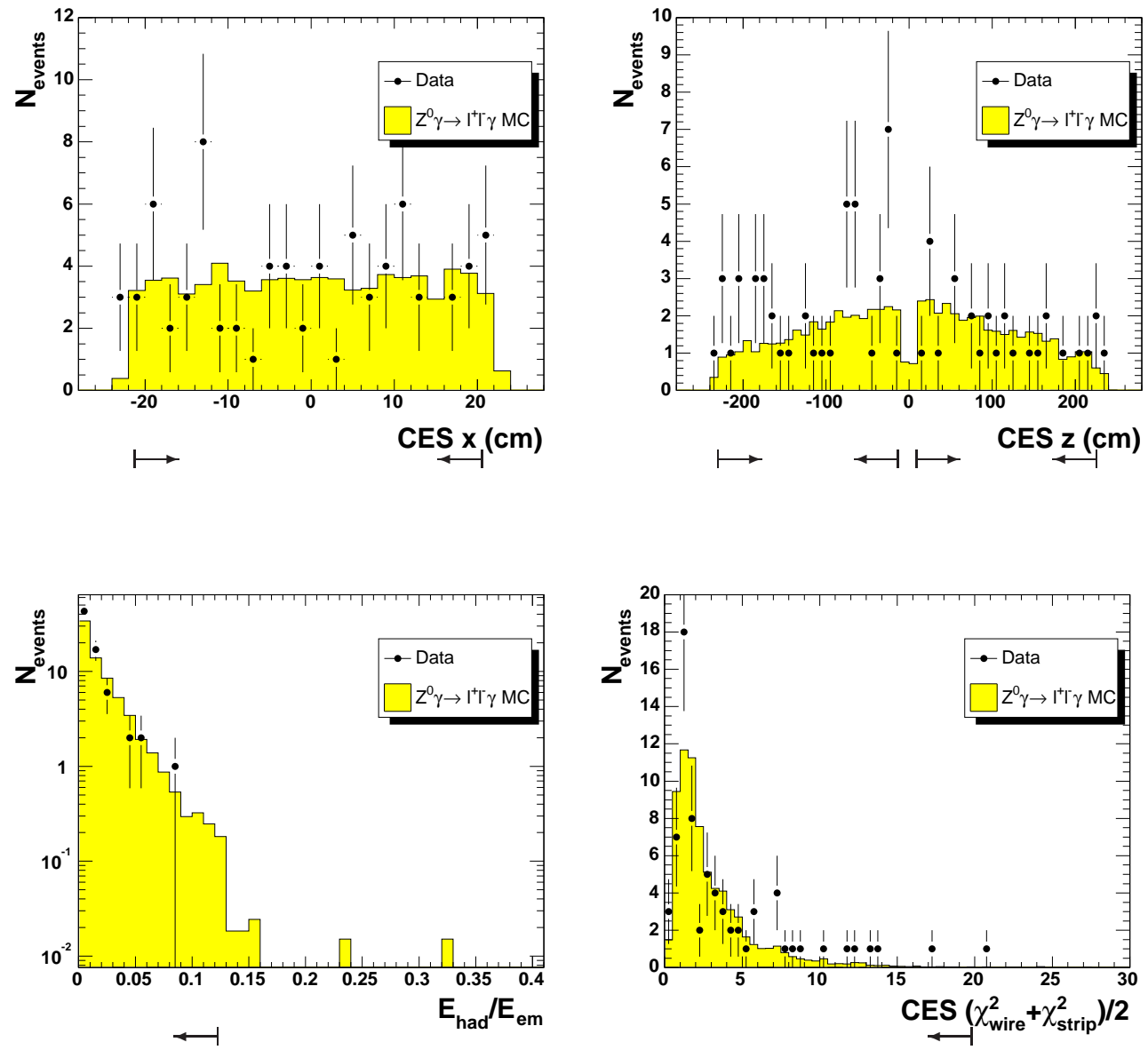

Figure 6.1: N-1 distributions for photon identification variables used for the selection of the events, plotted for data (dots) and MC (solid histogram). The arrow shows graphically the selection cut, where appropriate. The MC simulation is scaled to the luminosity of the data. 

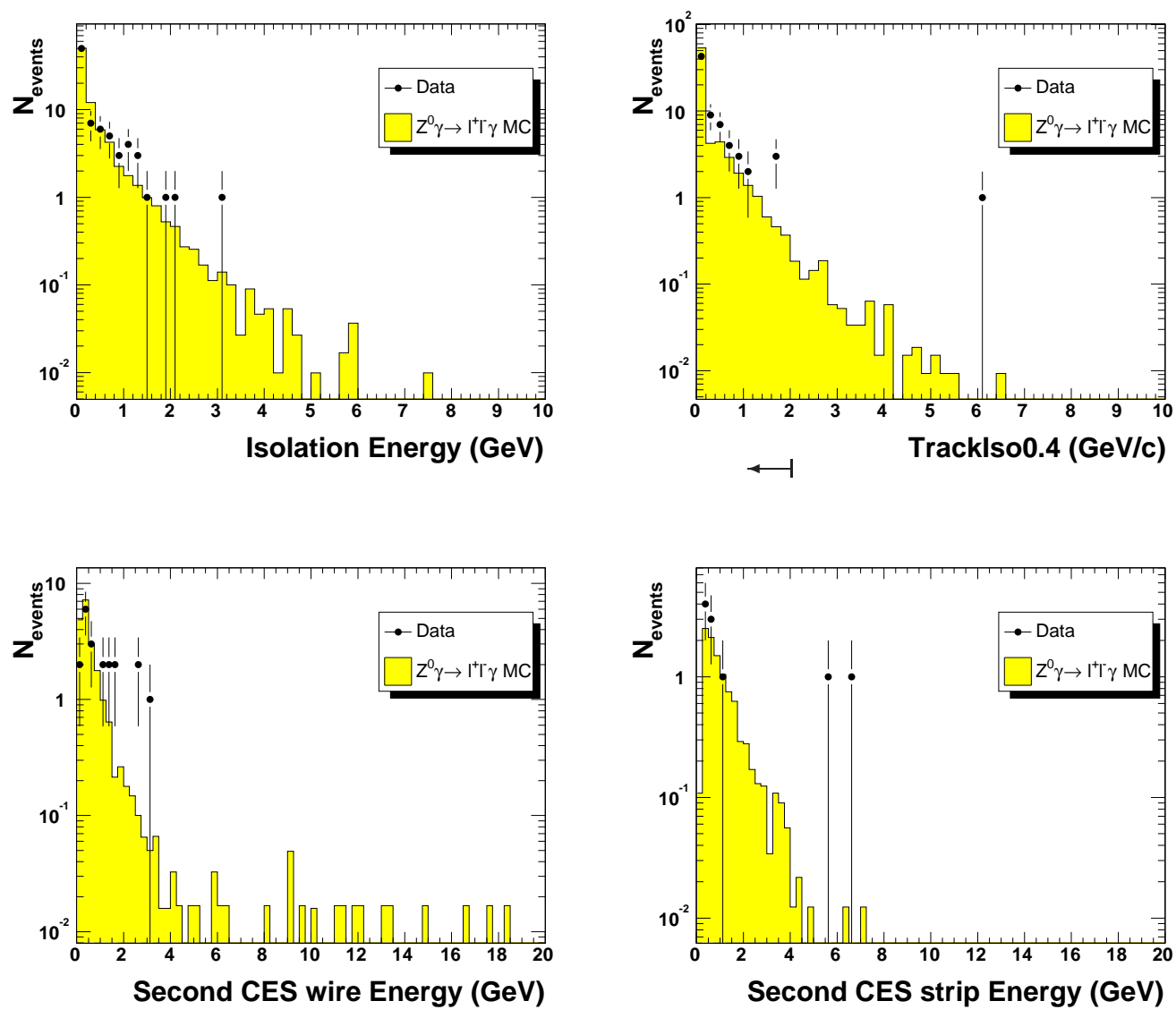

Figure 6.2: N-1 distributions for photon identification variables used for the selection of the events, plotted for $e^{+} e^{-} \gamma$ and $\mu^{+} \mu^{-} \gamma$ data (dots) and MC (solid histogram). The arrow shows graphically the selection cut, where appropriate. The MC simulation is scaled to the luminosity of the data.

associated with the shape of the electromagnetic shower (see table 6.1).

The efficiencies associated with the "isolation" and "shape" cuts have been measured elsewhere. A brief description of the techniques used and the results obtained are given in sections 6.2 .1 and 6.2 .2 respectively. The inefficiencies due to the rate of photon conversions is discussed separately in section 6.2.3. A summary of the efficiencies used for the photon selection for 
the $Z^{0} \gamma$ analysis is given in section 6.2.4.

\subsubsection{Efficiency of Isolation variables}

The underlying event resulting from the spectator parton interactions, or additional jets in the event, can deposit energy around otherwise isolated photons. These energy deposits can produce inefficiencies in the photon isolation selection cuts. In order to quantify this effect, the effect of the underlying event in the $W^{ \pm} \rightarrow e^{ \pm} \nu$ channel on the isolation variables is investigated. With the exception of the single electron, these events should mirror the underlying event in the $Z^{0} \gamma$ events. To avoid overlap with the electron cluster, a virtual cone is defined in the detector with the value of $\phi$ of the cone set to be $\phi_{e}+90^{\circ}$, where $\phi_{e}$ is the $\phi$ of the electron. A random $\eta$, with $|\eta|<1.1$, is chosen for the cone.

This method is explained in detail elsewhere [74]. The sample used is obtained by using the high $P_{T}$ electron triggered data sample described in section 4.2 .1 and selecting $W^{ \pm} \rightarrow e^{ \pm} \nu$ candidates with the cuts described in table 6.2.

\begin{tabular}{|c|}
\hline$W^{ \pm} \rightarrow e^{ \pm} \nu$ Cuts \\
\hline Tight Central Electron \\
$\mathbb{E}_{T}>25 \mathrm{GeV}$ \\
$30<M_{T}\left(e, \notin_{T}\right)<120 \mathrm{GeV} / \mathrm{c}^{2}$ \\
\hline
\end{tabular}

Table 6.2: $W^{ \pm}$selection cuts for random cone studies. $M_{T}(e, \nu)$ is the transverse mass calculated from the vectors associated with the electron and missing transverse energy, $\mathbb{E}_{T}: M_{T}\left(e, \mathbb{E}_{T}\right)=\sqrt{2 p_{T}^{e} \mathbb{E}_{T}\left(1-\cos \phi_{e}, \mathbb{E}_{T}\right)}$. 


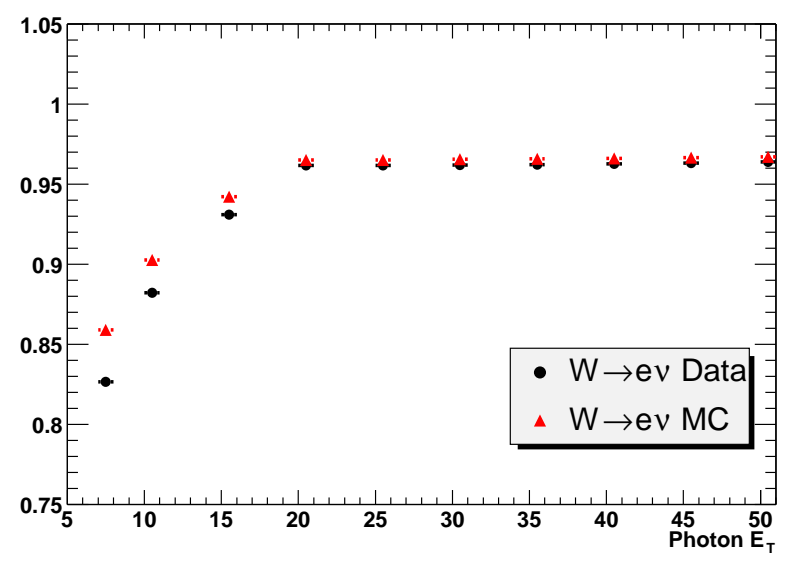

Figure 6.3: The isolation efficiency is plotted versus the assumed photon energy in $W^{ \pm} \rightarrow e^{ \pm} \nu$ data and Monte Carlo events. The data is for any number of vertices, while the Monte Carlo has only one vertex.

After a random cone of size $\Delta R=\sqrt{\Delta \phi^{2}+\Delta \eta^{2}}=0.4$ is constructed, isolation cuts are applied to it. The photon isolation cuts depend on $E_{T}$. Arbitary values of $E_{T}$ between 7 and $50 \mathrm{GeV}$ were chosen to be associated with the random cones. The cuts are applied sequentially and the efficiency defined as the simple ratio of the number of cones that pass the isolation cut to the total number of cones thrown. As the fiducial cuts are solely geometric, it is assumed that they have an efficiency of $100 \%$.

The calorimeter isolation, number of tracks, tracking isolation and second CES cluster cuts are made sequentially, with the individual studies made in turn. The most significant result is that for the calorimeter isolation.

The efficiency of the $I s o 4$ cut, in the $W^{ \pm}$sample for data and Monte Carlo, is plotted in Figure 6.3. The efficiency for both samples rises with energy until $20 \mathrm{GeV}$, becoming flat with respect to $E_{T}^{\gamma}$ for $E_{T}^{\gamma}>20 \mathrm{GeV}$. The change in slope at $20 \mathrm{GeV}$ is expected, as it is at this energy that the isolation 
cut changes from Iso4 $/ E_{T}<0.1 \mathrm{GeV}$ to $I s o 4<2.0+0.02 *\left(E_{T}-20.0\right)$ $\mathrm{GeV}$. For energies below $20 \mathrm{GeV}$, the efficiency of this cut is greater in MC than in data. The tighter cut is present at low $E_{T}$ to improve the background rejection.

The ratio of the two efficiency curves in Fig 6.3 was taken and fitted with a third order polynomial to give the correction to the $\mathrm{MC}$ efficiency as a function of photon $E_{T}$. The fitted function given in Eq 6.2 [74].

$$
\begin{aligned}
E_{T}<20 \mathrm{GeV}: & \\
& E f f_{\text {corr }}=\operatorname{Eff} f_{M C}\left(0.89+0.0175 E_{T}^{\gamma}-0.00104 E_{T}^{\gamma 2}+2 \times 10^{-5} E_{T}^{\gamma 3}\right) \\
E_{T}>20 \mathrm{GeV}: & \\
& E f f_{\text {corr }}=0.98 .
\end{aligned}
$$

Table 6.3 shows the correction factors derived using the random cone technique for all the isolation variables.

\begin{tabular}{|l|c|c|}
\hline Cut & Correction Factor & Error \\
\hline Cal Iso & $\mathrm{f}\left(E_{T}\right)$ & $\pm 1.0 \%$ \\
N3D & 1.0 & $\pm 0.3 \%$ \\
Track Iso & 1.0 & $\pm 0.3 \%$ \\
Ces $\chi^{2}$ & 1.0 & $\pm 1.0 \%$ \\
2nd CES E & 0.99 & $\pm 1.0 \%$ \\
\hline
\end{tabular}

Table 6.3: Correction factors used to scale the MC in data [74]. The calorimeter isolation depends on $E_{T}^{\gamma}$. $f\left(E_{T}\right)=0.89+0.0175 \times E_{T}-0.00104 \times E_{T}^{2}+$ $2 \times 10^{-5} \times E_{T}^{3}$ for $E_{T}<20 \mathrm{GeV}$, and $f\left(E_{T}\right)=0.98$ for $E_{T}>20 \mathrm{GeV}$. 


\subsubsection{Efficiency of Shower Shape Identification Criteria}

The behaviour of electrons and photons in the calorimeters are almost identical. They produce showers in exactly the same way, the only difference being that photon showers develop slightly later and that electrons will emit bremsstrahlung radiation. A clean "photon" sample can be obtained by subtracting the track of electrons in clean $Z^{0} \rightarrow e^{+} e^{-}$and conversion samples, and requiring $E / P<1.1$ to remove bremsstrahlung effects. This means that the efficiency of the calorimeter based photon identification cuts can be measured using electrons. The $Z^{0} \rightarrow e^{+} e^{-}$and conversion $\left(\gamma \rightarrow e^{+} e^{-}\right)$samples provide electrons with an $E_{T}$ range of $25<E_{T}<45$ and $7<E_{T}<20$ GeV respectively. The efficiencies of the calorimeter based identification have been derived elsewhere[75] using these samples. The results are summarised in table 6.4 .

\begin{tabular}{|l|c|c|c|c|}
\hline Cut & Random Cone & $Z \rightarrow e e$ & Conversion Sample & Value Used \\
\hline Iso & $\mathrm{f}\left(E_{T}\right) \pm 0.01$ & - & $1.01 \pm 0.005$ & $\mathrm{f}\left(E_{T}\right) \pm 0.01$ \\
$\mathrm{~N} 3 \mathrm{D}$ & $1.0 \pm 0.003$ & - & $0.977 \pm 0.006$ & $0.99 \pm 0.01$ \\
Trk Iso & $1.0 \pm 0.003$ & - & $0.988 \pm 0.005$ & $0.99 \pm 0.005$ \\
CES $\chi^{2}$ & - & $1.01 \pm 0.01$ & $0.98 \pm 0.01$ & $0.99 \pm 0.015$ \\
CES strip E & $0.995 \pm 0.01$ & $1.01 \pm 0.02$ & $1.00 \pm 0.003$ & $1.00 \pm 0.01$ \\
CES wire E & $0.995 \pm 0.01$ & $0.99 \pm 0.01$ & $0.987 \pm 0.004$ & $0.99 \pm 0.005$ \\
\hline
\end{tabular}

Table 6.4: The summary of the correction factor to scale the $Z \gamma$ MC. $f\left(E_{T}\right)=$ $0.89+0.0175 \times E_{T}-0.00104 \times E_{T}^{2}+2 \times 10^{-5} \times E_{T}^{3}$ for $E_{T}<20 \mathrm{GeV}$, and $f\left(E_{T}\right)=0.98$ for $E_{T}>20 \mathrm{GeV}$. 
Each correction factor in table 6.4, has been measured using at least two different methods. The value applied in this analysis is the average of the two methods. The systematic error is taken to be the difference of the two measurements.

\subsubsection{Rate of Conversions}

A fraction of prompt photons will convert in the tracking systems to an electron-positron pair. These conversions will be rejected by the N3D track cut producing an inherent efficiency loss due to conversions. This efficiency loss was measured to be $10.21 \pm 0.25 \%$ in simulation. However, this result is highly dependent on the amount of material in the simulation being correct. The more material a photon has to pass through, the greater the chance of the photon converting. The amount of material in the simulation is underestimated by about $30 \%$ in the MC samples used for this analysis. The amount of material simulated for the ZGAMMA MC, in the inner tracking volume at $R<42 \mathrm{~cm}$, is $15 \% X_{0}$. Studies indicate that another $(4.5 \pm 1.5) \% X_{0}$ are present in the data [115]. Therefore the conversion probability in the data is estimated to be $10.21 \times \frac{4.5+15}{15}=13.3 \pm 1.3 \%$. A correction factor is therefore applied to the simulation of $(100-13.3) /(100-10.2)=0.97$, with a systematic error of $1.5 \%$ (rounded up from $1.3 \%$ ).

\subsubsection{Efficiency Summary}

In summary, the efficiency correction associated with the photon selection consists of an $E_{T}^{\gamma}$ dependent correction factor applied to the $Z \gamma$ MC sample 
of

$$
\begin{aligned}
E_{T}<20 \mathrm{GeV}: & \\
& \epsilon_{\text {Data }} / \epsilon_{M C}=0.89+0.0175 \times E_{T}-0.00104 \times E_{T}^{2}+2 \times 10^{-5} \times E_{T}^{3} \\
E_{T} \geq & 20 \mathrm{GeV}: \\
& \epsilon_{\text {Data }} / \epsilon_{M C}=0.984 .
\end{aligned}
$$

plus an overall correction factor of $0.960 \pm 0.023 \%$. The sources of these corrections are summarised in table 6.4. In addition, the correction factor for the conversion efficiency is $0.970 \pm 0.015 \%$.

\section{3 $Z^{0} \gamma$ Acceptance and Identification Effi- ciencies}

The product of the acceptance and efficiency for the $Z^{0} \gamma$ cross-section measurement is calculated using the same method as described in section 5.2. The acceptance $\times$ efficiency is calculated from the ZGAMMA MC sample (see section 2.2), as the fraction of generated events (see section 2.2), that pass the event selection criteria. 


\begin{tabular}{|l|c|c|c|}
\hline & \multicolumn{2}{|c|}{$e^{+} e^{-} \gamma$} & $\mu^{+} \mu^{-} \gamma$ \\
& C-C & C-P & CMUP/X+trk \\
\hline 1 Tight Lepton & \multicolumn{2}{|c|}{$30.75 \%$} & $27.35 \%$ \\
1 Tight and 1 Loose lepton & $5.66 \%$ & $6.74 \%$ & $8.75 \%$ \\
Opposite Charge & $5.54 \%$ & - & $8.75 \%$ \\
$40<m^{r e c}(l l)<130 \mathrm{GeV} / \mathrm{c}^{2}$ & $5.53 \%$ & $6.74 \%$ & $8.71 \%$ \\
Photon Cuts & $1.03 \%$ & $0.80 \%$ & $1.97 \%$ \\
\hline
\end{tabular}

Table 6.5: Acceptance $\times$ Efficiency for $Z^{0} \gamma \rightarrow e^{+} e^{-} \gamma$ and $Z^{0} \gamma \rightarrow \mu^{+} \mu^{-} \gamma$ 


\section{Chapter 7}

\section{Estimation of Background Contributions}

The only significant source of background to $Z^{0} \gamma$ production, is $Z^{0}+$ jet, where the jet fragments in such a way as to pass the photon selection criteria and thus be misidentified. In order to estimate this contribution, the rate at which a jet originating from a quark or a gluon fakes an isolated photon in the central calorimeter is determined. Any photon that is due the decay of a meson (e.g. $\pi_{0} \rightarrow \gamma \gamma, \eta \rightarrow \gamma \gamma$ or $\left.K_{s}^{0} \rightarrow \pi_{0} \pi_{0} \rightarrow \gamma \gamma+X\right)$ is classified as "fake" and any photon that is created in the hard scattering process or radiated off a quark is classified as a "prompt" photon. The event samples used in this chapter come from the JET_20, JET_50, JET_70 and JET_100 triggered samples[51]. These samples demand at least one jet to have an $E_{T}$ greater than 20, 50, 70 and $100 \mathrm{GeV}$ respectively.

An overview of the fake rate method and its application is given in section 7.1. A description of the jet triggered dataset used to measure the rate and the measurement of the fake rate itself is given in sections 7.2 and 7.3 
respectively. To account for prompt photons in the jet datasets used, a correction is applied to the fake rate. This correction is derived in section 7.4, with the resulting fake rate given in section 7.5. The application of the fake rate is described in section 7.6 and the systematic errors are given in section 7.7. A cross-check of the method is shown in section 7.8 and a discussion of other sources of background is presented in section 7.10.

\subsection{Fake Rate Definition}

The aim is to measure a fake rate (i.e., the rate at which a jet passes the photon selection) in a jet triggered sample and then apply it to the $Z^{0}+$ jet sample. The number of background events from jets faking photons in the $Z^{0} \gamma$ analysis is given by

$$
\begin{aligned}
N_{B G}\left(E_{T}^{\gamma}\right) & =\int_{0}^{N_{\text {jet }}(Z)} P_{\text {jet } \rightarrow \gamma}^{Z}\left(E_{T}^{\text {jet }}\right) \times \mathrm{d} N_{Z} / \mathrm{d} E_{T}^{\text {jet }} \times z\left(E_{T}^{\text {jet }}, E_{T}^{\gamma}\right) \mathrm{d} E_{T}^{\text {jet }} \\
& =\int_{0}^{N_{\text {jet }}(Z)} P_{\text {jet } \rightarrow \gamma}^{j}\left(E_{T}^{\text {jet }}\right) \times \frac{\mathrm{d} N_{Z} / \mathrm{d} E_{T}^{\text {jet }}}{\mathrm{d} N_{\text {jet }} / \mathrm{d} E_{T}^{\text {jet }}} \mathrm{d} N_{j e t} / \mathrm{d} E_{T}^{\text {jet }} \times z_{j}\left(E_{T}^{\text {jet }}, E_{T}^{\gamma}\right) \mathrm{d} E_{T}^{\text {jet }}(7.2)
\end{aligned}
$$

where

- $P_{\text {jet } \rightarrow \gamma}^{Z}\left(E_{T}^{\text {jet }}\right)$ is the $E_{T}^{\text {jet }}$ dependent probability of a jet faking a photon for jets in the $Z^{0}$ sample.

- $\mathrm{d} N_{\text {jet }} / d E_{T}^{\mathrm{jet}}$ is the $E_{T}^{\mathrm{jet}}$ distribution in a jet triggered sample.

- $\mathrm{d} N_{Z} / d E_{T}^{\mathrm{jet}}$ is the $E_{T}^{\mathrm{jet}}$ distribution in $Z^{0}$ events.

- $P_{\text {jet } \rightarrow \gamma}^{j}\left(E_{T}^{\text {jet }}\right)$ is the $E_{T}^{\text {jet }}$ dependent probability of a jet faking a photon for jets in the jet sample.

- The term $\frac{\mathrm{d} N_{Z} / \mathrm{d} E_{T}^{\text {jet }}}{\mathrm{d} N_{\text {jet }} / \mathrm{d} E_{T}^{\text {jet }}}$ cancels if the $E_{T}^{\text {jet }}$ distributions are the same in the jet sample used to measure the fake rate and fragmentation, and the 
$Z^{0}$ sample that it is applied to. Figure 7.1 shows the $E_{T}^{\text {jet }}$ distribution for $Z^{0}$ data and for the 2 nd and $3 \mathrm{rd}, 4$ th, 5 th etc. highest $E_{T}$ jet in the jet triggered samples. The sample of jets consisting of the 3rd, 4 th, 5th etc. highest $E_{T}$ jets will be referred to as the "345th" jet sample.
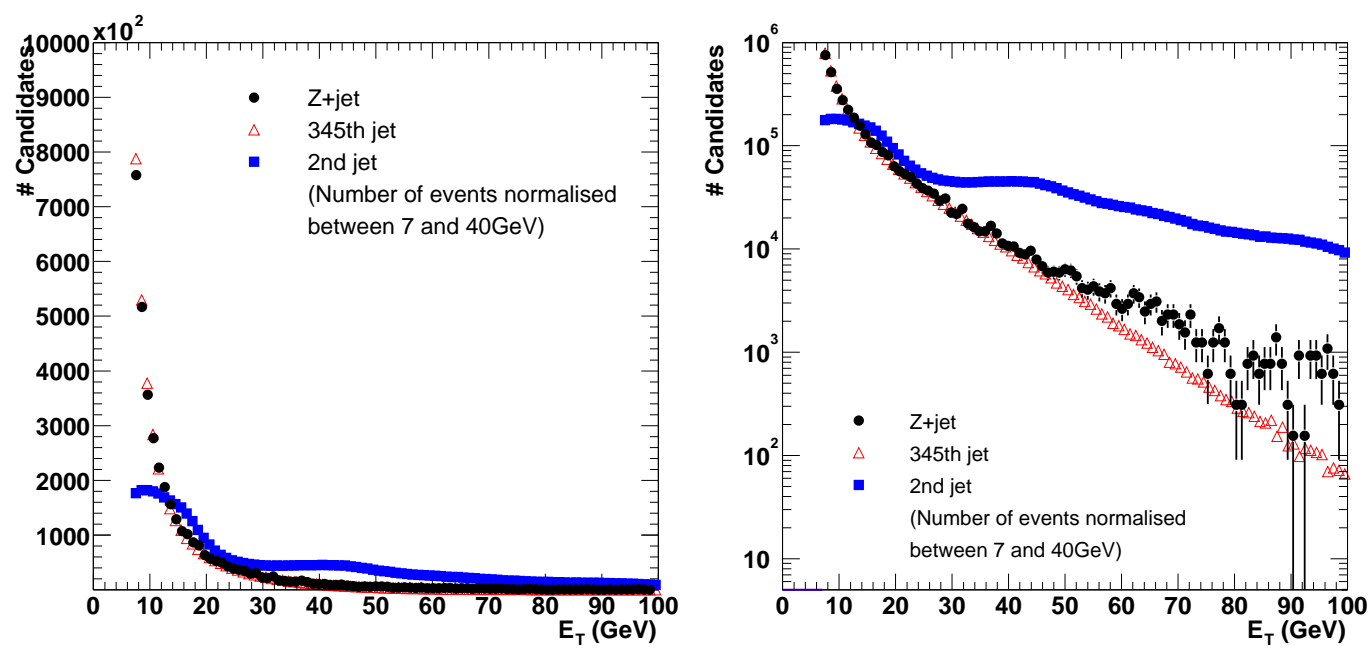

Figure 7.1: The $E_{T}^{\text {jet }}$ distribution $\mathrm{d} N / \mathrm{d} E_{T}^{\text {jet }}$ for jets in the $Z^{0}$ sample (black points), the 2 nd jet in the jet samples (blue squares) and the 345th jet in the jet samples (red open triangles).

For the 345 th jet in the jet samples the $E_{T}^{\text {jet }}$ distribution is very similar to that in the $Z^{0}$ sample. The 345 th jet sample is therefore chosen to be used to measure $P_{\text {jet } \rightarrow \gamma}^{j}\left(E_{T}^{\text {jet }}\right)$. The 2 nd jet sample is used for cross-check purposes only, as discussed in section 7.7.2.

- $z\left(E_{T}^{\mathrm{jet}}, E_{T}^{\gamma}\right)$ is a matrix which gives the probability of a jet of $E_{T}^{\mathrm{jet}}$ to be measured as $E_{T}^{\gamma}$. This matrix is measured in inclusive jet events and depends on the $E_{T}^{\text {jet }}$ distribution since smearing effects due to limited resolution can change this matrix. To minimise such effects a sample 
which has a very similar $E_{T}^{\text {jet }}$ distribution as the $Z^{0}+$ jet sample is required. Systematics derived to fully account for the fragmentation and the application of this factor are discussed in sections 7.7.3 and 7.7.4.

The contributions are summed over all $Z+$ jet candidates in the exact same data set used to select the $Z^{0} \gamma$ candidates, detailed in section 4 to obtain the final background estimate.

\subsection{Data Sample}

The data used for the fake rate measurement are the so-called JET_20, JET_50, JET_70 and JET_100 triggered samples. The trigger object is a jet of cone size $\Delta R=\sqrt{\Delta \eta^{2}+\Delta \phi^{2}}=0.7$. The sum of the $E_{T}$ of the towers in the jet must be greater than 20(50/70/100) GeV to pass the JET_20(50/70/100) trigger [51]. The same good run requirements as used for the $Z_{C C}^{0}$ sample (see section 4.2.2) are required.

From the events in this sample of $202 \mathrm{pb}^{-1}$, jets of cone size $\Delta R=0.4$ with $E_{T}>7 \mathrm{GeV}$ are selected. Throughout this chapter, the jets are numbered according to their $E_{T}$. The "1st" jet refers to the highest $E_{T}$ Jet, the "2nd" jet refers to the 2 nd highest $E_{T}$ jet and so on. The following requirements are made upon the jet sample:

- The highest $E_{T}$ jet, the "trigger jet", is excluded from this analysis in order to remove any trigger bias.

- Only events where the 1 st and 2 nd jet are separated by $\Delta R<0.8$ are used. 
- Additional jets (3rd, 4 th, 5 th.. ) are required to be separated by $\Delta R>$ 0.8 from all other ("previous") jets. The motivation for this cut comes from figure 7.2. In the software, any photon will also be classified as a "jet". The peak at values close to zero shows the photon objects which have a good matching to an associated jet, ie. they are the same object. The broad distribution comes from other jets in the event. The choice of $\Delta R>0.8$ ensures that the photons are well separated from the jet.

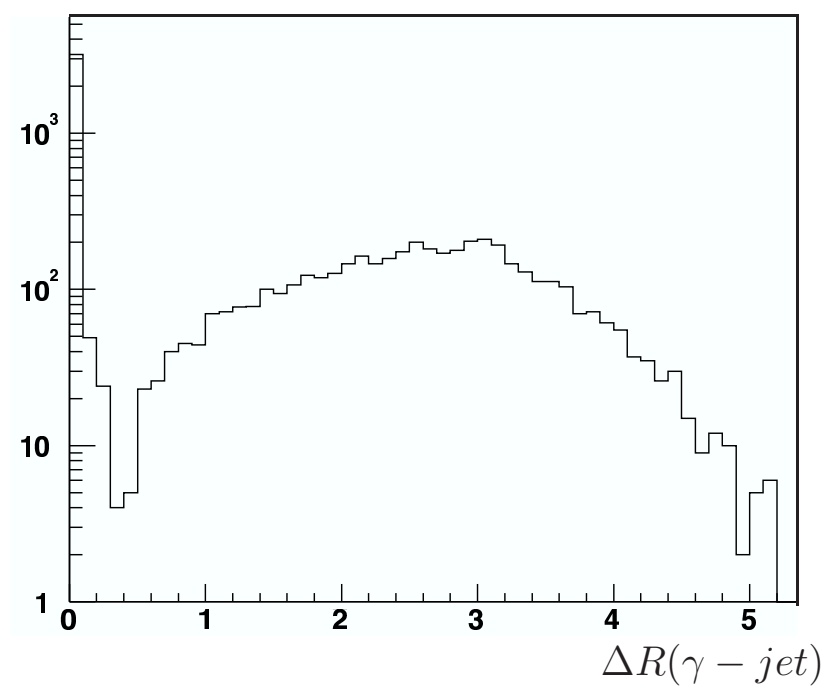

Figure 7.2: The $\Delta R$ distribution between the prompt $\gamma$ candidate and any "jet' in ZGAMMA MC.

\subsection{The Fake Probability}

The measurement of $P_{\text {jet } \rightarrow \gamma}\left(E_{T}^{\text {jet }}\right)=\frac{N_{\text {jet } \rightarrow \gamma}}{N_{\text {jet }}}$ requires a sample which contains only jets and no prompt photons. Since no such dataset exists, the prompt photon contamination $F_{\gamma}$ is estimated in the samples used: 


$$
F_{\gamma}=\frac{N_{\gamma}}{N_{\gamma}+N_{\text {jet } \rightarrow \gamma}}=\frac{N_{\gamma}}{N_{\gamma-\text { candidate }}}
$$

The fraction of fake photons in the photon candidates sample are defined as:

$$
F_{\text {jet }}=\frac{N_{\text {jet } \rightarrow \gamma}}{N_{\gamma-\text { candidate }}}=1-F_{\gamma}
$$

Then:

$$
\begin{aligned}
P_{\text {jet } \rightarrow \gamma}\left(E_{T}^{\text {jet }}\right) & =\frac{N_{\gamma-\text { candidate }}}{N_{\text {jet }}} \times \frac{N_{\text {jet } \rightarrow \gamma}}{N_{\gamma-\text { candidate }}}=\frac{N_{\gamma \text {-candidate }}}{N_{\text {jet }}} \times F_{\text {jet }} \\
& \equiv P_{\text {raw }}\left(E_{T}^{\text {jet }}\right) \times F_{\text {jet }} .
\end{aligned}
$$

The measurement of $P_{\text {raw }}\left(E_{T}^{\text {jet }}\right)$ is shown in figure 7.3 to be consistent between the $J E T \_20$ and the sum of the $J E T \_50, J E T \_70$ and $J E T \_100$ samples. It is about $0.4 \%$ at the lowest $E_{T}^{\text {jet }}$ values of $7 \mathrm{GeV}$ and falls then exponentially with $E_{T}^{\text {jet }}$ to about $0.2 \%$ at $E_{T}^{\text {jet }}=50 \mathrm{GeV}$. The different data samples all agree to within less than $5 \%$ of each other. In the following sections, $P_{\text {jet } \rightarrow \gamma}\left(E_{T}^{\text {jet }}\right)$ will be referred to as the "true fake rate" and $P_{\text {raw }}\left(E_{T}^{\text {jet }}\right)$ as the "raw fake rate".

\subsection{Contamination of the sample with True Photons}

There are several variables which can be used to measure $F_{j e t}$ :

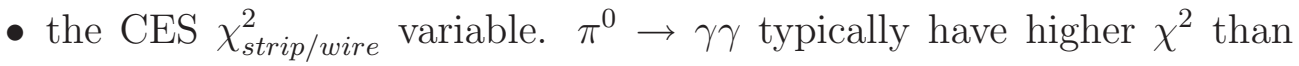
prompt photons (where $\chi^{2}=\left(\chi_{\text {strip }}^{2}+\chi_{\text {wire }}^{2}\right) / 2$. 


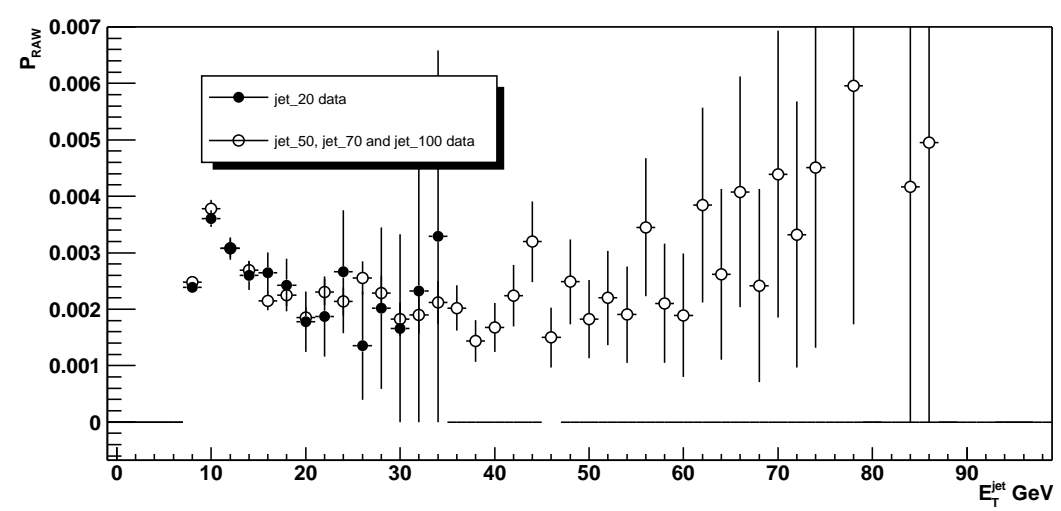

Figure 7.3: $P_{\text {raw }}$ versus jet $E_{T}$ comparing $J E T \_20$ (open points) and $J E T \_50$, JET_70 and JET_100 data (full points).

- the transverse energy in a cone of radius 0.4 around the photon candidate Iso4: the background is usually produced as part of a jet and thus the transverse energy around the photon candidate is typically higher for the background than for the signal.

- the CPR hit rate. The CPR is located between the solenoid and the calorimeter. Photons converting in the coil are recorded as charge, $Q$. The conversion probability per photon is about $P_{\gamma}=60 \%$. The probability for at least one photon, from $\pi^{0} \rightarrow \gamma \gamma$, converting is about $P_{\pi^{0}}=1-\left(1-P_{\gamma}\right)^{2}=84 \%$. By measuring the fraction of photon candidates which deposit a significant charge in the CPR, the fraction of prompt photons can thus be calculated.

This analysis uses three independent methods, based on these variables to determine the prompt photon fraction. These are explained in detail in 7.4.2, 7.4.1 and 7.4.3 and combined in section 7.4.4. 


\subsubsection{CES method}

This method developed elsewhere, is described in detail in [76] and uses the CES detector as described in section 3.3.4. The Run 2 implementation is described in [77].

A single prompt photon can be differentiated from background by the shape of the electromagnetic shower profile in the calorimeter. A single isolated photon shower will naturally be narrower than multiple showers with some spatial separation, for example showers caused by the decay of a $\pi^{0}$ to two photons. The philosophy of this statistically based method is to attach a weight to an event according to how signal-like or background-like it is. With a large statistical sample containing both single showers and $\pi^{0}$ induced showers, it is possible to evaluate the fraction of $\pi^{0}$ events by observing the ratio of "narrow" to "wide" showers, i.e., the ratio of showers that are consistent with a single electromagnetic shower to those that are "too wide" to be consistent.

A statistical based method is required since particle-by-particle ID is impossible. The typical opening angle for photons from the decay of a $5 \mathrm{GeV}$ $\pi^{0}$ is $3^{\circ}$, dropping to $0.3^{\circ}$ for a $50 \mathrm{GeV} \pi^{0}$. At the radius of the CES chamber this corresponds to separations of $10 \mathrm{~cm}$ and $1 \mathrm{~cm}$ respectively. The minimal separation of the photons is approximately given by $\frac{50 \mathrm{cmGeV} / \mathrm{c}}{P_{T}}$. Typical shower sizes in the electromagnetic calorimeter are of the order of its Molíere radius[116] of $3.5 \mathrm{~cm}$. This means that for $P_{T}$ values above $15 \mathrm{GeV} / \mathrm{c}$, it is not possible to resolve individual showers from two photons from a neutral meson decay. This makes particle-by-particle identification impossible.

A variable $\tilde{\chi}^{2}$ is defined which describes how well a shower compares to a 
single shower from electron test beam data. The chamber energies are clustered in both the strip and wire views. Any wire (or strip) with at least 0.5 $\mathrm{GeV}$ energy are used as seed candidates and ranked in descending order of energy. An eleven wire (or strip) "window" is placed around the highest energy "seed" wire (strip). The clustering begins with this seed wire(strip) and continues through all candidates with the elimination of wires(strips) used in previously found clusters. A shower fit is then performed over the eleven wires(strips) for each cluster. The energy sum of the eleven wires(strips) is scaled to 1.0, so the fit only involves the relative energy deposits in each wire(strip). Since a single prompt photon should shower in the same manner as a single electron of the same energy, the fitting procedure is optimised using test beam electrons with energies in the range $10 \mathrm{GeV}$ to $100 \mathrm{GeV}$. An approximate $\chi^{2}$ per degree of freedom, known as $\chi^{2}$ is used, that is independent of energy:

$$
\chi^{2}=\sum_{i=1}^{11}\left(p_{i}-y_{i}\right)^{2} / \tilde{\sigma}_{i}^{2}
$$

where $p_{i}$ are the relative strip(wire) energies, $y_{i}$ are the expected energy and

$$
\sigma_{i}^{2} \equiv 4\left[(0.026)^{2}+(0.096)^{2} y_{i}\right]\left(\frac{10 \mathrm{GeV}}{E}\right)^{0.747} .
$$

$E$ is the energy of the cluster.

The average $\chi^{2}$ variable is used:

$$
\chi^{2}=\frac{\chi_{\text {strip }}^{2}+\chi_{w i r e}^{2}}{2} .
$$

$\tilde{\chi}_{\text {strip }}^{2}$ and $\tilde{\chi}_{w i r e}^{2}$ are the contributions given by the strip and wire clusters respectively.

The signal and background efficiencies, $\epsilon_{\gamma}$ and $\epsilon_{b}$ are defined as the ratio of the number of events with $\tilde{\chi}^{2}<4$ to $\tilde{\chi}^{2}<20$. If $N_{\gamma}^{\tilde{\chi}^{2}<4}\left(N_{b}^{\tilde{\chi}^{2}<4}\right)$ is the 
number of signal (background) events with $\tilde{\chi}^{2}<4$ and $N_{\gamma}^{\tilde{\chi}^{2}<20}\left(N_{b}^{\tilde{\chi}^{2}<20}\right)$ is the number of signal (background) events with $\tilde{\chi}^{2}<20$ then:

$$
\epsilon_{\gamma}=\frac{N_{\gamma}^{\tilde{\chi}^{2}<4}}{N_{\gamma}^{\tilde{\chi}^{2}<20}}
$$

and

$$
\epsilon_{b}=\frac{N_{b}^{\tilde{\chi}^{2}<4}}{N_{b}^{\tilde{\chi}^{2}<20}} .
$$

The efficiencies $\epsilon_{\gamma}$ and $\epsilon_{b}$ are plotted in figure 7.4 as a function of $E_{T}$. By measuring this fraction in the data $\left(\epsilon_{\text {data }}\right)$, the background contribution can be calculated.

$$
N_{b}=\frac{\left(\epsilon_{d a t a}-\epsilon_{\gamma}\right) N_{t o t a l}}{\left(\epsilon_{b}-\epsilon_{\gamma}\right)}
$$

where:

$$
\begin{aligned}
N_{\gamma} & =N_{\text {total }}-N_{b} \\
\epsilon N_{\text {total }} & =\epsilon_{\gamma} N_{\gamma}+\epsilon_{b} N_{b}
\end{aligned}
$$

$F_{\text {jet }}$ is simply given by the ratio of $N_{b} / N_{\text {total }}$. This method ultimately breaks down at around $40 \mathrm{GeV}$, due to the two photon showers of $\pi^{0} \rightarrow \gamma \gamma$ becoming too similar to prompt $\gamma$ showers (i.e. $\epsilon_{\gamma} \approx \epsilon_{b}$ ).

\subsubsection{Iso vs. $\chi^{2}$ :}

For background such as $\pi^{0} \rightarrow \gamma \gamma$, there is no correlation between the transverse energy in a cone of radius $0.4, I s o 4$, and the CES $\chi^{2}$ variable. A $\pi^{0} \rightarrow \gamma \gamma$ can have a range of values of Iso4 depending on the fragmentation 
of the jet, and $\chi^{2}$, depending on its energy. The assumption that these quantities are uncorrelated has been verified in MC samples (see appendix A.2.1).

The 2-dimensional distribution of $I$ so 4 and $\chi^{2}$ in data can be used to give an estimate $F_{\text {jet }}$. Four regions are defined in the 2-dimensional plane of Iso vs. $\chi^{2}$. The regions are given in table 7.1 for $E_{T}^{\gamma}<20$ and $E_{T}^{\gamma}<20 \mathrm{GeV}$. The data are shown in figure 7.5. Region $\mathrm{C}$ corresponds to the signal region and region D is a pure background region. Assuming that there is no signal in $\mathrm{D}$ and that the two observables are uncorrelated the background in the signal region is predicted as:

$$
N_{B G}=\frac{N_{B} \cdot N_{A}}{N_{D}}
$$

and thus

$$
\begin{aligned}
F_{j e t} & =\frac{N_{B G}}{N_{C}} \\
& =\frac{N_{B} \cdot N_{A}}{N_{D} \cdot N_{C}}
\end{aligned}
$$

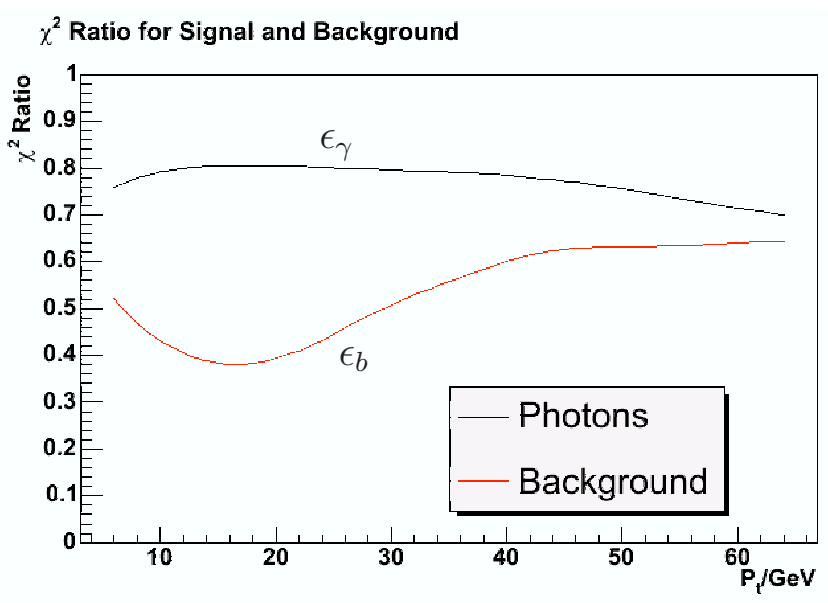

Figure 7.4: The signal $\left(\epsilon_{\gamma}\right)$ and background $\left(\epsilon_{b}\right)$ templates for the CES method 
is the fraction of background in the sample.

\begin{tabular}{|l||l|l|l|l|}
\hline Region & \multicolumn{2}{||}{$E_{T}^{\gamma}>20 \mathrm{GeV}$} & \multicolumn{2}{c|}{$E_{T}^{\gamma}<20 \mathrm{GeV}$} \\
\hline $\mathrm{A}$ & $\frac{(I s o 4-2)}{\left(20-E_{T}\right)}>0.06$ & $\chi^{2}<20$ & Iso4 $/ E_{T}>0.2$, & $\chi^{2}<20$ \\
$\mathrm{~B}$ & $\frac{(I s o 4-2)}{\left(20-E_{T}\right)}<0.02$, & $\chi^{2}>20$ & Iso4 $/ E_{T}<0.1$, & $\chi^{2}>20$ \\
$\mathrm{C}$ & $\frac{(I s o 4-2)}{\left(20-E_{T}\right)}<0.02$, & $\chi^{2}<20$ & Iso4 $/ E_{T}<0.1$, & $\chi^{2}<20$ \\
$\mathrm{D}$ & $\frac{(I s o 4-2)}{\left(20-E_{T}\right)}>0.06$, & $\chi^{2}>20$ & Iso4 $/ E_{T}>0.2$, & $\chi^{2}>20$ \\
\hline
\end{tabular}

Table 7.1: Cut values to define the regions used for the Iso vs. $\chi^{2}$ method for two regions in $E_{T}^{\gamma}$.
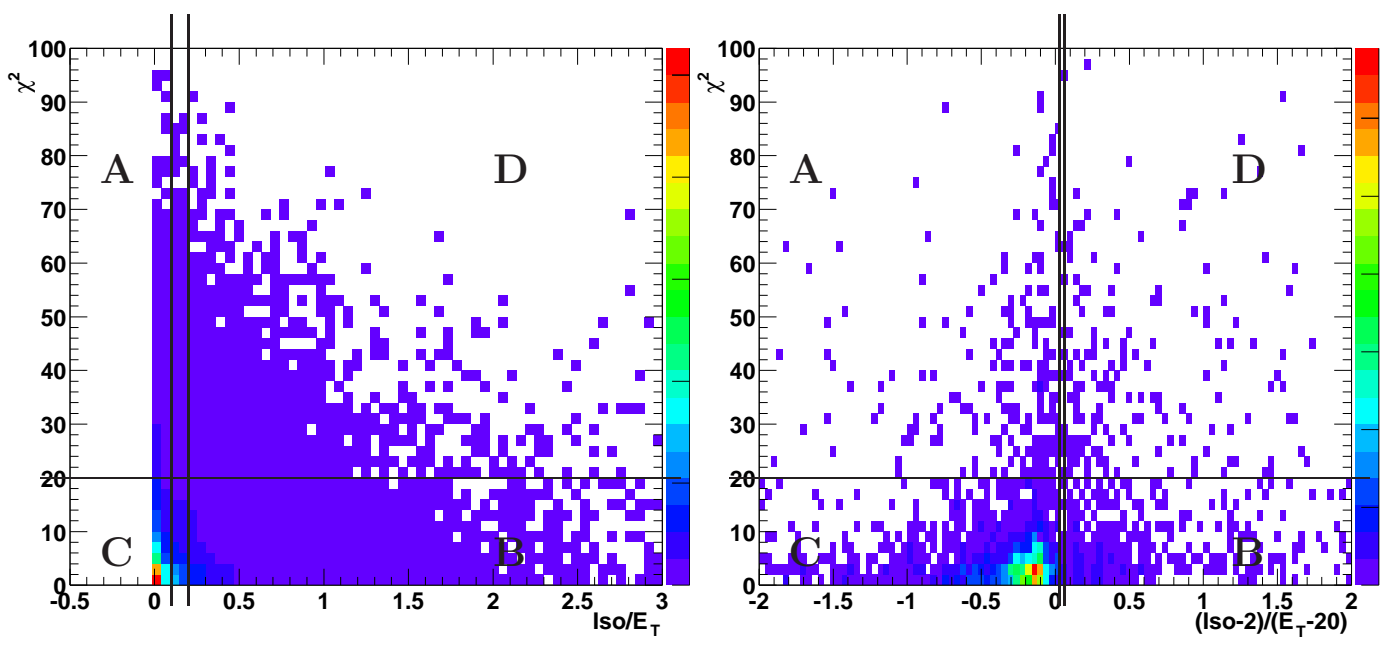

Figure 7.5: Iso4 $/ E_{T}$ (left) and (Iso4 -2$) /\left(20-E_{T}^{\gamma}\right)$ versus $\chi^{2}$. Illustrated are the four regions $\mathrm{A}, \mathrm{B}, \mathrm{C}$ and $\mathrm{D}$ used for determining the QCD background fraction $F_{\text {jet }}$ (see table 7.1 )

The effect of the choice of the boundaries for the four regions A, B, C and $\mathrm{D}$ was investigated and no systematic dependence is found. Details can be found in appendix A.2.2. 


\subsubsection{CPR method}

This method is described in detail in [76]. The Run 2 implementation is described in $[77,78]$. In addition to the photon selection cuts of table 6.1 , the following cuts are applied:

- No track is present that extrapolates to the signal tower $\mathrm{N} 3 \mathrm{D}=0$.

- no track within the half wedge of the candidate photon should be within the $\Delta x$ of $\pm 10 \mathrm{~cm}$ of the CPR position as extrapolated from the measured CES cluster. This is done because of the segmentation of the CPR into 4 half wedges. If any charged particle goes into the same half wedge, the CPR hit method is not applicable.

- $14<z_{C E S}<217 \mathrm{~cm}$ and $\left|x_{C E S}\right|<17.5 \mathrm{~cm}$ to ensure fiduciality in the CPR chamber

- remove photons which are in regions that are known to be inefficient due to hardware problems in the CPR

The philosophy of this method is very similar to that of the CES method described in section 7.4.1. This method also relies on a cut and the predicted efficiency of that cut for both signal and background, i.e., equations 7.11 and 7.14 are used again. This method relies on observing the rate at which prompt photons and background mesons convert in the solenoid as measured by the CPR detector (see section 3.3.4). A conversion is defined as occurring if the charge measured by the CPR, $Q_{C P R}$, is greater than $500 \mathrm{fC}$. This limit is much less than that from a minimum-ionising particle, but well above the typical noise fluctuations of $50 \mathrm{fC}$. A single prompt photon only has one 
chance of converting, where multiple photons from the decay of a neutral meson can convert. If the probability of a prompt photon converting is $P_{\gamma}=60 \%$, then the probability of a conversion signal from a meson such as a $\pi^{0}$ is:

$$
\begin{aligned}
P_{\pi^{0}} & =\text { probability that at least one photons converts } \\
& =1-\text { probability that neither photon converts } \\
& =1-\left(1-P_{\gamma}\right)^{2} \\
& =1-0.4^{2} \\
& =84 \%
\end{aligned}
$$

Additional effects are:

- CPR signals from the underlying event or electronic noise

The fractional rate at which an underlying event or electronic noise leaves a CPR signal which is falsely associated with a non-converted photon. A converted photon candidate is defined as one that has a CPR hit within $|\Delta x|<5 \mathrm{~cm}$ of a CES cluster.

- Inefficiencies in the $\mathrm{CPR}$

Inefficiencies in the CPR cause a photon candidate conversion to be undetected, causing the candidate to be classed as a "non-conversion" by mistake.

- known fraction of $\pi^{0}, K_{s}, \eta$ etc.

Figure 7.6 shows in detail the conversion probabilities for how $P_{c o n v}^{\gamma}$ and $P_{c o n v}^{Q C D}$ vary as a function of $E_{T}^{\mathrm{jet}}$. The dip in the background function at low $\mathrm{Pt}$ is due to the minimum momentum required for the photon to have a conversion signal survive to the CPR. 


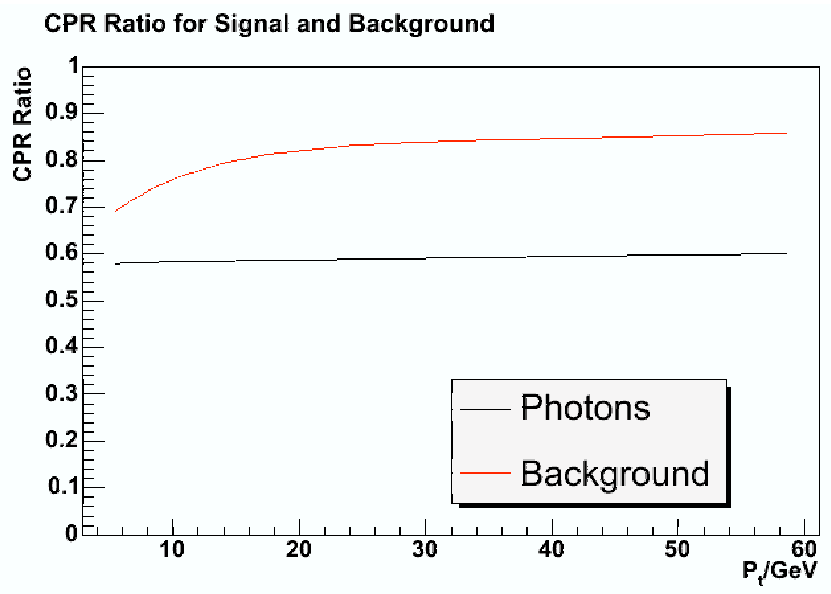

Figure 7.6: The conversion probabilities for signal prompt photons $\left(P_{c o n v}^{\gamma}\right)$, and background mesons $\left(P_{c o n v}^{Q C D}\right)$ as a function of $E_{T}$.

\subsubsection{Combining the three $F_{Q C D}$ Methods}

The three methods are compared in Figures 7.7 and 7.8. The fraction $F_{j e t}$ rises to 1 at $E_{T}^{\text {jet }}$ of $6 \mathrm{GeV}$, and falls exponentially to approximately $20 \%$ at $E_{T}^{\text {jet }}>40 \mathrm{GeV}$. All three methods are consistent within the systematic and statistical errors. As discussed in section 7.4.1, the CES and the Iso vs. $\chi^{2}$ methods breakdown at $E_{T}^{\gamma}>40 \mathrm{GeV}$ at which point only the CPR method is available. Between 15 and $20 \mathrm{GeV}$ the CPR method gives a value about 2-3 $\sigma$ higher than the other two methods. However the CES method is well tested in this region and considered more reliable at low $E_{T}^{\gamma}[99]$.

Whilst the CES and CPR method use the signal region and thus the same events, the Iso-CES method uses events outside the signal region to extrapolate into the signal region. Hence, there is no statistical correlation between the two CES methods. For the final estimate of $F_{\text {jet }}$, the error 


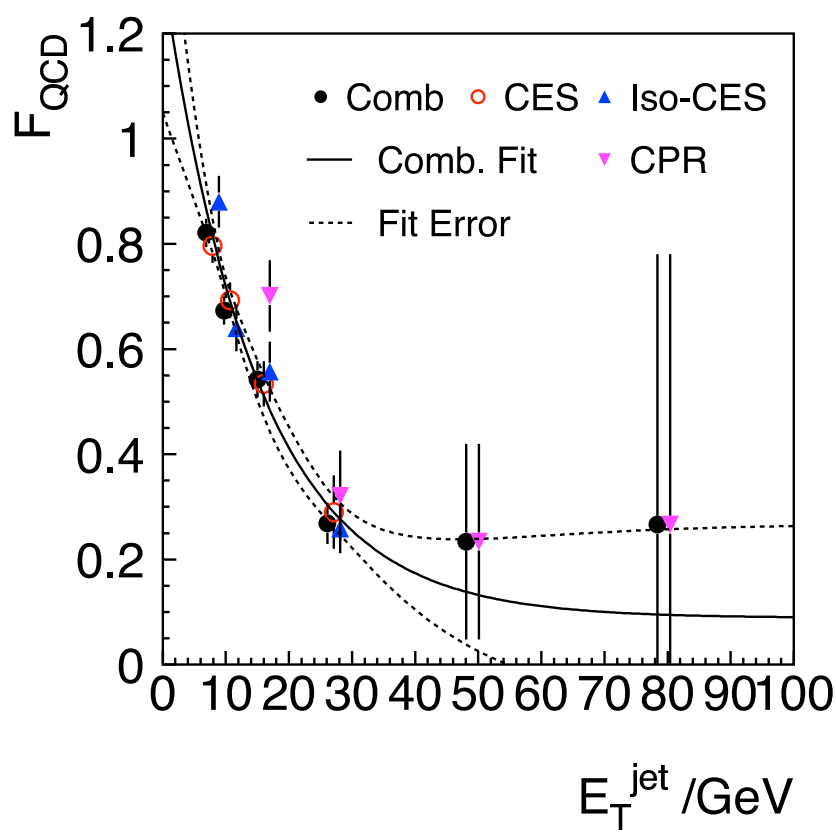

Figure 7.7: $F_{\text {jet }}$ as a function of $E_{T}^{\text {jet }}$, as measured using the three independent methods for the 345th jet sample. "Comb" denotes the combination of the three methods as discussed in the text. The data are compared to the final fit (solid line) of the average of the "Iso-CES" and "CES" for $E_{T}^{\gamma}<40 \mathrm{GeV}$ and the $\mathrm{CPR}$ for $E_{T}^{\gamma}>40 \mathrm{GeV}$. The upper and lower errors on the fit are also shown (dashed lines).

weighted average of the CES and Iso-CES method for $E_{T}^{\gamma}<40 \mathrm{GeV}$ and the CPR method for $E_{T}^{\gamma}>40 \mathrm{GeV}$ is used.

This combined $F_{j e t}$ estimate is fitted with a function of form

$$
e^{-a \cdot x+b}+c
$$

The fit is shown in figure 7.7 and the fitted parameters are $a=0.207, b=$ 0.0668 and $c=0.0888$. The systematic error associated with the $F_{j e t}$ is taken to be the statistical error on the final fit, also shown in figure 7.7. All data 


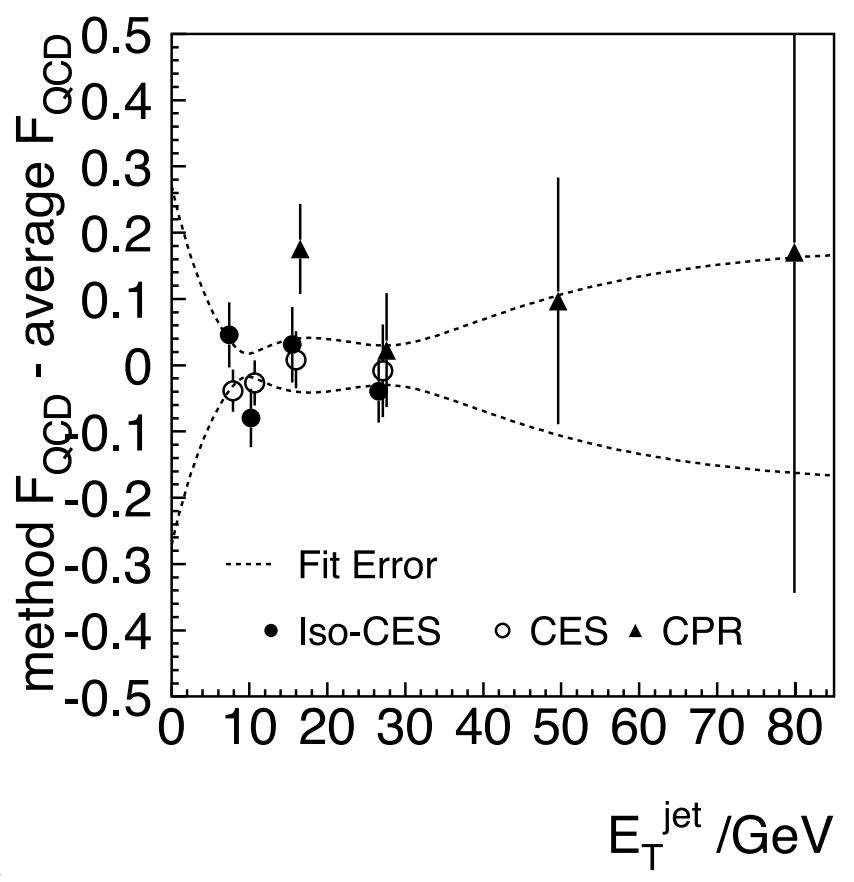

Figure 7.8: Shown is the ratio of the $F_{\text {jet }}$ determinations (closed point: Iso vs CES, open points: CES, triangles: CPR) to the final value used and the systematic error (shaded ares) and the statistical errors of the individual methods.

are consistent within the statistical error on the fit. It ranges from about $5 \%$ at low $E_{T}^{\gamma}$ to $15 \%$ at high $E_{T}^{\gamma}$. This corresponds to a fractional error on $F_{\text {jet }}$ of about $7 \%$ at low $E_{T}^{\gamma}$ and $250 \%$ at high $E_{T}^{\gamma}$. This error is propagated to the total systematic error on $F_{j e t}$.

\subsection{The True Fake Probability}

The final value of $P_{\text {jet } \rightarrow \gamma}\left(E_{T}^{\text {jet }}\right)=P_{\text {raw }}\left(E_{T}^{\text {jet }}\right) \times F_{\text {jet }}$ is shown in Figure 7.9. The fit has an exponential form:

$$
e^{-a \cdot x+b}+c
$$


with parameters $\mathrm{a}=0.1526, \mathrm{~b}=2.3969$ and $\mathrm{c}=0.40369$. The systematic error is also shown. It is discussed in detail in section 7.7.
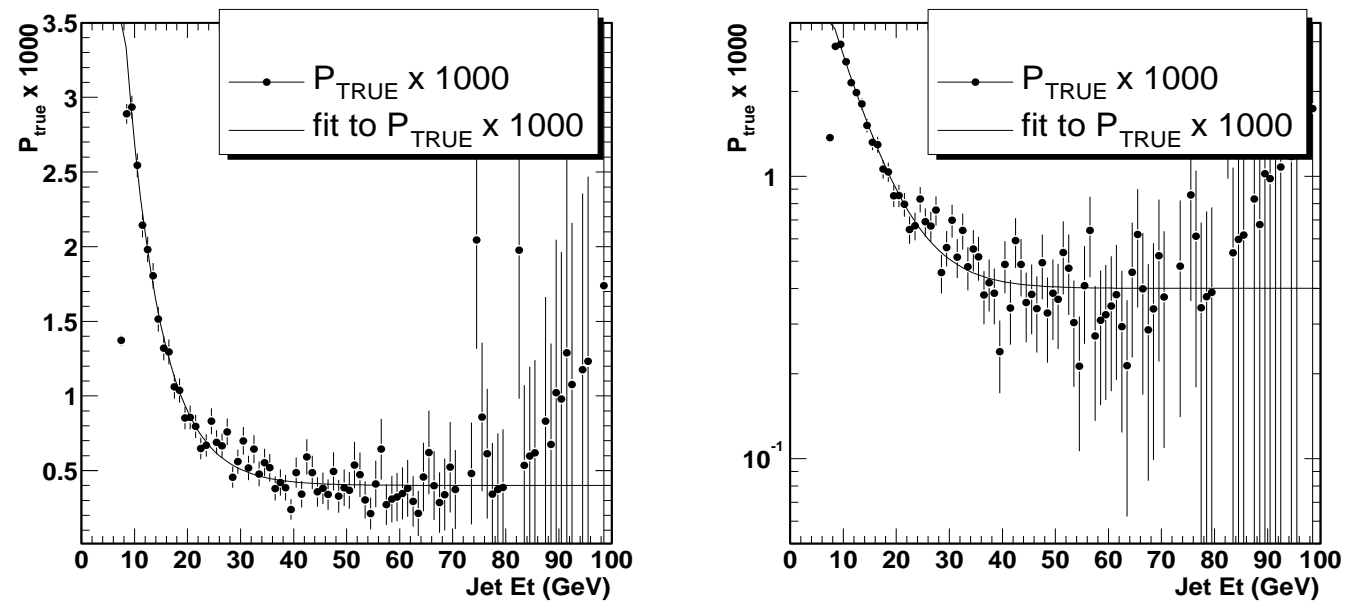

Figure 7.9: The corrected "true" fake rate $P_{\text {jet } \rightarrow \gamma}\left(E_{T}^{\text {jet }}\right)$ for the 345 th jet sample. The result is shown on a linear (left) and logarithmic scale (right).

\subsection{Fragmentation}

From section 7.5, the fraction $P_{\text {jet } \rightarrow \gamma}\left(E_{T}^{\text {jet }}\right)$ has been derived. However, in order to equate $E_{T}^{j e t}$ to $E_{T}^{\gamma}$, to obtain a prediction for the fake photon contribution from QCD jets as given by equation 7.1 , the term $z\left(E_{T}^{\text {jet }}, E_{T}^{\gamma}\right)$ must be evaluated. This term, known as the fragmentation gives the probability that a jet of $E_{T}^{\text {jet }}$ will be measured as $E_{T}^{\gamma}$.

Figure 7.10 shows the ratio of $E_{T}^{\gamma}$ and $E_{T}^{\text {jet }}$ for photon candidates. A gaussian is used to fit this ratio.

Integrating over all $E_{T}^{j e t}$, the mean and sigma of the fragmentation are $\bar{Z}=0.934 \pm 0.001$ and $\sigma_{Z}=0.0428 \pm 0.0008$ respectively. Figure 7.11 shows 


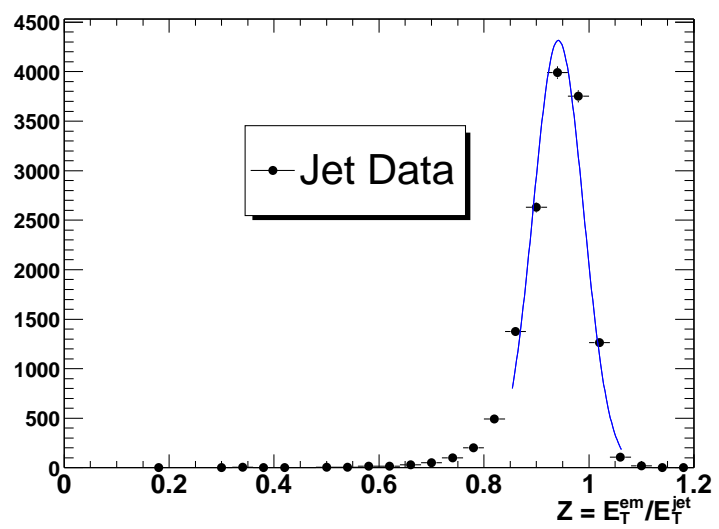

Figure 7.10: The distribution of $Z=E_{T}^{\gamma} / E_{T}^{\text {jet }}$ in the jet triggered data samples. Shown is a Gaussian fit to the distribution.

the mean values of the Gaussian fits and the resolutions as function of $E_{T}$. The mean value increases by about $2 \%$ between 7 and $40 \mathrm{GeV}$ whilst the resolution decreases by about $1 \%$ over that range. A $2 \%$ systematic error is thus assigned. This is discussed in more detail in section 7.7.4.

\subsubsection{Application of Fake Rate}

The fake rate is applied to jets in $Z^{0}+$ jet events, where the jet has $|\eta|<1$. and is separated from the leptons by $\Delta R>0.7$.

\subsection{Systematic Studies}

The following section describes the sources of systematic error for the fake rate method. The errors considered are:

- the statistical error on $F_{j e t}$ fit (see section 7.4.4); 

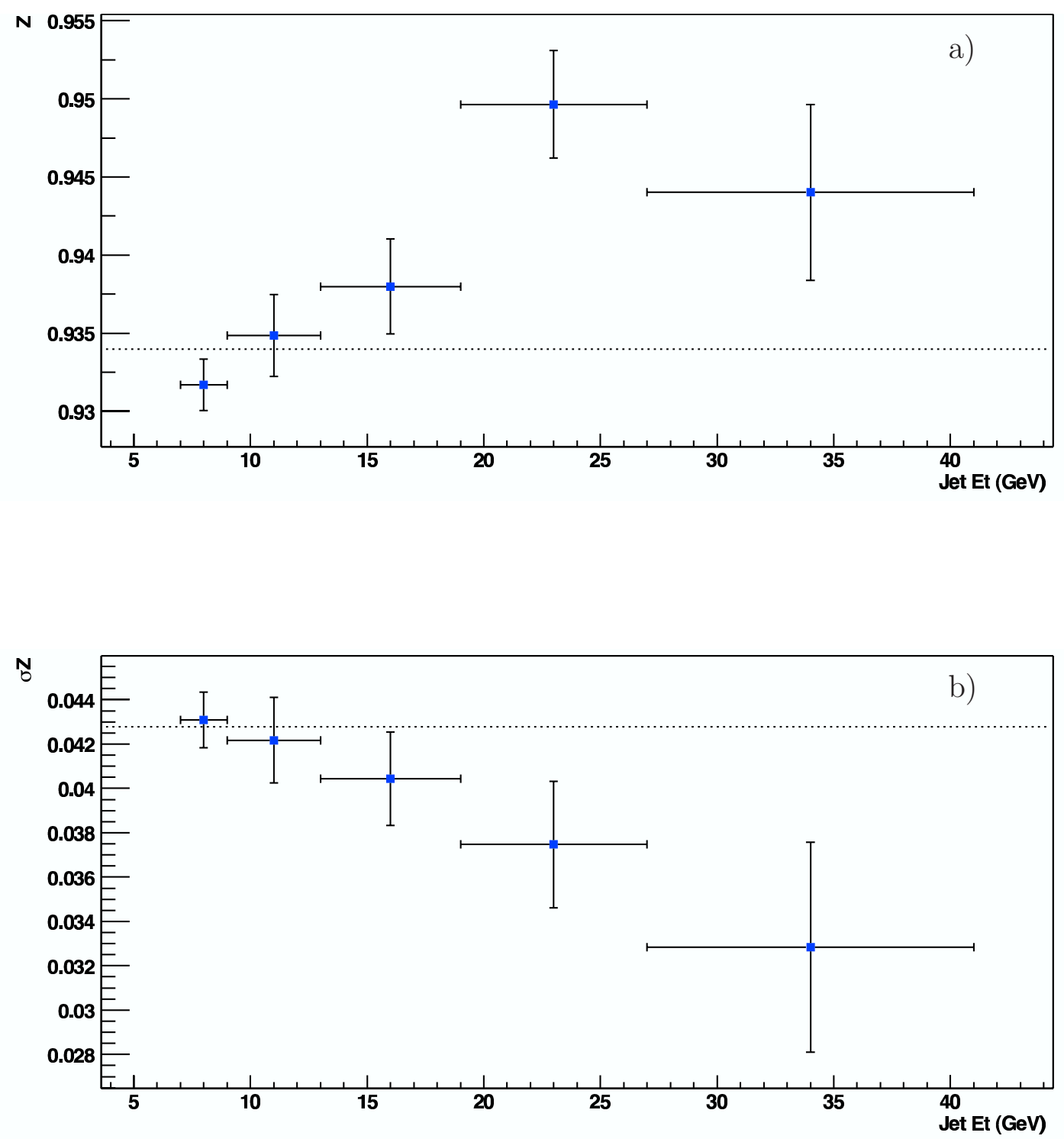

Figure 7.11: Dependence of a) the mean value and b) the resolution of $Z^{0}$ on $E_{T}^{j e t}$. The dashed lines indicates the average values of the full sample. 
- the difference between the fakerate obtained by the 2 nd rather than the 345 th jet;

- the difference in fragmentation between a generic jet and one with a leading hard $\pi^{0}$, estimated by measuring the fake rate with respect to a loose electromagnetic object rather than with respect to a jet;

- the parameterization of the fragmentation;

- the effect due to possible differences in the quark/gluon mixture between jets in $Z^{0}$ events and the 3rd jet in the JET triggered samples.

\subsection{1 $\quad F_{j e t}:$ See section 7.4 .4}

The statistical error on the $F_{j e t}$ is approximately $10 \%$ for $E_{T}^{\gamma}<30 \mathrm{GeV}$, increasing to $200 \%$ at $70 \mathrm{GeV}$.

\subsubsection{Comparison of 2 nd to 345 th jet}

Using the 2nd jet is a powerful test of the method. It has properties which are very different to the 345th. These differences are summarised below.

- The 2nd jet has a different $E_{T}^{\text {jet }}$ spectrum as shown in Figure 7.1. It has a harder spectrum and extends to higher values of $E_{T}^{\text {jet }}$.

- The 2 nd jet has a different prompt photon fraction. Figure 7.12 shows the processes contributing to prompt photon production:

- The LO process ( $\mathrm{a}$ and $\mathrm{b}$ ) will usually result in the photon being classified as 1st jet. In principle the jet and $\gamma$ will have the same 
$E_{T}$. However, single isolated objects such as photons and electrons have a well measured energy in the calorimeter with a calorimeter response of about 100\%. A generic jet with many particles, only has a calorimeter response of about $70 \%$, due to mismeasurement and energy loss down the calorimeter cracks. This means that the 2nd jet is most likely not be the photon.

- In process c) the photon is radiated from one of the quarks and will thus usually be the 3rd "jet". Thus photons being classified as 2 nd jet are suppressed.

This results in a large difference in $P_{\text {raw }}\left(E_{T}^{\text {jet }}\right)$ and $F_{\text {jet }}$ between the 2nd and 345th jet but should not result in any significant difference for $P_{\text {jet } \rightarrow \gamma}\left(E_{T}^{\text {jet }}\right)=P_{\text {raw }}\left(E_{T}^{\text {jet }}\right) \times F_{\text {jet }}$ if the procedure works correctly.

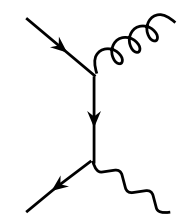

a)

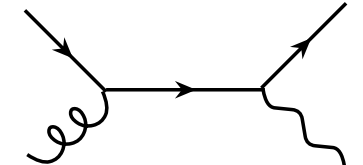

b)

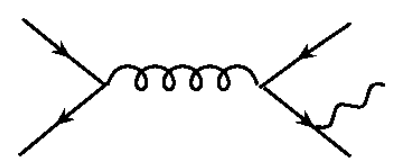

c)

Figure 7.12: Feynman diagrams for photon production. a) and b) show the prompt photon production diagrams and c) a final state radiation example diagram.

- the quark and gluon fractions are different compared to the 345th jet, in the high $E_{T}^{j e t}$ region. This can be seen by comparing figures 7.23 and 7.13 for the 345 and 2nd jets in PYTHIA jet MC respectively. 


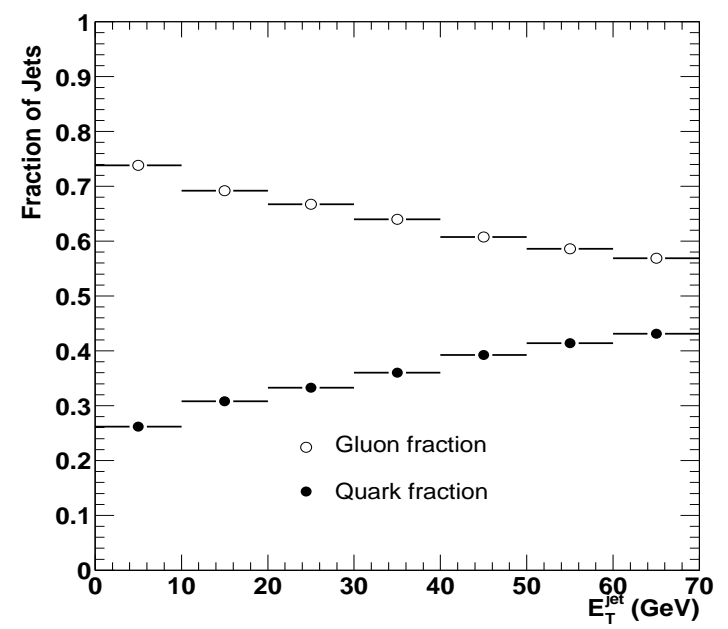

Figure 7.13: Fractional contribution of quark and gluon jets for the 2nd jet in the PYTHIA jet MC.

The measurements of $P_{\text {raw }}\left(E_{T}^{\text {jet }}\right), F_{\text {jet }}$ and $P_{\text {jet } \rightarrow \gamma}\left(E_{T}^{\text {jet }}\right)$ are shown for the 2nd jet in Figures 7.14, 7.15 and 7.16 respectively.

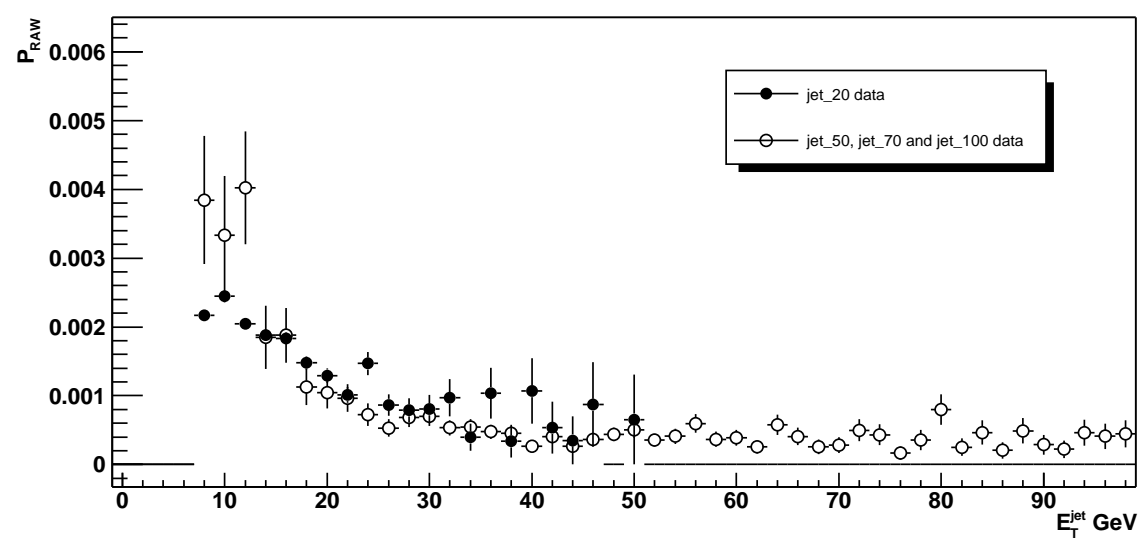

Figure 7.14: $P_{\text {raw }}\left(E_{T}^{\text {jet }}\right)$ for 2 nd jet sample versus $E_{T}^{\text {jet }}$ 


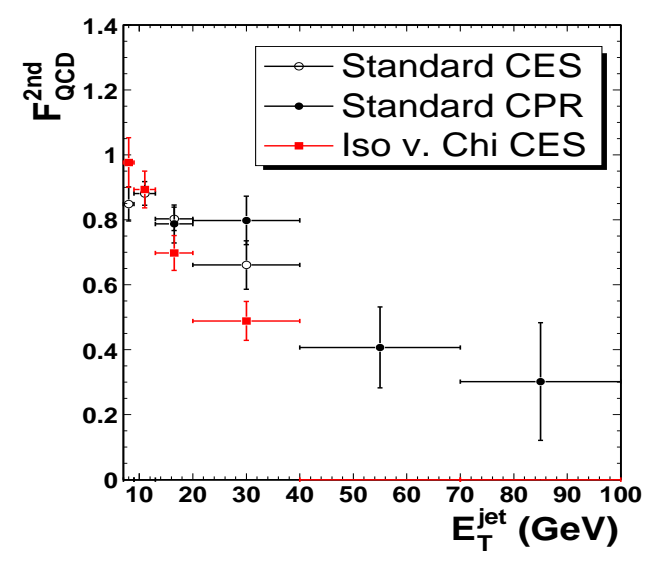

Figure 7.15: $F_{\text {jet }}$ for 2nd jet sample versus $E_{T}^{\text {jet }}$

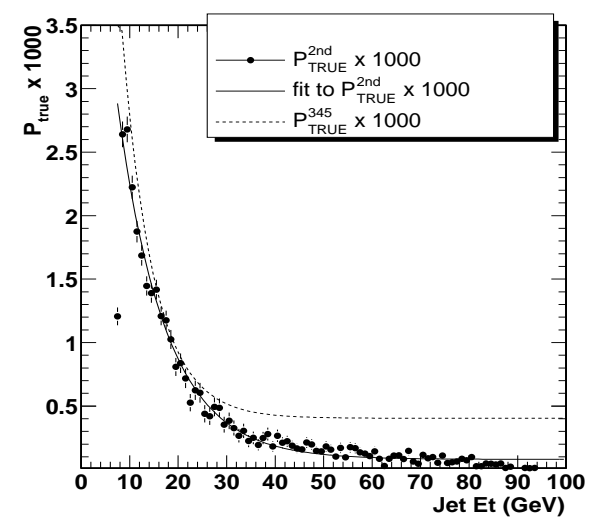

Figure 7.16: $\quad P_{\text {jet } \rightarrow \gamma}\left(E_{T}^{\text {jet }}\right)$ for 2 nd jet sample versus $E_{T}^{\text {jet }}$. Shown for comparison is the function for $P_{\text {jet } \rightarrow \gamma}\left(E_{T}^{\text {jet }}\right)$ calculated in the 345th jet sample.

Both the raw fake rate and $F_{j e t}$ values differ significantly between the 2nd and 345th jet due to the jet ordering bias described above. For example, at $15 \mathrm{GeV}$ the raw rate is about $0.2 \%$ for the 2 nd but $0.4 \%$ for the 345 th. The fraction $F_{j e t}$ is larger for the 2 nd jet sample. At approximately $25 \mathrm{GeV}$ it is about $30 \%$ for the 345 th jet whilst it is around $60 \%$ for the 2 nd jet. The ratio of the 2 nd to the 345 th $P_{\text {jet } \rightarrow \gamma}\left(E_{T}^{\text {jet }}\right)$ predictions is taken as a systematic error, in the fake rate calculation, since it represents an uncertainty on the procedure. This systematic error is shown as a function of $E_{T}^{\text {jet }}$ in figure $7.25 \mathrm{~b}$. It is about $10 \%$ at low $E_{T}^{\text {jet }}$ and about $50 \%$ at $E_{T}^{\text {jet }} \approx 40 \mathrm{GeV}$. 


\subsubsection{EM based fake rate}

One of the concerns in the fake rate measurement is the fragmentation of a jet into a photon candidate and how to translate $E_{T}^{\text {jet }}$ to $E_{T}^{\gamma}$. This uncertainty can be removed by using an electromagnetic energy cluster or EMobject (see section 4.2.3) as the denominator instead of a jet. This fake rate can then be applied to $Z^{0}+$ EMobject events, ie, the equation 7.1 is rewritten using $E_{T}^{\mathrm{EM}}$ instead of $E_{T}^{\text {jet }}$ and $E_{T}^{\mathrm{EM}}=E_{T}^{\gamma}$ :

$$
N_{B G}\left(E_{T}^{\gamma}\right)=\int_{0}^{N_{E M}(Z)} P_{\mathrm{EM} \rightarrow \gamma}^{Z}\left(E_{T}^{\mathrm{EM}}\right) \cdot \mathrm{d} N_{Z} / \mathrm{d} E_{T}^{\mathrm{EM}} \cdot \mathrm{d} E_{T}^{\mathrm{EM}} .
$$

Similarly, equations 7.3 and 7.5 are rewritten:

$$
\begin{aligned}
F_{E M} & =\frac{N_{E M \rightarrow \gamma}}{N_{\gamma-\text { candidate }}}=1-F_{\gamma} \\
P_{\mathrm{EM} \rightarrow \gamma}^{j}\left(E_{T}^{\mathrm{EM}}\right) & \equiv P_{\text {raw }}\left(E_{T}^{\mathrm{EM}}\right) \times F_{E M} .
\end{aligned}
$$

The EM based fake rate is measured from EMobjects in the JET_20, JET_50, JET_70 and JET_100 triggered samples. An EMobject enters the denominator if it is found to be within $|\eta|<1.1$ and the event has a good vertex. Because EMobjects contain only calorimeter information, they do not distinguish between electrons and photons. In order to exclude electrons, EMobjects entering the denominator are required to pass the $N_{\text {track }}$ cut of the photon, i.e. EMobjects are only included in the denominator, if they have either no associated track or one track with $P_{T}<1+0.005 \cdot E_{T}$.

The numerator for the raw EM fake rate is the sub-sample of EMobjects from the denominator, which also pass the selection criteria in table 6.1. The raw fake rate is plotted for EMobjects which are matched to the trigger object $(\Delta R<0.4)$ and those which are not, in figure 7.17. A strong trigger bias 
is clearly apparent, with peaks in the raw fake rate for the trigger object, at 20, 50, 70 and $100 \mathrm{GeV}$. Any EMobject associated (within $\Delta R<0.4$ of the trigger jet) is excluded from both the numerator and denominator. $P_{\text {raw }}\left(E_{T}^{\mathrm{EM}}\right), F_{E M}$ and $P_{\mathrm{EM} \rightarrow \gamma}^{j}\left(E_{T}^{\mathrm{EM}}\right)$ are shown in Figures $7.17,7.18$ and 7.19 respectively. Both $P_{\text {raw }}\left(E_{T}^{\mathrm{EM}}\right)$ and $P_{\mathrm{EM} \rightarrow \gamma}^{j}\left(E_{T}^{\mathrm{EM}}\right)$ are clearly different from the jet based fake rate. They are not directly comparable since they apply to different objects. $F_{E M}$ is similar in form to that of $F_{j e t}$ for the jet based method, but here it is determined as function of $E_{T}^{\mathrm{EM}}$ instead of $E_{T}^{\mathrm{jet}}$. The EM fake rate is applied to all $Z^{0}+$ EMobject events where the EMobject has $|\eta|<1.1$, is separated from the leptons by $\Delta R>0.7$ and passes the $N_{\text {track }}$ cut of the photon selection.

Both the "jet based" and the "EM" based fake rates are applied to their respective samples to predict the background to $Z^{0} \gamma$ and $W^{ \pm} \gamma$ events. $W^{ \pm} \gamma$ events were selected using the $W^{ \pm}$selection described in section 6.2.1 plus a photon candidate passing the selection cuts of table 6.1, separated from the lepton by $\Delta R>0.7$. The background predictions, compared in Figure 7.20, agree to within $20 \%$. This difference is taken as a systematic error.

\subsubsection{Effect of Varying Fragmentation and Smear}

In order to investigate the effect of scaling and smearing $E_{T}^{j e t}$ to $E_{T}^{\gamma}$, when applying the fake rate, the values of the fragmentation and smear are changed, and the new resultant prediction of the fake photon spectrum in the JET_20, JET_50, JET_70 and JET_100 jet data samples are compared. The fraction of the "varied parameter" prediction to that of the "default parameter" prediction is measured to estimate the systematic error. The fragmentation was 


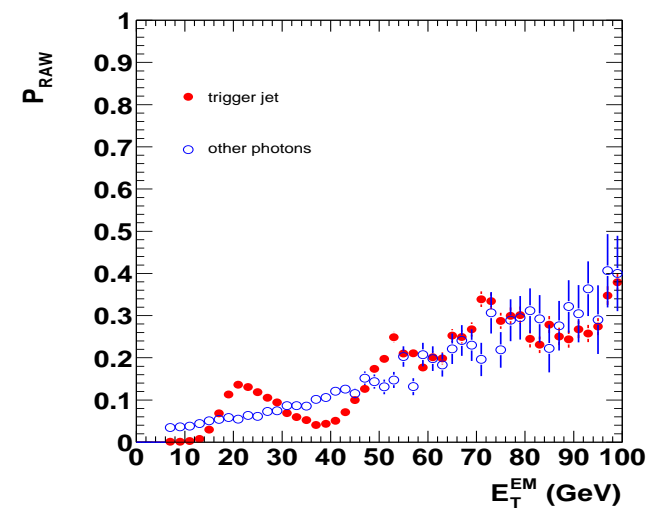

Figure 7.17: $P_{\text {raw }}\left(E_{T}^{\text {jet }}\right)$ for EM objects versus $E_{T}^{E M}$

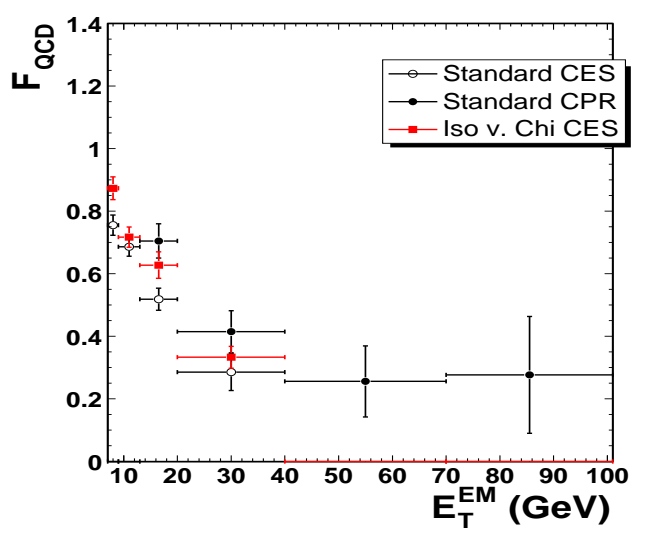

Figure 7.18: $F_{E M}$ for EM objects versus $E_{T}^{E M}$

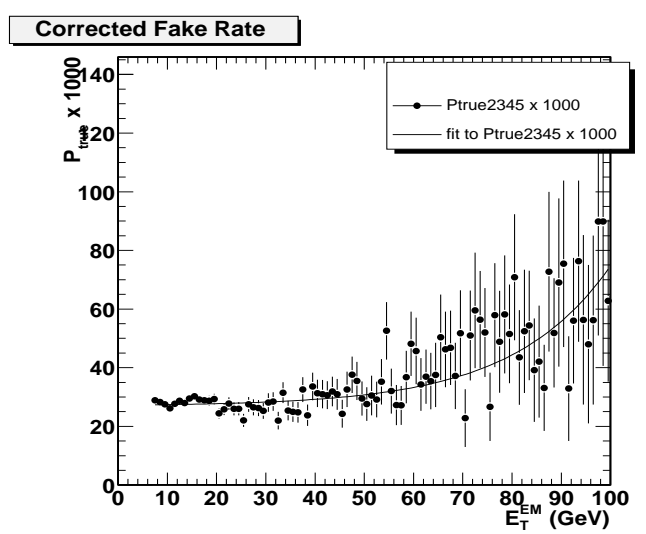

Figure 7.19: $P_{\text {jet } \rightarrow \gamma}\left(E_{T}^{\text {jet }}\right)$ for EM objects versus $E_{T}^{E M}$. 

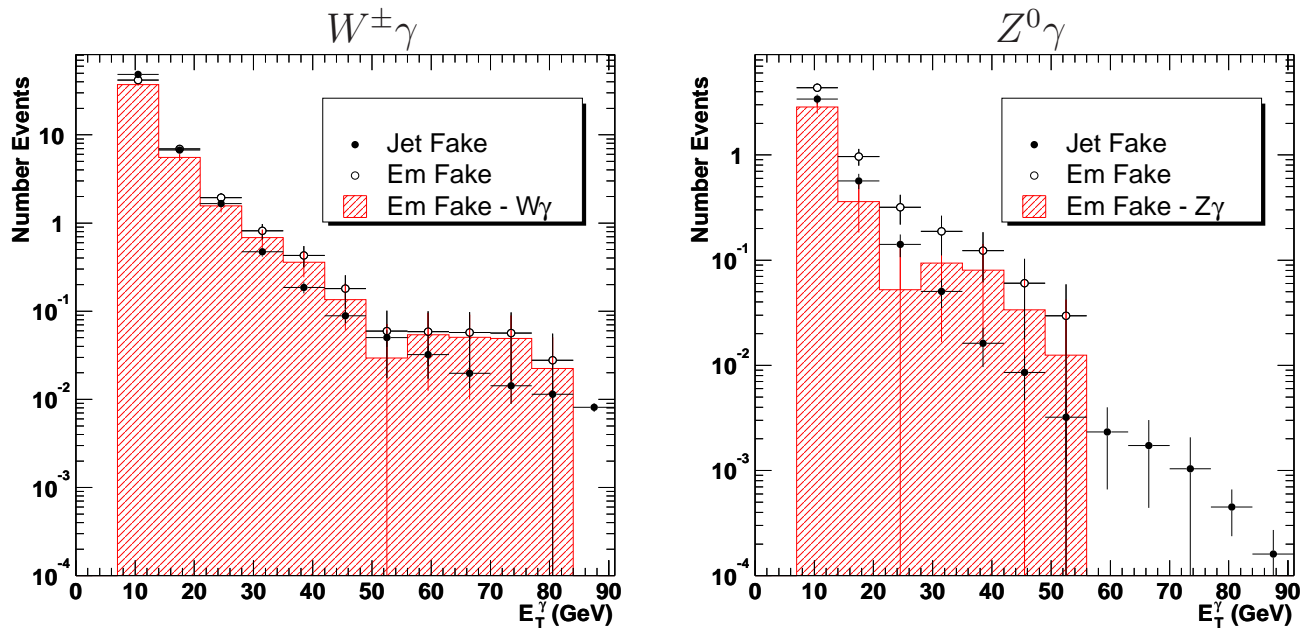

Figure 7.20: The QCD predicted background for the $W^{ \pm} \gamma$ (left) and $Z^{0} \gamma$ (right) samples using the jet based fake rate method (closed points) and the EM fake rate (open points). Also shown is the EM fake rate prediction after subtracting the expected contaminations from $W \gamma$ and $Z^{0} \gamma$ signal (histogram). 
altered by $\pm 3 \%$. The corresponding systematic error, shown in figure $7.25 \mathrm{~d}$, rises from about $10 \%$ at low $E_{T}^{\text {jet }}$ to $60 \%$ at high $E_{T}^{\text {jet }}$. The effect of changing the smearing from 0.034 to 0.044 , on the fake rate prediction was found to be less than $2 \%$ and thus was considered negligible.

\subsubsection{Effect of different Quark/Gluon mixtures}

The aim is to measure a fake rate in a jet triggered sample and then apply it to the $Z^{0}+$ jet sample. This will only be valid if the jet in the $Z^{0}+$ jet sample has the sample properties as the 345th jets from the jet triggered sample, i.e. in equation 7.1: $P_{\text {jet } \rightarrow \gamma}^{j}\left(E_{T}^{\text {jet }}\right)=P_{\text {jet } \rightarrow \gamma}^{Z}\left(E_{T}^{\text {jet }}\right)$. Figure 7.1 clearly shows these two samples have the same $E_{T}^{\text {jet }}$ spectrum. It is also important to consider the source of these jets, whether they originate from the hadronisation of a quark or a gluon. Gluonic jets tend to have a softer fragmentation function than quark jets, and hence a higher particle multiplicity [95].

Using truth information available from jet MC samples, jets can be matched in $\Delta R$ to hard quarks and gluons emitted in the hard interaction. These quarks and gluons then fragment producing a hadronic shower including mesons, which can occasionally pass the photon selection. The matching criteria are:

- if jet is matched to within $\Delta R<0.7$ of a quark (gluon) emitted in hard interaction, the jet is labelled as a "quark" ("gluon") jet.

- if a jet can be matched to more than one parton, it is assumed to come from the parton closest in energy to the truth level energy of the jet.

The $P_{T}$ distribution of the highest $P_{T} \pi^{0}$ in PYTHIA jet $\mathrm{MC}$ is shown in Figure 7.21, for quark and gluon jets seperately. 
$P_{\text {jet } \rightarrow \gamma}^{j}\left(E_{T}^{\text {jet }}\right)$ is calculated in jet MC samples using truth information to remove prompt photons on a per event basis. Jets only enter the numerator of $P_{\text {jet } \rightarrow \gamma}^{j}\left(E_{T}^{\text {jet }}\right)$ if the jets were matched to within $\Delta R<0.05$ of a $\gamma$ which has come from the decay of a $\pi^{0}, \eta$ or $K_{s}$.

Figure 7.22 shows that the $P_{\text {jet } \rightarrow \gamma}^{j}\left(E_{T}^{\text {jet }}\right)$ for quark jets is considerably greater than that for gluon jets.

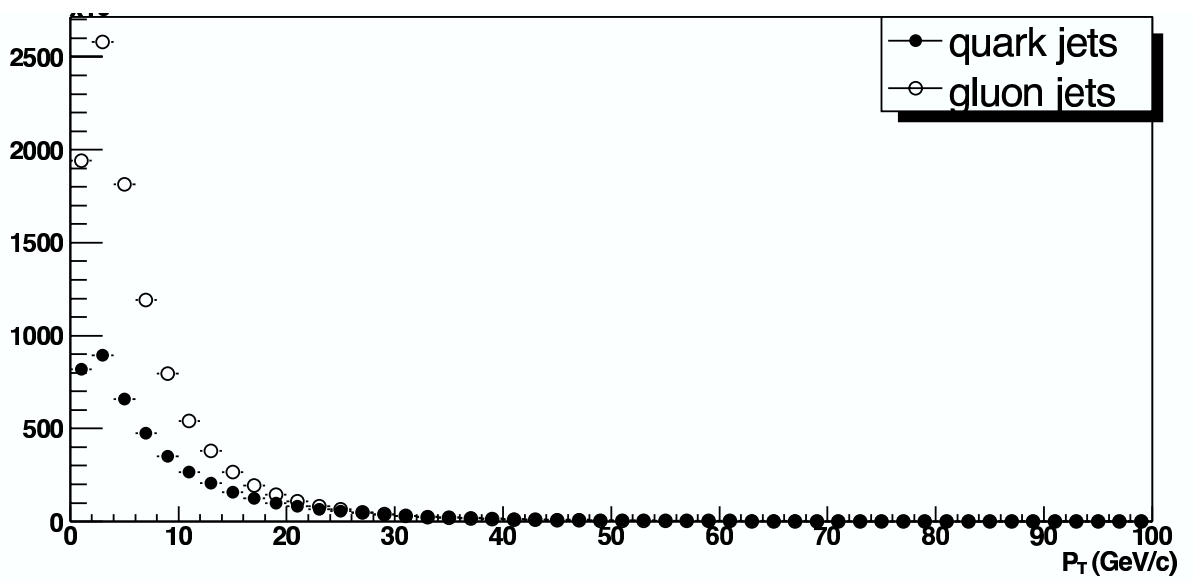

Figure 7.21: $P_{T}$ distribution of highest $P_{T} \pi^{0}$ in gluon and quark jets in PYTHIA jet MC. Although there are more gluon jets present, their $P_{T}$ spectrum is much softer.

In order to test the effect of any discrepancy in the quark-gluon composition between the jet sample and $Z^{0}+$ jets, the fraction of quark and gluon jets, out of the total number of jets successfully identified, are compared between an ALPGEN[80] $Z^{0}+1$ jet and a PYTHIA[81] dijet Monte Carlo sample, and shown versus $E_{T}^{\text {jet}}$, in Figure 7.23.

At low $E_{T}^{\text {jet }}$ the two MC samples predict very similar gluon jet fractions $\left(F_{g}\right)$ of about $70 \%$ but with increasing $E_{T}^{\text {jet }}$, the quark jet becomes dominant 


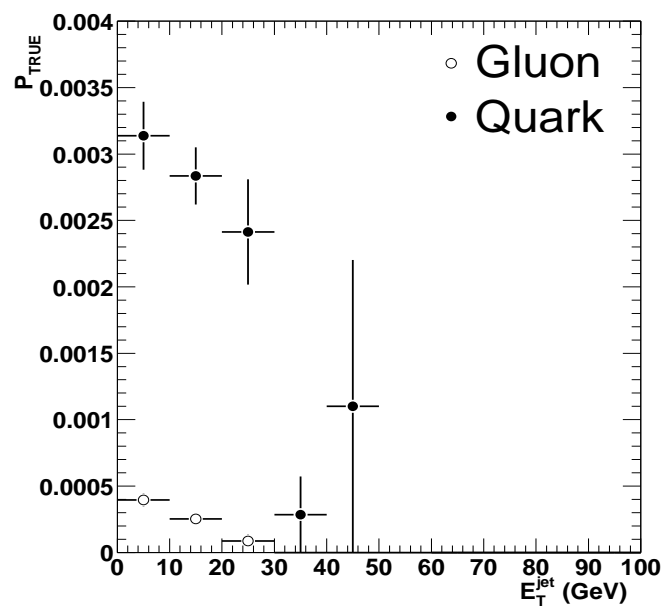

Figure 7.22: Fake rate for quark and gluon jets for jets containing a hard $\pi^{0}$ or $\eta$ in HERWIG jet MC.
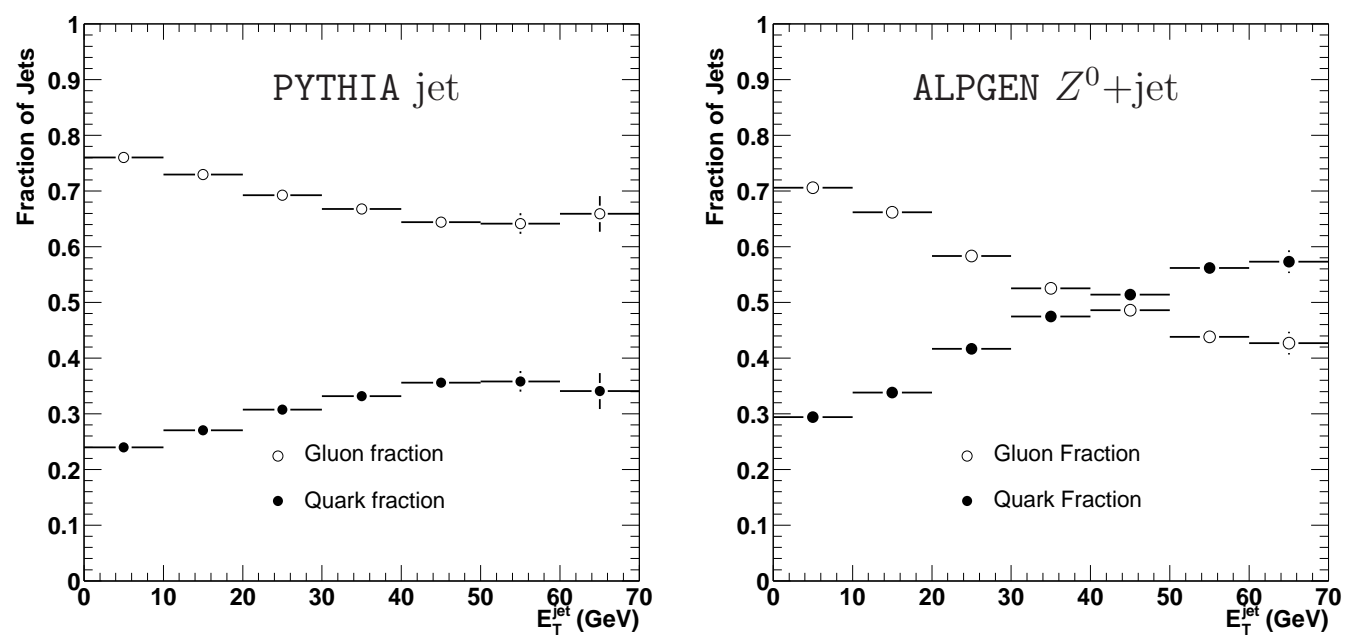

Figure 7.23: Fractional contribution of quark and gluon jets in the PYTHIA dijet MC (left) and the ALPGEN $Z+1$ jet MC. 
in the $Z^{0}+1$ jet $\mathrm{MC}$ : at $E_{T}^{\text {jet }}=70 \mathrm{GeV}$ the fraction of quark jets $\left(F_{q}\right)$ is about $55 \%$ for the $Z^{0}+1$ jet sample and only $35 \%$ for the jet sample. The jet based fake rate, $P_{\text {jet } \rightarrow \gamma}^{j}\left(E_{T}^{\text {jet }}\right)$ can be considered a sum of $P_{\mathrm{q} \rightarrow \gamma}^{j}\left(E_{T}^{\text {jet }}\right)$ and $P_{\mathrm{g} \rightarrow \gamma}^{j}\left(E_{T}^{\mathrm{jet}}\right)$, weighted by their relative fractions:

$$
P_{\mathrm{jet} \rightarrow \gamma}^{j}\left(E_{T}^{\mathrm{jet}}\right)=P_{\mathrm{q} \rightarrow \gamma}^{j}\left(E_{T}^{\mathrm{jet}}\right) \cdot F_{q}+P_{\mathrm{g} \rightarrow \gamma}^{j}\left(E_{T}^{\mathrm{jet}}\right) \cdot F_{g}
$$

At a jet energy of $20 \mathrm{GeV}, F_{g}=0.35$ and $F_{q}=0.65\left(F_{g}=0.45\right.$ and $\left.F_{q}=0.55\right)$ for the dijet $\left(Z^{0}+\right.$ jet $)$ sample. From Figure 7.22 , the fake rate is $P_{\mathrm{q} \rightarrow \gamma}^{j}\left(E_{T}^{\mathrm{jet}}\right)=$ $25 \times 10^{-4}$ and $P_{\mathrm{g} \rightarrow \gamma}^{j}\left(E_{T}^{\mathrm{jet}}\right)=1 \times 10^{-4}$ for quark and gluon jets respectvely. Using these values in equation 7.21 results in a prediction of the difference in $P_{\text {jet } \rightarrow \gamma}^{j}\left(E_{T}^{\text {jet }}\right)$ between the $Z^{0}+$ jet and dijet data samples of $20 \%$, at $E_{T}^{\text {jet }}=20$ $\mathrm{GeV}$ :

$$
\begin{aligned}
\text { dijet } & : \quad P_{\text {jet } \rightarrow \gamma}\left(E_{T}^{\text {jet }}\right)=0.0025 \cdot 0.35+0.0001 \cdot 0.65=0.0009 \\
Z^{0}+1 \text { jet } & : \quad P_{\text {jet } \rightarrow \gamma}\left(E_{T}^{\text {jet }}\right)=0.0025 \cdot 0.45+0.0001 \cdot 0.55=0.0011
\end{aligned}
$$

The resultant systematic error for this effect is shown in figure 7.26. At the highest $E_{T}^{\text {jet }}$ values of $70 \mathrm{GeV}$, the disparity in quark/gluon content could result in a difference of up to $100 \%$. This is however an over estimate. Higher (NLO) order processes typically tend to increase the gluon fraction and would thus reduce the difference between the $Z^{0}+$ jet and the dijet MC samples. No correction is applied for this effect since the MC prediction on the individual quark and gluon fake rates is unreliable. This is seen in Figure 7.24. Here, $P_{\text {jet } \rightarrow \gamma}^{j}\left(E_{T}^{\text {jet }}\right)$ is plotted, as measured in two different dijet data samples using the method set out in section 7.4 to remove the true photon contamination. The prediction is statistically limited, but it shows a clear discrepancy between the data and the two simulations. Also HERWIG and PYTHIA differ significantly. 


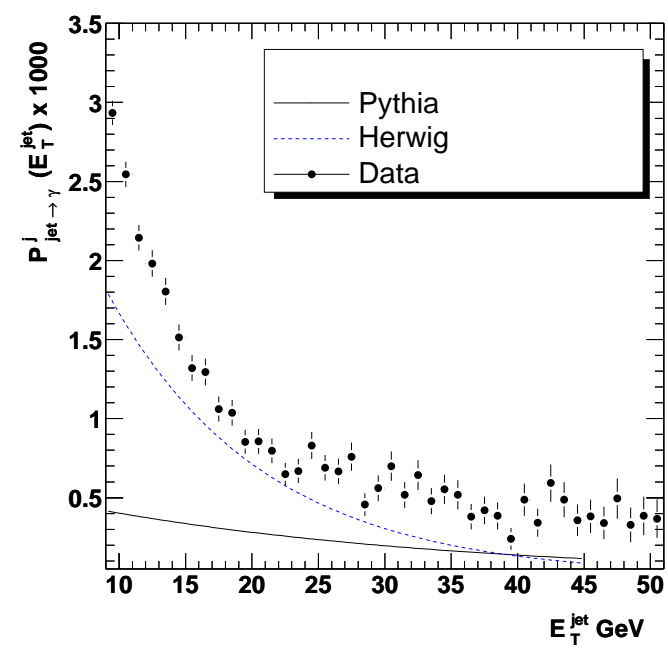

Figure 7.24: $P_{\text {jet } \rightarrow \gamma}^{j}\left(E_{T}^{\text {jet }}\right)$ as measured in PYTHIA and HERWIG MC samples. For Comparison purposes, $P_{\text {jet } \rightarrow \gamma}^{j}\left(E_{T}^{\text {jet }}\right)$ measured from the 345 th data sample is also shown. The MC samples have approximately a $40 \%$ statistical error.

\subsubsection{Conclusion of Systematic Studies}

The five systematic errors are shown in Figures 7.25 and 7.26 as a function of $E_{T}^{\text {jet }}$. These errors are treated as uncorrelated and hence the total systematic error is the quadratic sum of the five individual errors. This total error is shown in the Figure 7.26. The total systematic error increases with $E_{T}^{\text {jet }}$ from $20 \%$ at low $E_{T}^{\text {jet }}$ to $200 \%$ at high $E_{T}^{\text {jet }}$. The dominant error is from the deviation of results from the various $F_{j e t}$ methods as described in section 7.4. Other studies and sources of possible systematic errors are described in section A.3.

The fit to the total systematic error, upper and lower bands have the exponential form:

$$
e^{a \cdot x+b}+c .
$$


The upper error band has parameters $\mathrm{a}=2.43 \times 10^{-2}, \mathrm{~b}=-0.833$ and $\mathrm{c}=0.692$ and the lower error band has parameters $\mathrm{a}=-5.96 \times 10^{-4}, \mathrm{~b}=3.47$ and $\mathrm{c}=-31.3$. The systematic error shown is discussed in section 7.7.
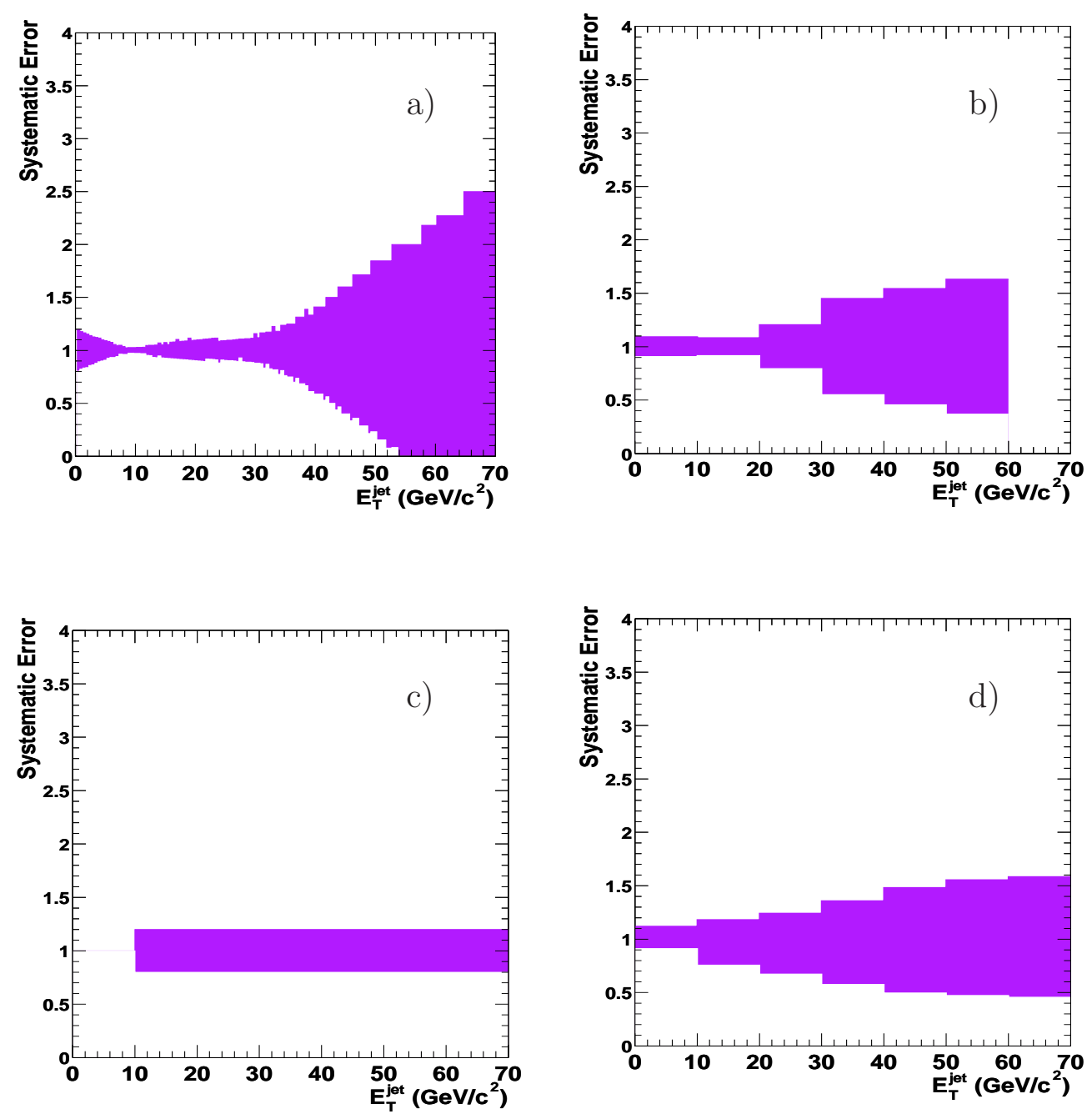

Figure 7.25: Fractional systematic error on the fake rate due to: a) the method for statistical precision on $F_{j e t}$, b) the fake rate determined from 2nd jet sample, c) the fake rate determined from using EMobjects as the denominator, d) the fake rate determined from varying the fragmentation. 

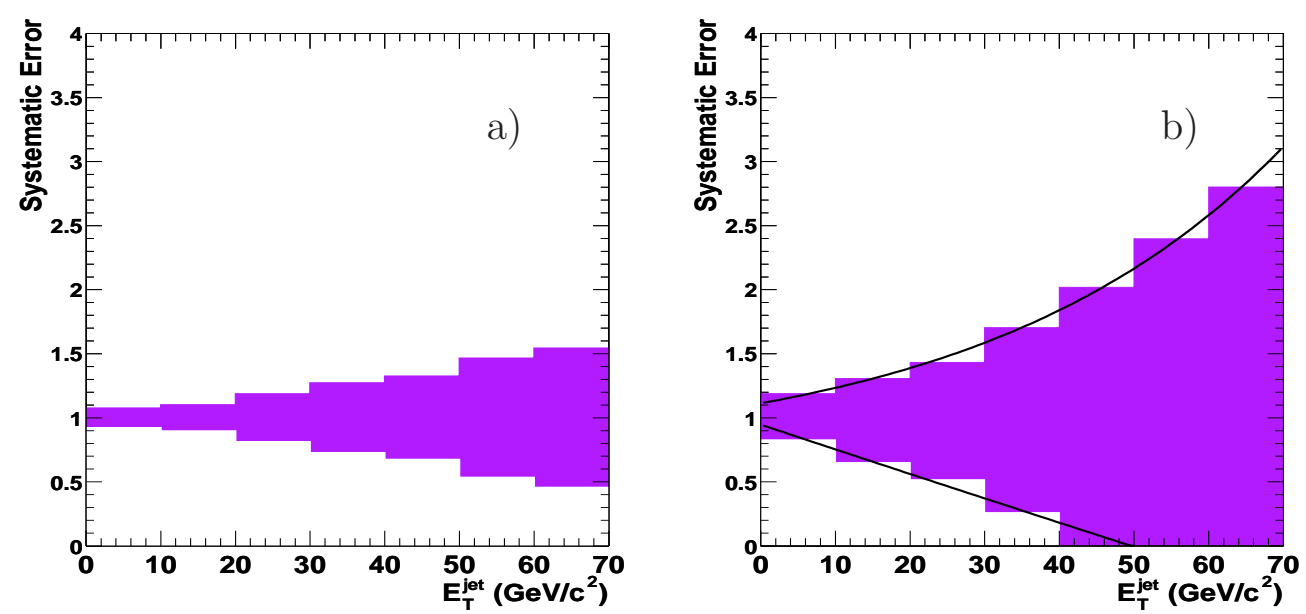

Figure 7.26: Figure a shows the fractional systematic error on the fake rate due to the quark/gluon content of jet sample. In Figure b, the quadratic sum of the five components shown in figures 7.25 and $7.26 \mathrm{a}$ is shown.

\subsection{Application of Method: Cross-Check}

In order to cross-check the fake rate method for predicting the QCD background to the photon selection, the fake rate is applied to all jets in the JET_20, JET_50, JET_70 and JET_100 samples. The result of this prediction is compared to the number of photon candidates passing the photon selection in the same sample. This comparison is shown on a linear and log scale in figures 7.27 and 7.28 respectively. It is concluded that this method predicts the spectrum of photon candidates in jet triggered data in agreement to the actual spectrum found. 

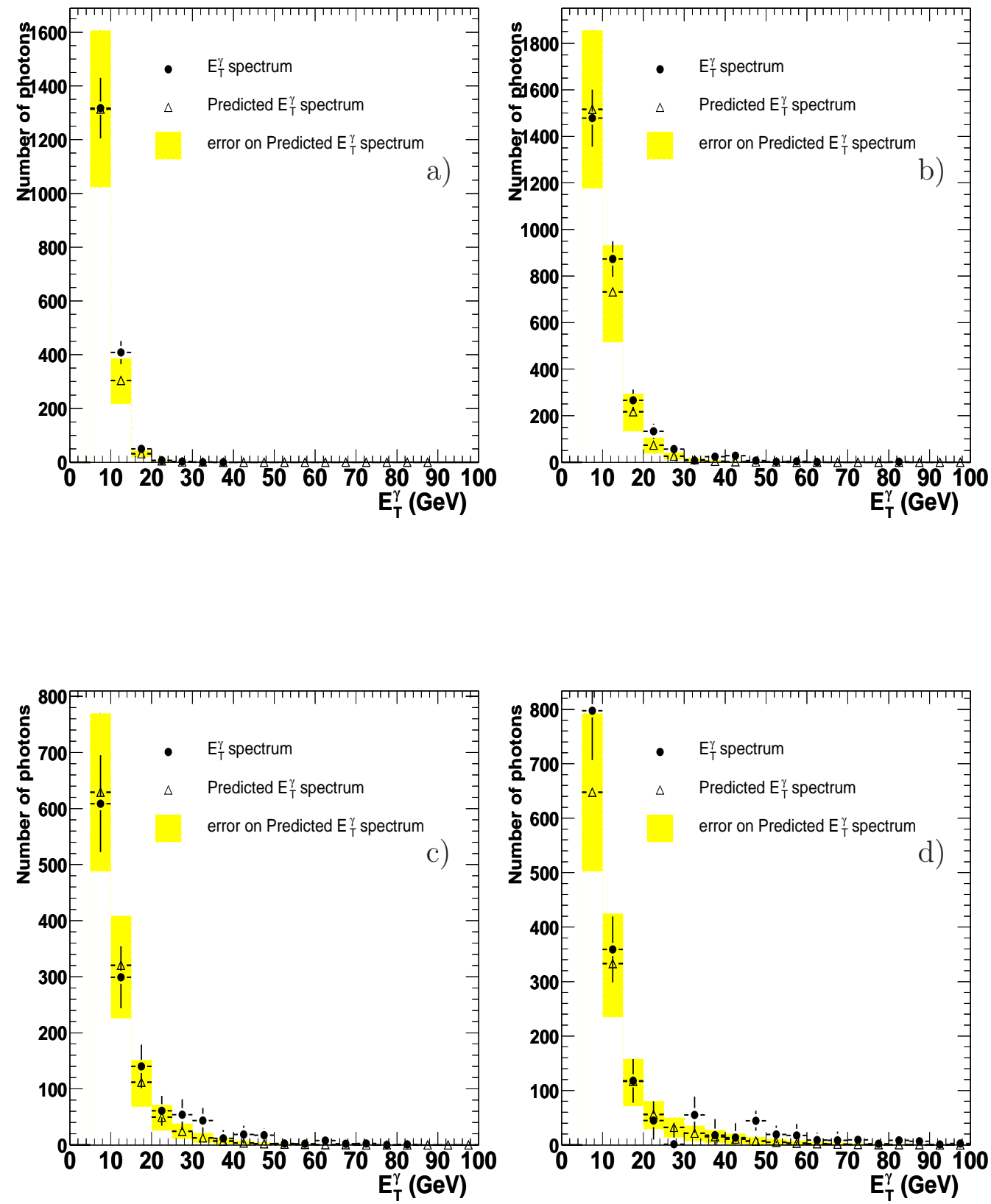

Figure 7.27: The observed fake photon spectrum compared to the fake rate prediction for the JET_20 (a), JET_50 (b), JET_70 (c) and JET_100 (d) datasets. 


\subsection{Predicted $Z^{0}+$ jet Background}

Table 7.2 shows the number of expected $Z^{0} \gamma$ candidates due to $Z^{0}+$ jet events where the jet fakes a photon. This prediction is achieved by applying the fake rate to jets produced in addition to a $Z^{0}$ candidate passing the selection given in chapter 4 .

\begin{tabular}{|l|c|}
\hline category & Number of expected events \\
\hline$Z_{C C}^{0} \gamma$ & $1.6 \pm 0.5$ \\
$Z_{C P}^{0} \gamma$ & $1.2 \pm 0.4$ \\
$Z_{\mu^{+} \mu^{-}}^{0}$ & $2.1 \pm 0.7$ \\
\hline$Z^{0} \gamma$ & $4.9 \pm 1.5$ \\
\hline
\end{tabular}

Table 7.2: The number of $Z^{0}+$ jet background events in the different $Z^{0} \gamma$ channels.

\subsection{Other Background Contributions}

There are three types of background events to this analysis:

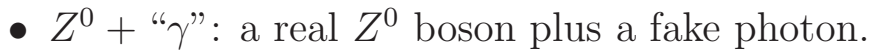

- " $Z^{0 "}+\gamma$ : a fake $Z^{0}$ boson plus a real photon.

- " $Z^{0 "}+$ " $\gamma$ ": a fake $Z^{0}$ boson plus a fake photon.

The $Z^{0}+$ " $\gamma$ " and " $Z$ " + " $\gamma$ " contributions are estimated using the fake rate described in section 7 . 

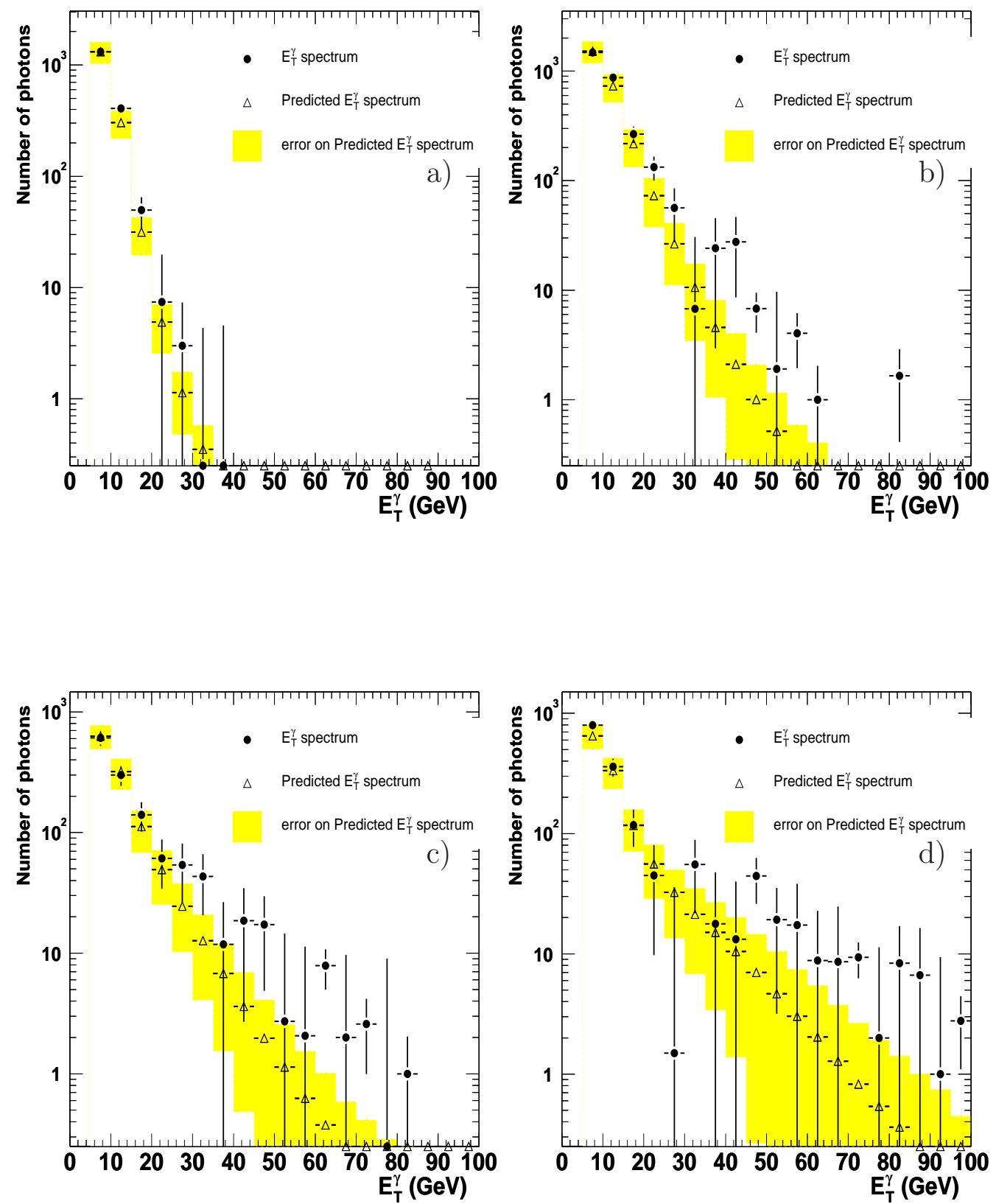

Figure 7.28: The observed fake photon spectrum compared to the fake rate prediction for the JET_20 (a), JET_50 (b), JET_70 (c) and JET_100 (d) datasets, on a log scale. 
For the estimate of the " $Z$ " $+\gamma$ background, the assumption is made that

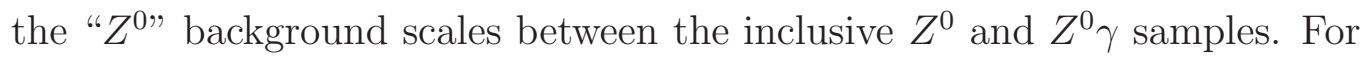
the inclusive $Z^{0}$ cross-section, the background contribution in section 5.3.2, was calculated by measuring the number of same-sign events $\left(N_{s s}(Z)\right)$. However, there are no same sign events in the $Z^{0} \gamma$ sample, i.e. $N_{s s}(Z \gamma)=0$. In order to get a background estimate, the electron identification cuts are loosened to the cuts given in table 7.4 and the assumption is made that the additional background obtained scales between the inclusive $Z^{0}$ sample and the $Z^{0} \gamma$ sample:

$$
\frac{N_{s s}(Z \gamma)}{N_{s s}(Z)}=\frac{N_{s s}^{\text {loose }}(Z \gamma)}{N_{s s}^{\text {loose }}(Z)}
$$

where $N_{s s}^{\text {loose }}(Z)$ and $N_{s s}^{\text {loose }}(Z \gamma)$ are the number of $Z^{0}$ and $Z^{0} \gamma$ same-sign events with the loose electron cuts.

In the $Z_{C C}^{0} \gamma$ channel, same-sign events can arise also from tridents. For this channel equation 7.24 is modified, by subtracting off this contribution, predicted with $Z^{0} \rightarrow e^{+} e^{-}$and ZGAMMA MC:

$$
N_{Z-B G}=\frac{\left(N_{s s}(Z)-N_{s s, m c}(Z)\right) \times\left(N_{s s}^{\text {loose }}(Z \gamma)-N_{s s, m c}^{\text {loose }}(Z \gamma)\right)}{\left(N_{s s}^{\text {loose }}(Z)-N_{s s, m c}^{\text {loose }}(Z)\right)}(7.25)
$$

With the loose $Z_{C C}^{0}$ selection given in table 7.4, 3 same-sign $Z_{C C}^{0} \gamma$ compared to 2698 same-sign $Z_{C C}^{0}$ events, i.e. $N_{s s}^{\text {loose }}(Z)=2698$ and $N_{s s}^{\text {loose }}(Z \gamma)=$ 3. Using the values given in in table 7.4, the background prediction for fake $Z_{C C}^{0}$ plus genuine photon events is $0.05 \pm 0.03$ (statistical error only).

There are no same-sign events for the inclusive $Z_{C M U P}^{0}$ and $Z_{C M X}^{0}$ channels, i.e. $N_{s s}(Z)=0$. It is therefore concluded that the background contribution in the $Z_{C M U P X}^{0} \gamma$ channel to be $<<1$. 
In order to estimate the background contribution to $Z_{C P}^{0} \gamma$ events, the fake rate method used in section 5.3.2 was employed with the additional requirement of a central photon passing the photon cuts of table 6.1, separated from both jets by $\Delta R_{\text {jet- } \gamma}>0.7$.

\begin{tabular}{|l|c|}
\hline category & Number of events \\
\hline$N_{s s}(Z)$ & 81 \\
$N_{s s, m c}(Z)$ & $28 \pm 2$ \\
$N_{s s}^{\text {loose }}(Z)$ & 2698 \\
$N_{s s, m c}^{\text {loose }}(Z)$ & $83 \pm 5$ \\
$N_{s s}^{\text {loose }}(Z \gamma)$ & 3 \\
$N_{s s, m c}^{\text {loose }}(Z \gamma)$ & $0.35 \pm 0.06$ \\
\hline
\end{tabular}

Table 7.3: The number of same sign events found for the background calculation of equation 7.25. The errors shown are statistical errors only in the MC prediction.

\begin{tabular}{|c|c|}
\hline Tight Central Electron & Loose Central Electron \\
\hline$E_{T}>20$ & $E_{T}>20$ \\
Iso $4<0.2$ & $|\eta|<1.1$ \\
All other cuts unchanged & no other cuts \\
\hline
\end{tabular}

Table 7.4: The loose $Z^{0} \rightarrow e^{+} e^{-}$selection used for estimating the fake $Z_{C C}^{0}$ plus $\gamma$ contribution.

The resulting values are $0.05 \pm 0.03$ for $\mathrm{CC}$ and $0.01 \pm 0.01$ for $\mathrm{CP} Z^{0} \gamma \rightarrow$ eer events. For the $Z^{0} \gamma \rightarrow \mu \mu \gamma$ channel, no same-sign event is found, even with loose cuts. 
It is concluded that this source of background is negligible compared to the fake rate and associated systematic errors summarised in section 7.7.6. 


\section{Chapter 8}

\section{Systematic Errors}

In this section, the systematic errors and their impact on the $Z^{0} \gamma$ production cross-section are summarised. The errors are categorised as experimental, theoretical and luminosity. The sources of systematic errors are as follows:

- An $E_{T}^{\gamma}$ dependent uncertainty in the photon fake rate is assigned in section 7.7.6. This leads to a $2 \%$ error on the $Z^{0} \gamma$ cross-section measurement, in both the electron and muon channels.

- A $1 \%$ error is attributed to the muon and central electron ID and reconstruction efficiencies and a $2.5 \%$ error for the plug electron ID efficiency. Details are given in sections 4.2 .5 and 4.3 .5 for the electron and muon channels respectively. These contribute to a $2 \%$ error in the $Z^{0} \gamma$ cross-section.

- In the muon channel there is a $0.06 \%$ contribution to the total systematic error on the cross-section measurement by the inefficiency of the cosmic ray tagger, explained in section 5.3.1. 
- There is a $0.4 \%$ uncertainty due to the efficiency of the $\left|z_{0}\right|<60 \mathrm{~cm}$ cut described in section 4.1.

- The photon ID efficiency is discussed in section 6.2 , with the final efficiencies and associated systematic error of $2.3 \%$ given in section 6.2 .4 .

- The uncertainty on the conversion rate of $1.5 \%$ is given in section 6.2.3.

- The uncertainties on the central electron, CMUP muon and CMX muon triggers, are all 1\% (see section 4.3.1).

- There is an uncertainty on the electron energy and muon momentum scales of $1 \%[63,62]$. This results in a $2.5 \%$ error on the $Z^{0} \gamma$ crosssection.

- The electron energy scale precision has been measured to be $1 \%$ in the region of the $Z^{0}$ mass. Figure 8.1 shows that the data and $\mathrm{MC}$ to agree to within $1 \%$ for $E_{T}<15 \mathrm{GeV}$ from electrons from the decay of a $J / \Psi$. To take into account possible non-linearities with this error, as a function of $E_{T}$, the error is increased to $3 \%$. The resulting error on the $Z^{0} \gamma$ cross-section is $2 \%$.

- The systematic error on the acceptance is determined using ZGAMMA MC. The QCD corrections in the NLO theory can involve the emission of an additional jet. This can result in a slight change in the kinematics of the event, thus altering the acceptance of the event between the LO and NLO predictions. This effect was quantified by studying the acceptance for the analysis cuts, table 8.1, for the LO and NLO calcu- 


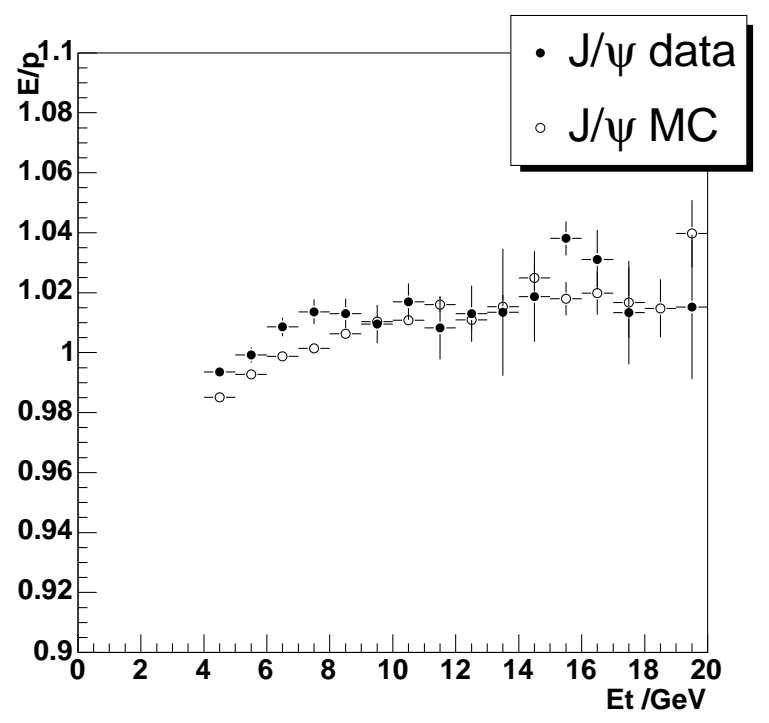

Figure 8.1: The two $E / P$ distributions of electrons from the decay of a $J / \Psi$, in data and MC, agree to within $1 \%$. 
lations. For $\left|\eta_{l e p}\right|<1.0$ the acceptance change was $2.5 \%$, whereas if the $\eta$ range is increased to $\left|\eta_{l e p}\right|<2.6$ the acceptance change decreased to $1.0 \%$. Therefore the uncertainty is taken to be $2.5 \%(1.0 \%)$ in the $\mu$ (e) channel.

\begin{tabular}{|c|c|}
\hline$P_{T}^{\gamma}$ & $>7 \mathrm{GeV}$ \\
$\left|\eta_{\gamma}\right|$ & $<1.0$ \\
$P_{T}^{l e p}$ & $>25$ \\
$\left|\eta_{l e p}\right|$ & varied \\
$P_{T}^{j e t}$ & $>0$ \\
$\left|\eta_{j e t}\right|$ & $<10.0$ \\
$\Delta R_{l \gamma}$ & $>0.7$ \\
$M_{I N V}(l l)$ & $40 \mathrm{GeV} / \mathrm{c}^{2}$ \\
\hline
\end{tabular}

Table 8.1: $Z^{0} \gamma$ selection for acceptance systematic

In order to calculate the total systematic uncertainty on the combined cross-section measurement, the correlations of the errors between the different channels needs to be considered. These correlations are illustrated in table 8.2. The errors are classed in different groups. Errors which only contribute to a single channel, $\left(Z_{C C}^{0} \gamma, Z_{C P}^{0} \gamma, Z_{C M U P}^{0} \gamma\right.$ and $\left.Z_{C M X}^{0} \gamma\right)$, are added in quadrature to give the combined errors $\delta_{s y s}^{c c}, \delta_{s y s}^{c p}, \delta_{\text {sys }}^{c m u p}$ and $\delta_{\text {sys }}^{c m x}$. Any errors which contribute to both electron(muon) channels are added in quadrature to give the value for term $\delta_{\text {sys }}^{c c c p}\left(\delta_{\text {sys }}^{c m u p x}\right)$. Finally all errors which contribute to all channels are added in quadrature to give $\delta_{\text {sys }}^{\text {all }}$.

The systematic error for the combined $Z^{0} \gamma$ channel is then given by: 


\begin{tabular}{|c|c|c|c|c|c|c|}
\hline Source & $\%$ & $\%$ effect on $\sigma$ & $Z_{C C}^{0} \gamma$ & $Z_{C P}^{0} \gamma$ & $Z_{C M U P}^{0} \gamma$ & $Z_{C M X}^{0} \gamma$ \\
\hline Jet Fake & $20-200$ & $\sim 2$ & $\mathrm{x}$ & $\mathrm{x}$ & $\mathrm{x}$ & $\mathrm{x}$ \\
\hline Acceptance (e) & 1 & 1 & $\mathrm{x}$ & $\mathrm{x}$ & - & - \\
\hline Acceptance $(\mu)$ & 2.5 & 2.5 & - & - & $\mathrm{x}$ & $\mathrm{x}$ \\
\hline $\mathrm{Z} 0$ cut eff & 0.4 & 0.4 & $\mathrm{x}$ & $\mathrm{x}$ & $\mathrm{x}$ & $\mathrm{x}$ \\
\hline Photon cut eff & 2.3 & 2.3 & $\mathrm{x}$ & $\mathrm{x}$ & $\mathrm{x}$ & $\mathrm{x}$ \\
\hline energy scale $(\gamma)$ & 3.0 & 2.0 & $\mathrm{x}$ & $\mathrm{x}$ & $\mathrm{x}$ & $\mathrm{x}$ \\
\hline conv. rate uncer & 1.5 & 1.5 & $\mathrm{x}$ & $\mathrm{x}$ & $\mathrm{x}$ & $\mathrm{x}$ \\
\hline Central e ID & 1.0 & 2.0 & $\mathrm{x}$ & $1.0 \%$ & - & - \\
\hline central trig & 1.0 & 1.0 & $\mathrm{x}$ & $\mathrm{x}$ & - & - \\
\hline e Energy Scale & 1 & 2.5 & $\mathrm{x}$ & $\mathrm{x}$ & - & - \\
\hline plug eID & 2.5 & 2.5 & - & $\mathrm{x}$ & - & - \\
\hline plug track eff & 1.5 & 1.5 & - & $\mathrm{x}$ & - & - \\
\hline cosmic & 0.06 & 0.06 & - & - & $\mathrm{x}$ & $\mathrm{x}$ \\
\hline COT track rec & 0.4 & 0.8 & $\mathrm{x}$ & $0.4 \%$ & $\mathrm{x}$ & $\mathrm{x}$ \\
\hline$P_{T}$ scale $(\mu)$ & 1 & 2.5 & - & - & $\mathrm{x}$ & $\mathrm{x}$ \\
\hline CMUP ID & 0.7 & 0.7 & - & - & $\mathrm{x}$ & - \\
\hline CMUP rec & 0.6 & 0.6 & - & - & $\mathrm{x}$ & - \\
\hline CMUP trig & 0.8 & 0.8 & - & - & $\mathrm{x}$ & - \\
\hline CMX ID & 0.8 & 0.8 & - & - & - & $\mathrm{x}$ \\
\hline CMX rec & 0.3 & 0.3 & - & - & - & $\mathrm{x}$ \\
\hline CMX trig & 0.6 & 0.6 & - & - & - & $\mathrm{x}$ \\
\hline loose muon ID & 0.6 & 0.6 & - & - & $\mathrm{x}$ & $\mathrm{x}$ \\
\hline
\end{tabular}

Table 8.2: A summary of all systematic errors considered for the $Z^{0} \gamma$ crosssection and their correlations between the $Z_{C C}^{0}, Z_{C P}^{0}, Z_{C M U P}^{0}$ and $Z_{C M X}^{0}$ channels. An " $\mathrm{x}$ " indicates the uncertainty is applied to that channel and thus correlated to all the other channels with the same systematic. If a different value is applied to the $Z_{C P}^{0} \gamma$, due to only one lepton in the central region of the detector, its value is indicated. 


$$
\begin{aligned}
\left(\delta_{\text {sys }}\left(N_{Z \gamma}\right)\right)^{2}= & \left(N_{Z \gamma}^{c c} \delta_{\text {sys }}^{c c}\right)^{2}+\left(N_{Z \gamma}^{c p} \delta_{\text {sys }}^{c p}\right)^{2}+\left(N_{Z \gamma}^{c c c p} \delta_{\text {sys }}^{c c p p}\right)^{2} \\
& +\left(N_{Z \gamma}^{c m u p} \delta_{\text {sys }}^{c m u p}\right)^{2}+\left(N_{Z \gamma}^{c m x} \delta_{\text {sys }}^{c m x}\right)^{2}+\left(N_{Z \gamma}^{c m u p x} \delta_{\text {sys }}^{c m u p x}\right)^{2} \\
& +\left(N_{Z \gamma}^{\text {all }} \delta_{\text {sys }}^{\text {all }}\right)^{2}
\end{aligned}
$$




\section{Chapter 9}

\section{Results}

Using the event selection detailed in sections 4, and selecting dilepton pairs with $40<m(l, l)<130 \mathrm{GeV} / \mathrm{c}^{2}, 71 Z^{0} \gamma$ events are observed compared to a Standard Model expectation of signal plus background of $69.6 \pm 3.0($ sys $) \pm$ $3.9($ lum $) \pm 4.6(t h)$. The errors quoted are the systematic experimental uncertainties( discussed in section 8), the $6 \%$ luminosity error (see section 3.3.6) and the $7 \%$ error on the theoretical uncertainty from section 2.2.3. The breakdown of the candidate events for the different analysis channels is given in Table 9.1. A summary table for the total number of candidates observed is presented in Table 9.2.

Comparisons of how the kinematic distributions of the events compare to prediction are made in section 9.1. Details on the cross-section calculation, for $Z^{0} \gamma$ production in the kinematic range $\Delta R>0.7$ and $E_{t}^{\gamma}>7$, are presented in section 9.2 and the results given. 


\begin{tabular}{|l|r|r|r|}
\hline & \multicolumn{3}{|c|}{ Number of Events } \\
\hline & \multicolumn{2}{|c|}{$Z^{0} \gamma \rightarrow e^{+} e^{-} \gamma$} & $Z^{0} \gamma \rightarrow \mu^{+} \mu^{-} \gamma$ \\
& $\mathrm{CC}$ & $\mathrm{CP}$ & \\
\hline$Z^{0}+\gamma$ & $19.7 \pm 0.8$ & $11.4 \pm 0.6$ & $33.6 \pm 1.5$ \\
$Z^{0}+j e t$ & $1.6 \pm 0.5$ & $1.2 \pm 0.37$ & $2.1 \pm 0.7$ \\
total SM $+\mathrm{BG}$ & $21.3 \pm 0.9$ & $12.6 \pm 0.7$ & $35.7 \pm 1.7$ \\
\hline Data & 24 & 12 & 35 \\
\hline
\end{tabular}

Table 9.1: Numbers of events in data and those expected for $Z^{0} \gamma \rightarrow e^{+} e^{-} \gamma$ and $Z^{0} \gamma \rightarrow \mu^{+} \mu^{-} \gamma$

\begin{tabular}{|l|r|}
\hline & Number of Events \\
\hline$Z^{0}+\gamma$ & $64.6 \pm 2.6$ \\
$Z^{0}+$ jet & $4.9 \pm 1.5$ \\
total SM + BG & $69.6 \pm 3.0$ \\
\hline Data & 71 \\
\hline
\end{tabular}

Table 9.2: Numbers of events in data and those expected for $Z^{0} \gamma \rightarrow l^{+} l^{-} \gamma$, after combining the separate channels. 


\subsection{Kinematic Distributions}

In order to test whether the observed events are in agreement with the expected results, it is useful to compare various kinematic distributions, in addition to comparing the total number of events observed and predicted. By comparing these distributions in the individual analysis channels and the combined data will give greater sensitivity to any anomalous behaviour. Figures 9.1 and 9.2 show the $E_{T}^{\gamma}$ distribution on linear and $\log$ scales. The $\Delta R_{l \gamma}$ distribution is shown in figure 9.3 and the dilepton and three-body invariant mass distributions are shown in figures 9.4 and 9.5 respectively. Figures 9.1 to 9.5 show good agreement between the observed data and the SM prediction. A plot of the three-body invariant mass distribution versus the dilepton invariant mass is shown in figure 9.6.

\section{2 $\quad Z^{0} \gamma$ Cross Sections}

The cross section is given by

$$
\sigma=\frac{N_{\text {Data }}-N_{B G}}{\mathcal{L} \cdot A \cdot \epsilon_{M C} \cdot \epsilon^{\text {corr }}}
$$

where $N_{\text {Data }}$ is the number of observed events, $N_{B G}$ the number of background events, $A$ the acceptance for this kinematic range (see section 6.3), $\epsilon_{M C}$ the efficiency in $\mathrm{MC}$ (section 6.3) and $\epsilon_{\text {corr }}$ the correction to reproduce the data efficiency (sections 4.2.5, 4.3.5 and 6.2.4).

With $N_{Z^{0} \gamma M C}^{e x p}=\sigma_{M C} A \mathcal{L} \epsilon_{M C} \epsilon^{c o r r}$ being the expected number of $Z^{0} \gamma$ events this simplifies to

$$
\sigma=\frac{N_{D a t a}-N_{B G}}{N_{Z^{0} \gamma M C}^{e x p}} \times \sigma_{E_{t}>5 G e V, \Delta R>0.2}^{M C}
$$



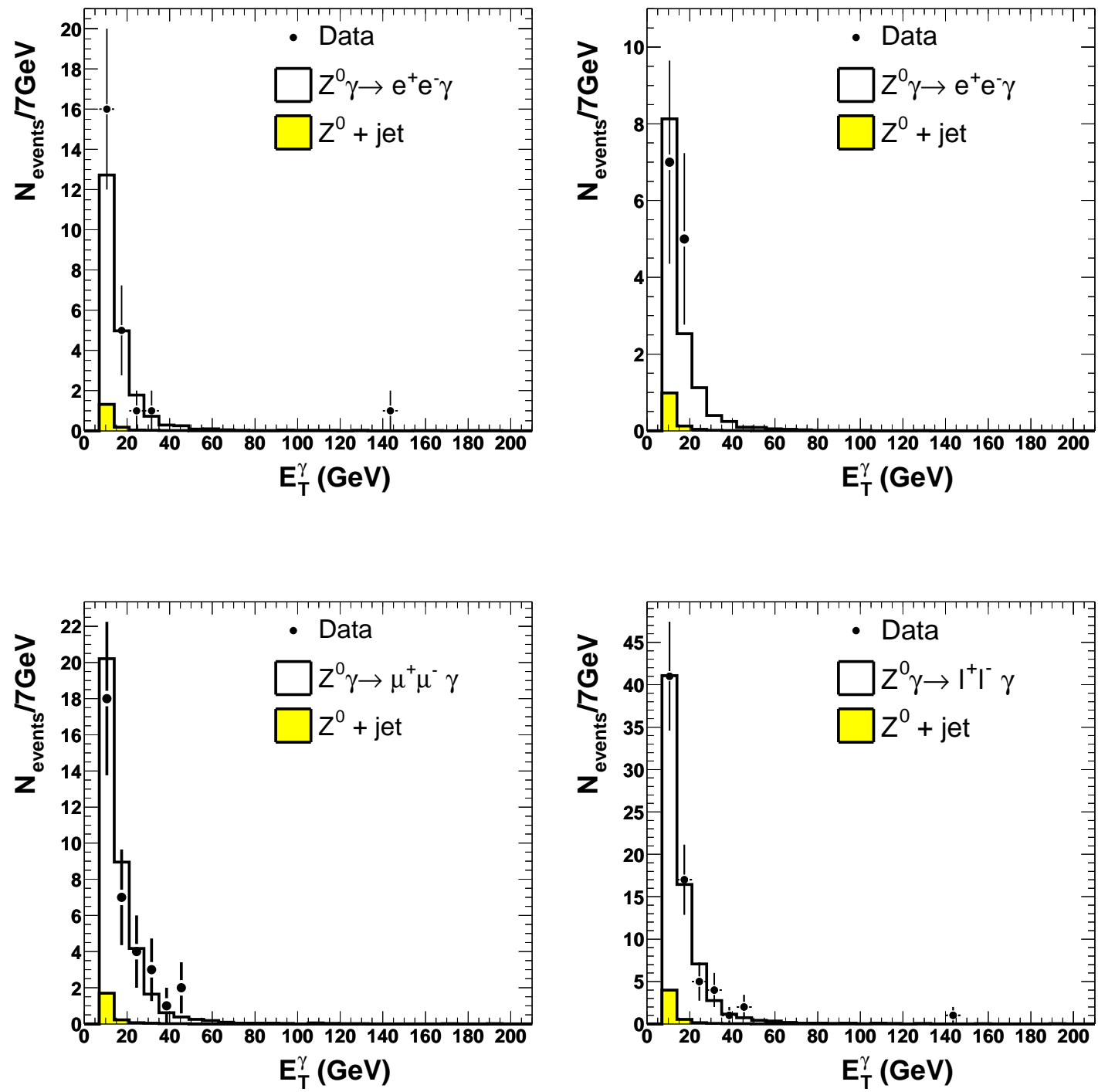

Figure 9.1: Photon $E_{T}$ spectrum for $\mathrm{CC}$ (top-left), $\mathrm{CP}$ (top right), $\mu \mu$ (bottom left) and combined $Z^{0} \gamma$ (bottom right) channels 

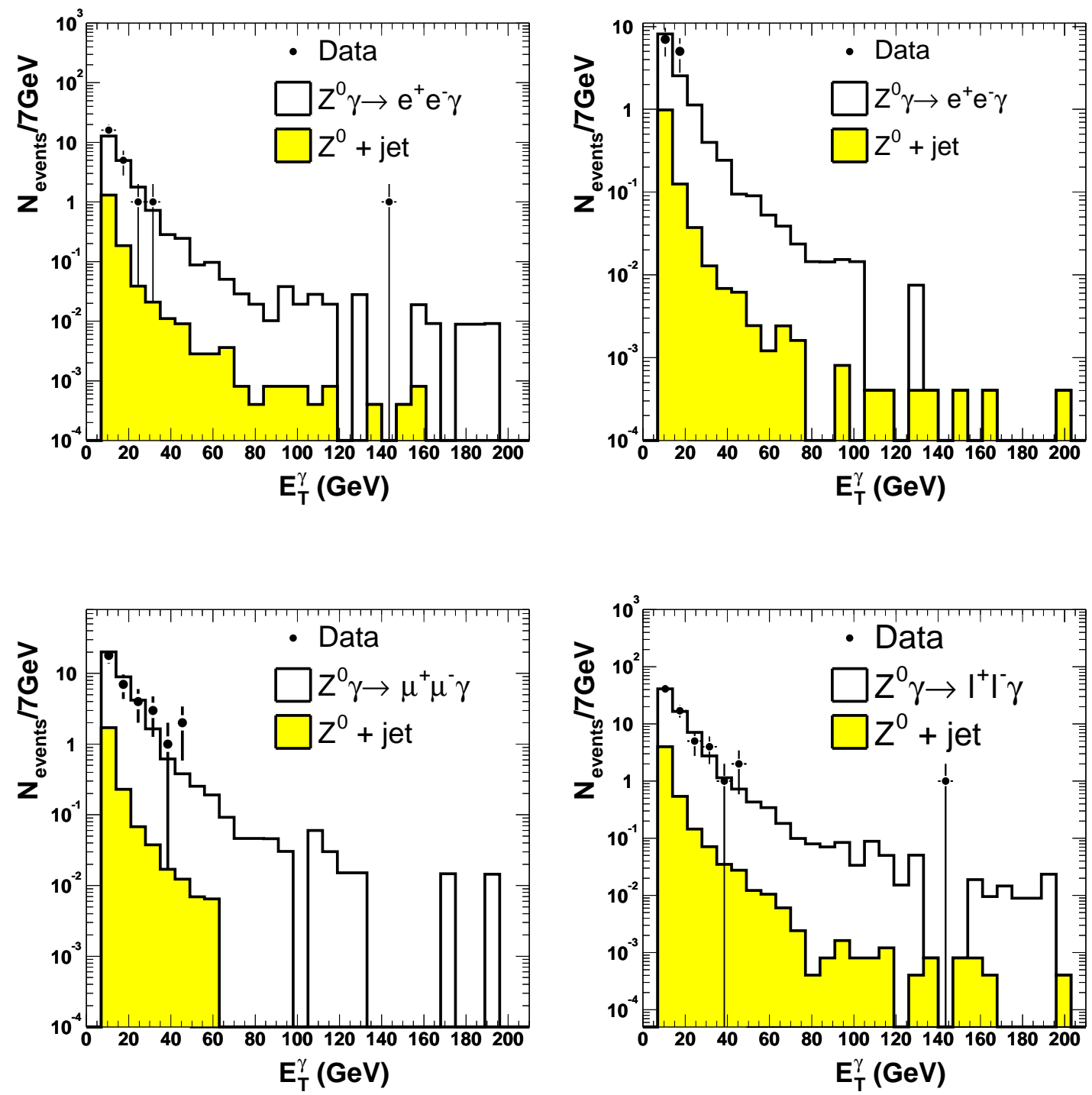

Figure 9.2: Photon $E_{T}$ spectrum, on a log scale, for CC(top-left), CP(top right), $\mu \mu$ (bottom left) and combined $Z^{0} \gamma$ (bottom right) channels 

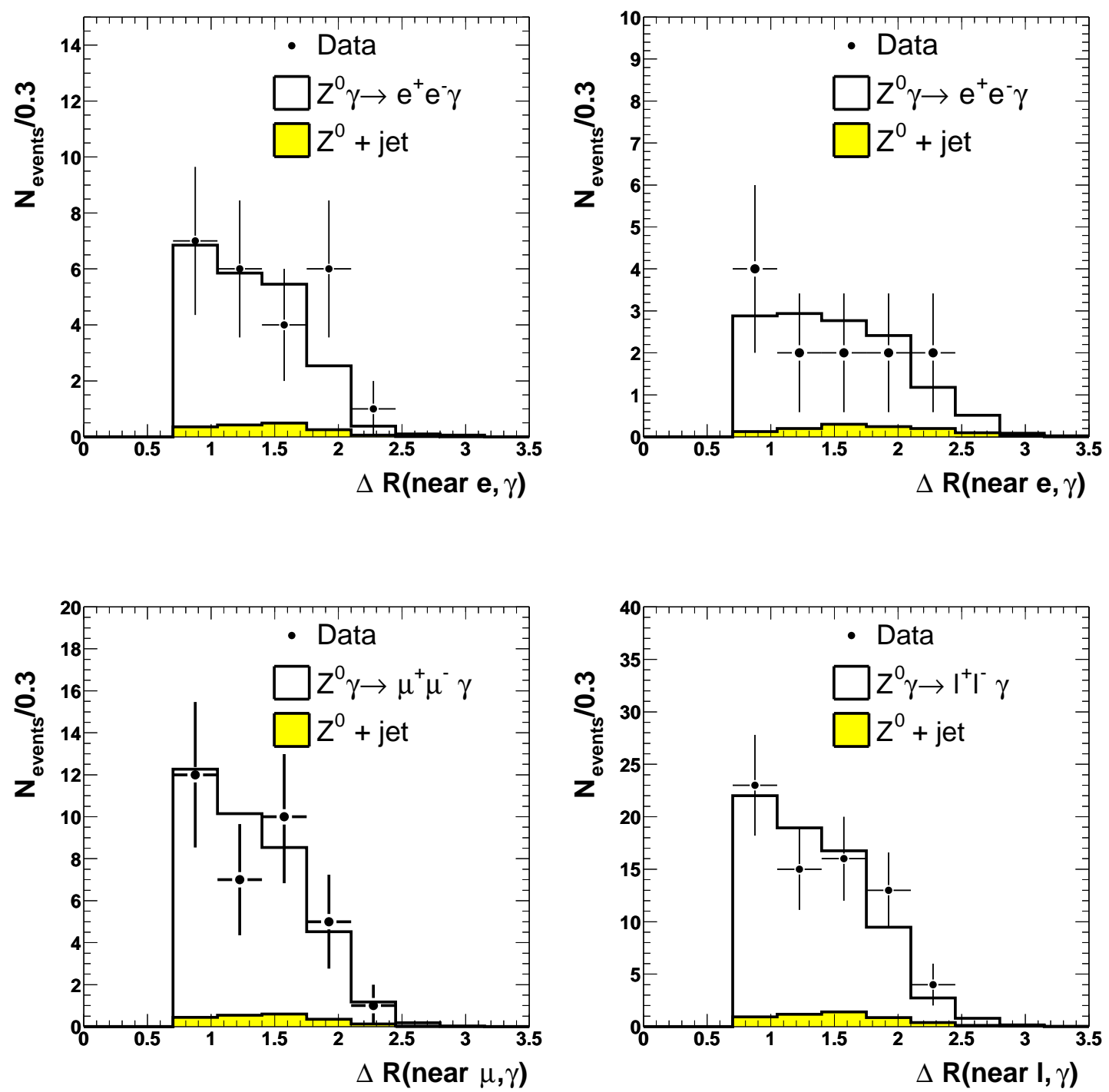

Figure 9.3: $\Delta R_{l \gamma}$ distribution between the photon and closest lepton for $\mathrm{CC}$ (top-left), $\mathrm{CP}$ (top right), $\mu \mu$ (bottom left) and combined $Z^{0} \gamma$ (bottom right) channels 

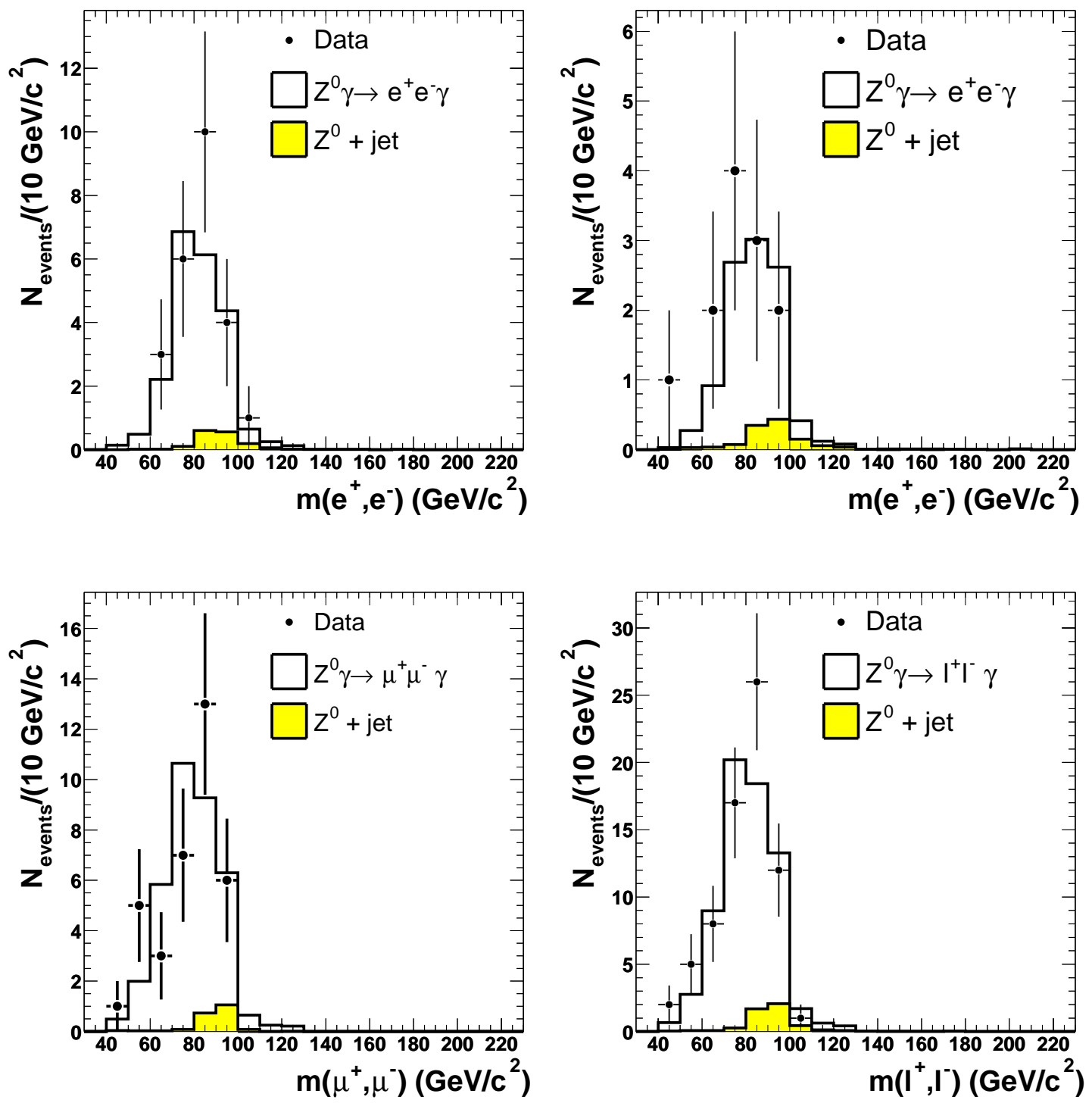

Figure 9.4: The dilepton invariant mass distribution for CC(top-left), CP(top right), $\mu \mu$ (bottom left) and combined $Z^{0} \gamma$ (bottom right) channels 

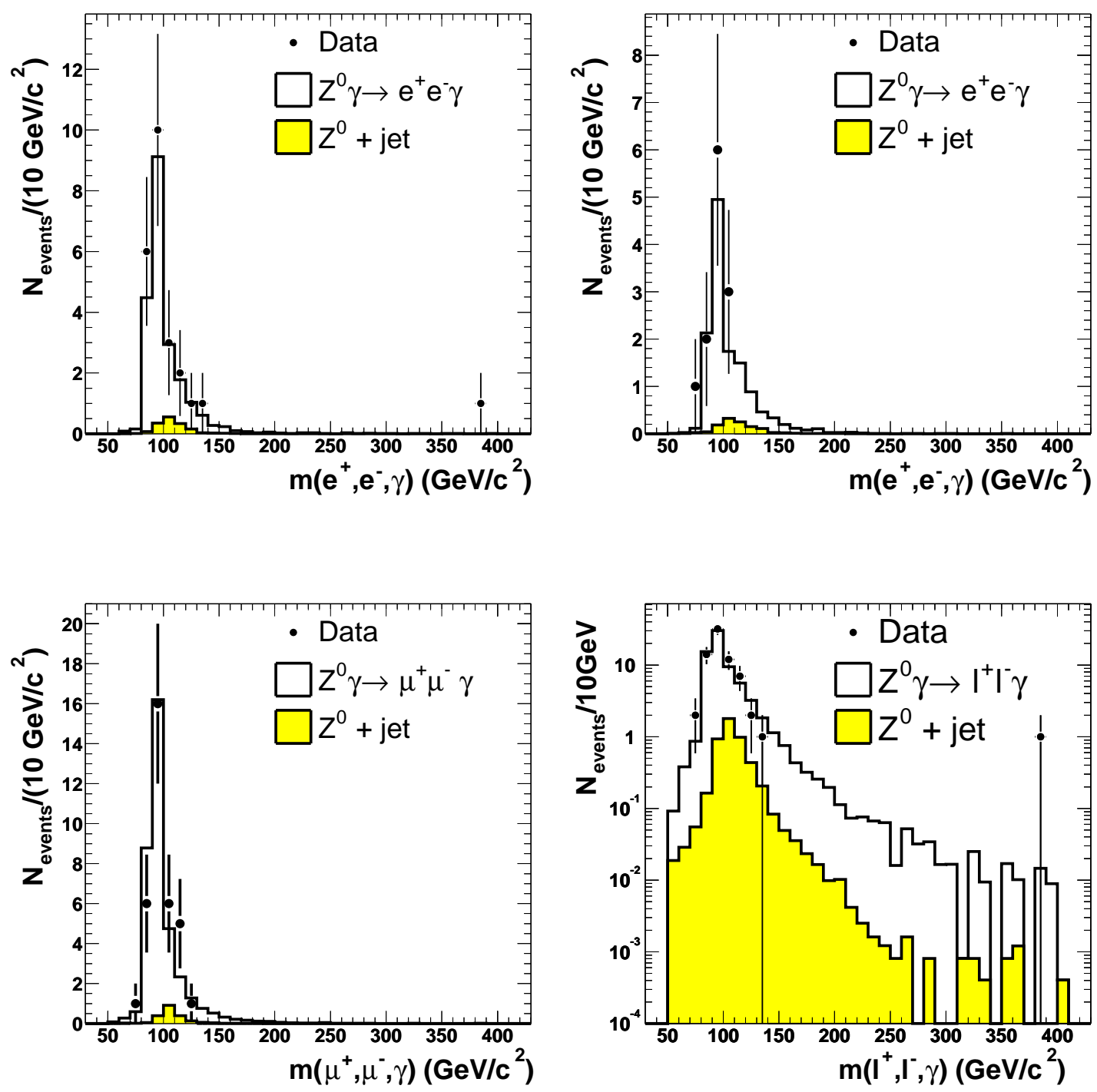

Figure 9.5: The three-body invariant mass distribution for CC(top-left), $\mathrm{CP}$ (top right), $\mu \mu$ (bottom left) and combined $Z^{0} \gamma$ (bottom right) channels 


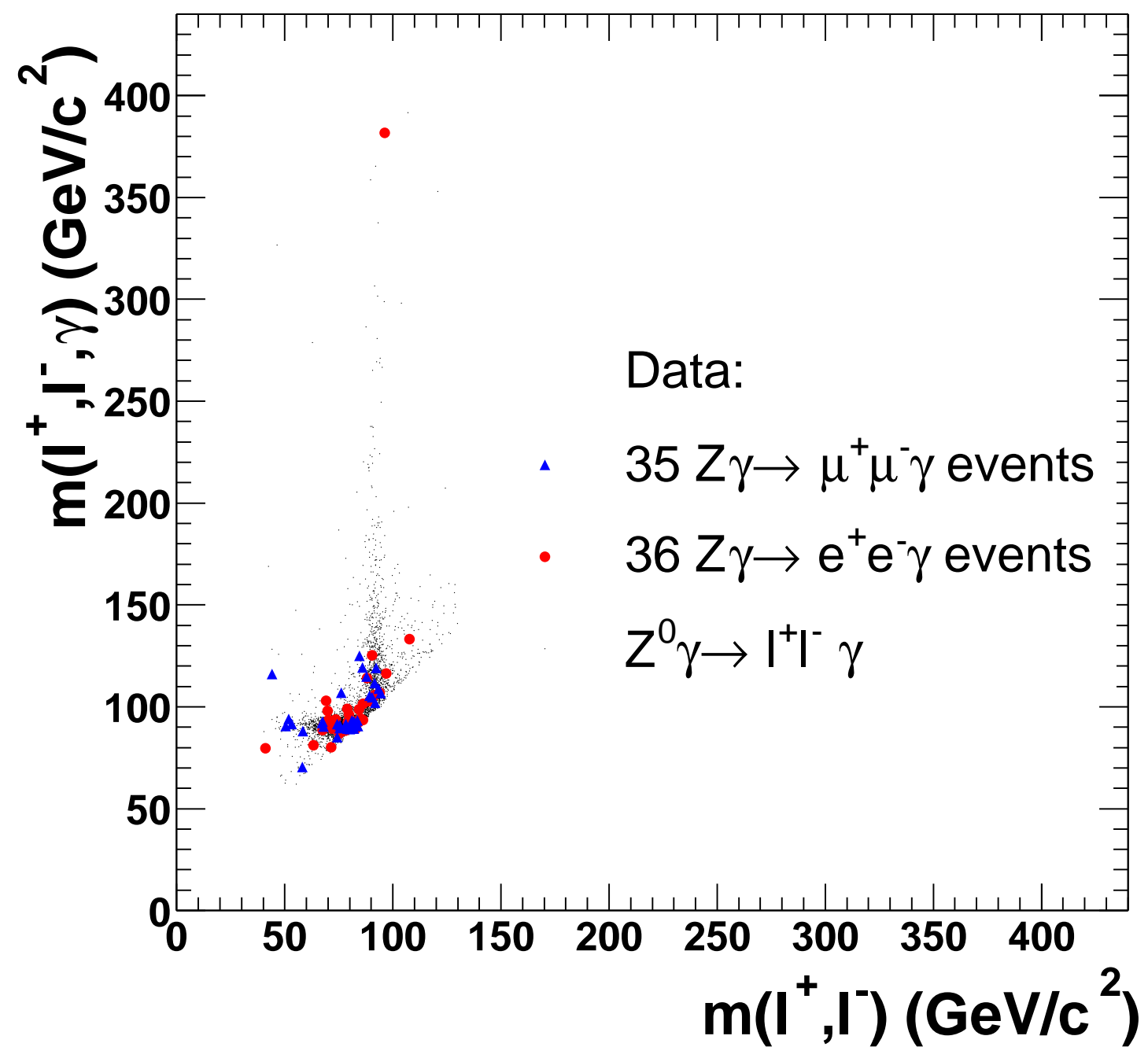

Figure 9.6: Invariant mass of the two leptons and the photon versus the dilepton invariant mass for $Z^{0} \gamma$ candidates in the electron (circle) and muon (triangle) channels. The black dots are the expectations from the $Z^{0} \gamma \mathrm{MC}$. 
for the kinematic range of the ZGAMMA MC. In order to correct back to the kinematic range of the measurement, equation 9.1 is scaled by the ratio of the theoretical cross section in the two kinematic regions (see section 2.2.1):

$$
\sigma_{E_{t}>7 G e V, \Delta R>0.7}^{M C} / \sigma_{E_{t}>5 G e V, \Delta R>0.2}^{M C}=4.47 / 11.55=0.387
$$

Thus the cross section in the kinematic range $\Delta R_{l \gamma}>0.7$ and $E_{t}^{\gamma}>7$ $\mathrm{GeV}$ is given simply by

$$
\begin{aligned}
\sigma & =\frac{N_{\text {Data }}-N_{B G}}{N_{Z^{0} \gamma M C}^{e x p}} \times \sigma_{\text {default }}^{M C} \times \sigma_{E_{t}>7 G e V, \Delta R>0.7}^{M C} / \sigma_{\text {default }}^{M C} \\
& =\frac{N_{\text {Data }}-N_{B G}}{N_{Z^{0} \gamma M C}^{e x p}} \times \sigma_{E_{t}>7 G e V, \Delta R>0.7}^{M C}
\end{aligned}
$$

The cross sections results for $Z^{0} \gamma$ are:

- $Z^{0} \gamma \rightarrow e^{+} e^{-} \gamma(\mathrm{CC}): \sigma \times B R=5.1 \pm 1.0$ (stat) \pm 0.2 (sys) \pm 0.3 (lumi) pb

- $Z^{0} \gamma \rightarrow e^{+} e^{-} \gamma(\mathrm{CP}): \sigma \times B R=4.3 \pm 1.2$ (stat) \pm 0.3 (sys) \pm 0.3 (lumi) pb

- $Z^{0} \gamma \rightarrow e^{+} e^{-}: \sigma \times B R=4.8 \pm 0.8$ (stat) \pm 0.3 (sys) \pm 0.3 (lumi) pb

- $Z^{0} \gamma \rightarrow \mu^{+} \mu^{-} \gamma: \sigma \times B R=4.4 \pm 0.8$ (stat) \pm 0.2 (sys) \pm 0.3 (lumi) pb

resulting in a combined cross section of $\sigma \times B R=4.6 \pm 0.5$ (stat) \pm 0.2 (sys) \pm 0.3(lumi) pb, compared to the theoretical prediction of $4.5 \pm 0.3$ (th.) pb.

\subsection{Comparison to $W^{ \pm} \gamma$}

Parallel to the $Z^{0} \gamma$ analysis at CDF, the production cross-section of $W^{ \pm} \gamma$ events has been measured in the kinematic range $\Delta R_{l \gamma}>0.7$ and $E_{t}^{\gamma}>7[117$, 118]. $W^{ \pm} \rightarrow l^{ \pm} \nu$ events are selected by selecting a tight central electron, a 


\begin{tabular}{|l|l|l|}
\hline Central Electron & $E_{T}>25$ & $\mathbb{E}_{T}>25$ \\
Plug Electron & $E_{T}>25$ & $\mathbb{E}_{T}>25$ \\
CMUP Muon & $P_{T}>20$ & $\mathbb{E}_{T}>20$ \\
CMX Muon & $P_{T}>20$ & $\mathbb{E}_{T}>20$ \\
\hline
\end{tabular}

Table 9.3: Lepton $E_{T}$ and $\mathbb{E}_{T}$ cuts used for the different $W^{ \pm} \gamma$ channels.

plug electron, CMUP or a CMX muon using the lepton selection given in chapter 4 plus a missing transverse energy signature. A summary of the different categories of $W^{ \pm} \gamma$ events is given in table 9.3.

Any neutrinos produced at CDF, will escape the detector without interacting. From conservation of momentum, there will be an observable energy imbalance or missing energy in the event, associated with energy carried away by the neutrino. The missing transverse energy of the event is calculated by summing the energy deposits in all calorimeter towers within the region $|\eta|<3.6$.

The cross-section results for $W^{ \pm} \gamma$ are:

- Central Electron: $\sigma \times B R=20.1 \pm 2.6$ (stat) \pm 2.7 (sys) \pm 1.2 (lumi) pb

- Plug Electron: $\sigma \times B R=18.4 \pm 3.8$ (stat) \pm 3.4 (sys) \pm 1.1 (lumi) pb

- CMUP Muon: $\sigma \times B R=18.8 \pm 2.9$ (stat) $\pm 1.8($ sys $) \pm 1.1$ (lumi) $\mathrm{pb}$

- CMX Muon: $\sigma \times B R=11.5 \pm 3.6$ (stat) \pm 1.8 (sys) \pm 0.7 (lumi) pb resulting in a combined cross section of $\sigma \times B R=18.1 \pm 3.1 \mathrm{pb}$, compared to the theoretical prediction of $19.3 \pm 1.4($ th. $)$ pb. 


\section{Chapter 10}

\section{Summary and Outlook}

The $Z^{0} \gamma$ production cross-section in the $Z^{0} \rightarrow e^{+} e^{-}$and $Z^{0} \rightarrow \mu^{+} \mu^{-}$decay channels has been measured at $\sqrt{s}=1.96 \mathrm{TeV}$. In a total of $202 \mathrm{pb}^{-1}$ integrated luminosity, 71 candidate events have been observed compared to a measured NLO SM prediction of $69.6 \pm 3.0$. The combined cross-section are measured to be $\sigma \times B R=4.6 \pm 0.5$ (stat) \pm 0.2 (sys) \pm 0.3 (lumi) pb, compared to the theoretical prediction of $4.5 \pm 0.3$ (th.) pb.

The Tevatron will continue to collect data until until 2008-2009, when the Large Hadron Collider becomes competitive. At that point both CDF and D0 will have collected around $5-8 \mathrm{fb}^{-1}$ of data. From this larger dataset, competitive limits on the $Z Z \gamma, Z \gamma \gamma$ and $W W \gamma$ anomalous trilinear gauge couplings can be placed. 


\section{Appendix A}

\section{Appendix}

\section{A.1 Lepton identification efficiencies}

\begin{tabular}{|c|c|c|}
\hline \hline Variable & Data & $Z^{0} \rightarrow e^{+} e^{-} \mathrm{MC}$ \\
\hline$E_{\text {had }} / E_{\text {em }}$ & $99.4 \pm 0.5$ & $98.97 \pm 0.05$ \\
$\mathrm{E} / \mathrm{P}$ & $93.3 \pm 0.4$ & $94.22 \pm 0.11$ \\
$I s o 4 / E_{T}$ & $98.0 \pm 0.4$ & $98.22 \pm 0.06$ \\
$L_{\text {shr }}$ & $99.4 \pm 0.5$ & $97.49 \pm 0.07$ \\
$q \cdot \Delta X$ & $99.9 \pm 0.5$ & $99.48 \pm 0.03$ \\
$|\Delta Z|$ & $99.9 \pm 0.5$ & $99.93 \pm 0.01$ \\
$\chi_{\text {strip }}^{2}$ & $96.9 \pm 0.4$ & $98.60 \pm 0.05$ \\
$N_{\text {Ax }} \mathrm{SL}$ & $99.7 \pm 0.5$ & $99.86 \pm 0.02$ \\
$N_{S t} \mathrm{SL}$ & $97.8 \pm 0.4$ & $99.51 \pm 0.03$ \\
\hline Total $\epsilon_{\text {tight }}$ & $82.5 \pm 0.5$ & $85.56 \pm 0.16$ \\
Total $\epsilon_{\text {tight }}$ & $94.1 \pm 0.3$ & $96.24 \pm 0.08$ \\
\hline
\end{tabular}

Table A.1: N-1 efficiencies for the central electron identification variables. [61] 


\begin{tabular}{|c|c|c|}
\hline \hline Variable & Data & $Z^{0} \rightarrow e^{+} e^{-} \mathrm{MC}$ \\
\hline PEM 3by3 $\chi^{2}$ & $91.2 \pm 0.8$ & $94.9 \pm 0.1$ \\
$I$ so $4 / E_{T}$ & $98.6 \pm 0.3$ & $98.2 \pm 0.1$ \\
$E_{\text {had }} / E_{\text {em }}$ & $99.0 \pm 0.3$ & $98.7 \pm 0.2$ \\
$\mathrm{PES} \mathrm{U} 5 \times 9$ & $99.3 \pm 0.3$ & $99.6 \pm 0.1$ \\
$\mathrm{PES} \mathrm{V} 5 \times 9$ & $99.2 \pm 0.3$ & $99.7 \pm 0.1$ \\
Total $\epsilon_{\text {plug }}$ & $85.9 \pm 1.0$ & $91.2 \pm 0.2$ \\
\hline
\end{tabular}

Table A.2: N-1 efficiencies for the plug electron identification variables. (excluding PHOENIX track requirement [64].

\begin{tabular}{|c|c|c|}
\hline \hline & CMUP \\
Variable & Data & $Z^{0} \rightarrow \mu^{+} \mu^{-} \mathrm{MC}$ \\
\hline$d_{0}$ & $99.6 \pm 0.1$ & $99.86 \pm 0.02$ \\
$E_{\text {em }}$ & $96.8 \pm 0.3$ & $95.96 \pm 0.13$ \\
$E_{\text {had }}$ & $98.2 \pm 0.2$ & $97.63 \pm 0.10$ \\
$N_{A x} \mathrm{SL}, N_{S t}$ SL & $97.6 \pm 0.3$ & $99.52 \pm 0.05$ \\
\hline iso & $98.0 \pm 0.3$ & $97.85 \pm 0.10$ \\
\hline CMUP stub & $91.3 \pm 0.56$ & $96.4 \pm 0.12$ \\
$\left|\Delta x_{C M U}\right|$ & $95.4 \pm 0.4$ & $99.85 \pm 0.03$ \\
$\left|\Delta x_{C M P}\right|$ & $98.0 \pm 0.3$ & $99.39 \pm 0.05$ \\
\hline
\end{tabular}

Table A.3: N-1 efficiencies for CMUP muon identification variables[58]

\begin{tabular}{|c|c|c|}
\hline \hline & CMX & \\
Variable & Data & $Z^{0} \rightarrow \mu^{+} \mu^{-} \mathrm{MC}$ \\
\hline$d_{0}$ & $99.2 \pm 0.2$ & $99.74 \pm 0.04$ \\
$E_{\text {em }}$ & $97.6 \pm 0.4$ & $96.28 \pm 0.16$ \\
$E_{\text {had }}$ & $98.0 \pm 0.3$ & $96.46 \pm 0.16$ \\
$N_{A x}$ SL, $N_{S t}$ SL & $97.1 \pm 0.4$ & $97.95 \pm 0.12$ \\
\hline iso & $97.6 \pm 0.4$ & $97.71 \pm 0.13$ \\
\hline CMX stub* $^{*}$ & $99.06 \pm 0.28$ & $99.86 \pm 0.04$ \\
$\left|\Delta x_{C M X}\right|$ & $99.5 \pm 0.2$ & $99.86 \pm 0.03$ \\
\hline
\end{tabular}

Table A.4: N-1 efficiencies for CMX muon identification variables[58]. The CMX stub efficiency assumes the $\rho$ cut has already been made. 


\section{A.2 Additional Fake Rate Studies}

\section{A.2.1 Isolation $\mathrm{v} \cdot \chi^{2}$ independence}

All photon selection cuts in table 6.1 were applied to select fake photon candidates in HERWIG[79] dijet MC simulation. Truth information was used to ensure that prompt photons were not considered (see section 7 ). The CES $\chi^{2}$ distribution is plotted in figure A.1 for the candidates which pass and fail the calorimeter isolation cut separately. The two distributions are in good agreement. The CES $\chi^{2}$ and Iso4 quantities are concluded to be independent of each other for fake photon candidates.

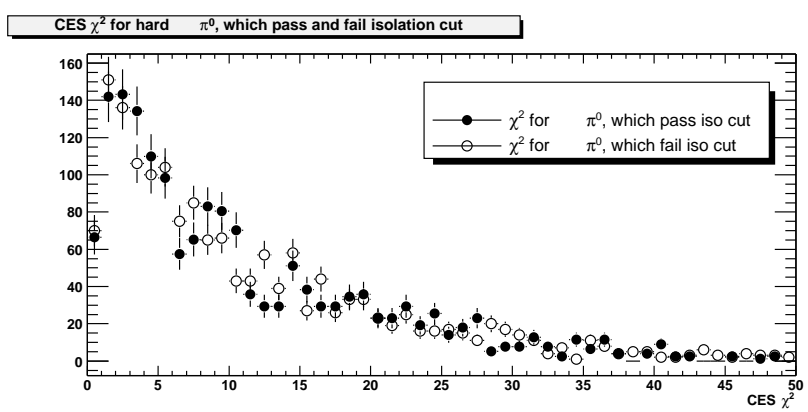

Figure A.1: CES $\chi^{2}$ distribution for fake photon candidates in HERWIG dijet MC simulation. Candidates which pass and fail the Iso4 cut are plotted separately.

\section{A.2.2 Boundary choice for CES method}

The boundary choice for the $\chi^{2}$ v. Iso method in section 7.4.1 is varied in order to see how dependent the resulting prompt $\gamma$ fraction is on the choice of regions A,B,C and D. The results are shown in table A.5. No systematic dependence on the boundaries is observed.

\section{A.3 Other Studies}

\section{A.3.1 Dependence on $\eta$ and $\phi$}

Figure A.2 shows the corrected fake rate integrated over $7<E_{T}^{\text {Jet }}<40$ as a function of $\eta$ and $\phi$. Whilst the fake rate is independent of $\phi$ a significant 


\begin{tabular}{|l|r|r|r|}
\hline $\begin{array}{l}\text { Lower Iso boundary } \\
\text { of regions B and D }\end{array}$ & Iso4 $/ E_{t}<0.1$ & Iso4 $/ E t<0.15$ & Iso $/ E t<0.2$ \\
\hline $7-9(\mathrm{GeV})$ & $0.84 \pm 0.05$ & $0.83 \pm 0.05$ & $0.85 \pm 0.05$ \\
$9-13(\mathrm{GeV})$ & $0.63 \pm 0.04$ & $0.64 \pm 0.05$ & $0.60 \pm 0.05$ \\
$13-20(\mathrm{GeV})$ & $0.57 \pm 0.05$ & $0.56 \pm 0.05$ & $0.54 \pm 0.05$ \\
\hline $\begin{array}{l}\text { Lower Iso boundary } \\
\text { of regions B and D }\end{array}$ & IsoC $<0.02$ & IsoC $<0.04$ & IsoC $<0.06$ \\
\hline $20-40(\mathrm{GeV})$ & $0.27 \pm 0.05$ & $0.28 \pm 0.05$ & $0.27 \pm 0.05$ \\
\hline
\end{tabular}

Table A.5: The variance of $F_{Q C D}$ on the choice of boundaries for method 7.4.1: IsoC $=($ Iso4-2) $/(20-\mathrm{Et})$

dependence on $\eta$ due to the CES fiduciality requirements imposed on the photon candidates is observed. Any effect due to this $\eta^{\text {jet}}$-dependence of the fake rate is implicitely taken into account if one makes the reasonable assumption that the $\eta^{\text {jet }}$ distribution is the same in $W+$ jet and dijet events.
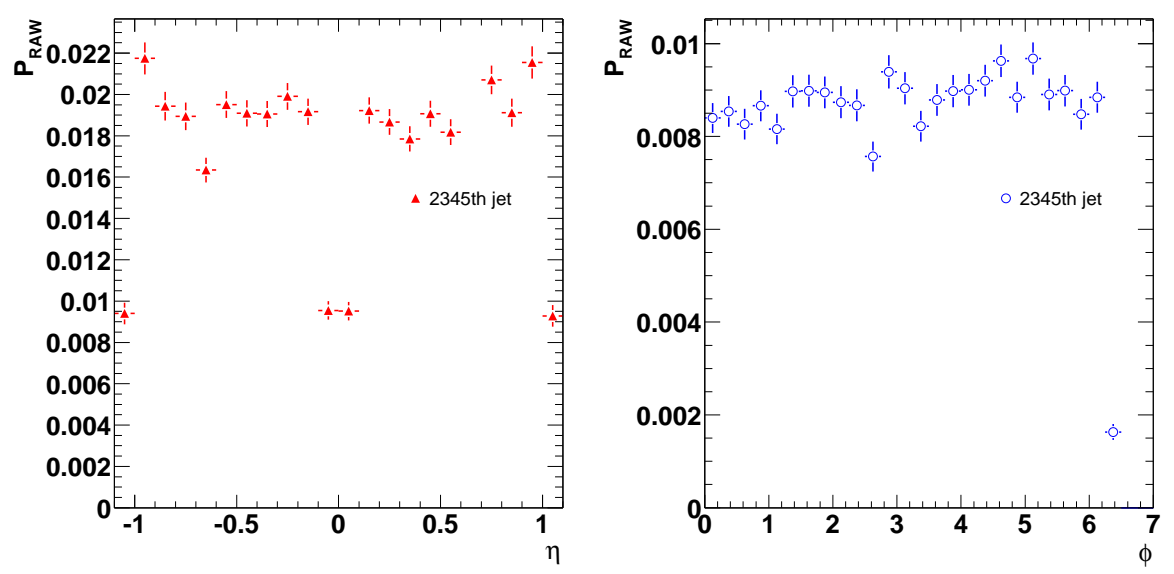

Figure A.2: The uncorrected fake rate as a function of $\eta$ and $\phi$ 


\section{Bibliography}

[1] D. Acosta et al., "Measurement of W Gamma and Z Gamma Production in $\mathrm{P}$ anti-P Collisions at $\mathrm{s}^{* *}(1 / 2)=1.96-\mathrm{TeV}$ ". FERMILAB-PUB-04246-E (Oct 2004) [HEP-EX 0410008]

[2] S. Eidelman et al., Phys. Lett. B 592, 1 (2004)

[3] S. L. Glashow, Nucl. Phys., 22, 579 (1961)

[4] S. Weinberg, Phys. Rev, Lett., 19, 1264 (1967)

[5] A. Salam, Proceedings of the Eight Nobel Symposium, ed. by N. Svartholm, Alqvist \& Wiskell, Stockholm, (1968).

[6] LEP EWWG, "A Combination of Preliminary Results on Gauge Boson Couplings Measured by the LEP Experiments", LEPEWWG/TGC/200202 (see also http://lepewwg.web.cern.ch/LEPEWWG/lepww/tgc/

[7] P. W. Higgs. "Broken Symmetries, Massless Particles and Gauge Fields", Phys. Lett., 12 (1964) 132-133

[8] P. W. Higgs. "Spontaneous Symmetry Breakdown without Massless Bosons", Phys. Rev., 145 (1966) 1156-1163

[9] P. W. Higgs. "Broken Symmetries and the MAsses of Gauge Bosons", Phys.Lett., 13 (1964) 508-509

[10] K. Hagiwara et al., "Probing the Weak Boson Sector in $e^{+} e^{-} \rightarrow$ $W^{+} W^{-"}$, Nucl. Phys. B282, 253 (1987)

[11] J. Ohnemus, U. Baur, "Order- $\alpha$-s calculations of hadronic $W^{ \pm} \gamma$ and $Z \gamma$ production", Phys. Rev. D47 (1993) 940;

[12] D. Benjamin, A. Goshaw, M. Kirby, B. Heinemann, H. Hayward, N. Tanimoto, "Theoretical Predictions of $W \gamma$ and $Z \gamma$ Production in Run II", CDF note 6619. 
[13] James Stirling, personal communication.

[14] Uli Baur, personal communication.

[15] U. Baur, S.Errede, J.Ohnemus,FSU-HEP-930322, UIUC-HEP-93-01, DTP/93/14 (March 1993)

[16] B. Abbott et al., " $\mathrm{Z} \gamma$ Production in Anti-p p Collisions s** $(1 / 2)$ $=1.8-\mathrm{TeV}$ and Limits on Anomalous Z Z $\gamma$ and Z $\gamma \gamma$ Couplings." Phys.Rev.D57:3817-3821,1998. [HEP-EX 9710031]

[17] G.P. Lepage, "VEGAS: An Adaptive Multi-dimensional Integration Program", Cornell preprint CLNS 80-447, March 1980

[18] A. Pukhov et al., "CompHEP - a package for evaluation of Feynman diagrams and integration over multi-particle phase space.", hep-ph/9908288.

[19] F. Maltoni and T. Stelzer, hep-ph/0208156.

[20] Landsberg, G., "Test of the Standard Model of Electroweak interactions by Measureing the Anomalous $Z Z \gamma$ and $Z \gamma \gamma$ Couplings", Thesis, State University of New York at Stony Brook. (1994)

[21] U. Baur, E. L. Berger, "Probing the $W W \gamma$ vertex at the Fermilab Tevatron Collider", Phys. Rev. D 41, 1476 (1990).

[22] U. Baur, T. Han and J. Ohnemus, "QCD corrections and Anomalous Couplings in $Z \gamma$ Production at Hadron Colliders", Phys. Rev. D 57, 2823 (1998).

[23] H.L. Lai et al., "Global QCD Analysis of Parton Structure of the Nucleon: CTEQ5 Parton Distributions", Eur.Phys.J.C12:375-392,2000. [HEP-PH 9903282]

[24] A. D. Martin, R. G. Roberts, W. J. Stirling, R. S. Thorne, "Uncertainties of predictions from parton distributions I: Experimental errors", Eur.Phys. J. C23, 73 (2002)

[25] Fermilab's Chain of Accelerators, Accelerator Details: the Proton Source http://www-bd.fnal.gov/public/proton.html

[26] Fermilab Main Injector Technical Design Handbook http://www-fmi.fnal.gov/fmiinternal/MI_Technical_Design/index.html

[27] Fermilab's Chain of Accelerators, Accelerator Details: the Antiproton Source http://www-ad.fnal.gov/public/antiproton.html 
[28] The Antiproton Source Rookie Book http://wwwbdnew.fnal.gov/pbar/documents/PBAR_Rookie_Book.PDF

[29] Michelangelo L. Mangano, hep-ph/9711337 (1997)

[30] CDF Collaboration, The CDF II Detector: Technical Design Report, Fermilab-PUB-96/390-E (1996)

[31] T.K. Nelson, "The CDF Layer 00 Detector", CDF note 6251.

[32] L. Miller, "Nuclear Instruments and Methods in Physics Research A", $518,1(2004)$

[33] T.K. Nelson, "Nuclear Instruments and Methods in Physics Research A" 485, 1 (2002)

[34] B. Ashmanskas et al., "Open-cell Chamber to Replace the CTC", CDF note 3648

[35] D. Ambrose, "The Central Outer Tracker for Run II at CDF", Nuclear Instruments and Methods in Physics Research Section A, Vol 518, Issues 1-2, p2 42-44.

[36] C. Hays et al., "The COT Pattern Recognition Algorithm and Offline Code", CDF note 6992.

[37] A Time-of-Flight System for CDF

http://www-cdf.fnal.gov/upgrades/TOF/doc/notes/cdf2573/toffinal.html

[38] CDF Run II Tracking Software: Outside-In Tracking Description http://www-cdf.fnal.gov/upgrades/computing/projects/reconstruction/ tracking/user-docs/OIoverview.html

[39] Byeong Rok Ko, Soeg H. Oh, Chiho Wang, "SVXII Stand-alone Tracking" CDF note 6440.

[40] D. Glenzinski, M. Herndon, C.-J. Lin, J. Thom, A. Yagil, "Determination of the Run IIa COT Tracking Efficiency Using the W-No-Track Sample", CDF note 5973.

[41] T. Nelson, "Silicon Tracking for Plug Electrons", CDF note 5970

[42] T. Nelson, "Forward Electron Tracking with PhoenixMods Package", CDF note 6278 
[43] L. Balka et al., "Nuclear Instruments and Methods in Physics Research A", 267, 1 (1988)

[44] A. Solodsky, The CDF Collaboration, "The Shower-Maximum Detector for CDF II", FERMILAB-CONF-98/085-E (1990)

[45] Partos, D., "Measurement of the $\sqrt{s}$ Dependence of Isolated Direct Photon Production in Proton-Antiproton Collisions", Thesis, Brandeis University, Physics Department.

[46] CDF Run 2 Muon Chambers, http://www-cdfonline.fnal.gov/ cdf_muon/chambers.html

[47] T. Dorigo, "Nuclear Instruments and Methods in Physics Research A", 461, 1 (2001)

[48] S. Klimenko, J. Konigsberg, T.M. Liss, "Averaging of the inelastic crosssections measured by the CDF and E811 experiments", Fermilab-FN0741

[49] D. Acosta, "The CDF Cherenkov luminosity monitor", Nuclear Instruments and Methods in Physics Research Section A, Vol 461, Issues 1-3, p540-544, (2001)

[50] E811 Collaboration, "A Measurement of the Proton Anti-Proton Total Cross Section at S**(1/2) =1.8 TeV", Phys.Lett.B445:419-422,1999

[51] CDF Run2 Triggers and Filters Report Group Triggers and Bits on Replicated Online Production Report - physics table PHYSICS_1_03_v-2 http://www-cdf.fnal.gov/internal/upgrades/daq_trig/twg/TriggerTables /Reports/PHYSICS_1_03_v2.html

[52] E.J. Thomson et al., IEEE Transactions on Nuclear Science, Vol. 49, No. 3, June 20021

[53] http://www-cdf.fnal.gov/internal/physics/ewk/tools_and_datasets/goo d_run_list.html

[54] T.Affolder et al., "Measurement of $d$ sigma/dy for high mass Drell-Yan $e^{+} e^{-}$pairs from pp-bar collisions at sqrt(s) $=1.8 \mathrm{TeV}$.", Phys. Rev. D 63, 011101(R) (2001).

[55] H. Ray et al., "Level 2 Electron Trigger Efficiencies for Run II", CDF note 6098 
[56] R. Wagner, "Electron Identification for Run II: Understanding and Using Lshr". CDF note 6249

[57] Y.-K. Kim, J. Nielsen, L. Tompkins, G. Veramendi, "Trigger Efficiencies for High $P_{T}$ Electrons", CDF note 6234

[58] L. Cerrito, V. Martin, "Muon Cuts and Efficiencies for 4.11 Analyses", CDF note 6825

[59] A. Hocker and W. Sakumoto," Event $|Z v t x|<60 \mathrm{~cm}$ Cut Efficiency for Run II", CDF note 6660

[60] R. G. Wagner, "Electron Identification for Run II: Algorithms", CDF note 5456

[61] M. Coca and E. Halkiadakies, "Central Electron Identification Efficiencies for the $200 p b^{-1}$ Run 2 Dataset", CDF note 6580

[62] D. Amidei et al., "Measurement of $W \rightarrow \mu \nu$ and $Z \rightarrow \mu \mu$ production cross-sections and $R$ using CDF Run 2 Data", CDF note 6711

[63] D. Amidei et al., "Measurement of $\sigma \times B(W \rightarrow e \nu), \sigma \times B(Z \rightarrow e e)$ and the ratio $R$ using CDF Run 2 Data", CDF note 6681

[64] C. Issever et al., "Plug Electron Baseline Cuts and Efficiencies for Summer 2003", CDF note 6789

[65] D. Goldstein et al., "A measurement of the $t \bar{t}$ cross-section using dileptons in the central and endplug detectors", CDF note 6588

[66] Mge Karagz nel, "Searches for New Physics Using High Mass Dimuons at the CDF II Experiment", CDF not 7296.

[67] Victoria Martin, personal communication.

[68] Aidan Robson, Oxford University, personal communication.

[69] D. Acosta et al., "First Measurements of Inclusive W and Z Cross Sections From Run II of the Tevatron Collider", FERMILAB-PUB-04-159-E (Jun 2004), [HEP-EX 0406078]

[70] Guilia Manca and Young-Kee Kim, " $Z^{0}$ Cross-Section Measurement Using Run II electrons", CDF note 6281

[71] W. K. Sakumoto et al., "W/Z Cross Section Predictions for $\sqrt{s}=1.96$ TeV", CDF note 6341 
[72] P. Wilson, "Calorimeter Isolation and Lateral Shower Leakage for Photons and Electrons", CDF Note 4170 (1998)

[73] J.-F. Arguin. B. Heinemann. A. Yagil, "The z-Vertex Algorithm in Run II", CDF Note 6238 (2002)

[74] D. Benjamin, A. Goshaw, M. Kirby, B. Heinemann, H. Hayward "Underlying Event Studies and Photon ID Efficiencies in Run II", CDF Note 6608 (2003)

[75] N. Tanimoto "Photon ID Efficiencies for $W+\gamma$ analysis in Run II", CDF Note 6857 (2004)

[76] F. Abe et al., The CDF Collaboration, "Prompt photon cross section measurement in p anti-p collisions at $\sqrt{s}=1.8$ TeV", Phys. Rev. D 48, 2998 (1993);

[77] Y. Liu, http://www-cdf.fnal.gov/internal/people/links/YanwenLiu/cescpr.html

[78] M. Kim, R. Culbertson, R. Blair, "CPR Material Counts of CDF Run II", CDF Note 6101.

[79] G. Corcella, I.G. Knowles, G. Marchesini, S. Moretti, K. Odagiri, P. Richardson, M.H. Seymour and B.R. Webber, HERWIG 6.5 ,JHEP 0101 (2001) 010 [hep-ph/0011363]; hep-ph/0210213.

[80] M.L. Mangano, M. Moretti, F. Piccinini, R. Pittau, A. Polosa, ALPGEN, a generator for hard multiparton processes in hadronic collisions, JHEP 0307:001,2003, hep-ph/0206293.

[81] T. Sjstrand, P. Edn, C. Friberg, L. Lnnblad, G. Miu, S. Mrenna and E. Norrbin, PYTHIA 6.154: Computer Phys. Commun. 135 (2001) 238 (LU TP 00-30, hep-ph/0010017)

[82] D. Acosta et al., "The CLC Minimum Bias Trigger", cdf note 5861.

[83] F. Abe et al. (CDF Collaboration), "Phys. Rev. Lett. 74, 2626 (1995)

[84] S. Abachi et al. (D0 Collaboration), "Phys. Rev. Lett. 74, 2632 (1995)

[85] P. Azzi-Bacchetta et al., "Proposal for a Very Low Mass, Very Small Radius Silicon Layer In the CDF II Upgrade, CDF Note 4924 (1999)

[86] Kevin T. Pitts, "Nuclear Physics B - Proceedings Supplements 61, 230 (1998) 
[87] Ch. Paus et al., "Nuclear Instruments and Methods in Physics Research A 461, 579 (2001)

[88] Ryutaro Oishi et al., "Nuclear Instruments and Methods in Physics Research A 453, 227 (2000)

[89] G. Ascoli et al., "CDF Central Muon Detector, Fermilab-Pub-87/179-E (1987)

[90] Tommaso Dorigo, "Nuclear Instruments and Methods in Physics Research A 461, 560 (2001)

[91] Y. Gotra et al., "Run II Muon Trigger Efficiency Measurement, CDF Note 6162 (2003)

[92] A. Taffard, "Run II Cosmic Ray Tagger, cdf note 6100.

[93] http://www-cdf.fnal.gov/internal/physics/photon/docs/cuts.html

[94] D. Benjamin, "Determination of the QCD Jet $\rightarrow$ Fake Photon Background in Run I $V+\gamma$ Data Samples., cdf note 5439.

[95] A. Korytov, A. Pronko, A. Safonov, "Measurement of the Ratio of Charged Particle Mulitplicities in Gluon and Quark Jets.", cdf note 5899

[96] P. Wilson, "Calorimeter Isolation and Lateral Shower Leakage for Electrons and Photons",CDF note 4170

[97] B. Heinemann, A.C. Wyatt, "Correction for Leakage Energy in the Central and Plug Calorimeters in Run II", CDF note 6167

[98] D. Acosta et al., The CDF Collaboration, "Search for New Physics in Photon-Lepton Events in p anti-p Collisions at $\mathrm{s}^{* *}(1 / 2)=1.8 \mathrm{TeV}$ ", Phys. Rev. Lett. 89, 041802 (2002).

[99] H. K. Gerberich, "Photon fake rate using the CPR", CDF Note 6838.

[100] B. Abbott et al., "Studies of WW and WZ Production and Limits on Anomalous WW $\gamma$ and WWZ Couplings", Phys. Rev. D 60, 072002 (1999).

[101] D. Benjamin et al., "Event Selection in CDF Run I $V+\gamma$ Analysis", CDF note 5999

[102] D. Benjamin et al.," Cross Section Measurements for W+photon and Z+photon Production in Run II", CDF note 6366 
[103] U. Baur and E.L. Berger, "Probing the $W W \gamma$ vertex at the Fermilab Tevatron Collider", Phys. Rev. D 41, 1476 (1990).

[104] D. Benjamin, A. Goshaw, M. Kirby, N. Tanimoto, "A comparison of $W+\gamma$ MC generators", CDF note to come

[105] M. Coca et al., "W $\rightarrow e \nu$ Cross Section Analysis with Run II Data", CDF note 6300

[106] K. Bloom et al., "Updated Measurements of $\sigma(p \bar{p} \rightarrow W \rightarrow \mu \nu), \sigma(p \bar{p} \rightarrow$ $Z \rightarrow \mu \mu$ ), and $R$ using CDF Run II Data", CDF note 6302

[107] Y.-K. Kim, G. Manca, " $Z^{0}$ to electrons Cross Section measurement with Run II data", CDF note 6281

[108] http://www-cdf.fnal.gov/internal/physics/top/r2leptons/etf/etfnewh ome/BaseLineCuts/baselinecuts_12jun03.html

[109] http://www-cdf.fnal.gov/internal/physics/photon/docs/cuts.html

[110] E. Halkiadakis, talk in Joint Physics Meeting 07/25/2003

[111] V. Martin, talk in Joint Physics Meeting 07/25/2003

[112] A. Robson et al., "A Measurement of $\sigma \cdot \operatorname{Br}\left(Z^{0} \rightarrow e^{+} e^{-}\right)$using Run 2 Central and Plug Electrons in 72 pb $^{-1}$ )", CDF note 6642

[113] H. Hayward and B. Heinemann, "Determination of the QCD Jet Background to Isolated Photons ", CDF note 6363

[114] M. Kirby, N. Tanimoto, et al. , "Photon ID Efficiencies in Run II", CDF note 6608

[115] S. Chuang et al., "Estimate of the amount of material in the CDF tracker using high-Pt electrons", CDF note 6573

[116] Moliere Radius, http://rd11.web.cern.ch/RD11/rkb/PH14pp/node115 .html

[117] Kirby, M., "Measurement of $W+\gamma$ Production in Proton-Antiproton Collisions at $\sqrt{s}=1.96$ TeV", Thesis, Department of Physics, Duke University. (2004)

[118] Tanimoto, N., "A Measurement of the $W+\gamma$ Production Cross-section in the Muon Decay Channel in $p \bar{p}$ Collisions at $\sqrt{s}=1.96 \mathrm{TeV}$ ", Thesis, Okayama University, Japan (2004) 